\title{
Investigation of the Relative Abundance of Heavy versus Light Nuclei in Primary Cosmic Rays using Underground Muon Bundles
}

\author{
A dissertation \\ submitted by \\ Nakamuthu Sundaralingam
}

In partial fulfillment of the requirements

for the degree of

Doctor of Philosophy

in

Physics

TUFTS UNIVERSTY

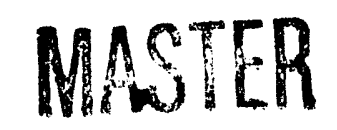

DISTRIBUTION OF THIS DOCUMENT IS UNLIMITED

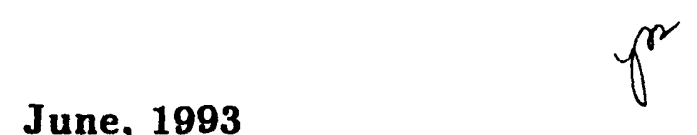




\title{
ABSTRACT OF THE DISSERTATION
}

\section{Investigation of the Relative Abundance of Heavy versus Light Nuclei in Primary Cosmic Rays using Underground Muon Bundles}

\author{
by Nakamuthu Sundaralingam, Ph.D. \\ Dissertation Director: Prof. W. A. Mann
}

We study multiple muon events (muon bundles) recorded undergrcund at a depth of $2090 \mathrm{mwe}$. To penetrate to this depth, the muons must have energies above 0.8 $\mathrm{TeV}$ at the Earth's surface; the primary cosmic ray nuclei which give rise to the observed muon bundles have energies at incidence upon the upper atmosphere of 10 to $10^{5} \mathrm{TeV}$. The events are detected using the Soudan 2 experiment's fine grained tracking calorimeter which is surrounded by a $14 \mathrm{~m} \times 10 \mathrm{~m} \times 31 \mathrm{~m}$ proportional tube array (the "active shield"). Muon bundles which have at least one muon traversing the calorimeter, are reconstructed using tracks in the calorimeter together with hit patterns in the proportional tube shield. All ionization pulses are required to be coincident within 3 microseconds.

A goal of this study is to investigate the relative nuclear abundances in the primary cosmic radiation around the "knee" region $\left(10^{3}-10^{4} \mathrm{TeV}\right)$ of the incident 
energy spectrum. Four models for the nuclear composition of cosmic rays are considered: The Linsley model, the Constant Mass Composition model (CMC), the Maryland model and the Proton-poor model [Forti et al., Phys. Rev. D42, 3668 (1990)]. A Monte Carlo which incorporates one model at a time is used to simulate events which are then reconstructed using the same computer algorithms that are used for the data. Identical cuts and selections are applied to the data and to the simulated events.

A systematic search for possible correlations involving muon bundle multiplicity, intermuon separation within bundles, and bundle zenith angle has been carried out in this work; comparable analyses from underground experiments have seldom appeared in the literature. Within a zenith angle range from $15^{\circ}$ to $60^{\circ}$, we observe the average muon multiplicity to remain constant, independent of zenith. The average intermuon separation within a muon bundle $\left\langle R_{i j}\right\rangle$, as measured in the plane transverse to the bundle direction, is observed to increase mildly with increasing zenith angle. Additionally, there is an apparent increase of $\left\langle R_{i j}\right\rangle$ with increasing muon bundle multiplicity, for $N_{\mu}>8$ muons. Neither of these mild correlations are included in the commonly used parametrizations of the nuclear composition models.

It is the lighter composition models, namely the CMC model and the Linsley model, that show better overall agreement with our data, especially in the muon bundle multiplicity distribution and in the variation of average multiplicity with zenith angle. Independent fits to bundle distributions involving $N_{\mu},\left\langle N_{\mu}\right\rangle$ versus cosine of zenith angle and $\left\langle N_{\mu}\right\rangle$ versus $\left\langle R_{i j}\right\rangle$ were carried out using a simplified two-component nuclear composition. From the rough agreement among these fits, we infer that the primary cosmic radiation consists predominantly (74 $\pm 15 \%)$ of protons and relatively light nuclei ( $\mathrm{He}, \mathrm{C}, \mathrm{N}, \mathrm{O}$ ); elements heavier than $\mathrm{Mg}$ form a smaller fraction $(26 \pm 15 \%)$. 


\section{Acknowledgements}

I would like to thank all of those within Tufts University Community who, since I first arrived, in some way affected my life so profoundly, intellectually and socially.

First and foremost, I would be forever grateful to my adviser, Professor W. A. Mann. His ability and willingness to share his knowledge, insight and experience in physics and other related fields are surpassed only by his infinite patience. Without his direction, this thesis dissertation could never have reached completion. Not only was his advice regarding work on the Soudan 2 group always welcome, but also his broader perspectives on life exemplified by his generosity and kindness always will be remembered.

Among others within the Tufts Department of Physics and Astronomy, who deserve special thanks is Professor W. Oliver. I had never known as much about electronics until I worked under his waishful tutelage on the veto shield pre-amplifier board. By communicating his experience in electronics and physics, he helped me to develop my own knowledge in these fields. As a result, I was able to discover for myself the mysteries and joys of studying cosmic ray physics. Special thanks also to Professor T. Kafk? for his generous assistance in writing the enormous amount of computer code without which my analysis would have been impossible. His thorough review of my thesis dissertation was also greatly appreciated.

The Tufts-Soudan group meetings were always enhanced when graced with the presence of Prof. A. Napier, Prof. R. Milburn, and Prof. J. Schneps. These outsianding professors sacrificed time from their own busy scuedules to share their thoughts with ine.

I would also like to thank my many colleagues who made my stay at Tufts 
University so pleasurable. Particularly, I am indebted to Kanthy and Dharmaratna whose help and moral support when I first arrived in the USA will never be forgotten. Sincere thanks are also due to Stephane Willocq who gave me much fruitful advice especially with computers. I must thank Larry McMaster who, besides running the veto shield shop, provided much-needed technical support for the shield deployment.

While working at the Soudan underground mine, I was fortunate to be helped by professionals who were always pleasant and cheerful. My special thanks to Jim Beaty who helped me in a number of ways. Also thanks to my fellow graduate students at University of Minnesota, especially Sue Schubert for her kind help in developing the Monte Carlo simulation program.

Finally, this thesis which represents the culmination of my life long study of science could never have been possible without my wife Vimala's encouragement and constant support. Her patience in enduring the spartan lifestyle of a struggling graduate student reflects of deeper bond of love and respect that all have for each other. These acknowledgments could not be complete without thanking my daughters, Shankeertha and Swetha, both of whom have inspired me with greater confidence in a happy future. 
This dissertation is dedicated to my parents for their love and support. 


\title{
Table of Contents
}

\begin{abstract}
..................... is
Acknowledgements $\ldots \ldots \ldots \ldots \ldots$ iv
\end{abstract}

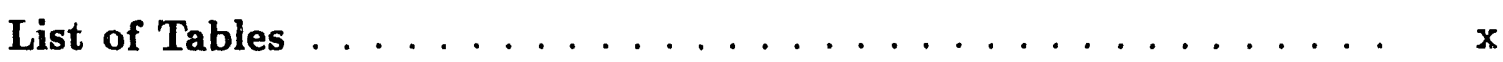

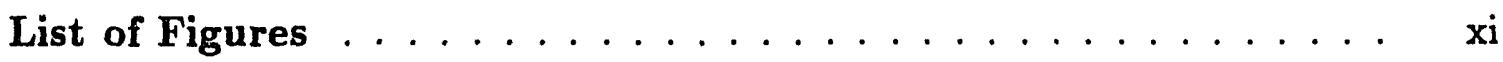

1. Cosmic Rays ........................ 1

1.1. Origin of Cosmic Rays and Their Composition . . . . . . . . . . 2

1.2. Study of Cosmic Rays: A Historical Perspective . . . . . . . . . . 4

1.3. Solar and Cosmic Ray Relative Abundances . . . . . . . . . . . . . 5

1.4. Acceleration Mechanisms . . . . . . . . . . . . . . 7

1.5. Direct and Indirect Methods for the Study of Primary Cosmic Rays . 9

1.5.1. Direct Methods .................. 10

1.5.2. Indirect Methods: Secondary Cosmic Rays . . . . . . . . . . . 11

1.6. Detectors to Study Secondary Cosmic Rays . . . . . . . . . . . . 12

1.6.1. Surface Arrays . . . . . . . . . . . . . . . 13

1.6.2. Underground Detectors . . . . . . . . . . . . . . . 14

2. Soudan 2 Nucleon Decay Detector . . . . . . . . . . . . 16

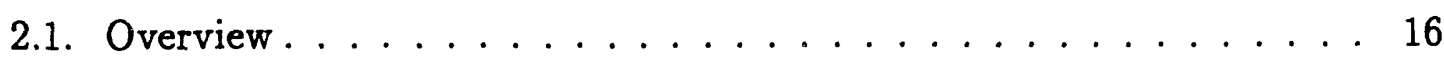

2.2. The Central Detector . . . . . . . . . . . . . . 18

2.3. The Active Shield . . . . . . . . . . . . . . . . . . 21

2.4. Electronics for the Veto Shield . . . . . . . . . . . . . . . . . 23 
2.4.1. The Digital Output Card . . . . . . . . . . . 23

2.4.2. Digital Readout Module . . . . . . . . . . . . 24

2.5. The HPW Enhancement Array . . . . . . . . . . . . 25

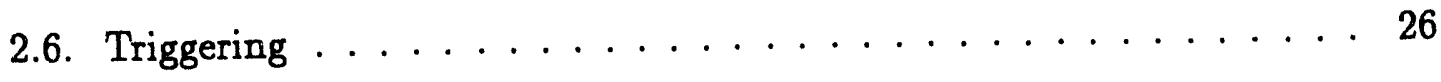

2.7. Software and Data Processing . . . . . . . . . . . . 28

2.8. The Event Time; SEARCH Output . . . . . . . . . . . . 30

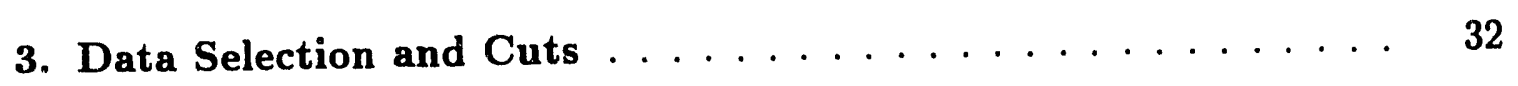

3.1. Shield Hit Configurations . . . . . . . . . . . . . . 32

3.2. Data Processing and Initial Event Selection . . . . . . . . . . 35

3.3. Selection of Muon Tracks in the Central Detector . . . . . . . . 36

3.4. Reconstruction of Muon Tracks Using the Shield . . . . . . . . . . . 42

3.5. Reconstructed Muon Bundles ... . . . . . . . . . . . 48

3.6. Displays of Multiple Muon Events . . . . . . . . . . . . . 53

4. Detector Acceptance, Efficiencies and Uncertainties . . . . . . 61

4.1. Acceptance of the Central Detector . . . . . . . . . . . 61

4.2. Efficiency of the Active Shield . . . . . . . . . . . . . 61

4.3. Zenith Angle and Azimuthal Angle Distributions . . . . . . . 66

4.4. Error Analysis . . . . . . . . . . . . . . . . 72

4.4.1. Errors on $R_{i j}$ for Central Detector Muons . . . . . . . . 72

4.4.2. Errors on $R_{i j}$ for Shield $M_{\perp}$ and $M_{\|}$Muons . . . . . . . 72

5. Simulation of Underground Muon Bundles . . . . . . . . . 75

5.1. Overview of the Monte Carlo Simulation . . . . . . . . . . . 75

5.1.1. Primary Cosmic Ray Energy Spectrum . . . . . . . . . . . 77

5.1.2. Muon Energy Losses in the Rock . . . . . . . . . . . . . 78

5.1.3. Muon Bundle Multiplicity . . . . . . . . . . . . . 79

5.1.4. Separation of Muons from the Bundle Core . . . . . . . . 81 
5.2. Models of Cosmic Ray Composition . . . . . . . . . . . . . . . . 82

5.3. Uncertainties in the Monte Carlo Simulation . . . . . . . . . . . . 87

5.3.1. Earth overburden above the detector . . . . . . . . . 87

5.3.2. Detector geometry and geometric efficiency . . . . . . . . 88

5.3.3. Hadronic interaction model for cosmic rays . . . . . . . . . 88

6. Properties of Multiple Muon Events At Soudan . . . . . . . . . 92

6.1. Additional Cuts to the Data . . . . . . . . . . . . . . . 92

6.2. Track Categories by Reconstruction . . . . . . . . . . . . . 95

6.3. Muon Bundle Multiplicity Distribution . . . . . . . . . . . . . . . 98

6.4. Absolute Rate for Multiple Muon Events . . . . . . . . . . . . . . 104

6.5. Muon Separation within Bundles . . . . . . . . . . . . 111

7. Search for Correlations in Properties of Muon Bundles . . . . . . 113

7.1. Average Multiplicity versus Zenith Angle . . . . . . . . . . . . . 113

7.2. Intermuon Separation versus Zenith Angle . . . . . . . . . . . . 116

7.3. Intermuon Separation versus Multiplicity . . . . . . . . . . . 120

7.4. Protons versus Iron in Primary Cosmic Radiation . . . . . . . . . . . 124

8. Discussion and Conclusion . . . . . . . . . . . . . . . 127

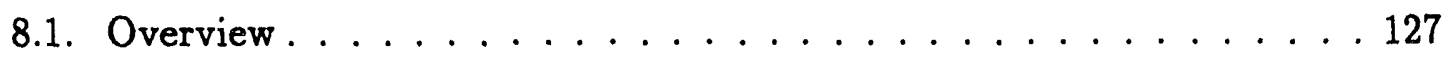

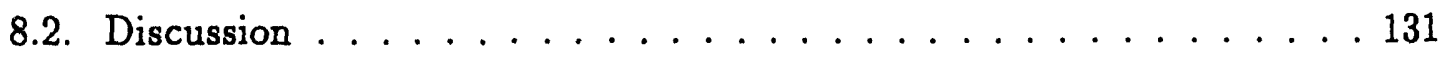

8.3. Conclusion . . . . . . . . . . . . . . . . . . 132

Appendix A. The Soudan 2 Collaboration . . . . . . . . . . 134

Appendix B. Data Summary Tape Format . . . . . . . . . . . 136

Appendix C. Error Calculation . . . . . . . . . . . . . 143 
Appendix D. Translator Circuits for the HPW Slectronics $\ldots \ldots 146$

๖.1. HPW Chambers . . . . . . . . . . . . . . . . . . . 146

D.2. Translator Circuits and Conversion Boards . . . . . . . . 147

Relerences . . . . . . . . . . . . . . . . . 150 


\section{List of Tables}

3.1. Run Statistics for the 1990 and 1991 data samples . . . . . . . . . . 38

3.2. Muon classification for tracks within a muon bundle. . . . . . . . . . 49

4.1. Veto shield area as of June 1991. . . . . . . . . . . . . . . . 62

4.2. Number of veto shield hits associated with each central detector muon track, from 376 muon events scanned by physicists. . . . . . . 63

4.3. Run statistics for 54,035 events with two or more candidate muons from the 1991 data, used to calculate efficiency of the shield. . . 64

5.1. Normalization factors $K, K^{\prime}$, slopes $\gamma$, before and after cutoff energy

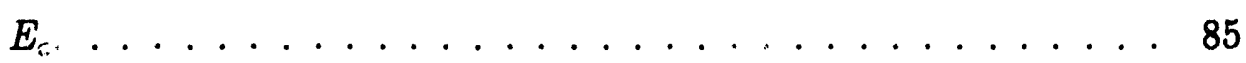

6.1. Multiple muon events in the 1991 data. . . . . . . . . . . . . . . 96

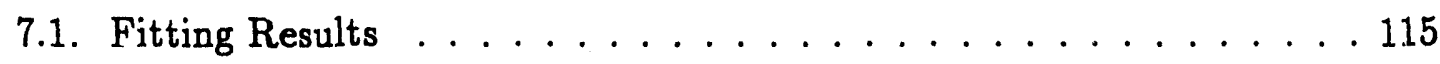

7.2. Percentages of protons and iron in the primary cosmic rays for rock density of $2.80 \mathrm{~g} \mathrm{~cm}^{-3} \ldots \ldots \ldots \ldots \ldots$ 


\section{List of Figures}

1.1. The All Particle Energy Spectrum . . . . . . . . . . . . . . 3

1.2. Solar and Cosmic Ray abundances. Solid circles and open circles show cosmic rays for low (up to $0.3 \mathrm{GeV}$ ) energy and high (up to 2 $\mathrm{GeV}$ ) energy repectively; open diamonds show solar abundances; all are relative to the abundance of silicon. . . . . . . . . . 5

1.3. The development of secondary cosmic rays and their detection. . . . . 12

2.1. Plane view of the Soudan 2 detector. . . . . . . . . . . . . . 17

2.2. A view of a tracking calorimeter module. . . . . . . . . . . . . . 19

2.3. Cut away longitudinal view of a drift tube. . . . . . . . . . . . . 20

2.4. A module Wire Plane of the Soudan tracking calorimeter. . . . . . . . 20

2.5. End view of three adjacent veto shield manifolds. . . . . . . . . . 22

2.6. Schematic diagram of a digital output card. . . . . . . . . . . 24

2.7. A through-going single muon track with a small knock-on electron , 27

3.1. A single hit and Coincident-Two-Layer hits. . . . . . . . . . . 33

3.2. Adjacent Groups. . . . . . . . . . . . . . . . . . 33

3.3. Multiple muon events per day. . . . . . . . . . . . . . . . 37

3.4. Distributions for the time of occurence of muon events as estimated using (a) $T_{C D}$ and (b) $T_{V S} \ldots \ldots \ldots \ldots$

3.5. The distribution of the time difference between calculated time slot (CALTS) and shield time slot (TSHLD) $\left(T_{C D}-T_{V S}\right) \ldots \ldots 39$ 
3.6. Coordinate system for the Soudan-2 cavern. Here $\hat{u}$ is the unit vector along a muon direction. $\mathrm{X}$ and $\mathrm{Z}$ axes are horizontal and the $\mathrm{Y}$ axis is vertical. $\alpha$ is the angle between $\mathrm{X}$ axis and $\hat{u}, \beta$ is the angle between $Y$ axis and $\hat{u}$ (zenith angle) and $\gamma$ is the angle between $\mathrm{Z}$ axis and $\hat{u} . \ldots \ldots 41$

3.7. Orientation of proportional tube manifolds in the veto shield. . . . . . 42

3.8. The distribution of coordinate differences shield hit and extrapolated central detector track, for all directions $\mathrm{X}, \mathrm{Y}$ and $\mathrm{Z}$. . . . . . . . 43

3.9. The distribution of angular separation between muon tracks within muon bundles, $\theta_{i j}$, angle between two muons within a pair. . . . 43

3.10. Muon track $\mathrm{AB}$ traversing an $M_{\perp}$ combination of veto shield manifolds. Manifolds QR and PS are orthogonal. . . . . . . . . . 45

3.11. Muon track $\mathrm{AB}$ traversing an $M_{\|}$combination of veto shield manifolds. Manifolds QR and PS are parallel. . . . . . . . . . . . 46

3.12. Number of $M_{\perp}$ and $M_{\|}$combinations for shield surfaces. . . . . . . 47

3.13. Track lengths within the central detector for CLASS 1 muons. . . . . 50

3.14. Track lengths, enclosed by the shield. . . . . . . . . . . . . 50

3.15. Intermuon separation, calculated using Central Dciector tracks. . . . 51

3.16. Intermuon separation, calculated using Central Detector and Veto Shield tracks. ................... 51

3.17. Observed multiplicity (a), (d) from the central detector; (b), (e) from the central detector(CLASS 1) and shield (CLASS 2); (c), (f) from central detector, shield and in-time isolated splats (CLASS 4). Figs. (a), (b) and (c) are for the 1990 data, and Figs. (d), (e) and (f) are for the 1991 data. . . . . . . . . . . . 52

3.18. Three dimensional view of an event. The numbered tracks are examples of the various classifications of candidate muon tracks. . . . 56 
3.19. An Event Display shiwng the 3-D view together with the layout (unfolded) of veto shield superpanels. . . . . . . . . . . . 57

3.20. An Event Display showing the coincident hit patterns recorded on some veto shield surfaces with two muon tracks. . . . . . . . . 58

3.21. An Event Display showing the coincident hit patterns recorded on all veto shield surfaces in a twelve multiple muon event. . . . . . . 59

3.22. STING image of an event which has fourteen muons passing through ine central detector. . . . . . . . . . . . . . 66 60

4.1. Coordinate system for the Soudan-2 cavern: $\beta$ is the zenith angle and $\phi$ is the azimuthal angle. . . . . . . . . . . . . 68

4.2. Distributions of zenith angle for muon bundles. . . . . . . . . 68

4.3. Muon azimuthal angle distributions for 1990 and 1991 data samples.

The distributions are integrated over all zenith angles. . . . . . . 69

4.4. N.uon azimuthal angle distributions binned according to zenith angle, for 1990 and 1991 data samples. . . . . . . . . . . . . . 70

4.5. Shape of the terrain above the Soudan 2 detector. (All distances are in meters.) . . . . . . . . . . . . . . . .

4.6. Errors on $R_{i j}$ (a) associated with pairs of central detector tracks, (b) with combinations involving an $M_{\perp}$ and an $M_{\|}$candidate muons, (c) with pairs of $M_{\perp}$ candidate muons only, and (d) with pairs of $M_{\|}$candidate muons only. . . . . . . . . . . . 7

5.1. All-particle energy spectrum for four models. The total flux is multiplied by $E^{2.5} \ldots \ldots \ldots \ldots \ldots 77$

5.2. Muon multiplicity distributions from each of the four CR composition models. ..................... 80

5.3. Lateral spread among bundle muons, measured from the bundle core, from the four models. . . . . . . . . . . . . . . . . . 82 
5.4. Fractional composition versus primary energy for composition models (a) Linsley, (b) CMC, (c) Maryland, and (d)P-poor. The solid curves in all figures give the contributions of $p$ and Fe. Contributions from alpha particles, from the CNO group, and from the intermediate mass nuclei $\mathrm{Mg}$ and $\mathrm{Si}$, are depicted by dashed, dotted, and dot-dashed curves respectively. . . . . . . . . 86

6.1. The zenith angle distribution, compared to the CMC model. The arrow indicates where the zenith angle cut has been made. The central detector triggering inefficiency is clearly seen between $0^{0}$ and $15^{\circ} \ldots \ldots \ldots \ldots \ldots \ldots \ldots \ldots \ldots$

6.2. Intermuon separation recorded in the central detector, as observed in the data and as predicted by the CMC model. . . . . . . . . 95

6.3. Number of hits versus the number of shield layers responding to a track, for the data and for the simulation (solid and dashed histograms respectively). In Fig. 6.3(a), the two layer hits with no hits on one end are include ${ }^{2} . \ldots \ldots \ldots$. . . . . . . 97

6.4. Distribution of muon bundle multiplicity, compared to expectations of four cosmic ray composition models. . . . . . . . . . . . 99

6.5. Distribution of muon bundle multiplicity, compared to estimated total multiplicity which includes some of the tracks from Group/No hit, Group/Single, Single/No hit and Single/Gap categories. . . 100

6.6. Muon yield at $2090 \mathrm{mwe}$, proton versus iron primaries. . . . . . . . 102

6.7. Relative rates for bundle multiplicity as a function of primary energy:

(a) Mixture of nuclei (CMC model); (b) primary protons; (c) primary iron nuclei. . . . . . . . . . . . . . . . . 104 
6.8. Comparison of muon bundle rates as a function of multiplicity, for the four composition models using a rock density of $2.93 \mathrm{~g} \mathrm{~cm}^{-3}$ and using the $a$ and $b$ from Soudan 1. Absolute rates for the models have to be multiplied by the factor $\eta=1.4$ to compare with the data. . . . . . . . . . . . . . . . 108

6.9. Multiple muon bundle rates using the CMC model (a) for three different rock aensities and using $a$ and $b$ and (b) for the density $2.80 \mathrm{~g} \mathrm{~cm}^{-3}$ and using $a_{2}$ and $b_{2} \ldots \ldots \ldots$. . . . . . . . . 109

6.10. Comparison of muon bundle rates as a function of multiplicity, for the four composition models. Events for models were generated using the rock density $2.80 \mathrm{~g} \mathrm{~cm}^{-3}$ and using $a_{2}$ and $b_{2} \ldots \ldots 110$

6.11. Distribution of separations between pairs of muons, in the data and in the models. . . . . . . . . . . . . . . . . 112

6.12. Distribution of separations between pairs of muons, for the models only. 112

7.1. Average muon multiplicity versus zenith angle. The data is compared to the four models of primary cosmic ray composition. . . . . . 114

7.2. Intermuon separation versus zenith angle for bundle multiplicities 2 and 3, compared to the predictions of the composition models. . 117

7.3. Intermuon separation versus zenith angle, for multiplicities $N_{\mu} \geq 4$, compared to the composition models. . . . . . . . . . . . . 118

7.4. (a) The average intermuon separation versus zenith angle for three different experiments; (b) the average intermuon separation versus depth. . . . . . . . . . . . . . . . . . 119

7.5. Intermuon separation versus bundle multiplicity; the data compared to predictions of the four composition models. . . . . . . . . . . 123

7.6. Multiplicity distributions for data and for the CMC model with $p$ and Fe primaries only (normalized to the number of two muon events in the data sample). . . . . . . . . . . . . . 125 
7.7. (a) Average multiplicity versus zenith angle for data and for CMC model with $p$ and Fe primaries; (b) average multiplicity versus the average intermuon separation for the same model. . . . . . 126

8.1. Fractional cosmic ray composition; world data . . . . . . . . 133

C.1. Distance of perpendicular separation between two parallel muon tracks 144

D.1. HPW chamber cross-section . . . . . . . . . . . . . . . 146

D.2. Schematic diagram for one channel. . . . . . . . . . . 148

D.3. Board layout for one channel in detail and for entire board. . . . . . 149 


\section{Chapter 1 \\ Cosmic Rays}

\section{Introduction}

Since the discovery of cosmic rays early in this century, extensive experimental effort has been devoted to the study of their composition and origin. A wide variety of techniques are being used around the world to examine the flux of cosmic radiation over the full range of its rapidly-falling energy spectrum. Balloon and satellite experiments have been used for direct studies of the low energy component of the primary cosmic ray flux. Surface arrays and underground particle detectors have been used to study indirectly the high energy component of cosmic radiation which extends up to $10^{8} \mathrm{TeV}$ per nucleus in energy incident upon the upper atmosphere. The purpose of this dissertation is to present new experimental observations and analysis relevant to the nuclear composition of cosmic rays with incident energy range between $10 \mathrm{TeV}$ and $10^{5} \mathrm{TeV}$, utilizing the Soudan 2 underground nucleon decay detector.

Primary cosmic ray nuclei collide with oxygen and nitrogen nuclei of the upper atmosphere, producing charged and neutral mesons that decay into photons, electrons, muons, and neutrinos. While the photons and electrons can be detected only in the lower atmosphere or at the Earth's surface, the muons and the neutrinos, which are highly penetrating, can also be detected underground. Our approach is to study the "bundles" of coincident muons which are observed deep underground, in order to infer properties of the primary cosmic ray nuclei. We compare samples of muon bundle events (multiple muon events) with existing cosmic ray nuclear 
composition models to examine whether the primary cosmic rays consist predominantly of either "light" or "heavy" nuclei within the incident energy range under consideration.

\subsection{Origin of Cosmic Rays and Their Composition}

Cosmic rays are ionized nuclei which are incident at high velocities upon the Earth's upper atmosphere. This "primary cosmic radiation" includes protons and alpha particles $\left({ }_{2} \mathrm{He}^{4}\right)$ together with heavier nuclei such as $\mathrm{C}, \mathrm{N}, \mathrm{O}, \mathrm{Si}, \mathrm{Mg}$, and Fe. The primary cosmic rays are produced and subsequently accelerated by energetic astrophysical sources beyond the solar system. Candidate sources include supernova, pulsars, active galactic nuclei, quasars, and radio galaxies [1]. The flux of primary cosmic rays falls rapidly as one goes from the low incident energy of $\leq 100 \mathrm{GeV}$ to $\geq 10^{10} \mathrm{GeV}$ (see Fig. 1.1). The falloff can be expressed by a power law,

$$
\frac{d N}{d E}=K E^{-\gamma}
$$

where the normalization factor $K$ and the spectral index $\gamma$ are approximately constants.

Fig. 1.1 shows the differential cosmic ray flux obtained from a variety of experiments [1]. It is evident in the figure that the slope of the spectrum changes between $10^{3} \mathrm{TeV}$ and $10^{4} \mathrm{TeV}$ of incident energy per nucleus. This region is called the "knee" of the energy spectrum. The spectral index $\gamma$ is about 2.6 for the low energy regime extending up to $10^{4} \mathrm{TeV}$, while for the higher energies $\gamma \approx 3.0$. The primary cosmic rays with energies around the knee region are thought to originate within our galaxy; however, the nuclear composition in this region has not been ascertained. Another, less pronounced, slope change occurs around primary energies of $10^{7} \mathrm{TeV}$ and this region is called the "ankle". This Thesis presents new measurements and analysis which have bearing on the relative abundance of nuclei in cosmic rays which are below and at the knee region. 


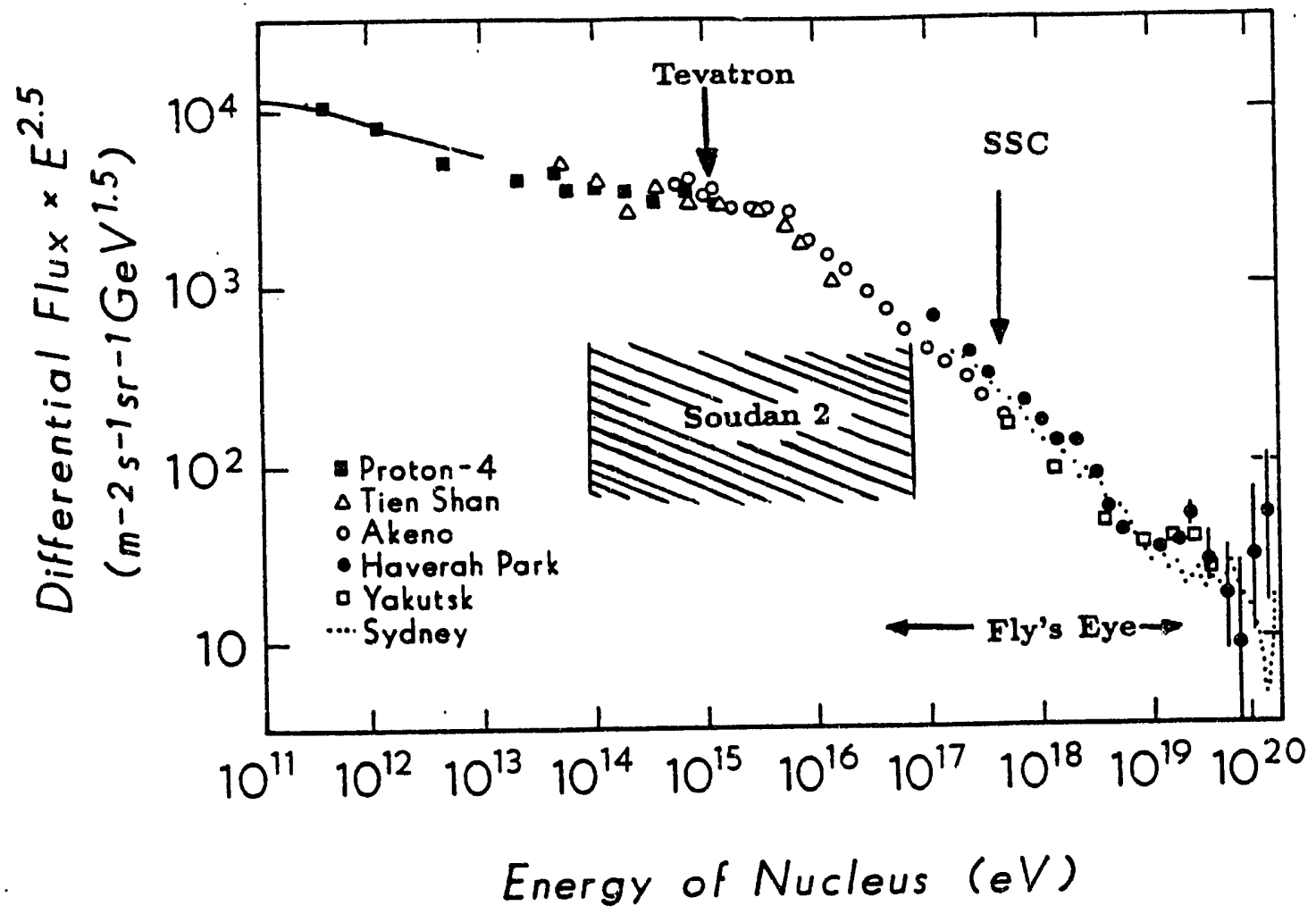

Figure 1.1: The All Particle Energy Spectrum

A substantial fraction of cosmic rays with $E>10^{4} \mathrm{TeV}$ (above the knee region) are hypothesized to originate in the cores of Active Galactic Nuclei (AGN). According to the model proposed by R. J. Protheroe and A. P. Szabo [2], AGNs may be the most important source of cosmic rays at and beyond the knee region. The AGN-produced cosmic rays are predicted to be predominantly protons. Heavier nuclei such as iron can also be accelerated within AGNs, but they will not escape intact from the AGNs fiery, dense central regions. Instead, they will be broken up into lighter particles as a result of interactions with high energy photons.

It is informative to superimpose a scale that is man-made upon the cosmic energy scale. In Fig. 1.1 the arrows show the highest available equivalent fixed-target beam energies in existing accelerators (the Fermilab Tevatron) and in accelerators under construction (SSC). In comparison, the shaded areas indicate cosmic ray energies which are indirectly accessible by Soudan 2 and by some other existing experiments such as the MACRO [3] underground detector and the Fly's Eye air Cherenkov 
shower array [4]. It can be seen that, the cosmic ray experiments are exploring the behavior of interactions which are more energetic than the accelerator experiments.

\subsection{Study of Cosmic Rays: A Historical Perspective}

Cosmic ray research began at the turn of the centurv, when ionization and electrical conduction of gases irradiated by cosmic ray particles were investigated. During the early 1900 s, $\alpha$ - particles, $\beta$ - particles and $\gamma$ - rays were discovered by studying naturally radioactive elements using electroscopes. Ii was observed that electroscopes kept discharging even when kept away from radioactive material. The latter ionization appeared to be due to radiation coming in from the atmosphere or from outer space. The situation was clarified in 1912, when Hess and co-workers ascended by balloon to nearly 6 kilometers above the Earth's surface and observed the cosmic radiation more directly [5]. The radiation was found to consist mostly of protons but to contain nuclei of mass numbers up to 56 ( Fe). Later, even heavier nuclei such as $\mathrm{Nd}(\mathrm{Z}=60)$, and $\mathrm{Th}(\mathrm{Z}=90)[6]$ were detected. Since Hess's early work, many experiments have been carried out using cosmic rays. The positron (anti-electron) was discovered in 1933, confirming Dirac's theory of anti-matter. Then " $\mu$ - mesons", initially considered to be the Yukawa mesons, and afterwards $\pi$-mesons, the true mediators of the nuclear force, were discovered. In the late 1940s and early 1950s, particles such as the $K$ mesons and $\Lambda, \Sigma$, and $\Xi$ hyperons were discovered in the cosmic radiation using the nuclear emulsion technique.

With the development of experiments carried aloft by rockets and satellites, more detailed information on cosmic rays of relatively low energy was obtained during the 1960s. To study high energy cosmic rays however, detectors of large area are required since the high energy cosmic ray fluxes are small. Currently, large surface arrays and underground experiments are used to study high energy cosmic rays in indirect ways. Measurements which are feasible with underground detectors will be discussed 


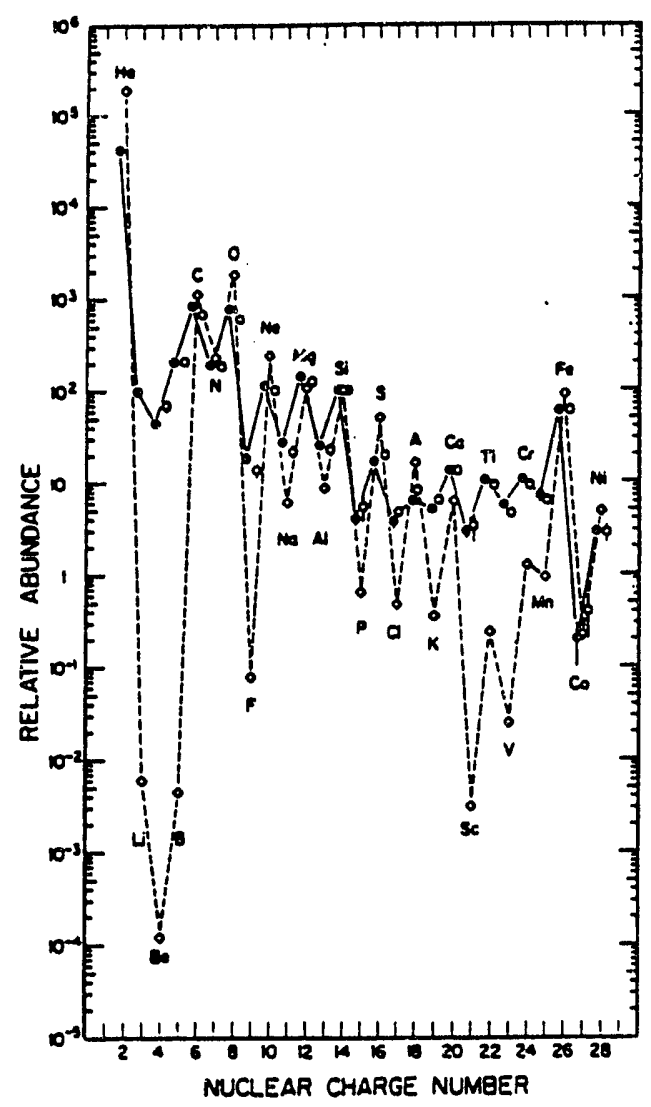

Figure 1.2: Solar and Cosmic Ray abundances. Solid circles and open circles show cosmic rays for low (up to $0.3 \mathrm{GeV}$ ) energy and high (up to $2 \mathrm{GeV}$ ) energy repectively; open diamonds show solar abundances; all are relative to the abundance of silicon.

in section 1.6.2.

\subsection{Solar and Cosmic Ray Relative Abundances}

A comparison of the relative abundances of elements found in cosmic rays, which presumably originate outside of our solar system, with abundances observed within the solar system, is shown in Fig. 1.2 [7]. Overall, the relative compositions are quite similar, but some differences are apparent. The percentages of hydrogen and helium are a little lower in the cosmic rays than in the solar system. The odd-even effect in the relative nuclear stability (nuclei with even numbers of nucleons are more stable and more abundant than those with odd numbers of nucleons) is also seen in both solar and cosmic ray abundances. Cosmic rays are richer in two groups of elements, namely those with mass numbers 7 to 11 ( $\mathrm{Li}, \mathrm{Be}$, and $\mathrm{B}$ ), and those with 
mass numbers 45 to 55 ( $\mathrm{Sc}, \mathrm{Ti}, \mathrm{V}, \mathrm{Cr}$ and $\mathrm{Mn}$ ), due to spallation of $\mathrm{C}, \mathrm{O}$ anu of $\mathrm{Fe}$ nuclei of the cosmic rays during the passage through the intersteller medium [7] [8].

It is believed that all chemical elements in the solar system and in the cosmic rays originate either with the primordial nucleosynthesis or with violent episodes in stellar evolution. For example, ${ }^{2} \mathrm{H}$ and ${ }^{3} \mathrm{He}$, which are found in the solar system, are probably from the primodial nucleosynthesis [9] that occurred within 3 to 4 minutes after the Big Bang. At this early epoch, protons and neutrons could combine together to $\mathrm{p}$.oduce ${ }^{4} \mathrm{He}$ through a chain of nuclear reactions such as $p+n \rightarrow{ }^{2} \mathrm{H}+\gamma$, ${ }^{2} \mathrm{H}+n \rightarrow{ }^{3} \mathrm{H}+\gamma,{ }^{2} \mathrm{H}+p \rightarrow{ }^{3} \mathrm{He}+\gamma,{ }^{3} \mathrm{H}+p \rightarrow{ }^{4} \mathrm{He}+\gamma,{ }^{3} \mathrm{He}+n \rightarrow{ }^{4} \mathrm{He}+\gamma$. Somewhat heavier nuclei such as ${ }^{7} \mathrm{Be}$ and ${ }^{7} \mathrm{Li}$ could also be formed during the primordial nucleosynthesis. About $10^{6}$ years later, the temperature was low enough to allow formation of neutral atoms with the caft!re of electrons by nuclei. At this epoch gravity began to play an essential role in the formation of galaxies and of protostars (material about to condense into a star) within the galaxies.

As a protostar contracts gravitationally, it heats up. Eventually hydrogen fusion, the process by which a star converts $H$ into He, will start - a star is born. Billions of years later, when the hydrogen in the core of the star is exhausted, further gravitational contraction occurs and the temperature increases. Helium is converted into carbon $\left(3^{4} \mathrm{He} \rightarrow{ }^{12} \mathrm{C}\right)[10]$. The temperature keeps increasing and the fusion sequence $\mathrm{C} \rightarrow \mathrm{O}, \mathrm{Ne}, \mathrm{Mg} \rightarrow \mathrm{Si} \rightarrow \mathrm{Fe}$, will occur. This entire chain of processes is called stellar nucleosynthesis. The mass of the star's core increases due to the formation and accumulation of iron. If there arises a time when the electron degeneracy pressure of the core can no longer support the overlying layers together with the core's own weight, gravity takes over and a catastrophic collapse occurs which results in a "supernova" explosion of the star. When the collapse occurs, the matter in the core dissociates into protons, neutrons, and electrons. The density of the compressed core increases to nearly five times the density within an ordinary atomic nucleus. Only a few of the neutrinos which are created during the collapse escape at this 
point because of high density. The collapsing matter apparently bounces back and generates a shock wave; the bounce mechanism is not understood. The inner core expands and the spherical shock wave travels outward with high speed $\approx 18,000$ $\mathrm{km} / \mathrm{sec}$. More neutrinos escape from the core as the density of the core decreases. The shock wave sweeps the steller matter outward, ejecting it into the interstellar medium. Thus supernova explosions provide a mechanism for the distribution of heavy elements into the cosmic rays, and into matter which eventually coalesced to form our solar system.

\subsection{Acceleration Mechanisms}

Many mechanisms for cosmic ray acceleration have been proposed, however most fall into one of two categories: (i) Statistical acceleration, first proposed by Fermi (see below) which leads to a power law for the energy spectrum; and (ii) direct acceleration mechanisms which yield good predictions for high energy cosmic rays in the energy region up to $10^{6} \mathrm{TeV}$. Although much progress has been made with theoretical investigation of the acceleration mechanisms, there is none at present which gives a completely satisfactory picture.

In the first version of Fermi's acceleration model [5], the acceleration hinges upon the reflection of charged particles by the galactic magnetic field (order of $10^{-6} \mathrm{G}$ ). In each collision of a charged particle with the magnetic gas cloud, the particle gains some energy from the magnetic field as a result of the fact that the kinetic energy of the massive cloud is much larger than the kinetic energy of the cosmic ray particles colliding with it. With successive colisions, a cosmic ray particle can acquire enough energy from the massive clouds to be accelerated to high energies [1].

Consider collisions along in axis (one-dimensional) wherein a cosmic ray particle of mass $m$ moving with velocity $v$ collides with a magnetic cloud of mass $M$ moving with velocity $V$. The collision can be either "head-on" or "following". The 
probability for a head-on collision is $(v+V) / 2 v$, while for a following collision it is $(v-V) / 2 v$. There is a greater probability for a head-on collision; there is an energy gain for each heau -on collision and the rate of energy gain is given by [5]

$$
\frac{d E}{d t}=\alpha E \text {. }
$$

Assume that the cosmic ray particle stays within the magnetic cloud for a time $\tau$. If $N(E)$ is the number of cosmic ray particles accelerated with energy $E$, particle diffusion calculations [5] lead to an equation for $N(E)$ which is given by

$$
N(E)=\text { constant } \times E^{-\left(1+\frac{1}{a \tau}\right)} .
$$

But this is just the power law of the cosmic ray energy spectrum! In this model, the cloud velocity $V$ is small, and the mean free path for collision of cosmic ray particles is long. Consequently there arise several undesirable features with this model: (i) The acceleration is slow and an extended region of space is required; (ii) the energy loss due to ionization is not taken into account when the energy gain is calculated; (iii) even though the model leads to the power law for energy spectrum, it fails to predict why the spectral exponent should be close to 2.5 .

A second version of Fermi's acceleration mechanism, developed independently by Bell, and by Blandford and Ostriker [5], is based upon the propagation of shock waves from supernova through the intersteller medium. The velocity of these shock waves is much greater than the speed of sound in the medium. When a cosmic ray particle passes through a shock wave, it acquires kinetic energy from the gas behind the shock. When this occurrence is repeated many times the cosmic rays eventually gain substantial energy. This mechanism predicts the exponent in the power law to be " 2 " which is not too far from the observed index 2.5 in the energy spectrum. With this mechanism, cosmic rays can achieve energies of up to $10 \mathrm{TeV}$.

With direct acceleration mechanisms, high energy cosmic rays are predicted to acquire energies of up to $10^{6} \mathrm{TeV}$. Sources for direct acceleration include rotating objects having high magnetic fields such as neutron stars [1]. Their magnetic field is 
typically $10^{12}$ gauss, and they rotate with nearly 30 revolutions per second. When a charged cosmic ray particle passes through the rotating magnetic field surrounding a neutron star, the time-varying magnetic flux (through a surface bounded by the particle's trajectory) produces an electromotive force (emf) which accelerates the particle. The Faraday-induced emf can accelerate the particle to energies up to $10^{19}$ $\mathrm{eV}$ [5]. This model unfortunately, does not predict the power law for the cosmic ray energy spectrum.

Other direct acceleration models have been proposed which predict cosmic rays to have energy $10^{8} \mathrm{TeV}$. Such models typically relate cosmic ray physics to cosmological theories of the evolution of universe.

\subsection{Direct and Indirect Methods for the Study of Primary Cosmic Rays}

Since the cosmic ray flux falls off rapidly with increasing energy (see Fig. 1.1), different techniques have to be used at different energies. At the lowest energies $(\approx$ $100 \mathrm{GeV}$ range) one can detect one particle per $\mathrm{m}^{2}$ per second; however the flux is one particle per $\mathrm{km}^{2}$ per century at the highest energy range $(\approx 1 \mathrm{EeV})^{1}[1]$. Direct detection techniques which utilize balloons or satellites can be used to study the lower energy part of the cosmic ray spectrum (up to several $100 \mathrm{TeV}$ ). Surface arrays and underground detectors are widely used to study the high energy $(>1$ $\mathrm{TeV}$ ) component of the cosmic rays. In the latter experiments, secondary cosmic rays are used to study the primary component; such studies are referred to as the "indirect approach". The direct and indirect approaches will be discussed in the following two sections.

\footnotetext{
${ }^{1} 1 \mathrm{EeV}=10^{6} \mathrm{TeV}$
} 


\subsubsection{Direct Methods}

Direct methods allow study of primary cosmic rays up to about $200 \mathrm{TeV}$. Below this energy the cosmic ray flux is high enough to allow studies utilizing airborne calo-imeters, ionization chambers, scintillators, emulsion stasks, Cherenkov detectors and transition radiation detectors. These detectors are taken aloft either by balloons or by satellite to study primary cosmic rays directly, before they collide with air nuclei in the upper atmosphere. There are of course disadvantages to using balloons and satellites: The detector size (effective area) is limited; and the exposure time depends upon the flight time.

Two major experiments stand out among all of the direct studies. One was the University of Chicago "egg", a detector consisting of scintillators, transition radiation detectors, gas cherenkov and multi-wire proportional chambers. Data was collected during a spacerraft's flight from July 29 to August 6, 1985 [7]. About 100,000 particles were observed with energies of about one or two $\mathrm{TeV}$ traversing the detector while it was in orbit. The detector had been designed to observe heavy nuclei with electric charge above a threshold of $Z=6$ (C) with energies of up to $100 \mathrm{TeV}$.

The other experiment was carried out by a Japanese-American collaboration known as "JACEE" [11]. This was a balloon experiment; it detected cosmic räv's within au energy range similar to the one probed by the Chicago egg.

Study of the cosmic rays above the hundred $\mathrm{TeV}$ range requires detectors with large area and with long exposure time, neither of which can currently be achieved by balloon or satellite experiments. Consequentry, researchers have had to rely on indirect methodo to study the high energy cosmic rays. 


\subsubsection{Indirect Methods: Secondary Cosmic Rays}

Primary cosmic rays incident upon the upper atmosphere interact mostly with nitrogen and oxygen nuclei in the air, producing charged and neutral particles which are called "secondary cosmic radiation". Included are small numbers of nucleons and nuclear fragments, together with much larger numbers of mesons $\left(\pi^{ \pm}, \pi^{0}, K^{ \pm}, K^{0}\right)$. These secondary particles may undergo further ccllisions leading to a "hadronic cascade", or they may decay. Muons are produced mainly through $\pi^{ \pm} \longrightarrow \mu^{ \pm} \nu$ and $K^{ \pm} \longrightarrow \mu^{ \pm} \nu$ decays. Most of the low energy muons decay into electrons, positrons and neutrinos: $\mu^{ \pm} \longrightarrow e^{ \pm}+\nu_{e}\left(\bar{\nu}_{e}\right)+\bar{\nu}_{\mu}\left(\nu_{\mu}\right)$. An "electromagnetic cascade" develops starting with a $\pi^{0} \longrightarrow \gamma \gamma$ decay followed $\gamma \longrightarrow e^{+} e^{-}$conversions, where the electrons generate more photons by bremsstrahlung. Such electromagnetic processes happen again and again, generating an extensive e'ectromagnetic shower. The aggregate of all secondary particles is an "Extensive Air Shower" (EAS), illustrated in Fig. 1.3.

Muons are highly penetrating particles since they have no strong nuclear interaction with matter. Since many of the muons have velocities approaching the speed of light, relativistic time dilation allows energetic muons to be detected on the surface of the Earth and also deep underground. For relativistic particles the flight length before decay is $\beta \gamma c \tau$, where $\tau$ is the lifetime of the particle. The average flight length for a $100 \mathrm{GeV}$ muon (in the laboratory frame) is $750 \mathrm{~km}$, to be compared to about 5 $\mathrm{km}$ for $100 \mathrm{GeV}$ pions. The difference reflects the fact that the charged pion lifetime $\left(2.6 \times 10^{-8} \mathrm{~s}\right)$ is distinctly shorter than the muon lifetime $\left(2.2 \times 10^{-6} \mathrm{~s}\right)$. Note that the depth of the atmosphere is between 30 and 40 kilometers. As a consequence, it is possible to observe muons deep underground.

There are two indirect methods to study cosmic rays, utilizing observations either of the electromagnetic cascade or of the muon flux. Arrays on the surface of the Earth typically investigate the electromagnetic cascades either at the surface or in the atmosphere (Cherenkov technique), and also the low energy muon component. Underground detectors are used to study the high energy muon component. By 

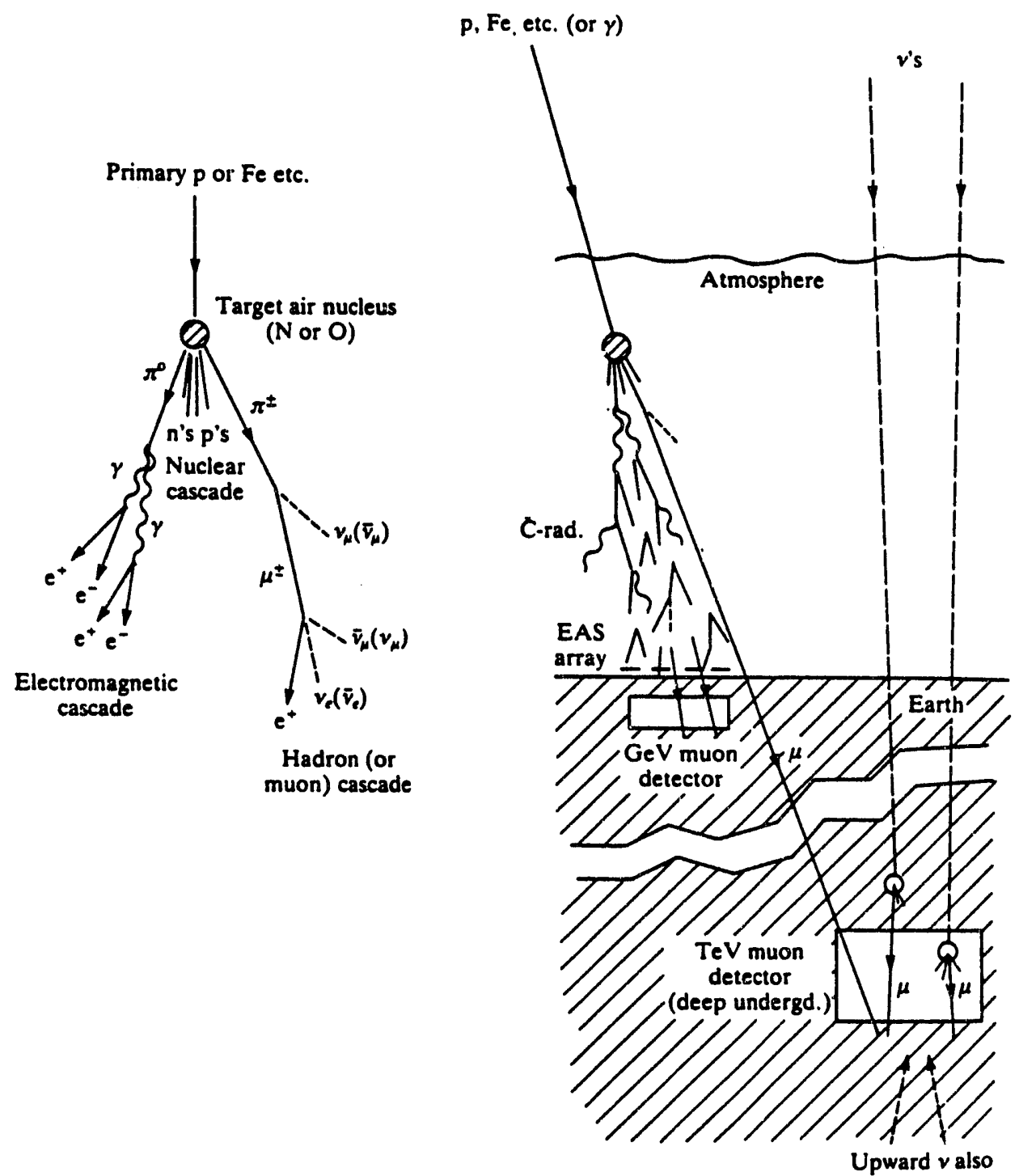

Figure 1.3: The development of secondary cosmic rays and their detection.

studying the cosmic ray secondaries on the surface and underground, one can infer properties of primary cosmic rays which have energies above $1000 \mathrm{TeV}$.

\subsection{Detectors to Study Secondary Cosmic Rays}

Nuclear emulsions, cloud chambers, gas filled detectors, proportional chambers, photomultiplier tubes and scintillation detectors have been used to observe secondary cosmic rays. The underground detectors, which are principally built for proton decay 
and for magnetic monopole searches, can also be used to study muons. Detectors deployed on the surface of the Earth or underground can be built with large area and are not subject to exposure time limitation. Large areas and longer exposure time are of course needed to study ultra high energy cosmic rays because of very low cosmic ray flux in this energy range.

\subsubsection{Surface Arrays}

There are many surface arrays around the world. Among the largest area cosmic ray surface arrays are Volcano Ranch-USA $\left(8 \mathrm{~km}^{2}\right)$, the Sydney array-Australia (34 $\left.\mathrm{km}^{2}\right)$, Haverah Park-UK $\left(11 \mathrm{~km}^{2}\right)$ and the Yakutsk array-USSR $\left(35 \mathrm{~km}^{2}\right)$ [5]. These "extensive air shower arrays" utilize a variety of detector technologies, including scintillators, proportional tubes, and Cherenkov counters. From the shower size and other properties of the secondary cosmic radiation one can infer properties of the primary cosmic radiation, but the analysis is not straightforward. Particle interactions in the primary collisions are assumed to have features as observed in high energy accelerator experiments. Unfortunately, for high energy collisions in the $\mathrm{PeV}\left(=10^{3} \mathrm{TeV}\right)$ energy range there are no corresponding accelerator-based data, consequently the modelling of interactions involving both nucleus-nucleus and nucleon-nucleus collisions necessarily requires extrapolations whose level of accuracy is difficult to assign.

The University of Utah "Fly's Eye" utilizes a different technique. Its 880 skywardturned phototubes [1] detect fluorescence emitted by extended air showers. Recorded is the total integrated light, as well as the relative time of light arrival at individual phototubes spaced approximately $3.5 \mathrm{~km}$ apart. The experimenters measure the distribution of $X_{\max }$, the distance from the top of the atmosphere to the shower maximum, and utilize this information to infer the composition of cosmic radiation. The latest analysis of Fly's Eye data by Gaisser and Sokolsky [12] for energies $10^{5}$ $\mathrm{TeV}$ and $10^{6} \mathrm{TeV}$ (i.e. above the knee) indicates that protons make up only $20 \%$ 
of the cosmic rays, the rest being heavier nuclei. As will be reported in this Thesis, the muon bundle data from Soudan and from other deep underground experiments indicates a relatively "light" nuclear composition for energies below and at the knee. As noted in Ref. [12], a drastic change from light to heavy composition above the knee would be unusual and puzzling.

\subsubsection{Underground Detectors}

A number of large underground detectors have been used to study muon bundles, including Soudan 2 [13] (USA) consisting of a tracking calorimeter completely surrounded by an array of proportional chambers, MACRO [3] (Italy) with streamer tubes and liquid scintillation counters, and Fréjus [14] (French Alps) with flash chambers and Geiger counters (no longer in operation). Earlier experiments used smaller detectors, e.g. Soudan 1 [15] (no longer running), the Kolar gold mine nucleon decay detector [16] (India; to stop in 1993) and the NUSEX (Mont Blanc, French-Italian border) nucleon decay experiment (not running) [17] [18].

The underground detectors were designed primarily for nucleon decay and magnetic monopole searches; however they can also be used to study cosmic rays that penetrate deep into the Earth, namely muons and neutrinos. The energy threshold for a muon to reach an underground site is determined by the rock overburden above the detector. For the Soudan 2 site the threshold for vertical muons is about 0.8 $\mathrm{TeV}$.

By studying the properties of multiple muon events underground such as the muon multiplicity and muon lateral distribution, one can infer - or at least constrain - the relative nuclear abundance within primary cosmic rays. The lateral distribution of muons within multiple muon events depends upon the transverse momentum $\left(P_{\perp}\right)$ of the mesons produced in a primary cosmic ray collision. Large tracking areas $(\approx$ $1000 \mathrm{~m}^{2}$ ) are desirable for good coverage of the lateral distribution.

In the Soudan 2 experiment, the top surface area of the cavern-liner veto shield 
is nearly $420 \mathrm{~m}^{2}$; the area of the ceiling together with the south, east, and west wall surfaces is $1160 \mathrm{~m}^{2}$. The experiment is located deep underground at 2090 meters water equivalent (mwe) vertical depth. Using this detector, high energy primary cosmic rays in the $\mathrm{TeV}$ to $\mathrm{EeV}$ energy range can be studied by investigation of the underground coincident muon bundle events which they produce. 


\section{Chapter 2 \\ Soudan 2 Nucleon Decay Detector}

\subsection{Overview}

The Soudan 2 nucleon decay detector is located in an old iron ore mine at the Soudan Underground Mine State Park in Soudan, Minnesota. The detector, designed and constructed by Soudan 2 collobaration (see Appendix A), is situated at a depth of 722 meters (2090 mwe) on the 27 th level of the mine. The detector consists of two distinct tracking systems, the Central Detector and the Active Veto Shield. The facility is mainly designed to search for nucleon decay. However, the detector is being used for other studies pertaining to the origin and composition of cosmic rays using single and multiple muons, the neutrino flavor content of the atmospheric neutrino flux, and the existence of magnetic monopoles and other slow, heavily ionizing particles. This facility is the second generation of nucleon decay experiments at the Soudan mine; the first detector, Soudan 1, was located on the 23rd level at a vertical depth of 600 meters. Soudan 1 was also used to study cosmic rays, most recently in coincidence with a prototype surface array [15].

The Soudan 2 central detector is a tracking calorimeter which is modular in design, with module dimensions determined by the size of the elevator cage. The modularity allows portions of the detector to be operated while the remainder is being installed. Each module consists of a stack of corrugated steel sheets interleaved with drift tubes which are read out by vertical anode wires and horizontal cathode strips (see section 2.2). The veto shield consists of proportional tube manifolds of lengths between $1.4 \mathrm{~m}$ and $7.9 \mathrm{~m}$ (see sections 2.3 and 2.4). Upon completion, the 


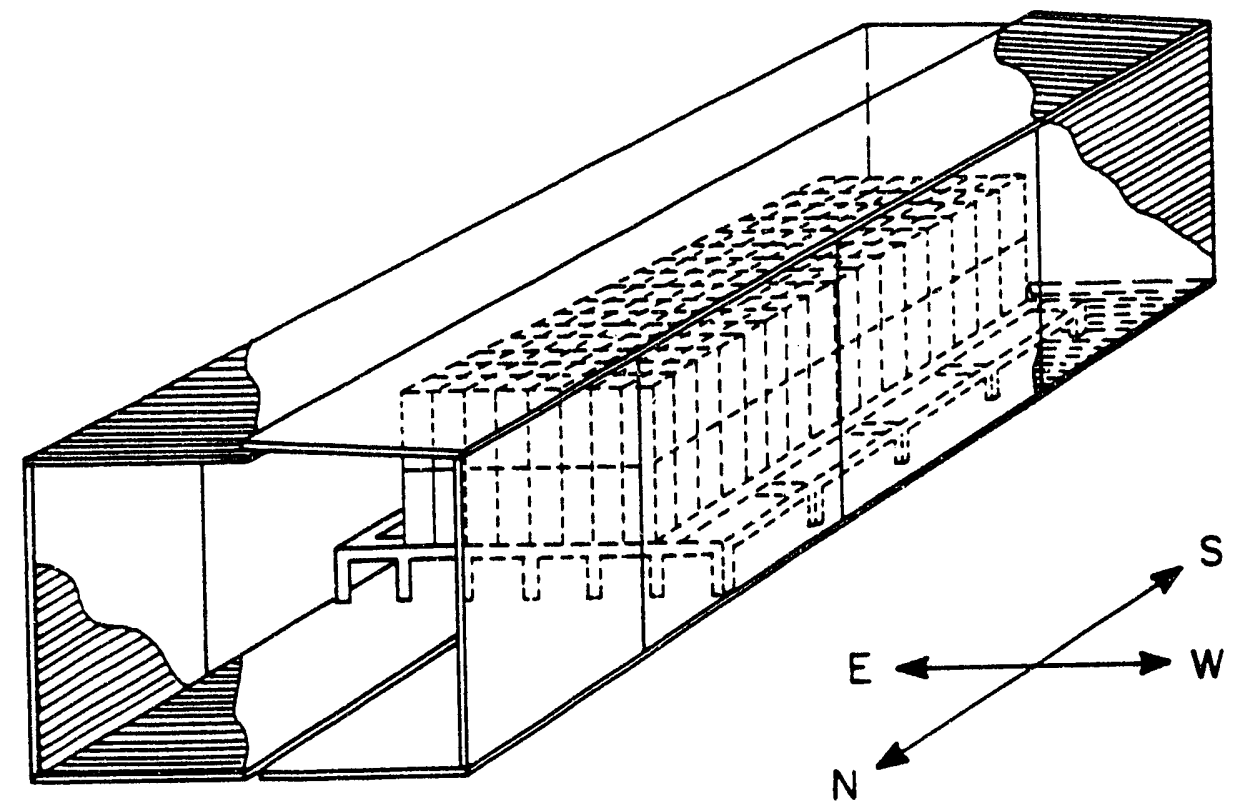

Figure 2.1: Plane view of the Soudan 2 detector.

veto shield will provide nearly $4 \pi$ solid angle coverage around the central detector to enable detection of any charged particles incoming from outside of the Soudan 2 cavern. The veto shield could conceivably be a stand-alone muon telescope if it were able to determine all three spatial coordinates for entering and exiting point of a muon track. This could be accomplished by deploying more proportional chambers in orientations which are transverse to the shield manifolds which are currently operating (see section 2.5).

Construction of both the central detector and the veto shield began in 1985. The first 500 tons of the central detector was completed in the summer of 1990 . Upon completion in 1993, the central detector will have a mass of 960 tons. As of January 1993, the veto shield is nearly completed, with the exception of a few remaining gap-filler sections to be installed on the walls and on the floor. A plane view of the Soudan 2 detector is shown in Fig. 2.1. The stacking of central detector modules is depicted in the middle of the figure; the veto shield array is the rectangular box 
structure surrounding the central tracking calorimeter.

\subsection{The Central Detector}

A brief description of the central cletector is given here. Details of design and performance can be found in references [19], [20] and [21]. The central detector is a fine grained tracking calorimeter. It is built as a stack of standard modules; each module has dimensions of $1.0 \mathrm{~m} \times 1.1 \mathrm{~m} \times 2.7 \mathrm{~m}$ and has mass of 4.3 tons. The total number of modules upon completion of Soudan 2 will be 224 . Modules are arranged into "halfwalls"; each halfwall consists of 8 modules arranged 4 modules across and stacked two modules high. Two halfwalls together constitute one full "wall" of modules, a structure which is 8 modules across, 2 modules high. The readout of ionization from module drift tubes (see below) is organized by halfwalls. Each halfwall can be moved as an entire unit by a transporter which is mounted to the bottom of the detector's "bed" of structural steel.

A schematic drawing of a calorimeter module is shown in Fig. 2.2. A module contains 240 layers of corrugated steel sheets each of which measures $1 \mathrm{~m} \times 1 \mathrm{~m}$ $\times 1.6 \mathrm{~mm}$. The corrugations support $1.5 \mathrm{~cm}$ diameter drift tubes. The drift tubes are sandwiched between mylar sheets which carry copper high voltage electrodes spaced to yield a uniform electric field along the axes of the drift tubes. Additional polystyrene insulation is placed between the mylar and the steel to prevent high voltage breakdown. All drift tubes are filled with pure $85 \% \mathrm{Ar}$ and $15 \% \mathrm{CO}_{2}$ (less than 5 vapour count per million [22] oxygen and other electronegative impurities).

When a charged particle traverses the detector (see Fig. 2.3), it ionizes gas molecules within the individual drift tubes. The liberated electrons drift towards a wire plane consisting of vertical anode wires with high positive voltage (2000 to 2300 volts) and horizontal cathode pads held at ground potential (see Fig. 2.4). Each module has two wire planes on opposite sides facing the ends of the drift tube stack. 


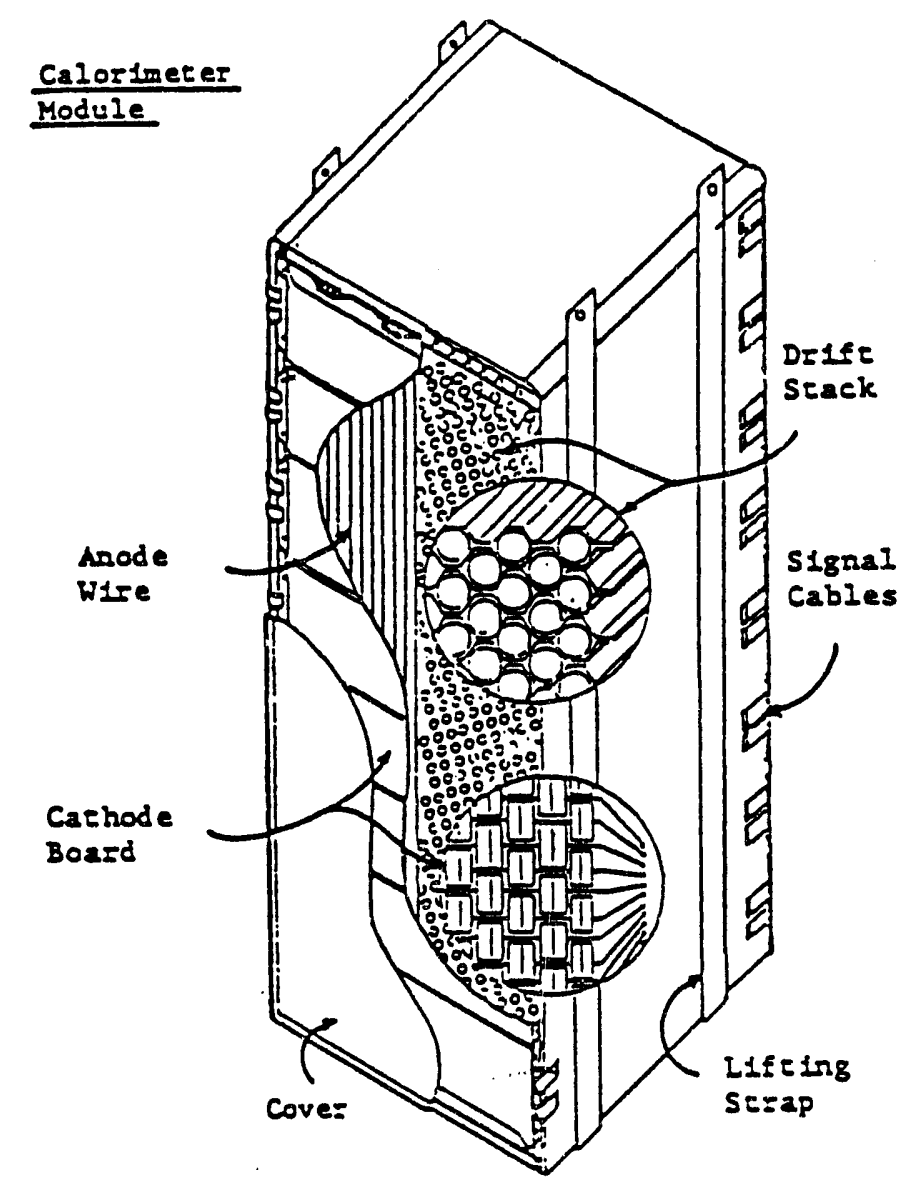

Figure 2.2: A view of a tracking calorimeter module.

The drift ionization exits the tubes whereupon it is collected on the anode wires and imaged on the cathode pads. The resulting signals are then amplified, digitized using a Flash Analog-to-Digital converter, and finally read out. The anode wires and cathode pads give $\mathrm{X}$ and $\mathrm{Y}$ coordinates of the point where the charged particle crossed a particular drift tube. The relative drift time information gives the $\mathrm{Z}$ coordinate for the gas crossing described by $X$ and $Y$. The maximum drift distance along the $\mathrm{Z}$ direction is $50 \mathrm{~cm}$. The drift velocity of an ionized trajectory segment varies from $0.6 \mathrm{~cm} / \mu \mathrm{s}$ to $0.9 \mathrm{~cm} / \mu \mathrm{s}$ and depends on the water content of the gas [19]. The time resolution of the central detector is about $1 \mu$ s. 


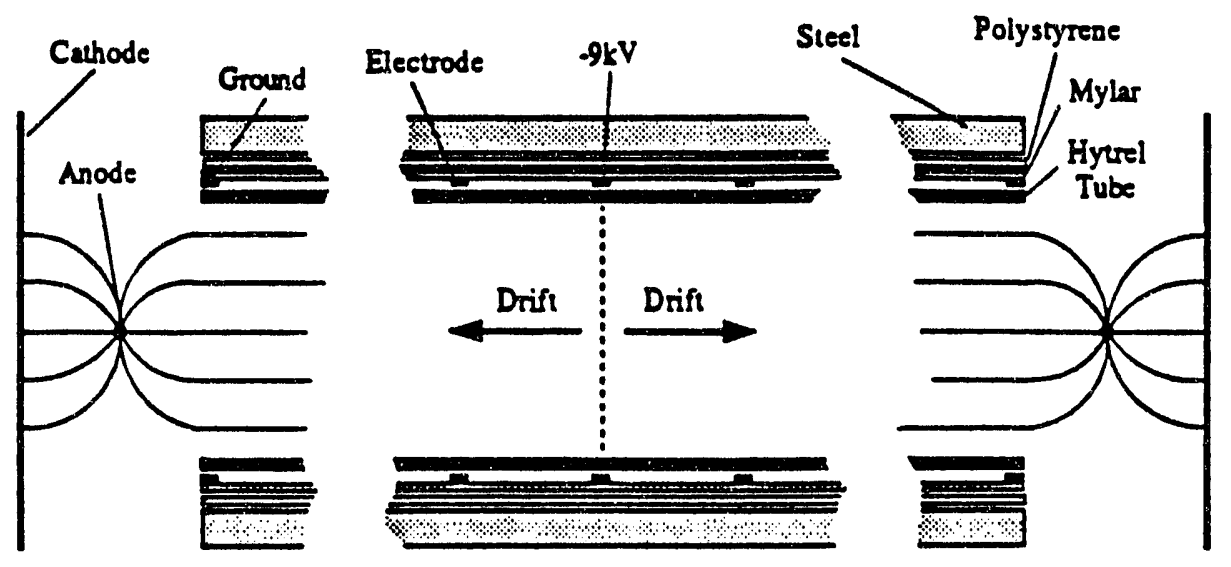

Figure 2.3: Cut away longitudinal view of a drift tube.

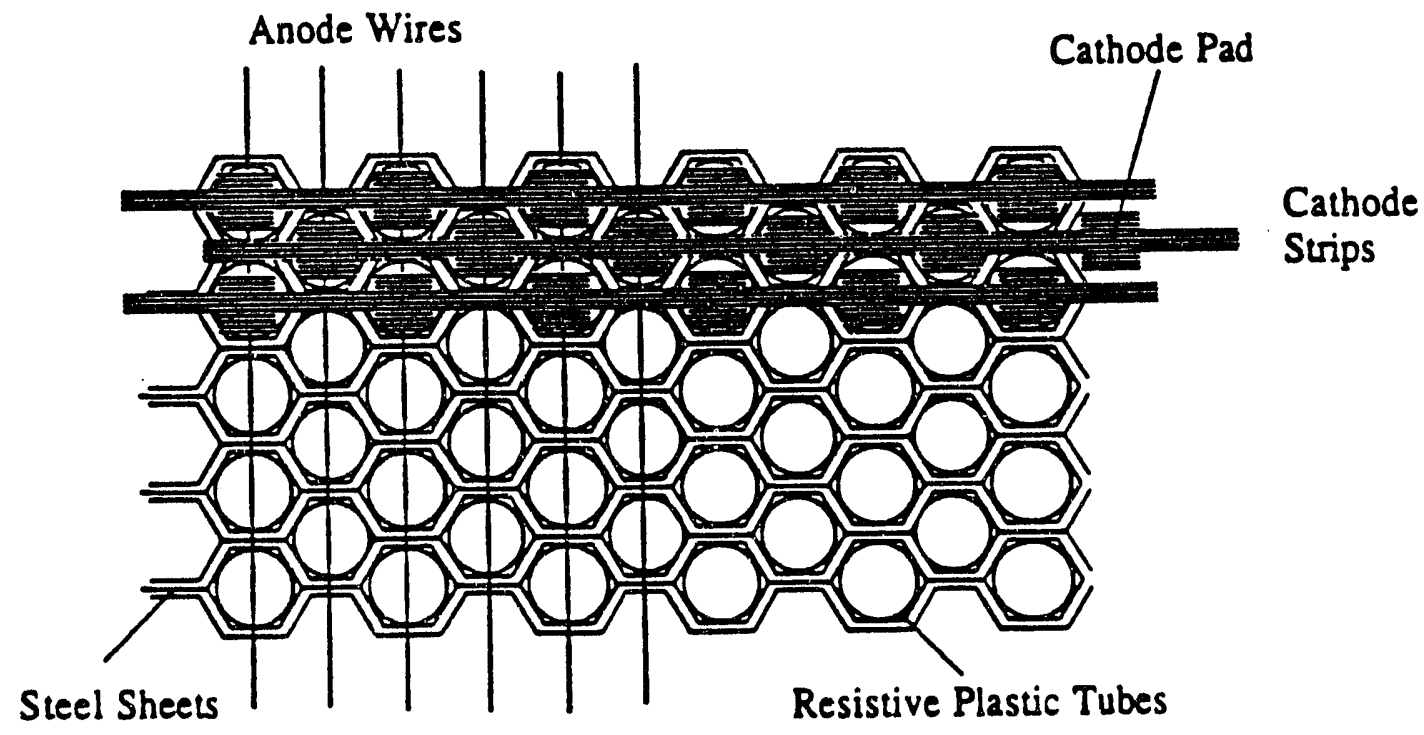

Figure 2.4: A module Wire Plane of the Soudan tracking calorimeter. 
The top surface area of the central detector is about $90 \mathrm{~m}^{2}\left(135 \mathrm{~m}^{2}\right.$ when the modular tracking calorimeter is completely deployed). The calorimeter's spatial resolution is approximately $1 \mathrm{~cm}$ in each of the three spatial coordinates. The tracking calorimeter provides the trigger for occurrence of charged-particle tracks (see section 2.6).

\subsection{The Active Shield}

The active veto shield is an array of nearly 1600 proportional tube manifolds. Each manifold contains eight hexagonal proportional chamber cells. An end view of three manifolds is shown in Fig. 2.5, illustrating how the manifolds interlock so as to avoid detection gaps. A sense wire of $60 \mu \mathrm{m}$ diameter resistive nichrome is strung along the axis of each cell. The preparation of manifolds, stringing of wires, quality control of each manifold and performance characteristics have been descri'sed in Ref. [23].

All four sense wires of the upper cells of a shield manifold (the upper 'layer') are connected in parallel and are read out as a single electronic channel. Similarly, the four wires of the lower layer form a single, independent channel. All wires are kept at positive 2300 volts and all manifolds are filled with a mixture of $95 \% \mathrm{Ar}$ and $5 \% \mathrm{CO}_{2}$. Outputs from both sense wires are fed into a front-end electronics board which is mounted at one end of the manifold within the endcap assembly (see Section 2.4.1).

Sets of adjacent manifolds of the same length constitute veto shield 'panels', each with a given number. For example, the entire east ceiling is regarded as one panel, namely panel \# 7. The shield floor, on the other hand, is divided up into many relatively small panels, reflecting the circumstance that the supporting steel structure penetrates the floor elevation in many places. Within a panel, each marifold is assigned a 'tube' number. Out of the two layers, the layer which is facing the central 


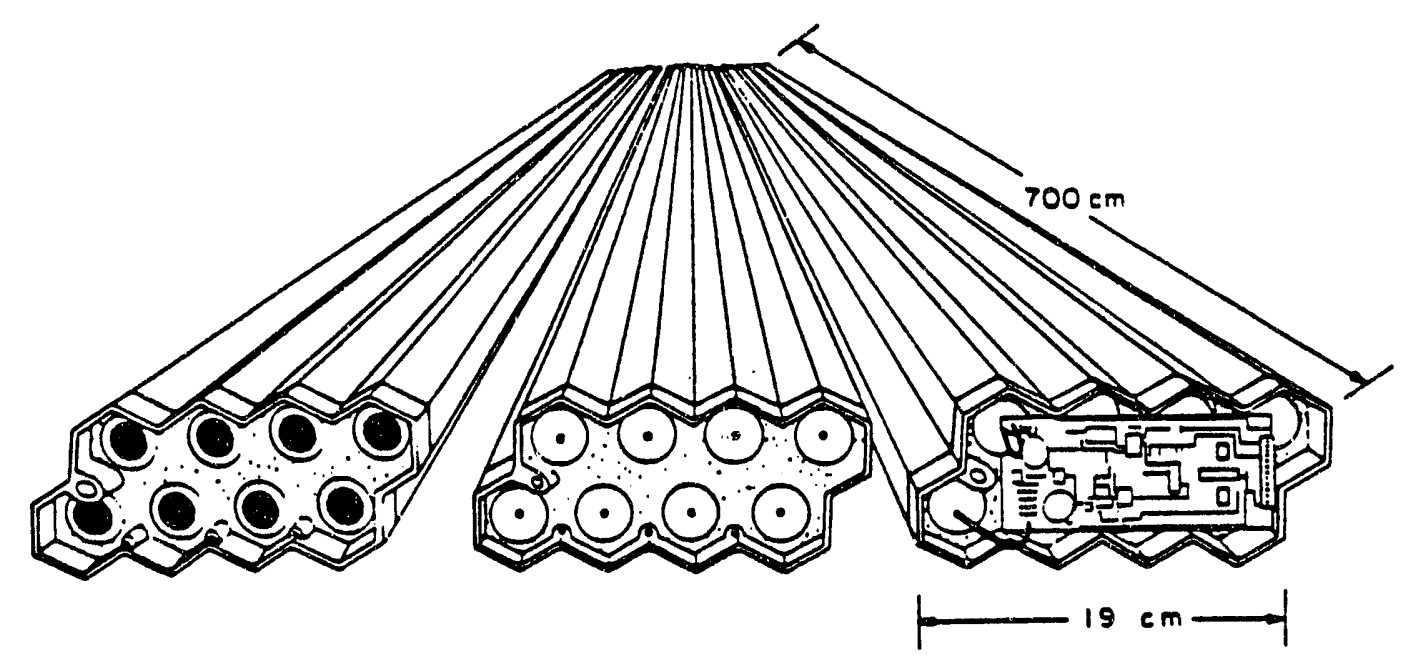

Figure 2.5: End view of three adjacent veto shield manifolds.

detector is called the 'in-layer', while the other one is the 'out-layer'.

The width of a veto shield manifold layer is $18.7 \mathrm{~cm}$. The muon tracking resolution is poor compared to the central detector; it is about $30 \mathrm{~cm}$ (one and a half manifold widths) for coordinates transverse to the manifolds and degrades rapidly (to $\approx 7$ meters) for orientations which are more parallel to the manifolds within a shield panel. The total area of coverage is around $1700 \mathrm{~m}^{2}$; the surface area of the ceiling is $420 \mathrm{~m}^{2}$. Smaller panels, called gap fillers, have been added to cover the access areas between the larger panels. Unfortunately, gap fillers augmenting the veto shield floor panels were not in place when the data was taken for the analysis reported here. There was a large gap in the northern floor section, and the north wall had not been deployed. Although some of gap fillers in the vertical wall section (east, west and south) were actually in place, we did not utilize them in the present analysis. In order to maintain simplicity in programming for data processing and analysis, every shield panel was assigned to a 'superpanel'. Thus, all of the ceiling forms superpanel \# 1, and all of the floor panels were assigned to superpanel \#2. 
All east wall panels, all west wall panels, and all south wall panels were assigned to superpanels \# 3,4, and 5, respectively. The north wall was introduced into the data stream in the spring of 1993.

\subsection{Electronics for the Veto Shield}

When a charged particle passes through the gas within a shield manifold, a voltage drop is created along the sense wire due to jonization which initiates a charge avalanche into the wire. This signal is amplifie $d$, converted into a digital signal by the Digital Output Card (section 2.4.1), and then read out by a Digital Readout Module (DRM - see section 2.4.2). Each DRM is mounted into a CAMAC crate. The response time (signal collection time) of a shield module is slightly less than $1 \mu \mathrm{s}$, and thus reasonably matched to the central detector's time resolution. The activity of the shield is sampled in $1 \mu \mathrm{s}$ bins. The information is stored until a main detector trigger occurs. When the main detector trigger arrives at a DRM, its stored information is read out.

\subsubsection{The Digital Output Card}

Each front-end electronics card contains two channels which service upper and lower layers of a veto shield p-tube manifold. A circuit for each channel (Fig. 2.6) performs three functions: (i) It amplifies the small current pulse arriving from the sense wires and converts it into a voltage pulse with a gain of $190 \mathrm{mV} / \mu \mathrm{A}$ [24]; (ii) amplified signals are then discriminated with a threshold level set at $100 \mathrm{mV}$ at the comparator; (iii) signals above threshold are transmitted through the multivibrator which generates a digital pulse, whose length of $1.2 \mu \mathrm{s}$ is set by an RC circuit. The output of the multivibrator is sent through a line driver which drives the pulse along a twisted wire pair to a DRM, where it is saved for future readout. We note that in this design, only the timing of a veto shield signal is measured; no information 


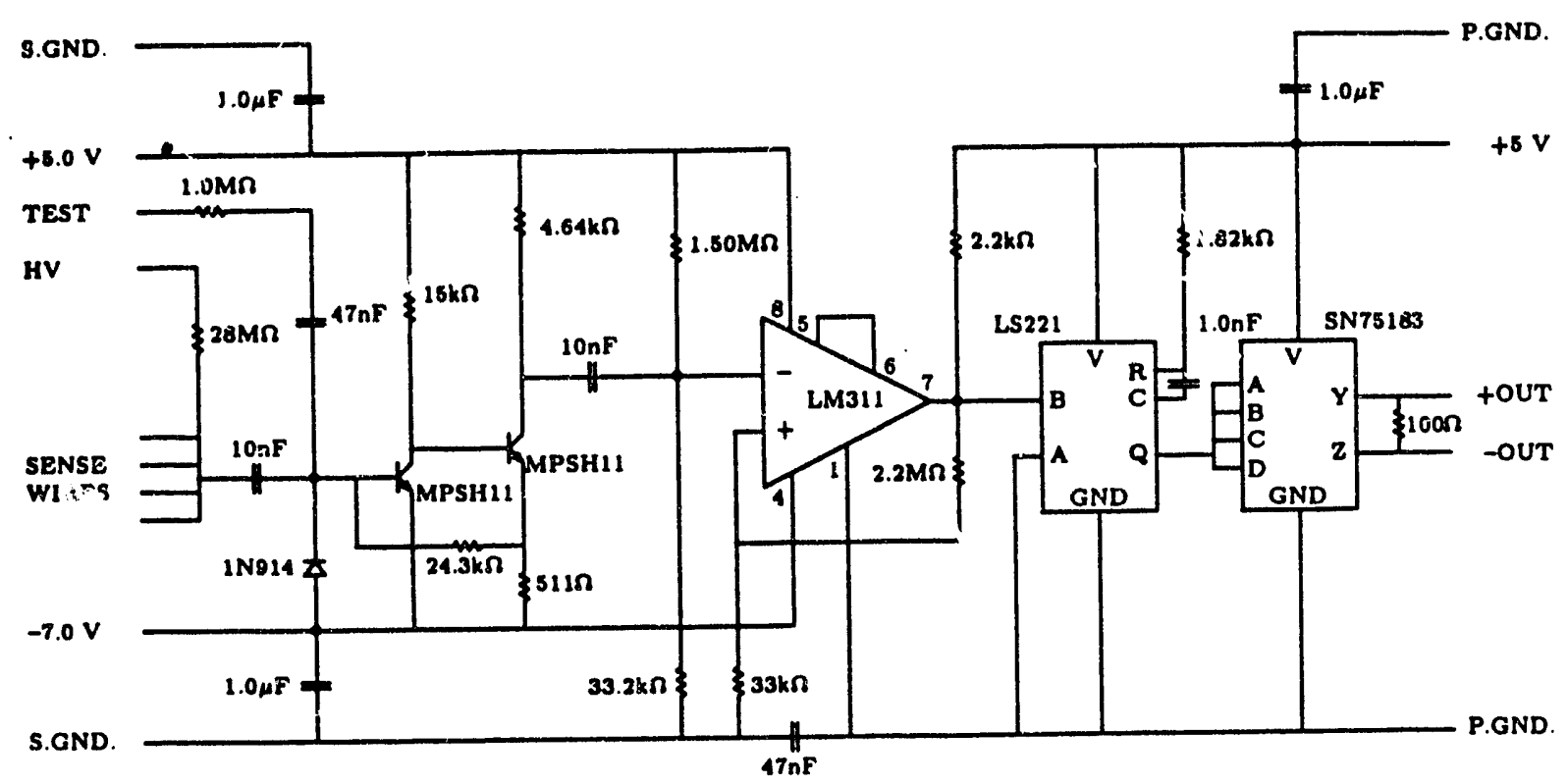

Figure 2.6: J̄cher:atic diagram of a digital output card.

on pulse height is available. The output card components are mounted on a $6 \times 14$ $\mathrm{cm}^{2}$ printed circuit board. Great care was taken in the design to avoid cross-talk between the two amplifier circuits. The amplifier components were separated on the board as much as poisible, and two separate grous is, namely the signal ground and the power ground, have been used. The two grounds are connected through a coupling capacitor on the circuit board. The only place where the power ground and signal ground are directly connected is at the control section, which is far from the circuit board. The resistor and capacitor values were chosen to avoid possible oscillations in the signal, particularly within the comparator circuit.

\subsubsection{Digital Readout Module}

The DRM was designed 'oy W. Oliver at Tufts University [25]. This is a single width CAMAC module which consists of six layers of printed circuits. It provides 64 channels to service 32 veto shield manifolds. Ea DRM accepts a central detector 
trigger and generates a ready-for-readout signal. The data sampling rate is $1 \mathrm{MHz}$. A DRM also forms veto shield coincidences $1.2+2.3+3.4+\ldots \ldots+62.63+63.64$ which were used in a precursor experiment [24], but not in our analysis. The signals from each channel of the veto shield are saved in 128 delay-lines for every $1 \mu$ s for future readout. The data scanning time is $1 \mathrm{~ms}$. When a trigger is received from the central detector, the previous $128 \mu$ s history of shield hits is read out.

\subsection{The HPW Enhancement Array}

To use the veto shield as a stand-alone muon detector, all three spatial coordinates for through-going muons should be measured. In the existing shield, depending upon the tube orientation in the individual panel, the third spatial coordinate can he obtained in limited, specific cases. For example, if there is a through-going muon which is incident from the ceiling and exits through the west floor panels, the third coordinate can be calculated if only the direction of the muon is available. This situation will be discussed in Chapter 3.

If we have proportional chambers which are deployed in a criss-cross pattern, it is possible to have all spatial coordinates and to deduce the direction of throughgoing muons independently of the central detector. Proportional tubes which were acquired from the defunct Harvard-Purdue-Wisconsin (HPW) proton decay experiment have been already deployed on one portion of the east ceiling area. These tubes are laid transverse to the Tufts shield manifolds in the ceiling. Details concerning the HPW chambers and their electronics are given in Appendix D. In a fully deployed configuration, called the "super-shield", coarse X, Y, and Z coordinates for through-going muons will be provided which will yield an angular resolution of about $1.5^{\circ}$ [27]. Additional information on HPW chambers is given in references [28] and [29].

Currently there exist two HPW panels on the east ceiling, with 32 chambers in 
each panel; these panels have been operating for more than a year. However, the data from these initial HPW panels are not included in this analysis. To obtain the direction of a muon, we also need criss-crossed panels on the veto shield floor. In the future, two criss-crossed layers of proportional tubes will cover the entire ceiling, floor, and possibly all of the side walls, if the TASSO array is ever deployed. With these enhancements the shield will finally become a stand-alone muon telescope.

\subsection{Triggering}

Full details of the trigger system for the central detector are given in references [20] and [21]. A brief description of the triggering of an event is given below. As described previously, traversal of charged particles through drift tubes of the central detector results in ionization segments whose drift electrons create analog signals on anode wires and cathode strips. The analog signals are immediately amplified and digitized. The leading edge of a pulse above the central detector threshold is called an 'edge'. The trigger system requires a minimum number of 'edges' to trigger an evert. Specifically, seven 'edges' from any sixteen adjacent anodes OR eight 'edges' from any sixteen adjacent cathodes within a resolving time of $72 \mu$ s are required to trigger an event for subsequent read out. All 'edges' are separated by at least $0.6 \mu \mathrm{s}$ [13]. (Effects of such a trigger on detector acceptance are described in Section 4.6.)

Localized regions of ionization can arise near to the calorimeter wire plane as the result of Compton electrons scattered by photons from the cavern rock or from the calorimeter itself; these are called "blobs". The requirement of time separation among pulses avoid triggering due to 'blob' pulses which start at the same time. Usually a blob will give only one edge.

A typical through-going muon track (see Fig. 2.7) [31] will easily satisfy the above trigger condition. An energetic muon track, either in a single or multiple muon event, almost always produces more than seven edges with enough time separation to 


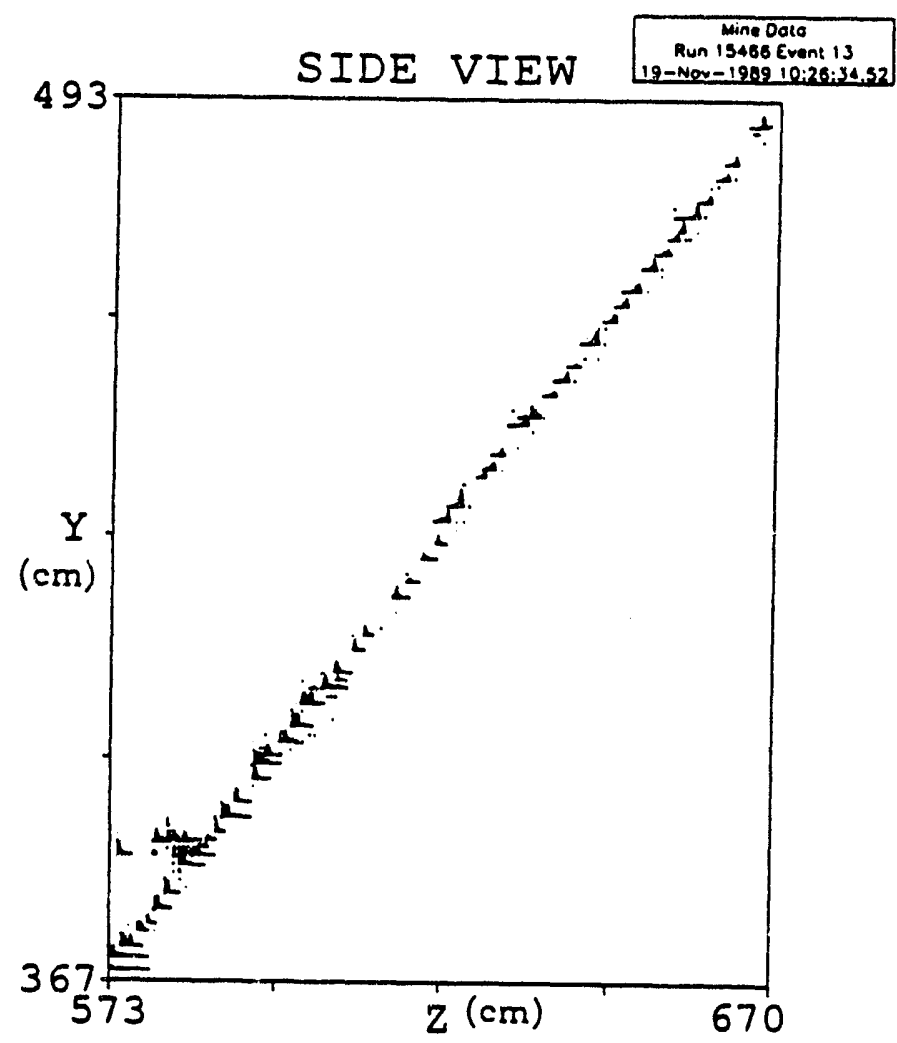

Figure 2.7: A through-going single muon track with a small knock-on electron trigger the system. However, if a muon comes in exactly from the vertical direction, then the time separation between edges is nearly zero. In this case, a vertical muon will not trigger the system and the event is lost. (See Fig. 4.2 for the effect of trigger condition in the zenith angle distribution.)

Since our main analysis is based upon the multiple muon events, we have to implement off-line software code to identtify the multiple muon events among all of the trigger events. The off-line codes which were used to separate the trigger events into different categories, and to reconstruct multiple muon events using veto shield as well as central detector information, are described in the following sections and in Chapter 3. 


\subsection{Software and Data Processing}

Detailed information on Soudan 2 on-line software, on the readout sequence for data acquisition, and on off-line software for dat processing, can be found in references [20] and [21]. Here we briefly describe the aspects of the off-line software which have been utilized for our multiple muon analysis.

Every event that satisfies the central detector triggering requirements is written out for further processing. A collection of events representing a pre-determined amount of data (about 2100 events in 1991) is called a 'run' and is assigned a fivedigit identification number. All the basic off-line data processing is performed by the Soudan Off-line Analysis Program SOAP at the Soudan site. As configured for this purpose, SOAP processes and reconstructs each event and identifies candidate muon tracks (stopping or through-going, single or multiple), candidate contained and partially-contained events, and candidate magnetic monopole tracks. Starting in 1991, events of different types were separated into different data files.

In SOAP, all Soudan 2 data are stored in a one-dimensional array organized into data 'banks' by the BOS dynarnic memory management system. SOAP represents an overall framework within which individual tasks are performed by software modules called 'processors'. Processors performing basic functions are PGMINI to initialize the program, PGMEND which is called at the end of a computer job, EVREAD which handles event input, and EVWRIT for event output. Central detector data are handled by CREPLR (unpacks raw data), SOFTPL (generates clean 'software' pulses), PMT (matches anode and cathode pulses to find the XY projection of a central detector hit), RUFT0 (estimates $T_{0}$ - see Section 2.8), SEARCH (finds straight lines in the $\mathrm{XZ}$ and $\mathrm{YZ}$ projections), and TRAJEC (finds straight lines in three dimensions - not used in our analysis). SPLASH is the processor that handles veto shield data (see below). Among processors that search for events of particular physics interest we use only MMU, a processor written to find multiple muon events. 
The Soudan 2 collaboration has developed its own database system. The necessary software, written by $\mathrm{N}$. West (Oxford), allows easy storage and retrieval of detailed time-dependent information about the detector as it is being developed and on the data as they are being acquired. The detector constants and any constants needed by the individual SOAP processors are stored in 'titles' banks managed by the database system.

The Subroutine to Process and Locate Associated Shield Hits, or 'SPLASH', performs all the basic handling of the veto shield data:

(i) SPLASH unpacks the raw shield data. The form of the raw data is determined by the design of the Digital Readout Modules (Section 2.4.2) and by the CAMAC IO-list [26]. Every electronic channel of the veto shield is identified by the CAMAC crate number (1-5) and CAMAC-crate slot (or DRM) number (1-15), and DRM channel number (1-64). The crate number and the slot number are transmitted directly by the IO-list. The DRM channel number is packed in the actual data words together with timing information. SPLASH performs extensive checks of the raw data structure, marks any error conditions found, and unpacks the raw data when no errors were found. The output is stored in 'SHLT' data banks which carry the veto shield time slot (1-128) as their bank number, and each data word contains the crate number, slot number, and DRM-channel number packed into 16 bits.

(ii) SPLASH provides information on hardware performance to identify malfunctioning shield elements during data acquisition. Information on detector faults is also compacted for later inclusion into the Soudan-2 database.

(iii) SPLASH translates the veto shield information from "crate-slot-DRMchannel" into "panel-tube-layer". The mapping between the readout information and the actual panel numbers is performed using Veto shield Database bank 1, VDB1, stored in the Soudan 2 database.

(iv) SPLASH performs pattern recognition. It scrutinizes the veto shield hit pattern to identify groups of hits that occur within a narrow time window $(1 \mu \mathrm{s})$ and 
are contiguous in space. These are called 'adjacent (shield hit) groups' throughout this Thesis. (More on veto shield hit configurations in Section 3.1.)

(v) SPLASH performs geometric reconstruction. Using information from the veto shiuld Database bank 2, VDB2, SPLASH finds the position in space for all identified adjacent groups, or for any shield hits needed for the analysis.

Every SOAP execution can be tailored using commands in a ASCII text input stream to select processors, to specify input and output files, to select events, to modify title banks, etc. Additional title banks can also be supplied in the same input stream either to supplement or to override the database information.

The SOAP routines are organized into libraries, updated and released to the collaboration about twice a year. Release-13 (Release-15) SOAP was used for processing our 1990 (1991) data. All of the 1990 data had to be processed at Tufts before our event selection, but only events passed by the MMU processor had to be handled in the 1991 data. Events are identified by at least one of several software multiple muon trigger algorithms, as described in Section 3.2

\subsection{The Event Time; SEARCH Output}

The SEARCH processor, written by P.J. Litchfield (RAL), is a powerful pattern recognition tool for central detector data. It aims to identify hits that form a straight line in the anode-time (XZ) projection, and, independently, in the cathodetime (YZ) projection; then corresponding matching projection pairs are found. The SEARCH output used in our analysis is stored in ATLN ('Anode Time LiNe') and CTLN ('Cathode Time LiNe') data banks in the form of slopes and intercepts for all straight lines found.

Important for our event analysis is determination of $T_{0}$, the "event time". $T_{0}$ is the earliest time when a charged track crossed drift tubes within a module and deposited the initial ionization. This ionization is detected by a wire-plane at the end 
of the drift tube after a time determined by the drift distance (drift direction is along the $\mathrm{Z}$ axis) and drift velocity, $t_{\text {drift }}=Z / v_{\text {drift }}$. Only relative timing and relative $Z$ coordinates of signals from individual drift tubes within a central detector module are therefore measured, with one exception. When a track crosses a wire-plane, the drift time is zero, and the absolute time is known. Also, $T_{0}$ can be calculated when a track crosses the center of a module where the electric field changes its direction, i.e. ionization from one part of the track drifts toward one wire-plane of the module, and ionization from the rest of the track drifts toward the wire-plane at the opposite end of the module. In other cases, we can determine only a range of possible $T_{0}$ values whose extent depends on possible drifting distances, as estimated by processor RUFTO using matched anode and cathode pulses (i.e. pulses with X and $\mathrm{Y}$ coordinates known).

The SEARCH processor then tries to obtain the best $T_{0}$ value based on analysis of pulses which originated in the wire plane area, on straight-line fits for matched anode-time and cathode-time projections, and on information supplied by the veto shield. Anode-time, cathode-time and their error values have been used to determine the average $T_{0}$ for our muon reconstruction. Further details on $T_{0}$ and its relationship to the shield timeslot will be discussed in Chapter 3. 


\section{Chapter 3}

\section{Data Selection and Cuts}

\section{Introduction}

We have aeveloped a software code to reconstruct candidate muon tracks using the veto shield hit configurations. We rely solely upon the veto shield for our off-line selection of multiple muon events ("software trigger"). We have required that there be at least one central detector track which is coincident in time with associated shield hits. We define the direction of the muon bundles using the central detector $\operatorname{track}(s)$ and then use the veto shield to determine the bundle multiplicity. In Section 3.1 we describe the various kinds of shield hit configurations we have used. Data processing and initial event selection are described in Section 3.2. Sections 3.3 and 3.4 summarize how the selection of muon tracks has been made for the central detector and for the veto shield respectively. We introduce some initial physics observations with reconstructed multiple muon bundles in Section 3.5. Some representative muon bundle events are displayed in Section 3.6 in order to illustrate the different types of candidate muon tracks. Displays of this kind have served as an aid to visualizing the events in three dimensions and have been an essential tool for the carrying out of checks of our muon reconstruction algorithms.

\subsection{Shield Hit Configurations}

Candidate multiple muon events are selected based upon hit patterns observed in the veto shield. As described in Chapter 2, each veto shield proportional tube 


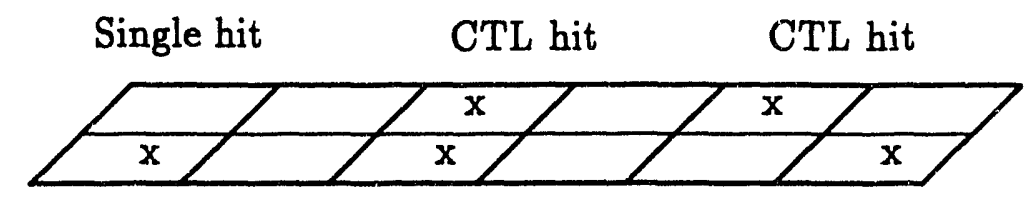

Figure 3.1: A single hit and Coincident-Two-Layer hits.

has two layers; each layer consists of four adjacent cells with sense wires connected in parallel. If ionization "lights up" any one of the four sense wires within one layer and all neighboring layers are quiescent, the pattern is called a 'single hit'. On the other hand, if cells from two neighboring layers light up and the cells form an in-out combination, the configuration is called a 'coincident two layer hit' (CTL). Note that the two hits of a CTL can be within one veto shield module, or in neighboring modules occurring in the same or adjacent $1 \mu \mathrm{s}$ time slot. These two types of hit patterns are illustrated in Fig. 3.1

The frequency of occurrence of single hits in a typical seven - meter - long veto shield tube layer, is $300 \mathrm{~Hz}$ due to natural radioactivity present in the Soudan 2 cavern. The singles rate in the entire veto shield is about $0.8 \mathrm{MHz}$. The rate of occurrence of CTL's is $3 \mathrm{~Hz}$ per manifold, or $4 \mathrm{kHz}$ for the whole shield; most of these are also believed to be caused by local radioactivity. The accidental rate for CTL hits in our shield manifolds, which have a $1 \mu \mathrm{sec}$ time resolution, is sufficiently low to ensure that such hits provide a reliable tag for through - going muons. Additionally, single hits can reasonably be utilized when coincident with central detector data, as considered in Section 3.4 below.

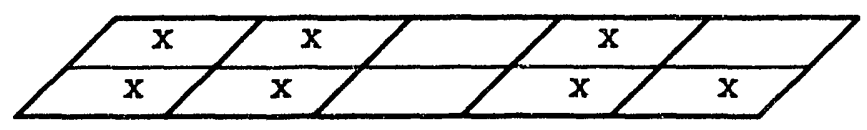

Figure 3.2: Adjacent Groups. 
When an energetic muon travels through matter, it may initiate knock-on electrons ( $\delta$-rays). When a muon is accompanied by such a self-induced electromagnetic shower, there may arise, in addition to the two-layer coincident hits due to the muon itself, hits in nearby modules due to $\delta$-rays. We therefore consider shield-hit topologies which are larger than a CTL to be signalling the passage of a single muon, just as we ascribe a single CTL to a single muon. Three such topologies are as follows:

(i) A configuration of any number of (overlapping) CTL hits which occur in adjacent tube-layers, having no spatial gaps, in any particular $1 \mu$ s time slot window, is called an adjacent-hit group. Fig. 3.2 shows an example of two distinct adjacent-hit groups.

(ii) Close adjacent-hit groups with small gaps between them are also regarded to be associated with each other (and with the same muon track) and are defined to be splat hits or splats. For most shield surfaces, two adjacent-hit groups are assigned to the same splat if the shield manifolds involved are within $24 \mathrm{~cm}$ of each other. An exception is made for hit associations between east and west ceiling panels, since these panels overlap along the north-south centerline but with an elevation difference of $50 \mathrm{~cm}$. For hit associations between these panels adjacent-hit groups which are within $80 \mathrm{~cm}$ (3D separation) are regarded to be within a common splat.

(iii) Single hits which are coincident with adjacent-hit groups within a $3 \mu \mathrm{s}$ window and which are more than $24 \mathrm{~cm}$ away from a splat within a panel (coincident single - layer hits), are also retained for further consideration in the reconstruction of multiple muon events.

The shield time window extends from 1 to $128 \mu \mathrm{s}$. Hits within the earliest 20 $\mu$ s cannot be associated with a genuine multiple muon event trigger and so are eliminated from the set of hits analyzed in the event. 


\subsection{Data Processing and Initial Event Selection}

All event selections for our initial data sample were based on the number of adjacent-hit groups in the veto shield. For each Soudan 2 event, a 'Veto Shield Type', VS-type, is determined. First, every $1 \mu$ s time slot is considered separately. Events with no adjacent-hit groups have a VS-type of 0 in that time slot. The VStype is 1 if all adjacent-hit groups occur in the same super panel, a topology that is regarded to be a 'candidate partially contained event'. If an event has exactly two adjacent-hit groups in different super panels, the event is considered a 'candidate single through-going muon'. If both of these super panels are vertical, it is 'candidate horizontal muon' and its VS-type is set to 2 . VS-type $=3$ flags a hit topology with three or more than three adjacent-hit groups located in at least two super panels, considered to be 'candidate multiple muon events'. After the veto-shield type is determined for every time slot, the largest of all VS-types for the individual time slots is assigned to be the VS-type of the event.

The actual event selection is done by the SEARCH processor and MMU processor incorporating severwi unultiple muon algorithms, as the collaboration's intent has been to approach the analysis in several different ways, each with its own characteristic (and, hopefully, complementary) systematic errors. Two algorithms developed at the University of Minnesota rely on the central detector to determine the muon multiplicity; one counts tracks recrsnstructed by the SEARCH processor, the other examines the topology of reconstructed points not lying on a SEARCH-track to see if they might form a straight line. A variation of the first algorithm also looks among veto shield CTL's for a topology consistent with muon tracks parallel to an existing central detector track. The approach chosen at Tufts is based solely on the active shield, and - at this 'software trigger' level - does not rely on track reconstruction within the central detector. Within the MMU processor we require only that the candidate multiple muon events have VS-type of 3 , and that more than 3 adjacenthit groups occur in the veto shield. The MMU processor generates a logical OR of 
all the algorithms to select the candidate multiple muon events for inclusion in an 'MMU file'.

Two different data sets, one obtained July-August 1990 and a second set obtained during April through July 1991, have been used for the analysis of underground muon bundles presented here. For the 1990 data, the MMU files were not available, and all raw data tapes were processed at Tufts to select VS-type 3 events and to reconstruct central detector tracks in the events selected. Starting in 1991, the output of the MMU processor is created during the routine processing at the Soudan 2 site, and no further selection outside of the MMU files was needed at Tufts.

The daily rates for the candidate multiple muon events selected by our software trigger are shown in Fig. 3.3. The horizontal axis gives the date, month and year, while the number of multiple muon triggers for each day is shown on the vertical axis. After subtraction of 261.0 (52.8) hours of dead time, the total detector live time for the 1990 (1991) data sample of this study is 677.8 (1494.4) hours.

A total of $141,073(133,608)$ VS-type 3 events were obtained from the 1990 (1991) data sample. Before further analysis, we eliminate events which do not have any central detector track with associated veto shield hits, and also events which have more than 100 adjacent groups in the shield (impossible to reconstruct reliably). A total of 236,479 single (in central detector only) and multiple muon events (central detector and veto shield) remained. The event counts are summarized in Table 3.1.

\subsection{Selection of Muon Tracks in the Central Detector}

All candidate multiple muon events selected by the veto shield software trigger were scrutinized for the presence of through-going muon tracks in the central detector as determined by the SEARCH processor. The veto shield data were then examined for correlation with central detector tracks, both in time and in space.

The central detector - veto shield correlation in time can be described as follows. 

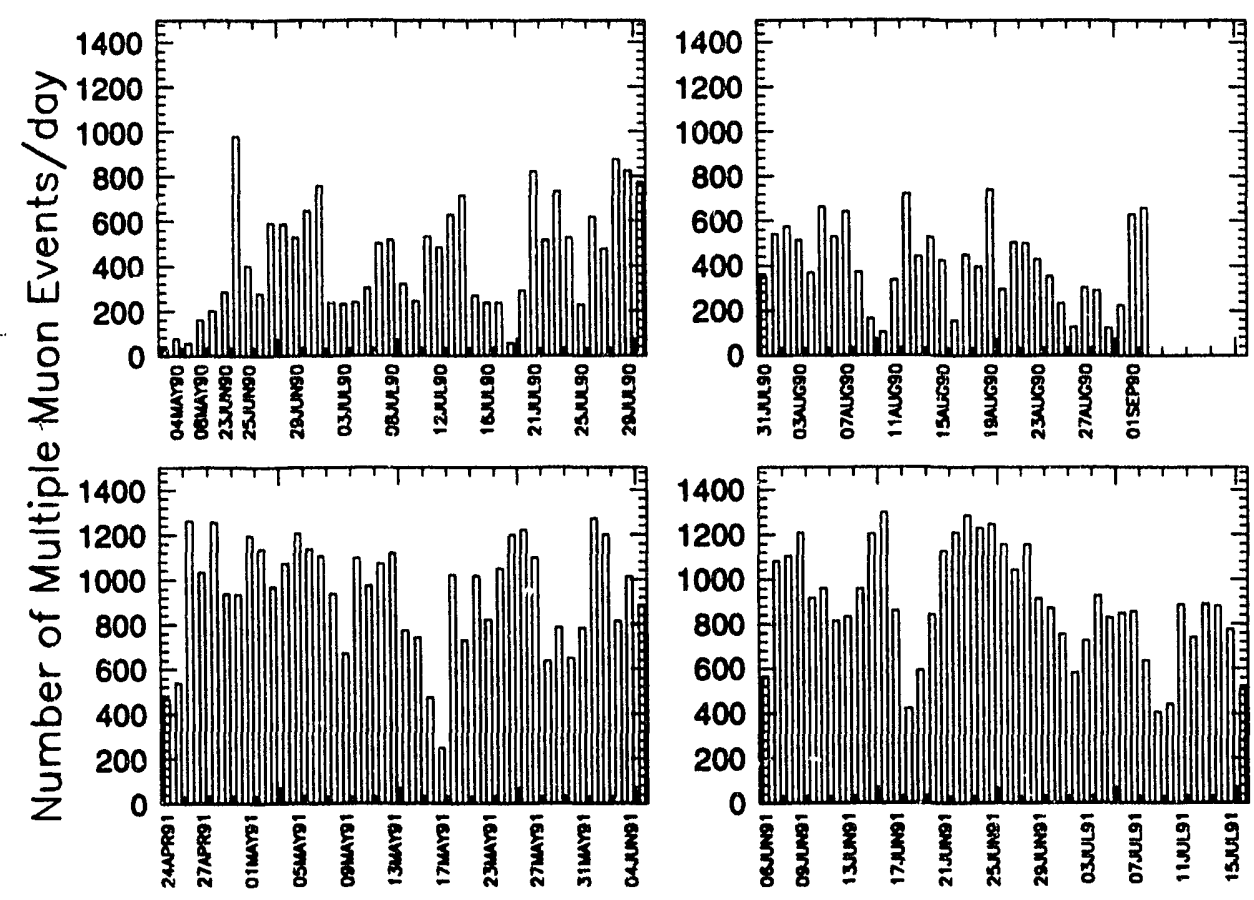

Figure 3.3: Multiple muon events per day.

The sequence of happenings starts with the muon depositing ionization in a veto shield proportional tube. A digital pulse results which is immediately inserted into a $128 \mu$ s long FIFO (First In, First Out) buffer. Next, the muon, when passing through the central detector, deposits ionization that drifts for up to $80 \mu$ s to a wire plane. When the subsequent voltage drop is recognized, a trigger signal is formed, transmitted to all veto shield CAMAC crates, and the information on the shield activity within the last $128 \mu \mathrm{s}$ is read out along with information on the central detector. The veto shield $1 \mu \mathrm{s}$ 'time slots' are numbered from 1 to 128 , with 128 $\mu \mathrm{s}$ being closest to the trigger time. The task is now to find the time of passage of the track through the central detector, $T_{0}$, in terms of veto shield time slots, $T_{C D}$. In the central detector, $T_{0}$ is measured in 'clock ticks', with 1 tick equal to 2 ns. If 
Table 3.1: Run Statistics for the 1990 an 1991 data samples

\begin{tabular}{|l|r|r|}
\hline \hline \multicolumn{1}{|c|}{ Event Selection } & \multicolumn{1}{c|}{1990} & \multicolumn{1}{c|}{1991} \\
\hline Total number of triggers & $1,299,349$ & $2,784,176$ \\
Multiple muon software trigger & 141,073 & 133,608 \\
Failure events due insufficient memory assigned by SOAP & 120 & 158 \\
Events having no tracks in the central detector & 31,781 & 6,261 \\
Eve.sts with one or more tracks in the central detector & 109,292 & 127,350 \\
Events with number of groups > 100 & 30 & 133 \\
Events with groups $\leq 100$ and with & & \\
at least one track in the CD & 109,262 & 127,217 \\
\hline \hline
\end{tabular}

$T_{\text {trig }}(=i 12$ ticks) is the time when the trigger is sent to the shield, we find [25]:

$$
T_{C D}=\left(T_{0}-T_{t r i g}\right) \times 0.2+t_{w}+t_{d}
$$

where $t_{w}(=128 \mu \mathrm{s})$ is the length of the shield time window, and $t_{d}(=1.5 \mu \mathrm{s})$ is the delay time between the sending of the main detector trigger to the veto shield and the onset of readout of the shield data. The latter off-set constant has been determined empirically by scanning muon events. The central detector $T_{0}$ is obtained by taking the average of the anode time $T_{0}^{A}$ and the cathode time $T_{0}^{C}$ under the following two constraints: (1) the difference between $T_{0}^{A}$ and $T_{0}^{C}$ should not exceed 100 ticks (200 ns), and (2) the errors on the $T_{0}^{A}$ and $T_{0}^{C}$ must be less than 30 ticks (60 ns).

Fig. 3.4 shows the distribution of $T_{C D}$, the time of a central detector trigger (Fig. 3.4(a)) and the leading-edge of a veto shield hit configuration, $T_{V S}$, (Fig. 3.4(b)) for individual events. The distributions are well-matched, indicating a very similar relationship to the actual event trigger time as expected. 


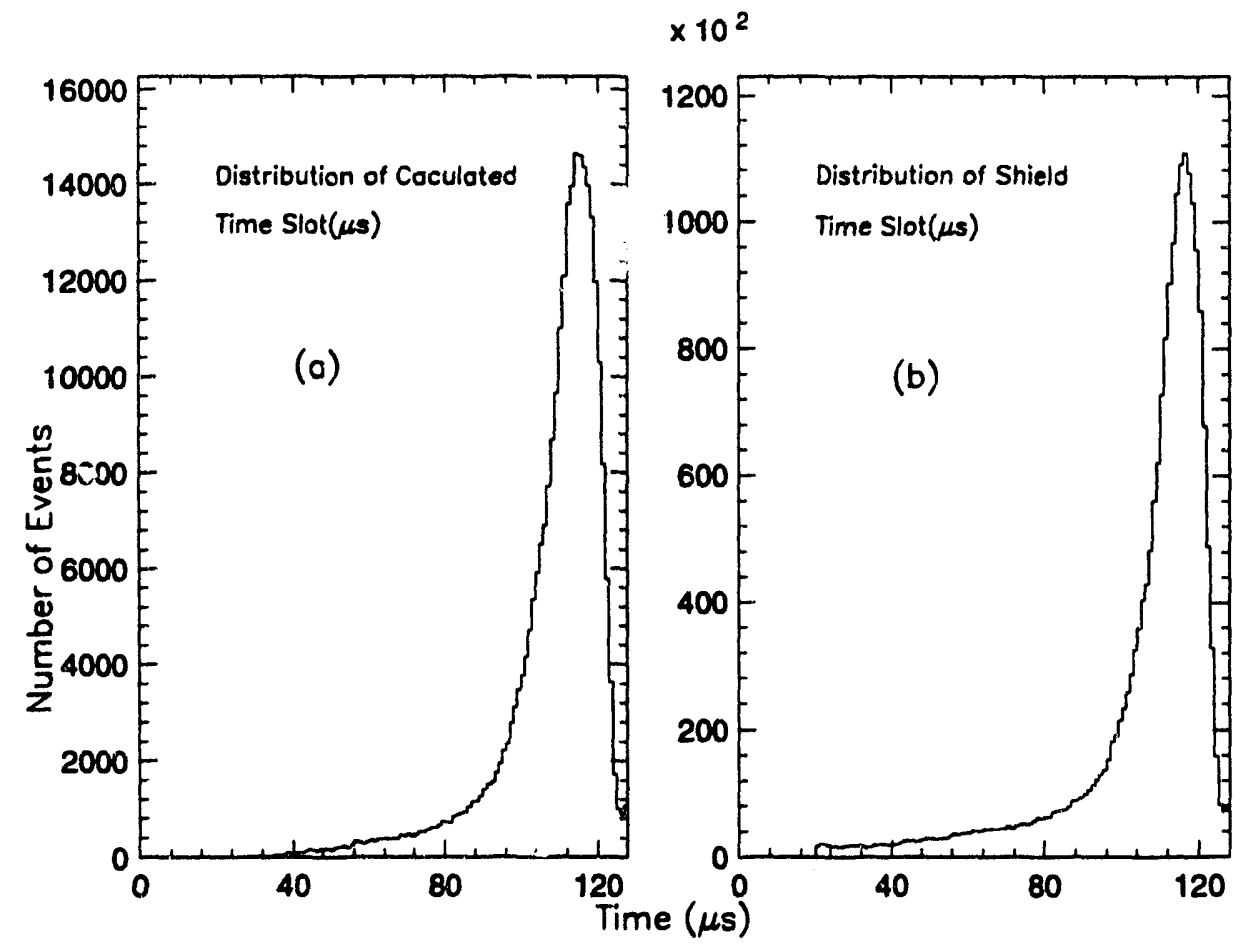

Figure 3.4: Distributions for the time of uccurence of muon events as estimated using (a) $T_{C D}$ and (b) $T_{V S}$.

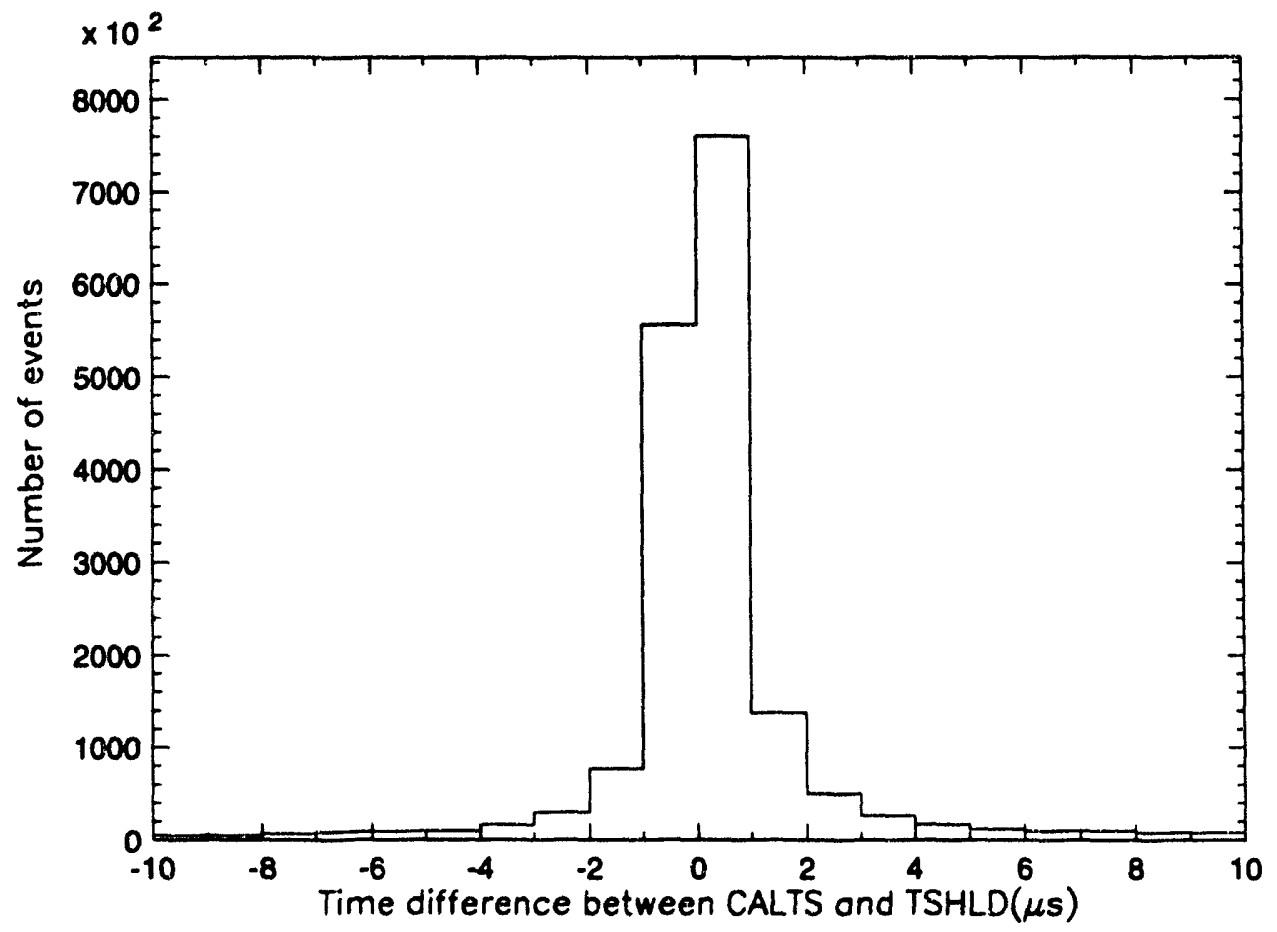

Figure 3.5: The distributior of the time difference between calculated time slot (CALTS) and shield time slot (TSHLD) $\left(T_{C D}-T_{V S}\right)$. 
Fig. 3.5 shows the distribution of the time difference, $T_{C D}-T_{V S}$, in $1 \mu \mathrm{s}$ bins. The distribution peaks at about $1 \mu$ s and is $3 \mu$ s wide.

In our analysis, we consider a central detector track to be correlated in time with a veto shield hit configuration, if the time difference between the leading-edge time slot for any adjacent-hit group and $T_{C D}$ is less than $10 \mu \mathrm{s}(3 \mu \mathrm{s})$ for 1990 (1991) data. Only single hits occurring within $3 \mu$ s of $T_{C D}$ are accepted for both data sets.

When considering the spatial relationship between central detector track and veto shield, we first obtain slopes and intercepts of the projections of matched tracks in the $\mathrm{X}-\mathrm{Z}$ and $\mathrm{Y}-\mathrm{Z}$ plane from the SEARCH processor output. We then calculate both exit points of each muon track in the central detector and extrapolate to the veto shield. Using the exit points, direction cosines for each individual track in the central detector are calculated. The direction angles are given by $\alpha$, the angle between the $\mathrm{X}$-axis (East-West) and the muon track, $\beta$, the angle between the $\mathrm{Y}$ axis (Vertical) and the track, and $\gamma$, the angle between the Z-axis (North-South) and the track. Fig. 3.6 shows the global coordinate system (GS) for the Soudan cavern.

To ascertain whether a central detector track is associated with an in-time adjacent-hit group or a single hit in the veto shield, coordinates of the shield exit points of the extrapolated muon track are compared to coordinates of adjacent groups or single hits. We should add that, due to the elongated geometry of the veto shield, the spatial correlations are well-determined for only one coordinate for each panel, namely the coordinate measured along a direction that is perpendicular to the veto shield manifold length. For example, if ceiling panels are considered, where the shield manifolds are placed along the $\mathrm{X}$ direction, it is the difference between the Z-coordinates of the extrapolated central detector tracks and a veto shield group or single that is calculated. If this difference is less than or equal to $40 \mathrm{~cm}$, a distance of about two manifold widths, then the group or single hit is considered as a hit associated with a central detector track. For all principal wall panels, the 

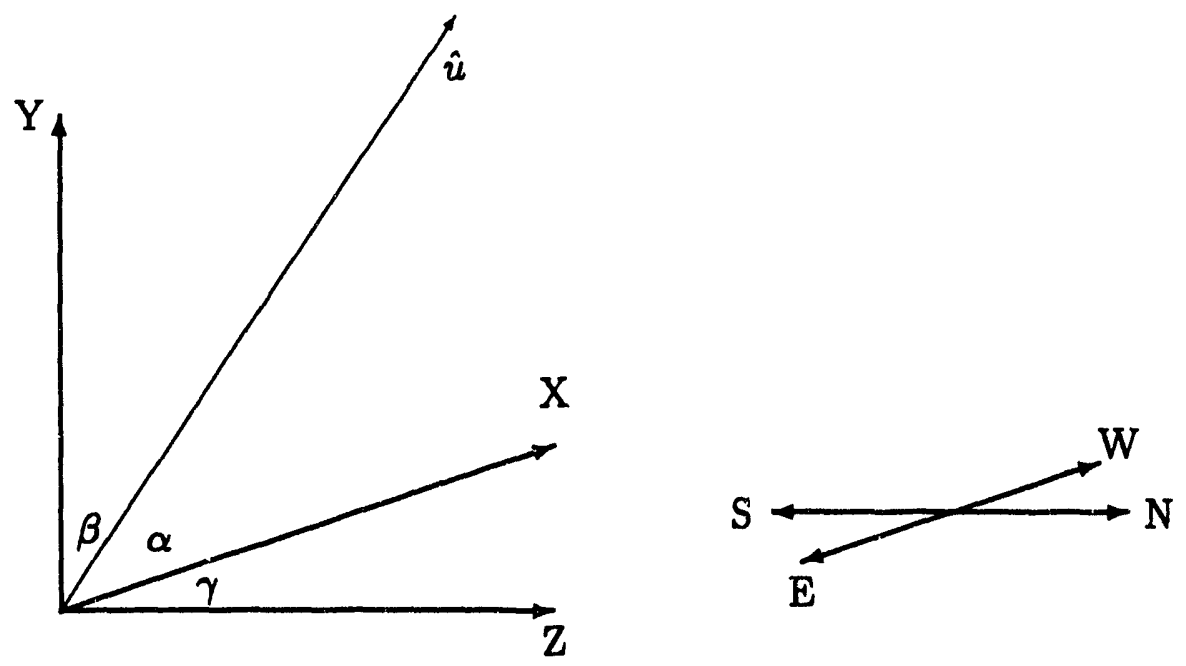

Figure 3.6: Coordinate system for the Soudan-2 cavern. Here $\hat{u}$ is the unit vector along a muon direction. $\mathrm{X}$ and $\mathrm{Z}$ axes are horizontal and the $\mathrm{Y}$ axis is vertical. $\alpha$ is the angle between $X$ axis and $\hat{u}, \beta$ is the angle between $Y$ axis and $\hat{u}$ (zenith angle) and $\gamma$ is the angle between $Z$ axis and $\hat{u}$.

Y-coordinates need to be used. For the east floor the Z-coordinates need to be used; for the west floor the $\mathrm{X}$-coordinates need to be used. The orientation of veto shield manifolds in various sections of the shield are shown in Fig. 3.7. Fig. 3.8 shows the distribution of the coordinate differences in $2 \mathrm{~cm}$ bins $(20 \mathrm{~cm}$ is the veto shield manifold width), compiled for the entire shield using the respective coordinate appropriate for each individual shield section. We find that most of the muon tracks in the central detector do indeed have associated shield hits.

Within the central detector, we define a muon "bundle" to be a group of muon tracks with an angle between two tracks not to exceed $3^{\circ}$ for all muon pairs. The distribution of angular separations between pairs of track in multiple muon events is shown in Fig. 3.9. In most of the events, the angle between two tracks is less than $3^{0}$. In candidate two-muon events where the angular separation is greater than $3^{\circ}$, the track which is more vertical is selected and is categorized as being a single muon in the central detector. 


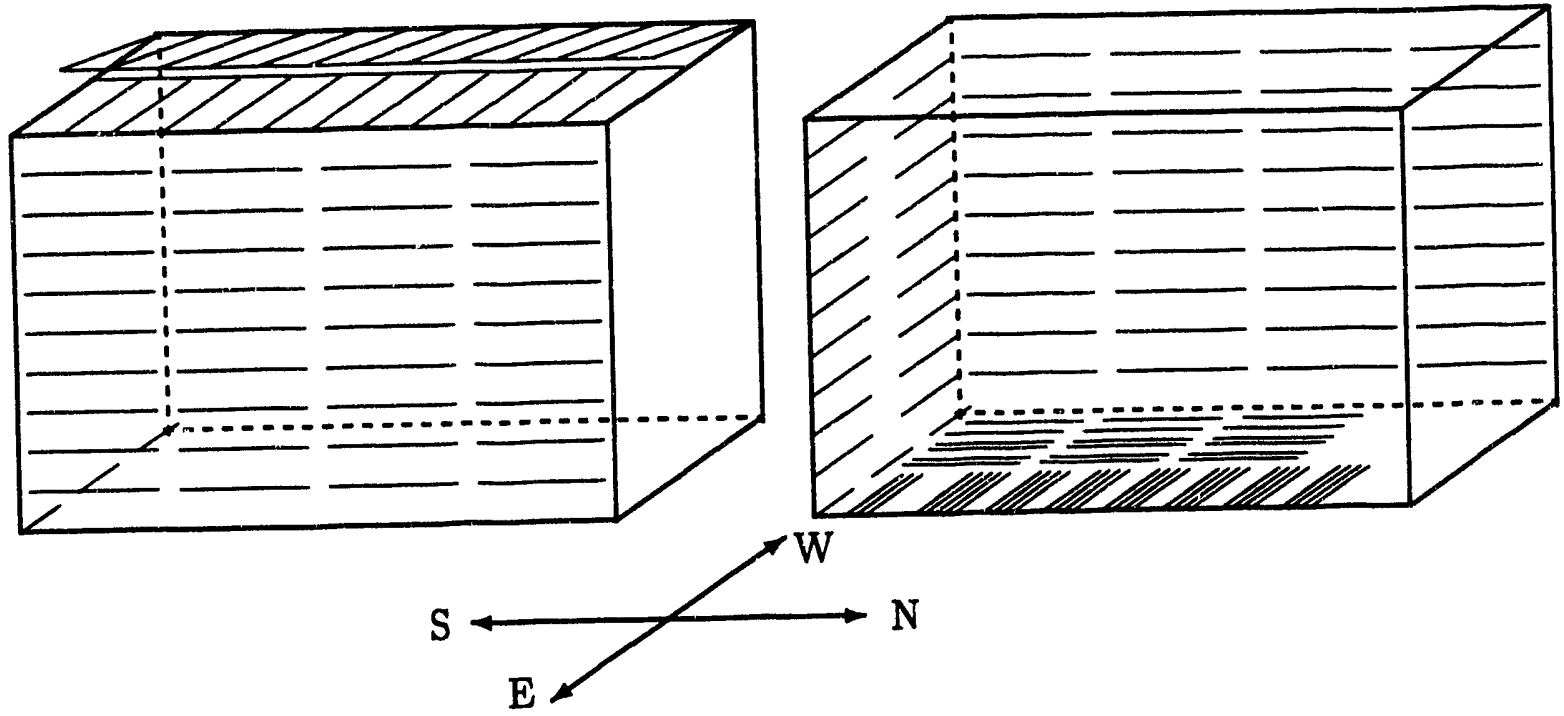

Figure 3.7: Orientation of proportional tube manifolds in the veto shield.

\subsection{Reconstruction of Muon Tracks Using the Shield}

To reconstruct a muon bundle using veto shield hits, we need from the central detector at least one track which defines the direction cosines of through-going muons. Consider the situation where there is one or more tracks in the central detector having associated shield hits. All shield hit groups or splats and singles associated with central detector tracks have been tagged, and so the remaining intime groups or splats and singles need to be considered in the reconstruction of additional tracks.

Track reconstruction using shield hits that are unassociated with CD tracks, is done by considering all reasonable combinations of panels. The veto shield manifold orientation for the various panels is taken into account when evaluating hit patterns for a candidate track. 


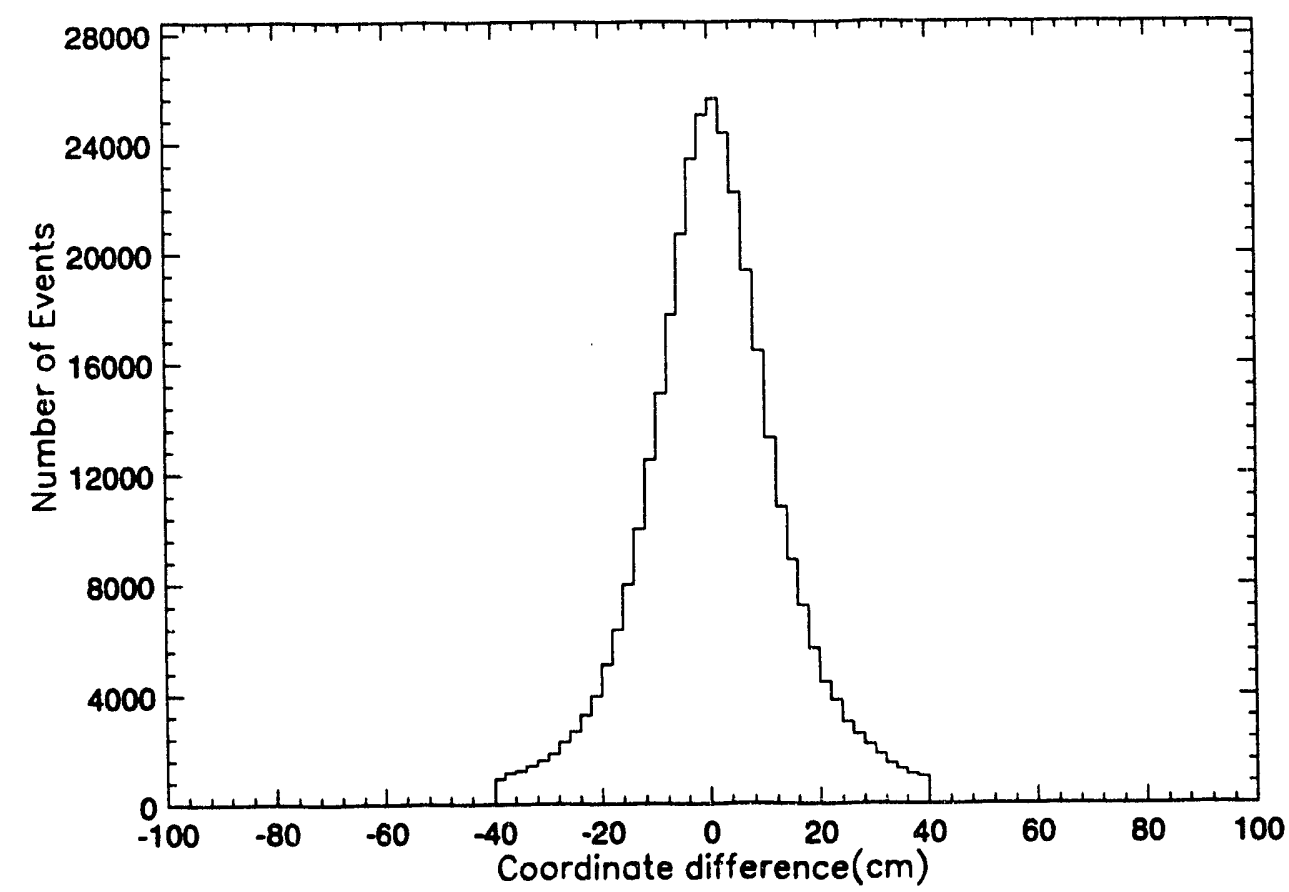

Figure 3.8: The distribution of coordinate differences shield hit and extrapolated central detector track, for all directions $\mathrm{X}, \mathrm{Y}$ and $\mathrm{Z}$.

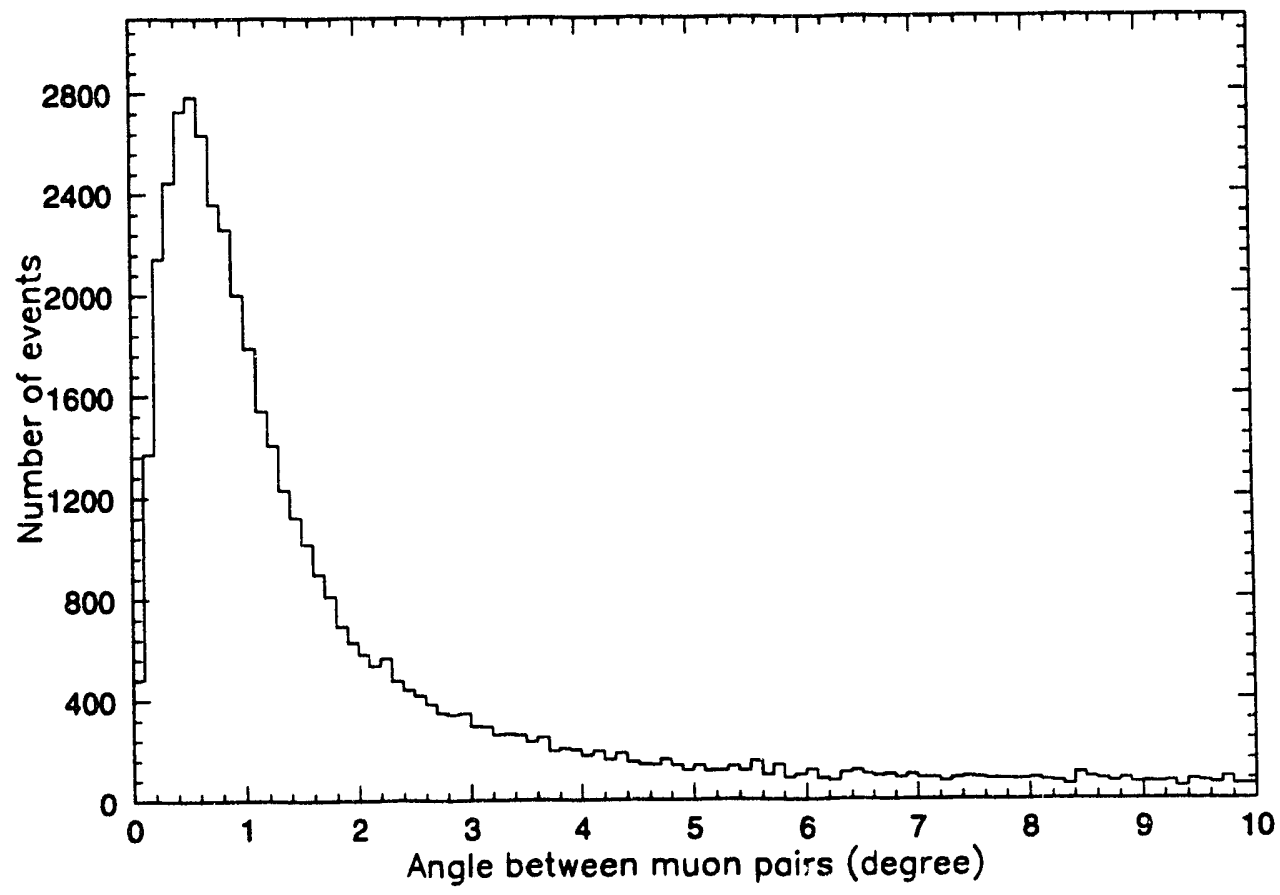

Figure 3.9: The distribution of angular separation between muon tracks within muon bundles, $\theta_{i j}$, angle between two muons within a pair. 
The following description is presented in terms of ceiling-west floor combinations, but is illustrative of all panel combinations. For ceiling panels, the shield manifolds run in the east-west direction; for west floor panels the manifolds are aligned in the north-south direction (see Fig. 3.7). For groups or single hits in the ceiling, the $\mathrm{Y}$ and $\mathrm{Z}$ coordinates are well-defined, but the $\mathrm{X}$ coordinates can vary over the manifold length of seven meters. For west floor hits, the $\mathrm{X}$ and $\mathrm{Y}$ coordinates are well-defined, but $\mathrm{Z}$ can vary along the manifold length. Fig. 3.10 shows a muon track and the shield manifolds it traverses in the west ceiling (WC) and in the west floor (WF). QR represents a manifold on the west ceiling, while PS is a manifold on the west floor which is oriented orthogonally to $Q R$ (as viewed from above, with the manifolds projected onto a horizontal plane). A muon trajectory is illustrated by the line $A B$. We denote the coordinates for the ceiling group by $\left(x_{1}^{1}, x_{1}^{2}\right), y_{1}$ and $z_{1}$, and similarly the coordinates for west floor hits by $x_{2}, y_{2}$ and $\left(z_{2}^{1}, z_{2}^{2}\right)$ where $x_{1}^{1}$ and $x_{1}^{2}$ are $X$ coordinates for the ends of the ceiling manifold, and $z_{2}^{1}$ and $z_{2}^{2}$ are $Z$ coordinates for either end of the west floor manifold. To get the entering point and exiting point of the track, coordinates $x_{1}$ for the ceiling hits and coordinates $z_{2}$ for the floor hits need to be determined. Differences between coordinates are related to the direction cosines and to differences between other coordinates according to the following equations:

$$
\frac{z_{1}-z_{2}}{\cos \gamma}=\frac{y_{1}-y_{2}}{\cos \beta}
$$

and

$$
\frac{x_{1}-x_{2}}{\cos \alpha}=\frac{y_{1}-y_{2}}{\cos \beta}
$$

From Eq. (3.2), the $z_{2}$ for the west floor panel can be determined; from Eq. (3.3) the $x_{1}$ for the ceiling panel can be determined. We refer to such coordinate combinations as " $M_{\perp}$ combinations", arising when manifolds are orthogonal in space (see Fig. 3.10). For the $M_{\perp}$ combinations, three coordinates for both entering and exiting points of a track can be determined. Errors for each coordinate can 


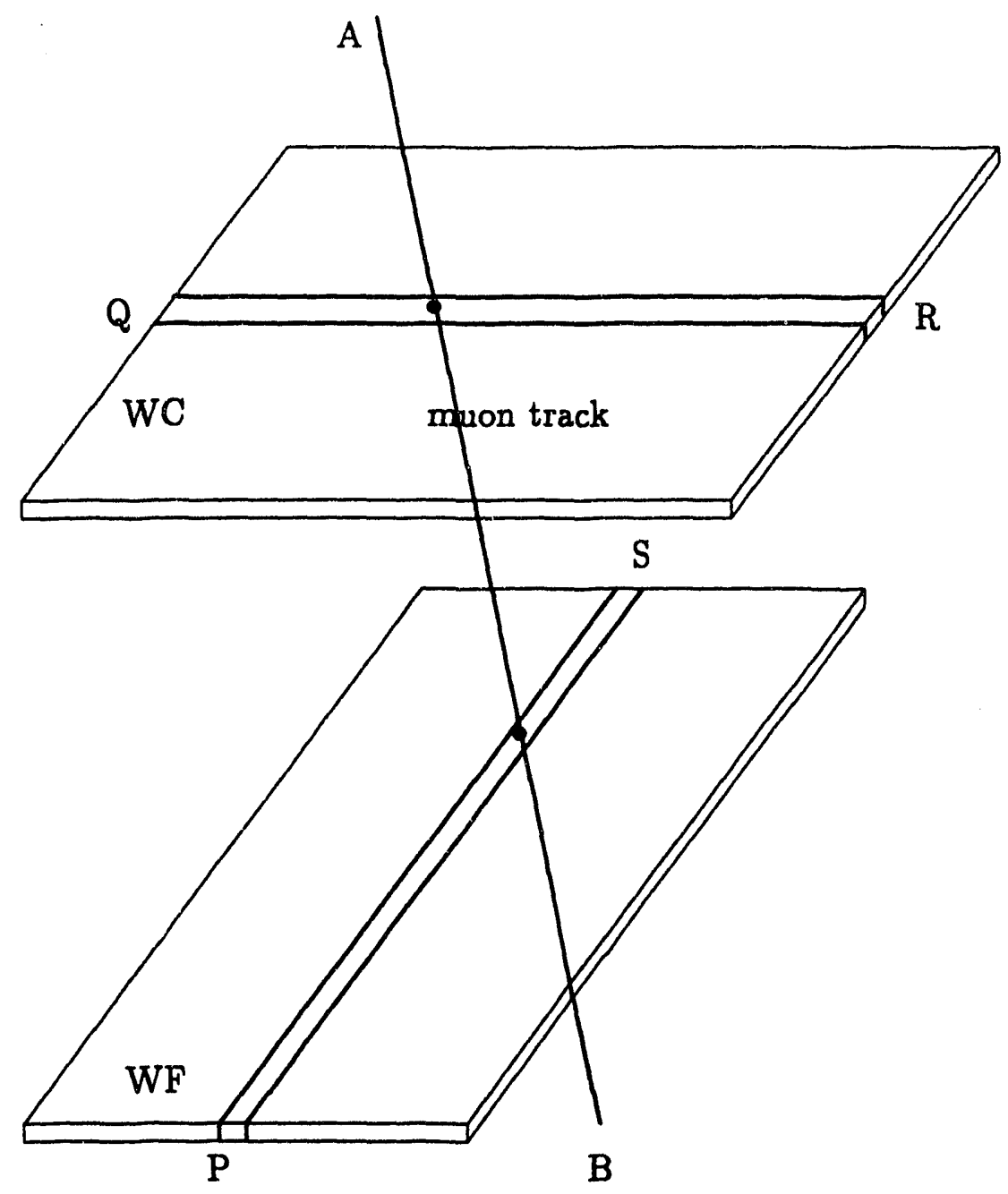

Figure 3.10: Muon track $\mathrm{AB}$ traversing an $M_{\perp}$ combination of veto shield manifolds. Manifolds QR and PS are orthogonal.

be calculated; the error assigned to direction cosines is calculated from the errors on slopes and intercepts of the track traversing the central detector. The error calculation will be discussed in detail in Chapter 4 .

A different situation arises when the ceiling panels (both east and west) are to be associated with east floor panels, for which the shield manifolds are also laid in the east-west direction (see Fig. 3.7). In this case, the $\mathrm{Y}$ and $\mathrm{Z}$ coordinates of candidate muons are well defined, however the $\mathrm{X}$-coordinates can be anywhere along a manifold length. Fig. 3.11 below shows how this case was handled to reconstruct a muon track from coincident shield hits on these panels.

For Fig. 3.11, two hit groups are recorded, one on the east ceiling (EC) and other on the east floor (EF). Two reference trajectories, QC and SD are constructed 


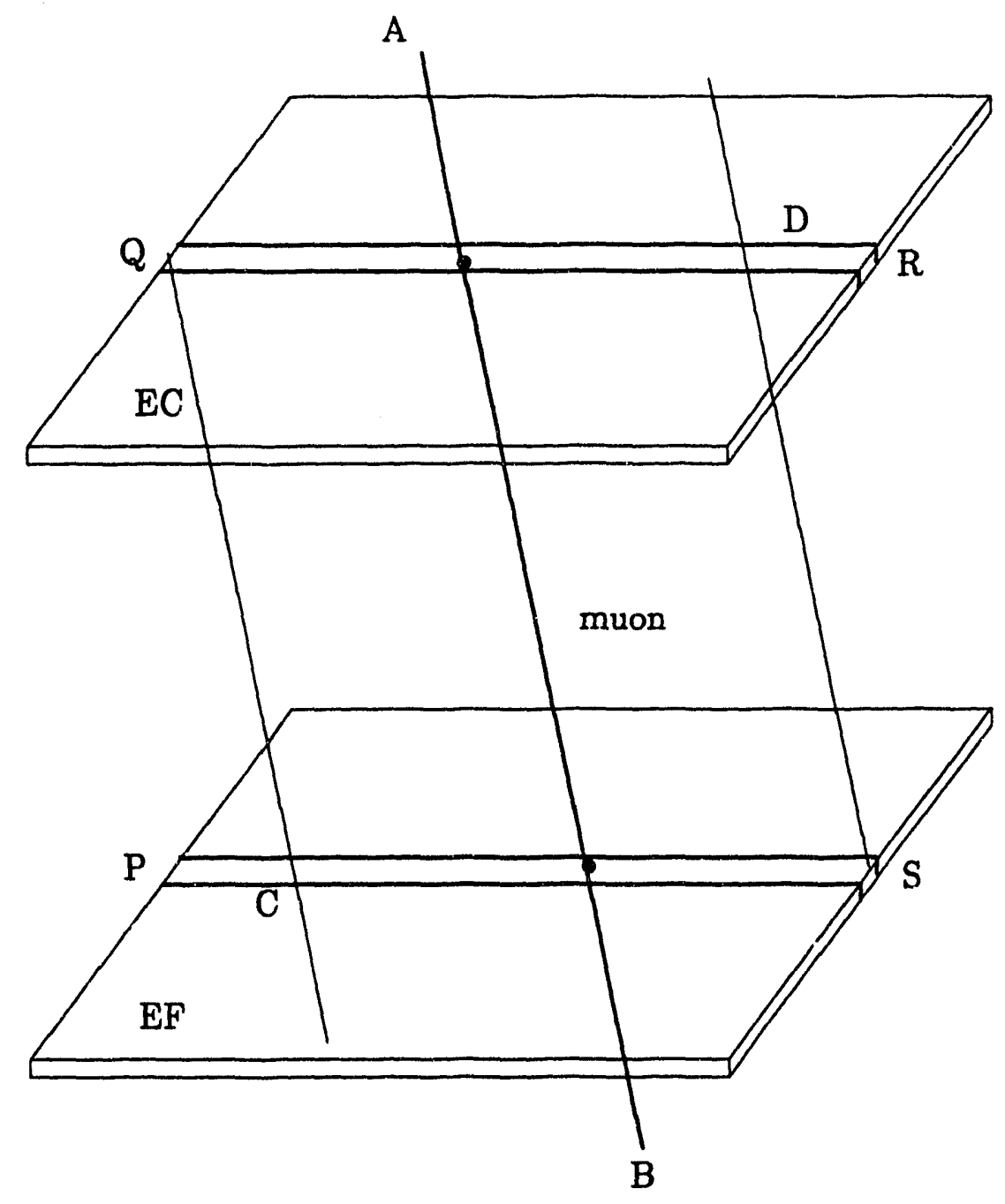

Figure 3.11: Muon track AB traversing an $M_{\|}$combination of veto shield manifolds. Manifolds QR and PS are parallel.

parallel to the central detector track. A muon track $A B$ is then reconstructed so as to be in the middle between QC and SD. The error for X-coordinates of the entering and exiting points of this track is taken to be half of the QD distance, i.e. \pm 3.5 meters (half of a VS manifold length), the error becomes smaller for tracks with larger zenith angle. VS combinations for which the traversed VS manifolds are parallel in space are called " $M_{\|}$combinations".

As illustrated in Fig. 3.7, the $M_{\|}$hit combinations are formed whenever throughgoing muons traverse ceiling and east floor panels, ceiling and south wall panels, east (west) wall and west floor panels or south wall and east floor panels. The $M_{\perp}$ combinations, on the other hand, arise whenever through-going muons pass through 


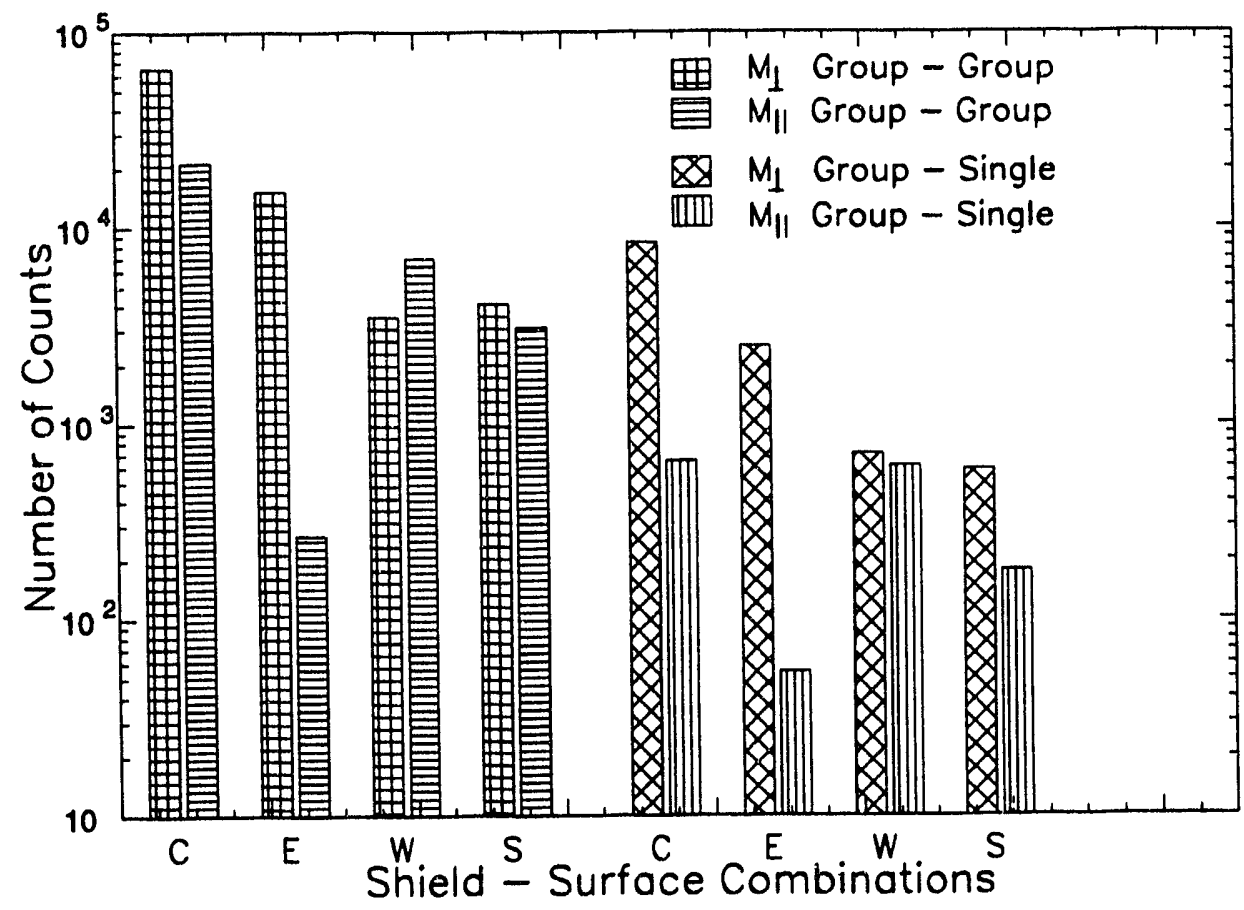

Figure 3.12: Number of $M_{\perp}$ and $M_{\|}$combinations for shield surfaces.

the ceiling and west floor, ceiling and east (west) wall, east (west) wall and south wall panels, or south wall and west floor panels. Fig. 3.12 shows the contribution of $M_{\perp}$ and $M_{\|}$tracks for group-group and group-single combinations, together with their association with various shield surfaces. Note that the statistics of the $M_{\perp}$ and $M_{\|}$tracks for different combinations are given by the logarithmic scale on the vertical axis, while the horizontal axis shows the various combinations of surfaces. The symbol $\mathrm{C}$ represents ceiling in combination with other panels such as floor, east, west and south walls, $\mathrm{E}$ is for east wall with floor and south wall, $\mathrm{W}$ is for west wall with floor and south wall and $\mathrm{S}$ is for south wall with floor. It is found that $M_{\perp}$ tracks constitute $75 \%$ of the total number of tracks. 


\subsection{Reconstructed Muon Bundles}

Candidate muon tracks which have trajectories reconstructed in the central detector together with associated veto shield hits, are called CLASS 1 candidate muons. If there are no shield hits associated with a central detector track, then the track is classified as a CLASS 3 candidate muon. Reconstructed shield candidate muons, including both $M_{\perp}$ and $M_{\|}$tracks, which are parallel to and coincident with central detector tracks, are categorized as CLASS 2 candidate muons. Splats on the ceiling which are not associated with any CLASS 1 and CLASS 2 candidate tracks and which are in time within $3 \mu \mathrm{s}$, are called "in-time isolated splats". Due to the existence of gaps on the floor during the period in which the data sample of this analysis was taken, we believe that these splats are associated with muon tracks which have passed through the floor gaps, or through the north wall area, which was at that time not instrumented. All in-time isolated splats on the ceiling are grouped into CLASS 4 candidate muons. An overview of our muon classification scheme is given in Table 3.2 .

Track lengths can be calculated for CLASS 1 and CLASS 2 candidate muons. For CLASS 1 muons, we require the track length within the central detector to be greater than $100 \mathrm{~cm}$. For CLASS 2 candidate muons, we require that the minimum length between shield surfaces traversed to be $200 \mathrm{~cm}$. The track length distributions are shown in the Fig. 3.13 and Fig. 3.14 for central detector tracks and for veto shield tracks, respectively. The length cuts remove all the "corner clippers". Two peaks are apparent in the distribution of track length for the central detector. The large peak reflects the vertical height of the central detector, that is most of the muon tracks are incident near to the vertical direction. The small peak reflects the width of the central detector along the east-west direction. Similarly, the large peak in Fig. 3.14 reflects the distance between a veto shield ceiling panel and a floor panel. 
Table 3.2: Muon classification for tracks within a muon bundle.

\begin{tabular}{|c|c|c|c|c|}
\hline \multirow{2}{*}{$\begin{array}{c}\text { CLASS } \\
\text { Number }\end{array}$} & \multirow{2}{*}{$\begin{array}{c}\text { Central } \\
\text { Detector Track }\end{array}$} & \multicolumn{4}{|c|}{ Veto Shield Hits } \\
\cline { 3 - 5 } & & Num. of Layers & Track End 1 & Track End 2 \\
\cline { 3 - 5 } 1 & \multirow{3}{*}{ Yes } & 4 & Group & Group \\
\cline { 3 - 5 } & & 3 & Group(Single) & Single(Group) \\
\cline { 3 - 5 } & & 2 & Group(No hit) & No hit(Group) \\
\cline { 3 - 5 } & & 1 & Single & Single \\
\hline 2 & \multirow{2}{*}{ No } & 4 & Group & Group \\
\cline { 3 - 6 } & & 3 & Group(Single) & Single(Group) \\
\hline 3 & Yes & 0 & No hit & No hit \\
\hline 4 & No & 2 & Group(No hit) & No hit(Group) \\
\hline \hline
\end{tabular}

The perpendicular distance, $R_{i j}$, between two parallel muons is an observable of interest for analysis of muon bundle events. For $N$ muons of a particular bundle there are $N(N-1) / 2$ perpendicular distances. For tracks through the central detector, the minimum $R_{i j}$ is required to be $8 \mathrm{~cm}$ (see Fig. 3.15). That is, any two tracks within $8 \mathrm{~cm}$ are to be considered here as only one track. The minimum $R_{i j}$ is taken to be $30 \mathrm{~cm}$ for veto shield tracks, reflecting the coarse veto shield spatial resolution. The distributions for inter-muon separations are given in Fig. 3.15 and Fig. 3.16 for the central detector and the central detector plus the shield respectively.

Observed muon bundle multiplicity distributions for the central detector and the shield are shown in Fig. 3.17. Figs. 3.17 (a), (b) and (c) are for the 1990 data sample where (a) shows for the central detector candidate muon tracks only (CLASS 1), (b) shows the CLASS 1 tracks together with candidate muon tracks 


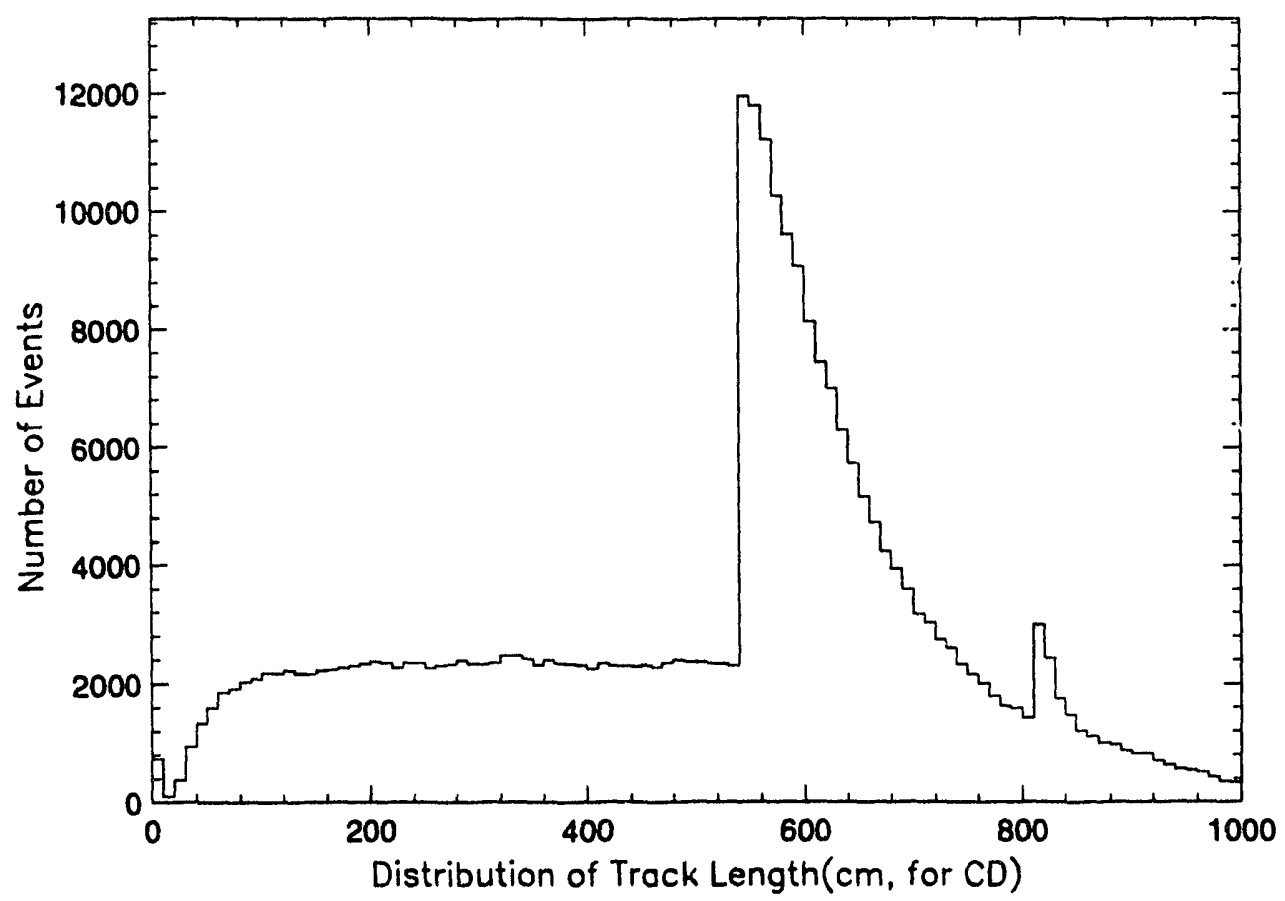

Figure 3.13: Track lengths within the central detector for CLASS 1 muons.

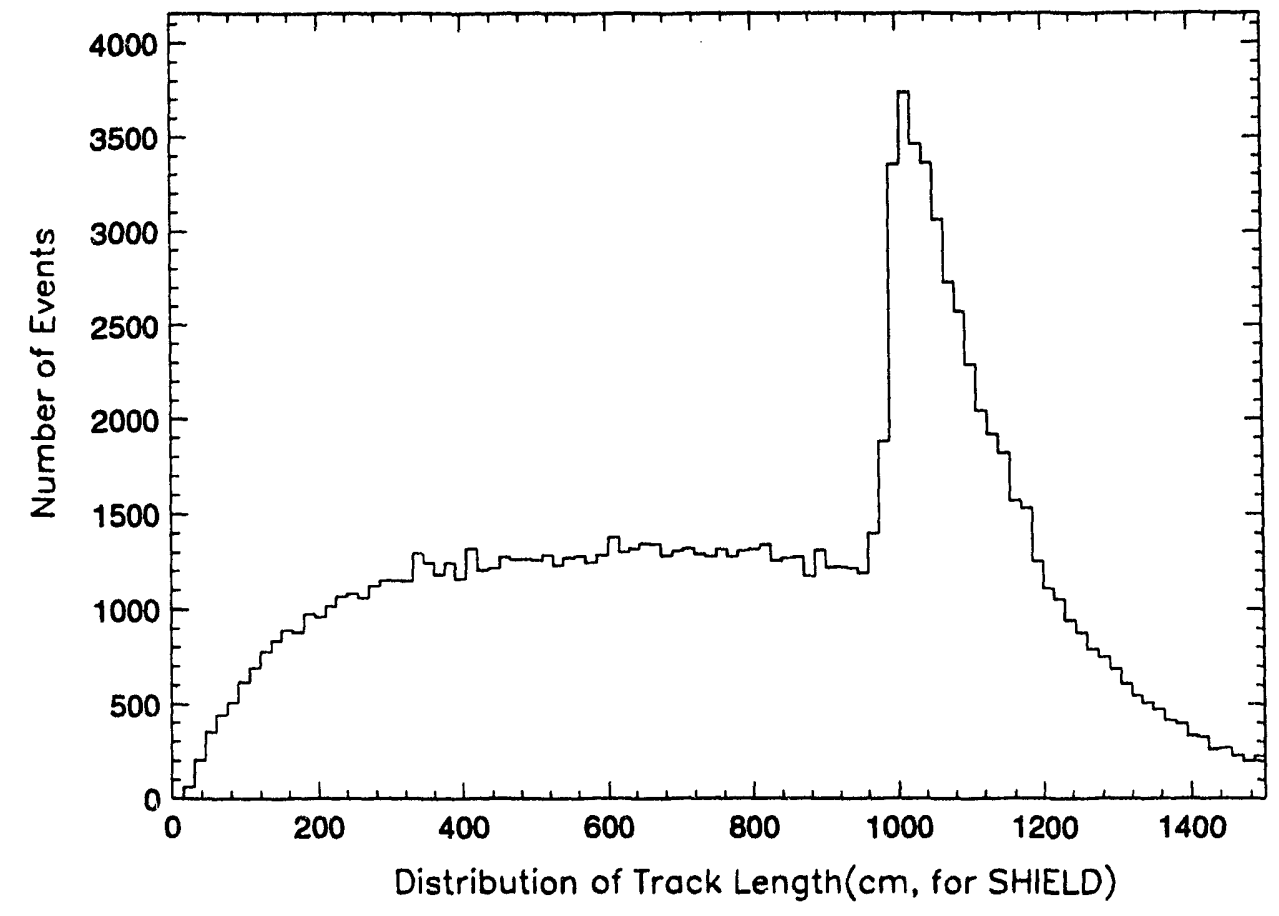

Figure 3.14: Track lengths, enclosed by the shield. 


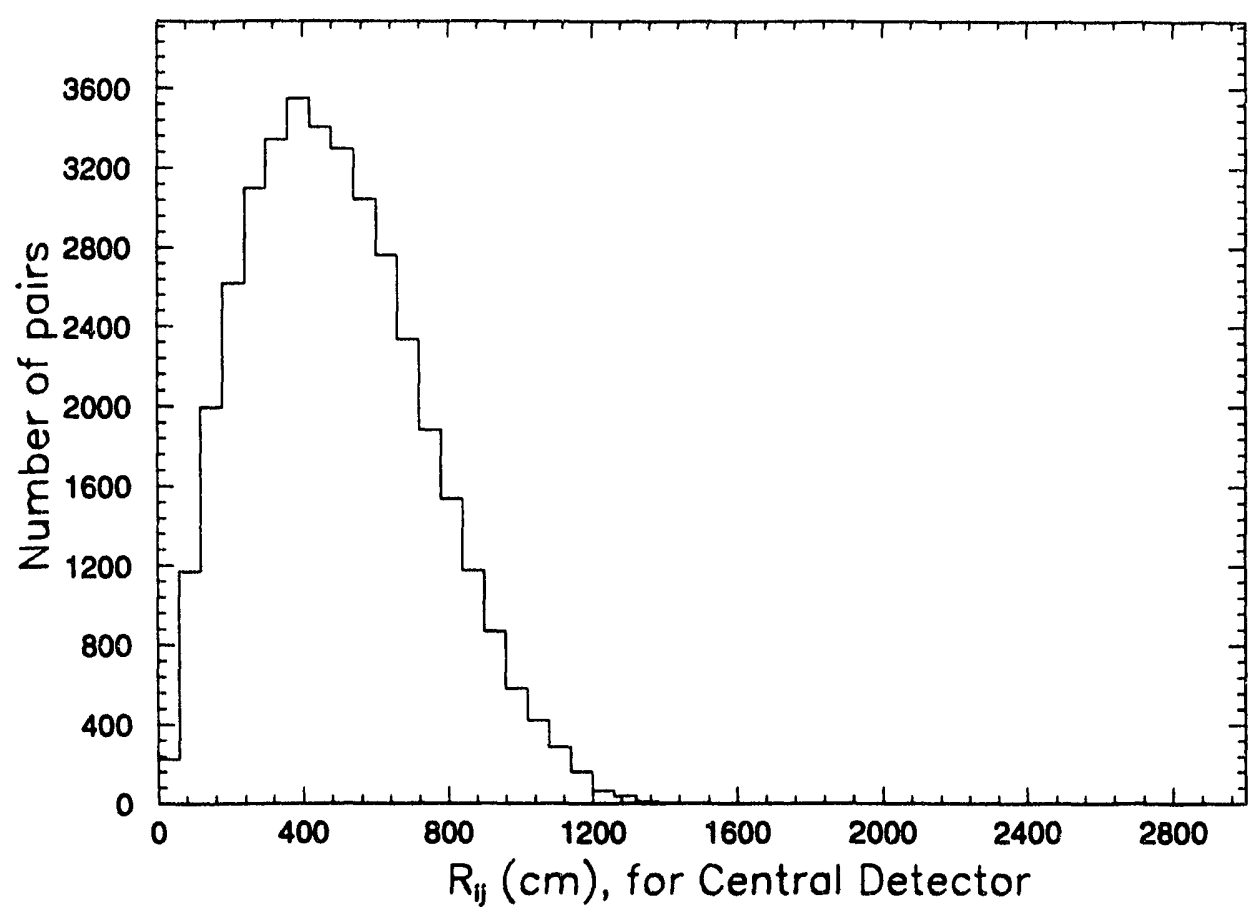

Figure 3.15: Intermuon separation, calculated using Central Detector tracks.

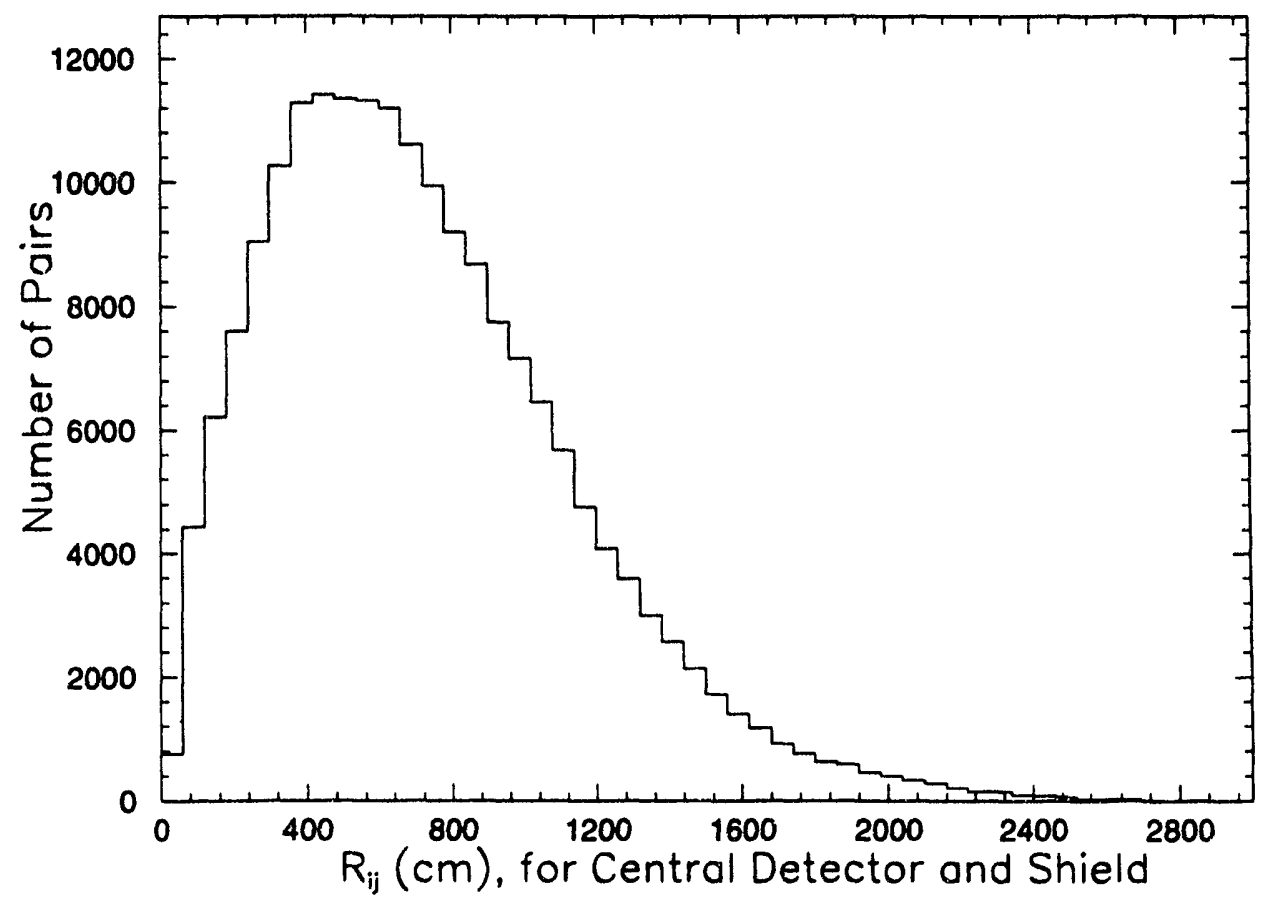

Figure 3.16: Intermuon separation, calculated using Central Detector and Veto Shield tracks. 

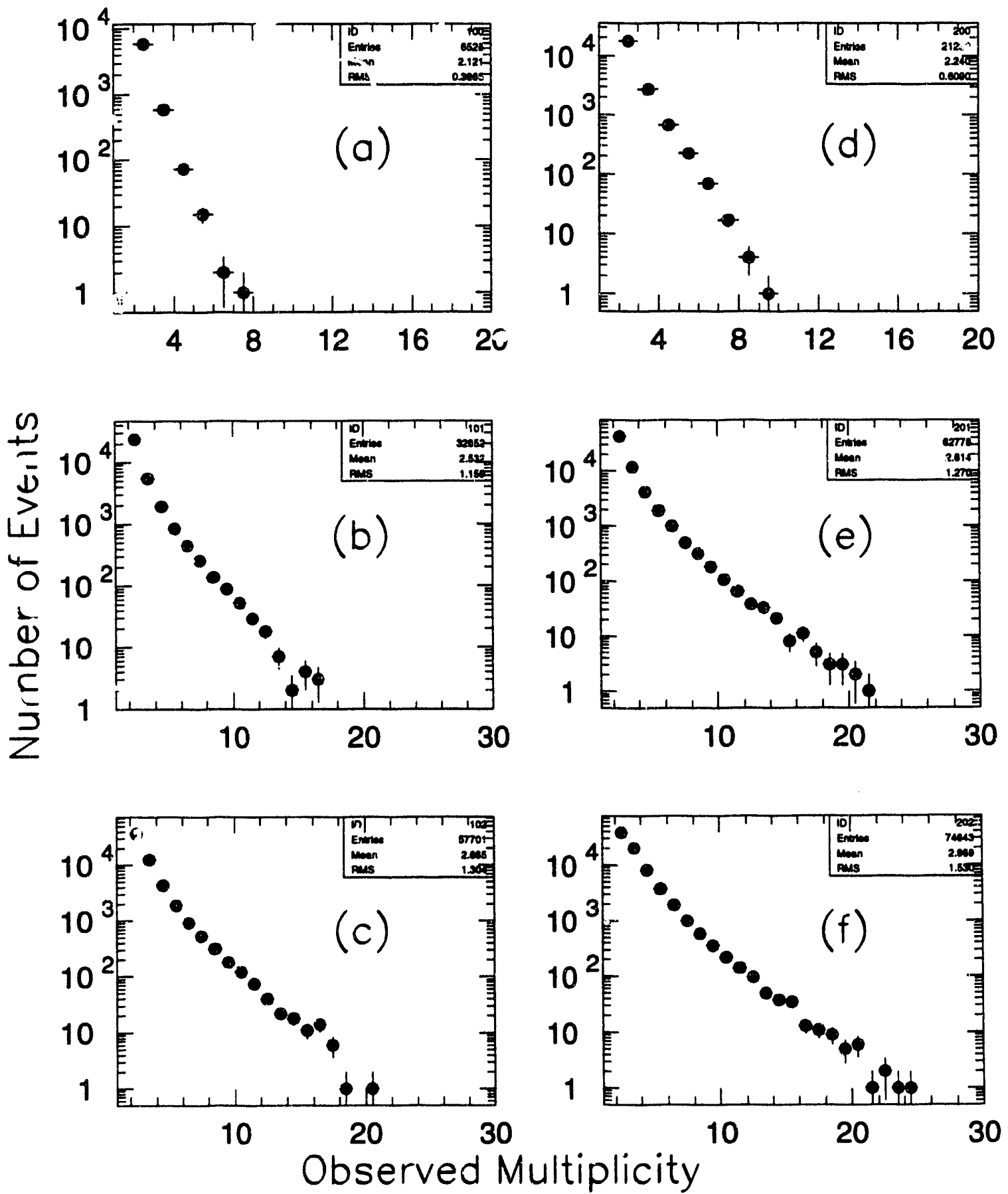

Figure 3.17: Observed multiplicity (a), (d) from the central detector; (b), (e) from the central detector(CLASS 1) and shield (CLASS 2); (c), (f) from central detector, shield and in-time isolated splats (CLASS 4). Figs. (a), (b) and (c) are for the 1990 tata, and Figs. (d), (e) and (f) are for the 1991 data. 
reconstructed using shield hits (CLASS 2) and (c) shows for the CLASS 1, CLASS 2, and together with in-time isc sted splats (CLASS 4). Figs. 3.17 (d), (e) and (f) show similar combinations of candidate muon tracks for the 1991 data sample. The size of the detector and the trigger requirement were changed for the 1991 data sample. Details concerning the trigger conditions will be discussed in Chapter 4 .

Information essential for further analysis is stored in a Data Summary Tape (DST) binary format. Our DST record is a one-dimensional vector array, in which the data for each event are organized into "blocks" which store the information indicated below:

1. Run information such as rur number, event number, date and time for each event.

2. Selected output from the SEARCH processor, including the direction of each muon track in the central detector and the coordinates of exiting points in the central detector as well as in the shield.

3. Information concerning adjacent hit groups such as the number of groups, the time slot and spatial coordinates for each group.

4. Information summarizing the single hits.

5. Information about track reconstruction using all CLASSES of candidate muons.

The DST format is fully described in Appendix B.

\subsection{Displays of Multiple Muon Events}

An example of a muon bundle event is shown in Fig. 3.18. The picture was produced by program MUPLOT, written for use with the commercial graphics software "DISSPLA" to image veto shield hits and reconstructed muon tracks in Soudan 2. Data in the condensed Data-Summary-Tape format were used as input. MUPLOT 
was based upon the software used for data selection, for muon reconstruction, and for creating the DST, and it turned out to be a powerful tool for debugging these codes. Candidate multiple muon events were displayed at Tufts using MUPLOT on a TEKTRONICS workstation augmented with a color CRT terminal. The various projected views and the shield hits in various panels were displayed in different colors in order to clarify the hit patterns of these complex events. Unfortunately, the DISSPLA graphics package is no longer available at Tufts.

In Fig. 3.18, the outer solid-line represents the veto shield, and the smaller dotted outline represents the central detector. Track 1 is detected within the central detector, with exit points shown by open circles. The full circles denote the veto shield exit points of the track extrapolated from the central detector. All the other tracks are reconstructed using veto shield hits only. The adjacent-hit groups are shown by rectangles (solid line in the ceiling, dotted line elsewhere), and single hits are depicted by the straight-line segments. Track 2 is an example of a $M_{\perp}$ combination of shield hits involving the west ceiling and the west floor. The $M_{\perp}$ combinations are reconstructed with small uncertainty in the exit points. Tracks 3 (east ceiling - east floor) and 4 (west ceiling - south wall) are examples of $M_{\|}$ combinations; they have large uncertainty in the exit points in the direction along the veto shield manifolds (east - west direction in this example).

The layout of a full event display is illustrated in Fig. 3.19. The 3-D view is placed into the center surrounded by "unfolded" veto shield super panels; the ceiling on the top, west and east walls are on the right, floor is on the bottom, and the south wall is on the left. The upper and lower right-hand corners show projections of the entire veto shield plus central detector complex, depicting all veto shield hits and reconstructed tracks, as viewed from the north (on top) and from the east (on the bottom).

A display of a two-muon event is shown in Fig. 3.20, and a twelve muon bundle is displayed in Fig. 3.21. In Fig. 3.20, the track which is reconstructed in the central 
detector extrapolates to a point in the east ceiling which is a point of intersection of two perpendicular veto shield modules. The southeast corner of the shield ceiling is the only area of the veto shield with criss-crossed shield manifolds. In this area one can pinpoint the position of a muon track with ease and accuracy. This shield manifold configuration is left over from an early study of single muons passing through the Soudan 2 cavern (Ret. [24]). It is indicative of the muon tracking capability which might eventually be achieved with future upgrades of the Soudan 2 veto shield, as will be mentioned in Chapter 8.

A STING image of a muon bundle with fourteen muons passing through the central detector is shown in Fig. 3.22. STING (Soudan Two INteractive Graphics) is a software package which allows events to be displayed interactively. It provides both an anode pulse plot and a cathode pulse plot, displaying the microstructure of ionization in each drift tube within each central detector calorimeter module. STING also displays positions and timing for shield CTL hits. It has many more features such as indirect energy tagging, using colors to show the amount of ionization deposited in each drift tube, etc. The "space plot" shown in the Fig. 3.22, has three views. The "FRONT VIEW" shows the $\mathrm{Y}-\mathrm{X}$ projection of the event. This is the view that one has when looking at the detector from the north facing south. The "SIDE VIEW" is the Y-Z projection of the same event; this is the view standing on the east, facing west. The "TOP VIEW" is the $\mathrm{X}-\mathrm{Z}$ projection. As discussed earlier, the slope and intercept of a track in the $\mathrm{X}-\mathrm{Z}$ projection and $\mathrm{Y}-\mathrm{Z}$ projection have been used to reconstruct muon tracks in three dimensions. 


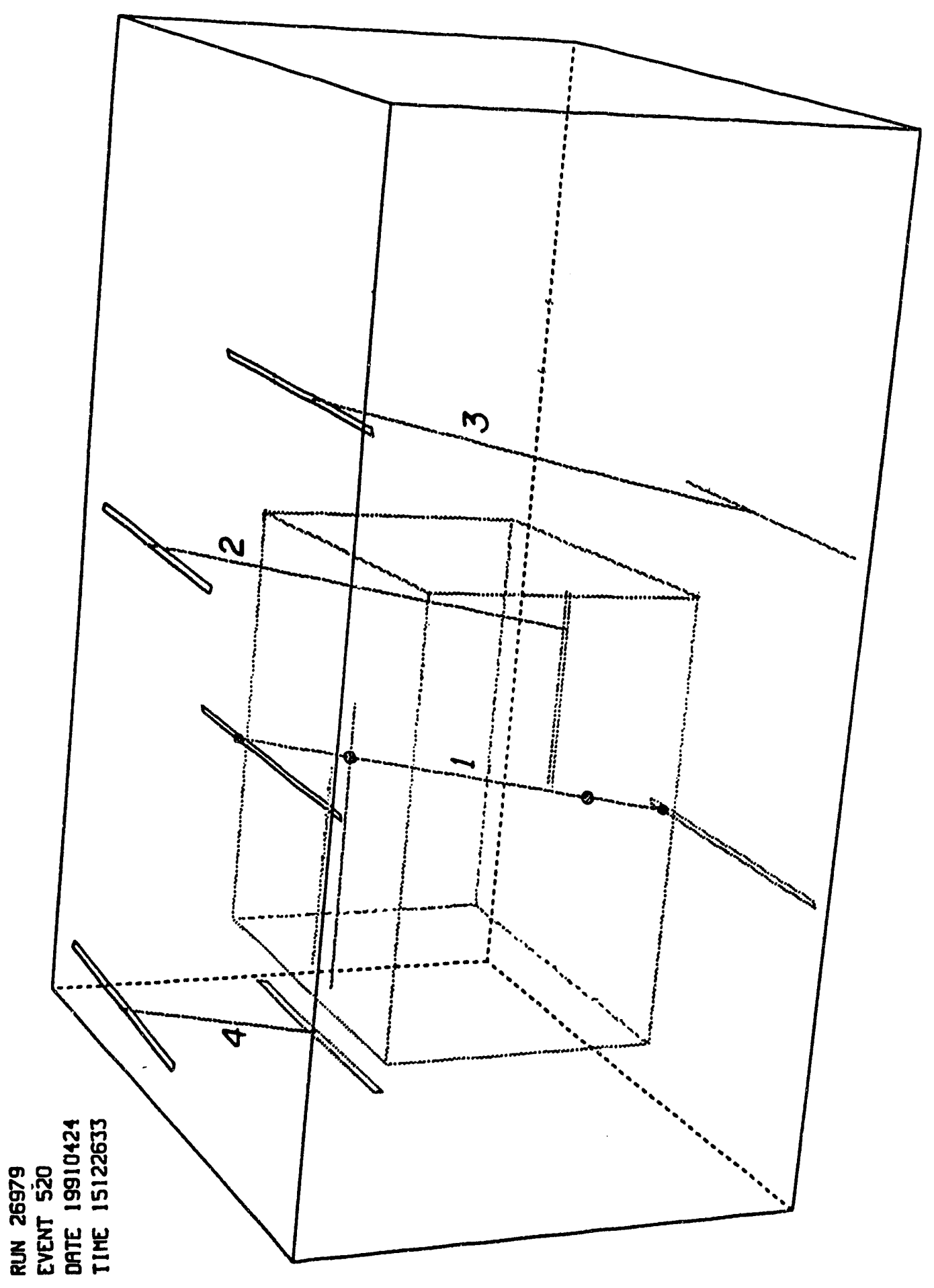

Figure 3.18: Three dimensional view of an event. The numbered tracks are examples of the various classifications of candidate muon tracks. 


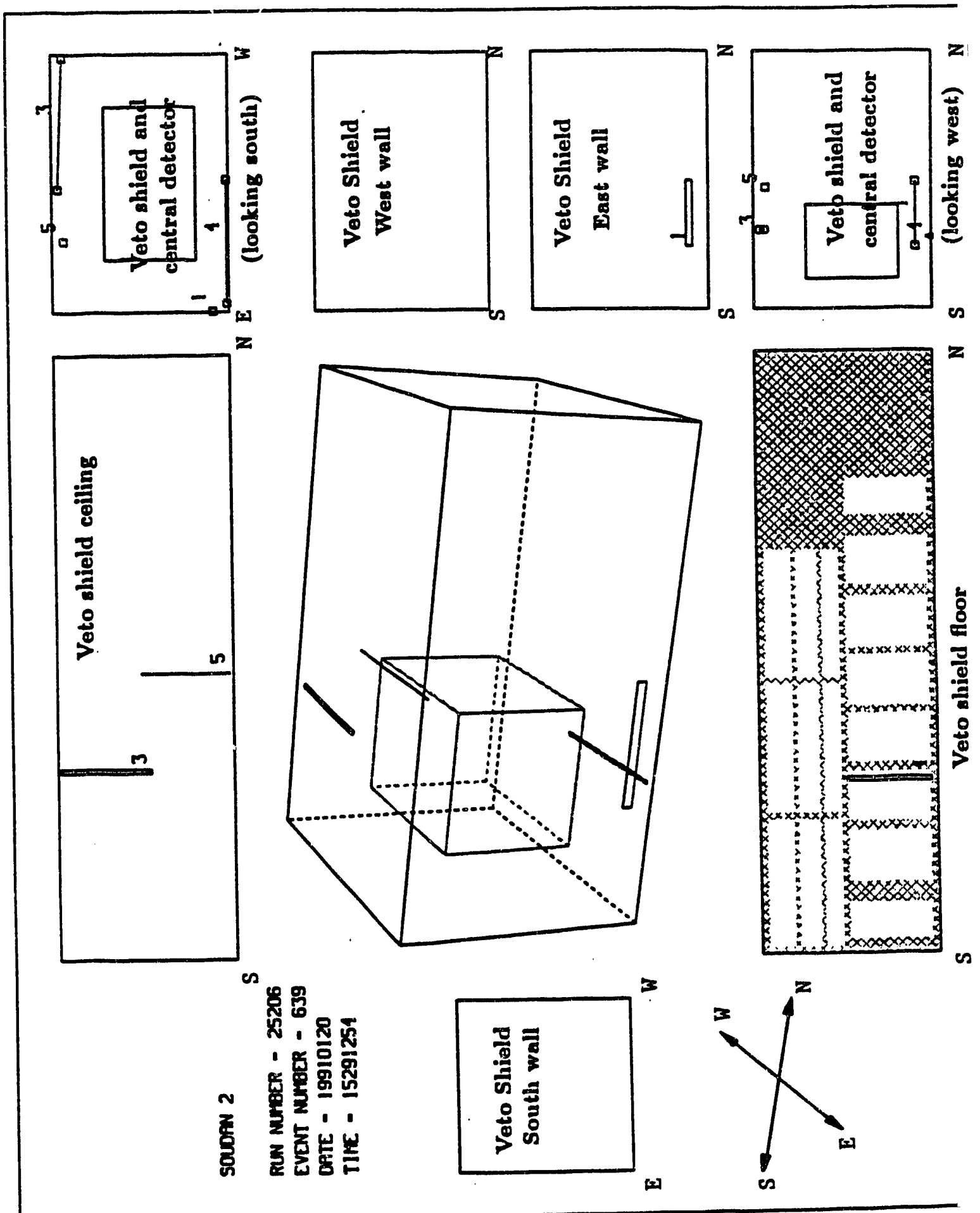

Figure 3.19: An Event Display showing the 3-D view together with the layout (unfolded) of veto shield superpanels. 

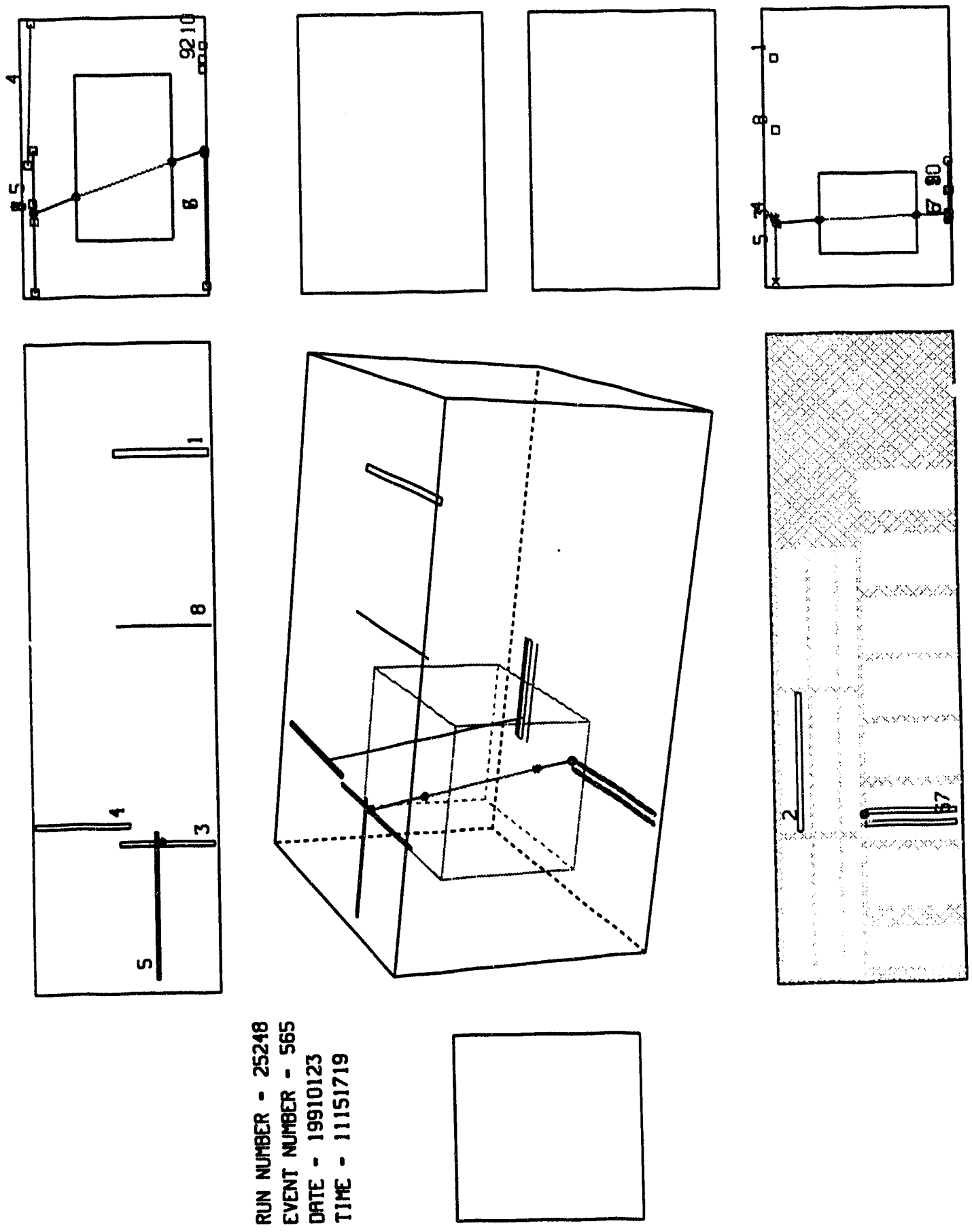

Figure 3.20: An Event Display showing the coincident hit patterns recorded on some veto shield surfaces with two muon tracks. 


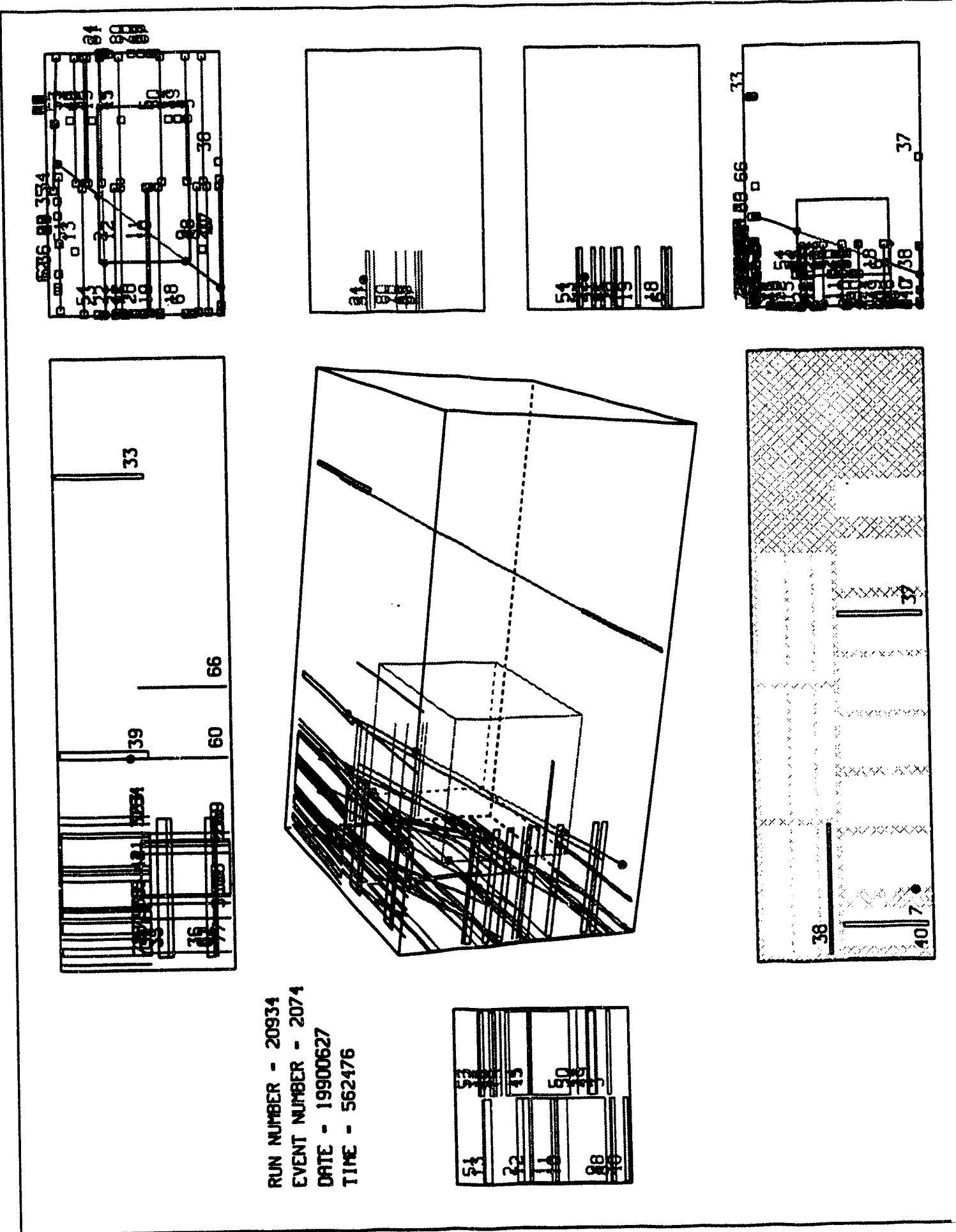

Figure 3.21: An Event Display showing the coincident hit patterns recorded on all veto shield surfaces in a twelve multiple muon event. 


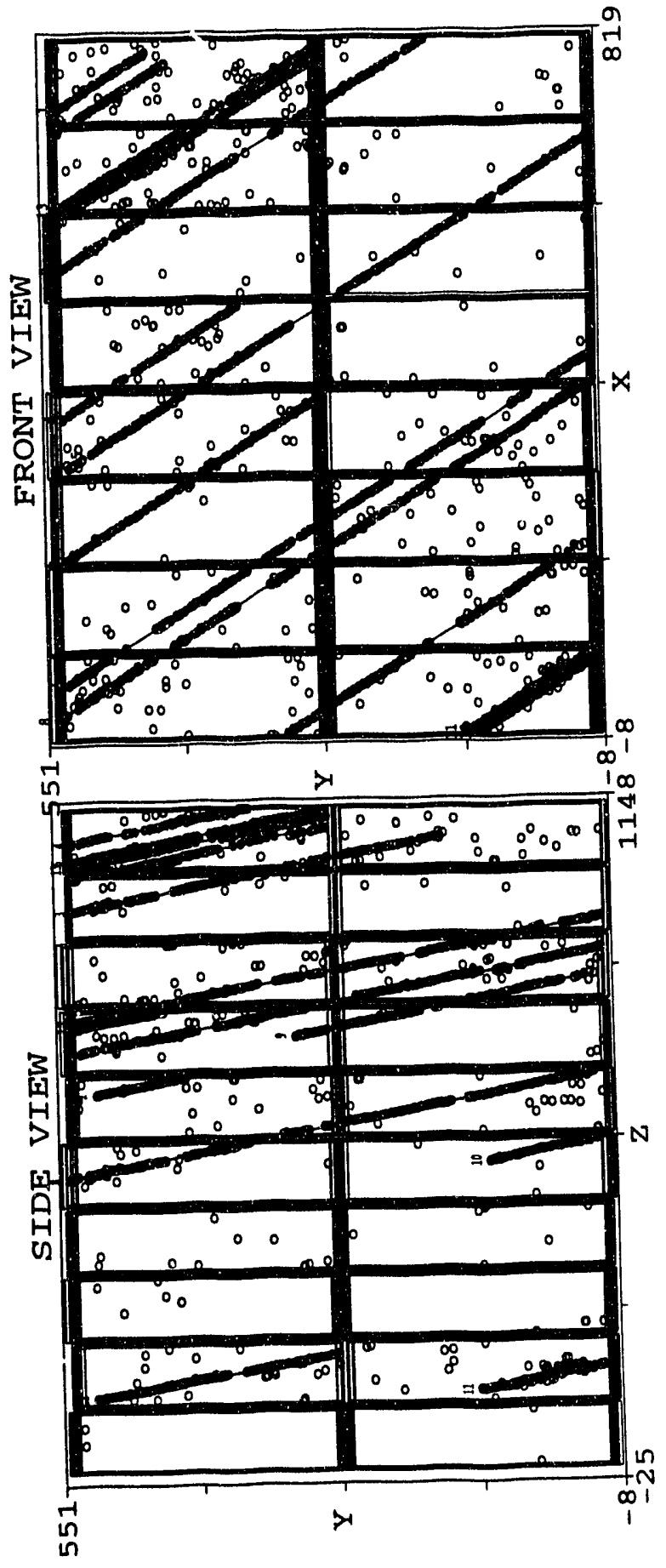

Figure 3.22: STING image of an event which has fourteen muons passing through the central detector. 



\section{Chapter 4 \\ Detector Acceptance, Efficiencies and Uncertainties}

\subsection{Acceptance of the Central Detector}

For the 1990 data sample, the edge trigger requirement, as discussed in Chapter 2 , was set at 7 edges for the anode channels of the central detector. This requirement yields an overall acceptance of $84 \%$ for through-going muons, with the bulk of the losses being due to trigger "holes" along the north-south and east-west directions. The edge trigger condition was embellished for the 1991 data sample by accepting the logical .OR. of 7 anode edges and 8 cathode edges (see section 2.6). As a result, the trigger acceptance increased to $94 \%$ [30]. The improvement can clearly be seen in the azimuthal angle distributions shown in section 4.3 below.

The efficiency for reconstruction of single muons which trigger the central detector, using the processor SEARCH is about $99 \%$ [34]. This efficiency is reasonable for multiple muon event reconstruction with up to four or five muons in the bundle. For higher multiplicity events $(>5)$, the SEARCH reconstruction efficiency for individual muons is somewhat smaller.

\subsection{Efficiency of the Active Shield}

The overall efficiency of the veto shield has been determined using muon tracks which are reconstructed in the central detector and then extrapolated in both directions to the surrounding shield surfaces, where the activity in the shield is examined. 
Table 4.1: Veto shield area as of June 1991.

\begin{tabular}{|l|c|c|c|c|}
\hline \hline Veto shield & $\begin{array}{c}\text { Total area } \\
\left(\mathrm{m}^{2}\right)\end{array}$ & $\begin{array}{c}\text { Area covered } \\
\text { by panels }\left(\mathrm{m}^{2}\right)\end{array}$ & $\begin{array}{c}\text { Area of } \\
\text { the gaps }\left(\mathrm{m}^{2}\right)\end{array}$ & $\begin{array}{c}\text { Percentage } \\
\text { covered (\%) }\end{array}$ \\
\hline East and West Ceiling & 420.0 & 420.0 & 0.0 & 100.0 \\
\hline East Wall & 294.3 & 284.6 & 9.7 & 96.7 \\
\hline West Wall & 294.5 & 284.6 & 9.9 & 96.6 \\
\hline South Wall & 128.1 & 125.8 & 2.3 & 98.1 \\
\hline East and West Floor & 420.0 & 227.8 & 192.2 & 54.2 \\
\hline Total area & 1556.9 & 1342.8 & 214.1 & 86.2 \\
\hline
\end{tabular}

The two factors which contribute to overall shield inefficiency are geometrical losses and electronic inefficiency. Geometry losses arise from the fact that all vertical walls and the floor have gaps between panels; some of these gaps are unavoidable due to requirements of structural supports for the central detector. Many of these gaps were later filled or covered by small "gap filler" units which however were not in place by summer 1991 .

The areas for different shield panels are given in Table 4.1. The fractional area covered by the active shield, out of the total shield outline, is $86 \%$ for the complete veto shield (excluding the north wall), and $98 \%$ for the south wall, $97 \%$ for the east wall and the west wall, and $54 \%$ for the floor. The ceiling is fully covered. The above numbers show that the veto shield has a geometrical acceptance factor which limits muon detection in the shield. Note that the floor superpanel has gaps which are distinctly bigger than those found in the other panels.

Veto shield efficiencies have been calculated based upon scanning of three-dimensional images of the reconstructed tracks. Scanning was carried out for four data runs; only bundle multiplicities of up to 3 muons in the central detector were used so as to minimize pattern recognition problems. A breakdown of the various hit statistics is given in Table 4.2. For a central detector track, the existence of at least one shield hit 
Table 4.2: Number of veto shield hits associated with each central detector muon track, from 376 muon events scanned by physicists.

\begin{tabular}{|l|r|}
\hline Shield Hit Configuration & Number of Tracks \\
\hline 4 layer hits & 499 \\
3 layer(Groups/Single) & 47 \\
2 layer(Groups/No-hits) & 26 \\
2 layer(Groups/Gap) & 47 \\
1 layer(Singles/No-hits) & 1 \\
\hline Total number of tracks & 620 \\
\hline \hline
\end{tabular}

(single hit or adjacent group) was required. However, for any track reconstructed using shield hits only, the track was required to have shield hits at both ends. The veto shield hits can be either group-group or group-single hit combinations. The results are as follows:

For 620 tracks, the number of "hits" expected for a perfect shield is 2,480 . The total number of hits observed is 2,284 , consequently the overall efficiency of the shield, which includes both geometry and electronics, is $92 \%$. For the ceiling panels the overall efficiency (geometry and electronics) is $98 \%$, for the floor panels it is $86 \%$, for the east wall $97 \%$, for the west wall $98 \%$, and for the south wall the efficiency is $97 \%$. The electronic efficiency is $96 \%$. The overall efficiency of the floor panels is higher than the geometrical coverage (54\%) of the floor according to this calculation for two reasons. Because of asymmetry of the central detector with respect to the shield layout, muon tracks are preferentially located within the central detector area. Underneath the central detector, however, most of the floor is covered. The uncovered north floor area adversely affects the floor's geometry efficiency but this portion of the floor is less frequently traversed by muons from triggering bundles.

The electronic efficiency of the shield can also be investigated with high statistics using tallies of hits observed with CLASS 1 tracks in the central detector. For this 
Table 4.3: Run statistics for 54,035 events with two or more candidate muons from the 1991 data, used to calculate efficiency of the shield.

\begin{tabular}{|l|r|}
\hline \hline Shield Hit Configuration & Number Tracks \\
\hline 4 layer hits & 39,991 \\
3 layer hits & 7,645 \\
2 layer (Singles/Sing'es) & 470 \\
2 layer (Groups/No-hits) & 15,834 \\
2 layer (Groups/Gap) & 8,049 \\
1 layer (Singles/No-hits) & 1,240 \\
1 layer (Singles/Gap) & 588 \\
0 layer (No-hits/No-hits) & 1,158 \\
Total number of tracks (CLASS 1) & 73,817 \\
\hline \hline
\end{tabular}

analysis, a total of 54,035 events from May through July 1991 have teen used. The events contained 73,817 central detector tracks classified as CLASS 1 tracks (see Table 4.3), i.e. accompanied by veto shield hits, and 1,158 central detector tracks classified as CLASS 3, i.e. without any veto shield hits. Only tracks with zenith angle between $15^{\circ}$ and $60^{\circ}$, and with recorded track length greater than $100 \mathrm{~cm}$, are included.

With full shield coverage, every central detector track should be accompanied by signals in four channels of veto shield electronics, two coming from neighboring layers of veto shield modules within an adjacent group at each end of the (extrapolated) track. All possible configurations of detected and missing veto shield hits are categorized in Table 3.2, and the corresponding track counts are brought together in Table 4.3. Note that we have separated the counts of tracks which have no shield signal on one side into two categories, one for the "true" No-hits case, where the central detector track is extrapolated to an existing veto shield module which did not fire, and a second category for tracks, which pointed into a gap between veto 
shield panels.

Fcr 73,817 tracks in a hermetic shield with $100 \%$ electronic efficiency, the number of veto shield hits arising from four-layer coincidences should be 295,268 . The total number of hits observed is 233,433 . This would indicate that the overall shield efficiency is $79 \%$ (78\%) if we exclude (include) CLASS-3 tracks, a value that is lower than the $92 \%$ obtained for the smaller scanned sample. In particular, within the scanned sample, only $4 \%$ of tracks are of the Hits/No-hits categories (Groups/Nohits and Singles/No-hits), $8 \%$ are Hit/Gap tracks, and no No-hits/No-hits tracks were observed. In comparison, in the 1990 (1991) sample, the fraction of Hits/Nohits tracks is $13 \%(24 \%)$, Hits/Gap tracks form $13 \%$ (12\%) of the total, and there are $0.7 \%(2 \%)$ No-hits/No-hits tracks. The quality of data seems to be lower in the overall sample, :nd worse in 1991 than in 1990.

The reason for this discrepancy lies in the data processing. The 1990 data were processed with Release-13 SEARCH, which was conservative, while Release-15 SEARCH was used for the 1991 data. In this verson, many more spatially limited collections of hits were reconstructed as tracks. In our analysis, the length of the track within the central detector (as included in the DST) was calculated as a distance between the inferred exit points of the track from the outer surface of the detector, which in some cases does not represent the distance between the actual end hits within the detector. Consequently, the tracks which are in the CLASS1 category include the following track types: (i) real through-going muons with associated shield hits at both ends; (i:; real through-going muons with associated shield hits at one side only, where the shield failed to respond on the other side due to electronic inefficiency or due to a presence of a gap; (iii) stopping muons with associated shield hits on one side; and (iv) extrapolated shorter tracks which may have associated shield hits that are accidental, caused by natural radioactivity in the surrounding rock, or by $\delta$-rays from nearby muon.

Separating real through-going muons from the tracks in categories (ii), (iii), 
and (iv) is impossible at this point because the actual lengths within the central detector are not available on the DST. As a consequence, candidate muon tracks of these categories, as well as the No-hit/No-hit tracks, have been eliminated from the further analysis for multiple muon studies. As a result, we have included for further analysis as good through-going muon tracks $64 \%$ of the total number of candidate muon tracks.

We next calculate the veto shield electronic efficiency for the data used in our final analysis using central detector tracks with shield hits at both ends. We obtain a value of $96 \%$ which agrees with the efficiency obtained from the sample of scanned events.

Lastly, we estimate that the tracks eliminated from the analysis contain $9 \%$ of the true through-going muon tracks (based upon the scanned events). By eliminating some good muon tracks from the total number of through-going muons with the cuts described above, a potential source of systematic error is introduced, which will be examined in Section 6.3.

\subsection{Zenith Angle and Azimuthal Angle Distributions}

We are able to detect muons in most directions with good acceptance. However, there are a few specific directions with reduced acceptance. The angular acceptance of the central detector is described here in terms of the zenith and azimuthal angles. The zenith angle is the angle of a muon track (unit vector $\hat{u}$ in Fig. 4.1) with respect to the vertical direction ( $\beta$ in Fig. 4.1), and the azimuthal angle is the angle between the projection of a track onto the XZ-plane, $u_{X Z}$, and the Z-axis ( $\phi$ in Fig. 4.1). We calculate the cosine of the azimuthal angle, $\phi$,

$$
\cos \phi=\frac{\cos \gamma}{\sin \beta}
$$

where $\beta$ is the zenith angle, and $\cos \gamma$ is the directional cosine with respect to the $Z$ axis. Distributions in $\beta$ and $\phi$ are shown in Figures 4.2, 4.3, and 4.4. As seen in Fig. 
4.2, most of the detected muons are obsorved to be incident with zenith angles of $30^{\circ}$ $-40^{\circ}$. We note that no significant difference is observed between zenith distributions in the 1990 and 1991 data. In Fig. 4.3 we see pronounced gaps in the $\phi$ distribution, as well as differences between the 1990 and 1991 data.

Azimuthal angle distributions for all zenith angle are shown for 1990 and 1991 data in Figs. 4.3(a) and (b). Fig. 4.4 shows the azimuthal angle distribution for three different zenith angle range from $0^{\circ}$ to $20^{\circ}$, from $20^{\circ}$ to $40^{\circ}$, and from $40^{\circ}$ to $60^{\circ}$. The distributions show that the detector acceptance varies with the muon direction. In the north - south and east - west directions the acceptance is poor for several reasons:

(1) Central detector geometry: There are cracks between adjacent detector modules which are approximately $10 \mathrm{~cm}$ wide and lie along the north - south and east - west directions. Muons which pass through the detector in such a way that most of their trajectory is passing through a crack will not be detected.

(2) Triggering requirement [35]: As described in Section 2.6, at least 7 (8) anodes (cathodes) within any 16-electrode range within $t_{\max }=72 \mu \mathrm{s}$ must fire in order for the event to be recorded. This requirement eliminates tracks that are nearly parallel to the drift direction or to the wire-plane. If we take the anodes as an example, the minimum angle with respect to the drift (north - south) direction is determined by the condition

$$
\tan \gamma_{\min }=\frac{\left(n_{\text {edges }}-1\right) \times \text { anode spacing }}{t_{\max } \times v_{d r i f t}},
$$

and the maximum angle by the condition

$$
\tan \gamma_{\max }=\frac{(16-1) \times \text { anode spacing }}{\left(n_{\text {edgea }}-1\right) \times 600 \mathrm{~ns} \times v_{\text {drift }}}
$$

representing the requirement that only one edge can occur in any one trigger-clock period of $600 \mathrm{~ns}$. For anode spacing of $1.48 \mathrm{~cm}$ and drift velocity of $0.6 \mathrm{~cm} / \mu \mathrm{s}$ we obtain $\gamma_{\min }=11.6^{\circ}$, and $\gamma_{\max }=84.4^{\circ}$. 

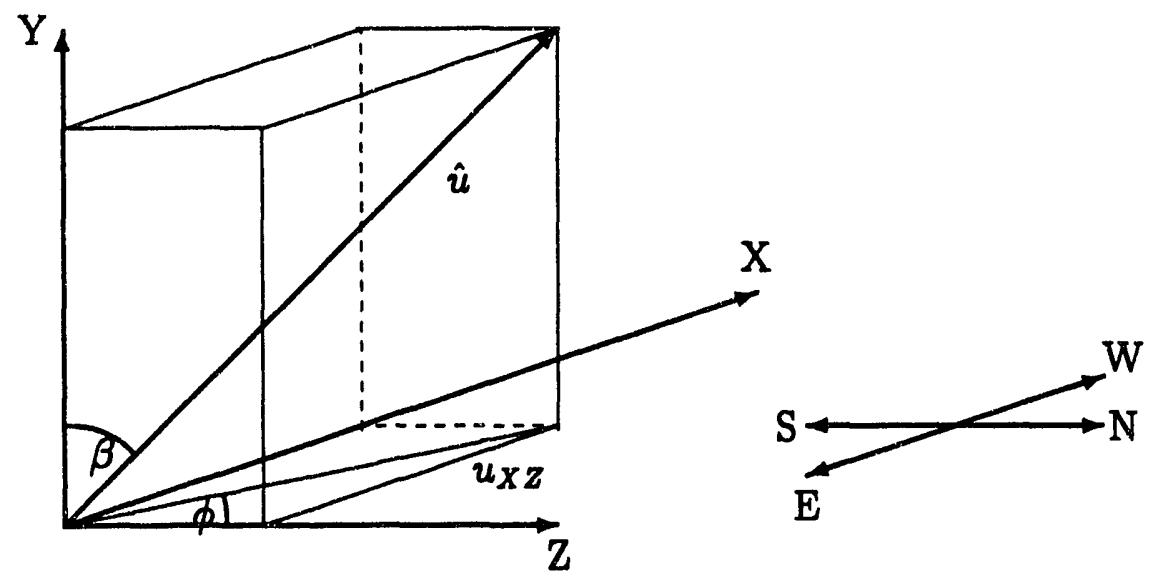

Figure 4.1: Coordinate system for the Soudan-2 cavern: $\beta$ is the zenith angle and $\phi$ is the azimuthal angle.

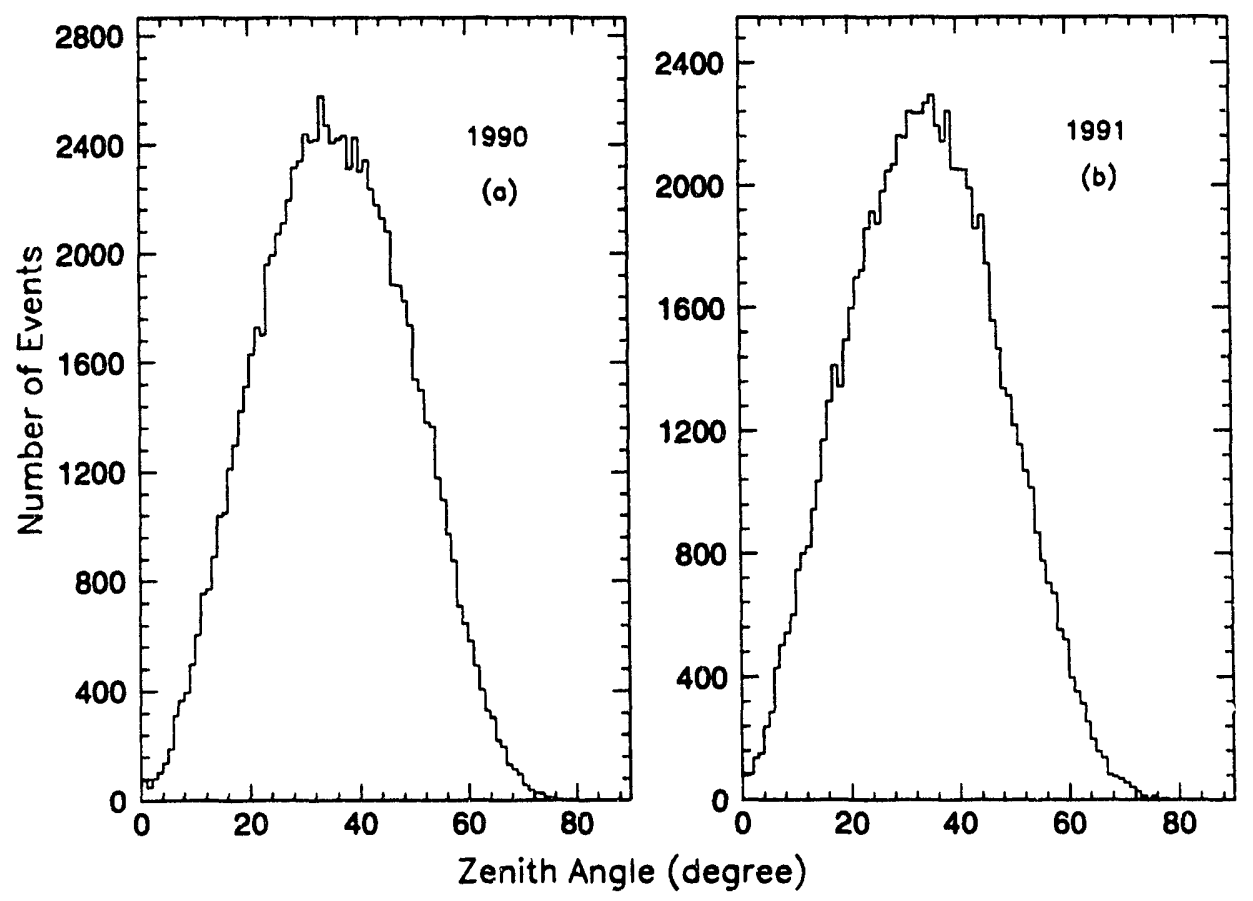

Figure 4.2: Distributions of zenith angle for muon bundles. 


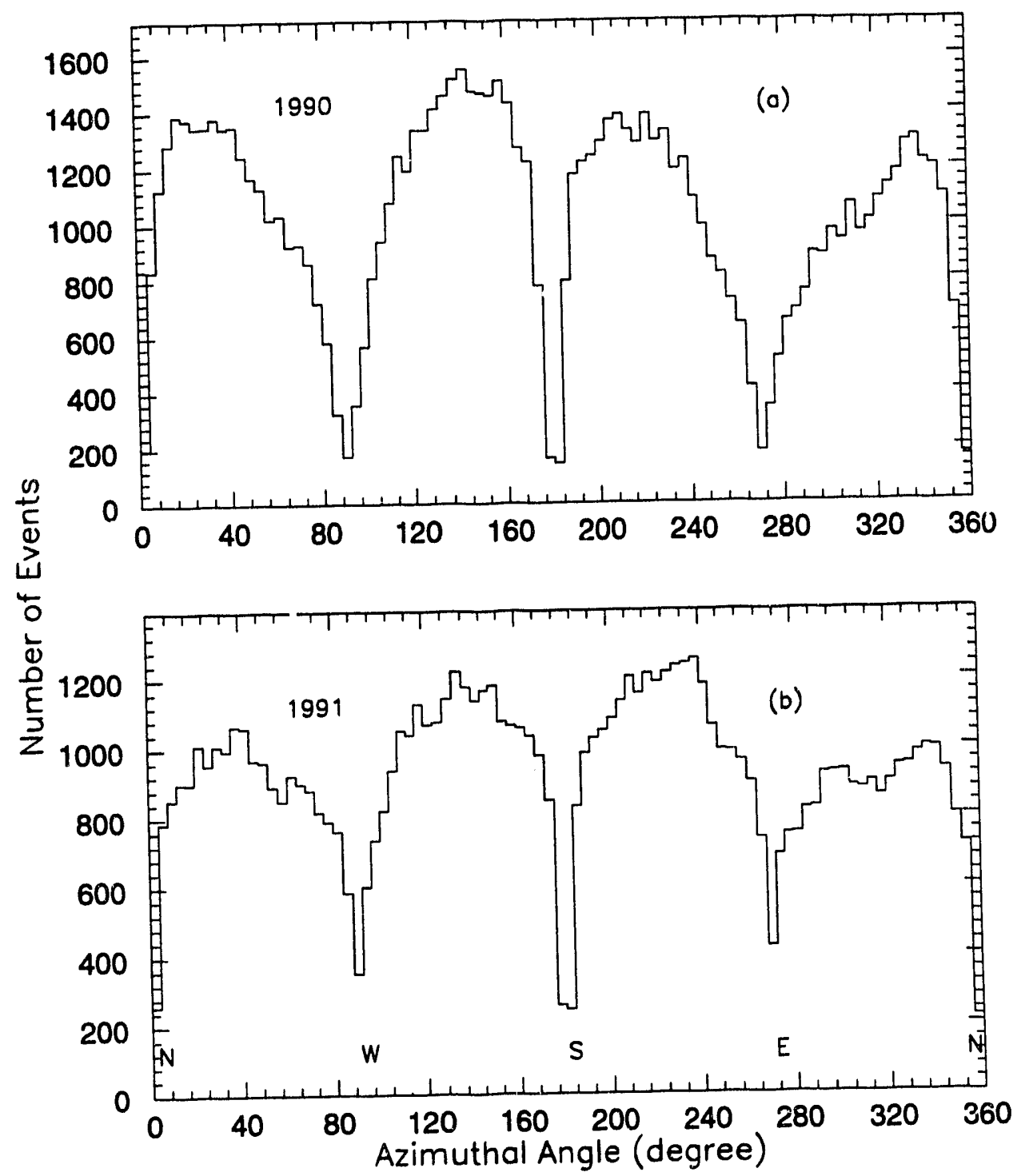

Figure 4.3: Muon azimuthal angle distributions for 1990 and 1991 data samples. The distributions are integrated over all zenith angles. 

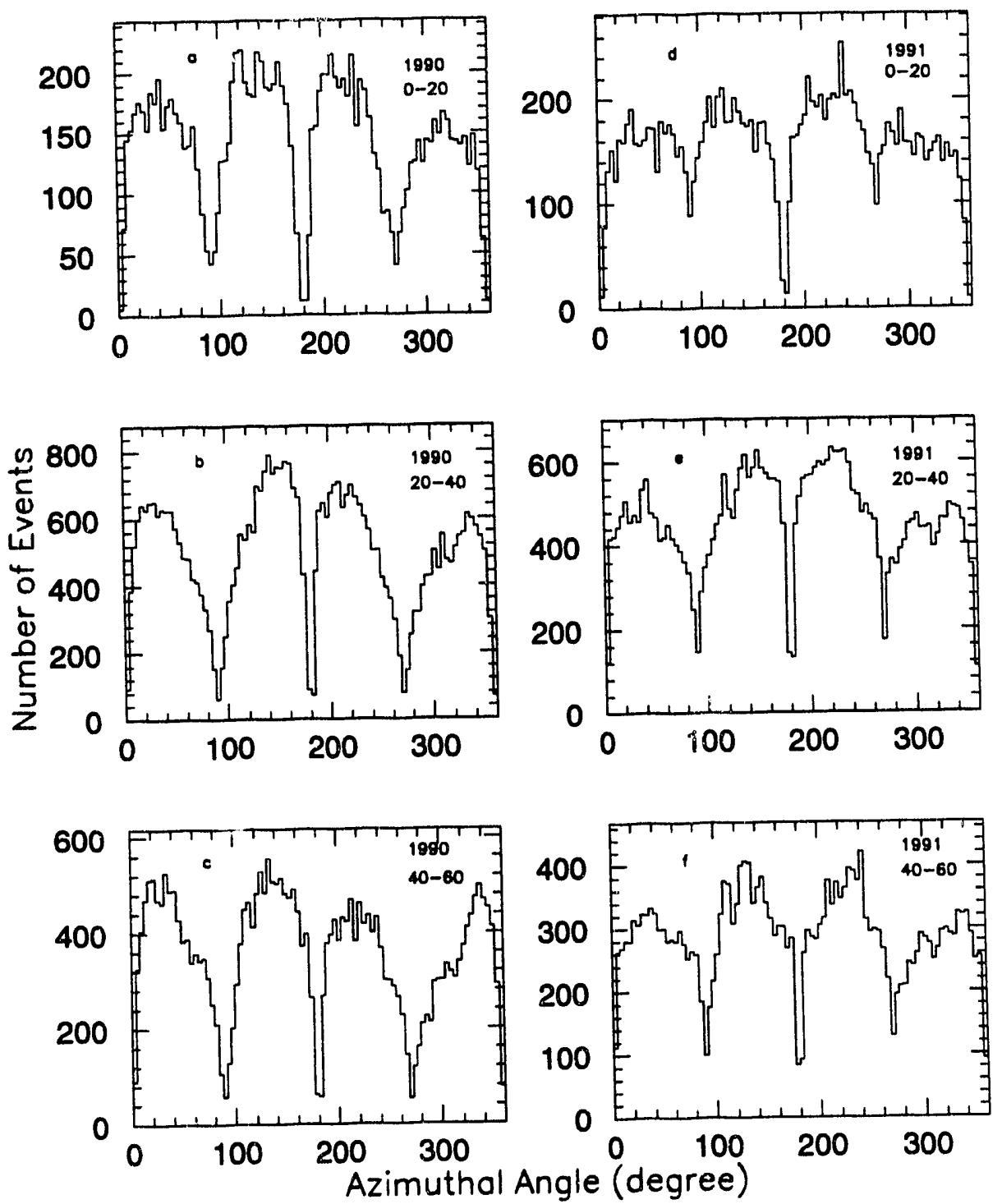

Figure 4.4: Muon azimuthal angle distributions binned according to zenith angle, for 1990 and 1991 data samples. 


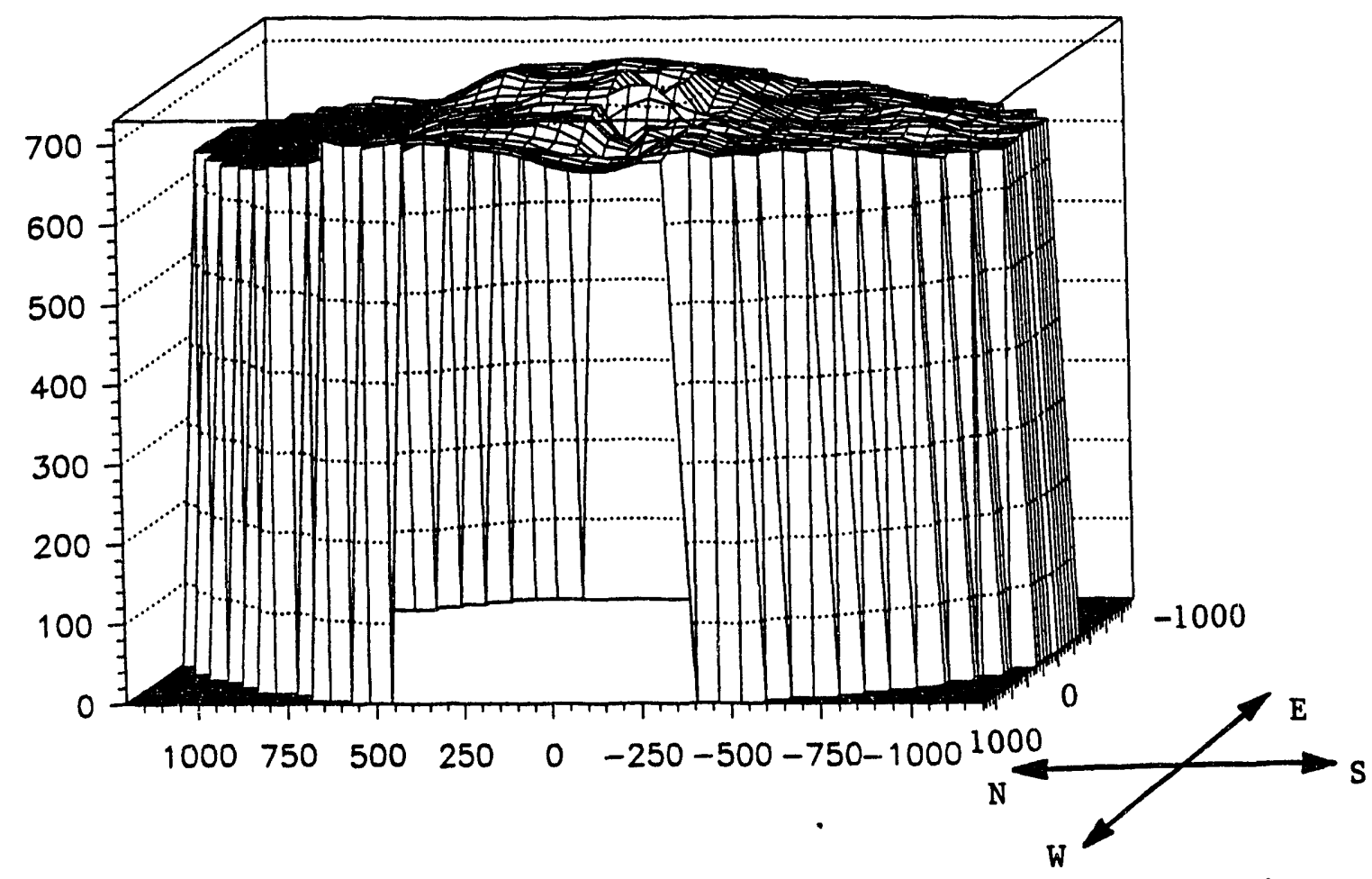

Figure 4.5: Shape of the terrain above the Soudan 2 detector. (All distances ar in meters.)

(3) Track reconstruction: Tracks with very few anode hits or with very few cathode hits are difficult to reconstruct.

Detector acceptance is greatly improved for the 1991 data when cathode pulses were included in the trigger definition along with the anode triggering requirement, as described in Section 4.1. As a result, the acceptance "holes" were narrowed down and partially filled, but not completely eliminated.

The surface above Soudan 2 is not flat; rather, there is a ridge running along the east-west direction (see Fig. 4.5). The effect of variable overburden is readily seen by looking at the differences in the relative event rates which are higher for the $\mathrm{SE}$ and SW quadrants compared to the NE and NW quadrants in Fig. 4.3. Above the south side of the detector, the surface falls off quickly. Above the north side, the land on the surface extends at the same altitude for a longer distance, thus yielding more earth overburden above the detector in the northern direction. 


\subsection{Error Analysis}

Errors in spatial coordinates $x, y, z$ associated with muon tracks are based upon the errors assigned to slopes and intercepts of tracks which have been reconstructed by the SEARCH algorithm. Details concerning the error calculations are given in Appendix C. Recall from Chapter 3 that there are three different types of candidate muon tracks, namely central detector tracks, $M_{\perp}$ shield tracks, and $M_{\|}$shield tracks. The central detector has excellent resolution (1 cm for all spatial coordinates), consequently tracks reconstructed within the central detector have small errors (see section 4.4.1). The veto shield provides relatively poor resolution. Errors for the track coordinates determined by the active shield depend on the track type, $M_{\perp}$ or $M_{\|}$type (see section 4.4.2). Errors on the intermuon separation $R_{i j}$ have been calculated for all combinations of tracks which traverse the central detector and/or the shield. A weighting factor $1 /\left(\Delta R_{i j}\right)^{2}$ is introduced wherever distributions of intermuon separation are plotted.

\subsubsection{Errors on $R_{i j}$ for Central Detector Muons}

The distribution of calculated errors on intermuon separation involving pairs of muon tracks reconstructed using the central detector (CLASS 1 muons) is shown in Fig. 4.6(a). The distribution peaks around $3 \mathrm{~cm}$ and extends up to $30 \mathrm{~cm}$, which is about one and a half veto shield manifold widths. The average intermuon separation for central detector tracks is nearly 4.9 meters, and so the error on $R_{i j}$ is typically less than one percent for pairs of such tracks.

\subsubsection{Errors on $R_{i j}$ for Shield $M_{\perp}$ and $M_{\|}$Muons}

Fig. 4.6(c) shows the distribution of errors assigned to separations $R_{i j}$ among the $M_{\perp}$ type tracks only. The distribution peaks at about $14 \mathrm{~cm}$ and extends to $350 \mathrm{~cm}$, which is half the length of a veto shield manifold. However the distribution 
falls mostly within $30 \mathrm{~cm}$, which is 1.5 shield manifold module widths. The average intermuon separation is 7.1 meters when shield tracks and central detector tracks are combined together. The average percentage error on $R_{i j}$ is $4 \%$ when $M_{\perp}$ type tracks are involved, and $18 \%$ when the track pairs involve $M_{\|}$type tracks.

Fig. 4.6(d) shows the error distribution for combinations when only $M_{\|}$type tracks are used. The error $\Delta R_{i j}$ is seen to be much larger in this case. Although the distribution peaks at $30 \mathrm{~cm}$, it does not fall off at higher $R_{i j}$ values. The contribution of $M_{\|}$type pairs to the $R_{i j}$ distributions for all reconstructed muons is about $20 \%$. Since we weight each $R_{i j}$ by the inverse square of its error, the pairs of $M_{\|}$type tracks do not exert an undue influence on our analysis distributions. 


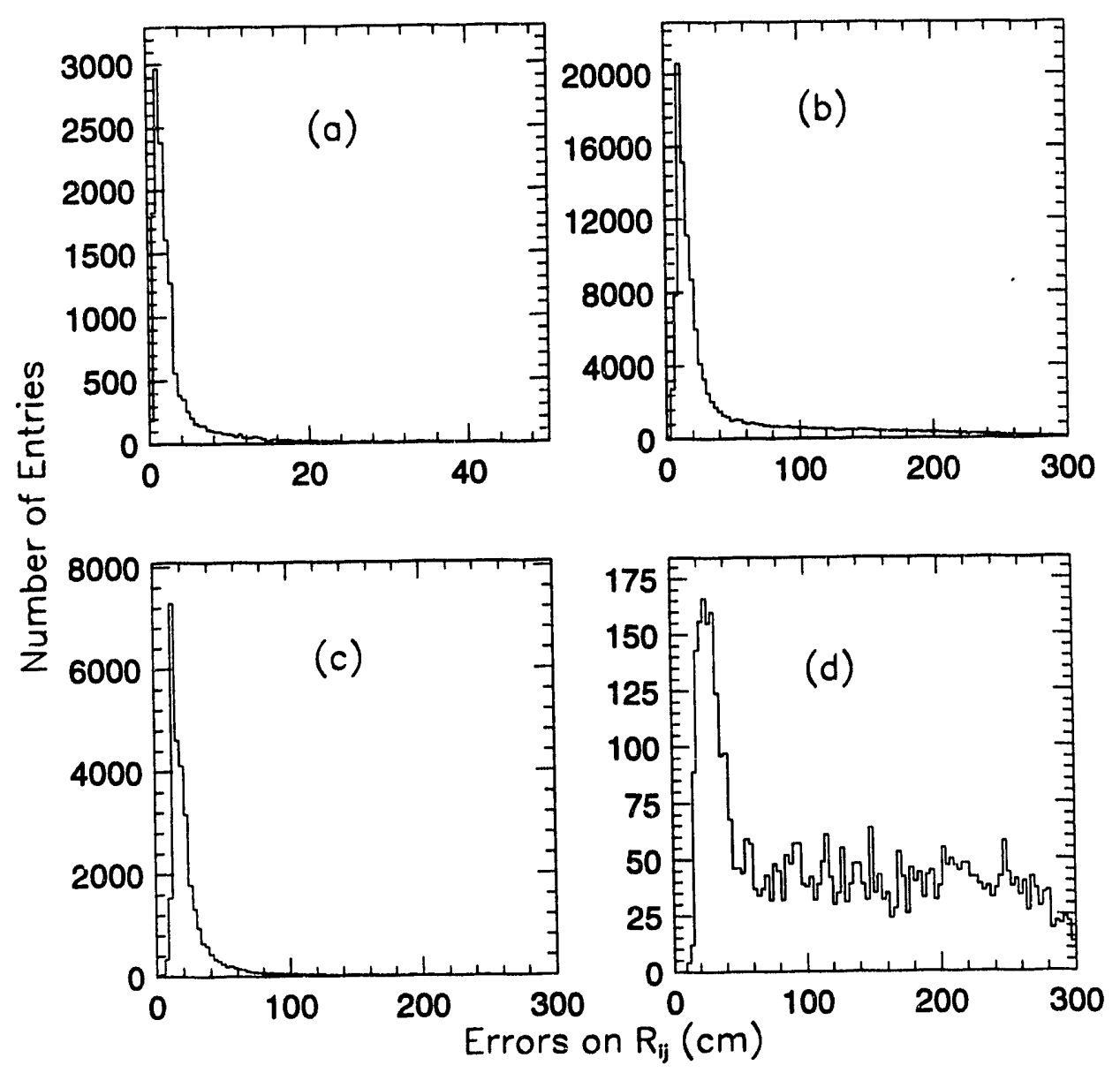

Figure 4.6: Errors on $R_{i j}$ (a) associated with pairs of central detector tracks, (b) with combinations involving an $M_{\perp}$ and an $M_{\|}$candidate muons, (c) with pairs of $M_{\perp}$ candidate muons only, and (d) with pairs of $M_{\|}$candidate muons only. 


\section{Chapter 5 \\ Simulation of Underground Muon Bundles}

\subsection{Overview of the Monte Carlo Simulation}

To relate our observations of muon bundles at a depth of 2090 mwe to the properties of the primary cosmic ray flux, we used Monte Carlo simulations based upon parametrizations given by C. Forti et al. [37]. The parametrizations are based upon detailed, event-by-event simulations of atmospheric cascades. To facilitate comparison, the simulations used for our analysis are simplified, using techniques described below. Our simulation provides rates and distributions of coircident multiple muons to be expected in the Soudan 2 detector system.

An energy of the primary cosmic ray nucleus is selected randomly according to the cosmic ray energy specturum (Section 5.1.1). For purpose of simulation, the primary cosmic ray nuclei are organized into five groups; these include protons $(A=1)$, helium $(A=4)$, the CNO group (average $A=14)$, the $\mathrm{Mg}-\mathrm{Si}$ group $(A=28)$, and iron $(A=56)$. For each primary energy generated, one calculates - for each group of nuclei - a probability that a nucleus is incident at the given energy according to the differential flux of the model being used (see Eq. 5.1). The simulation then selects a particular nucleus in proportion to the probabilities calculated.

The threshold energy $E_{\mu}^{\text {th }}$ at the surface required to give a muon track at the depth of the detector is calculated. The threshold $E_{\mu}^{\text {th }}$ depends on zenith angle $\theta$, the azimuthal angle $\phi$, and the slant depth. The zenith and the azimuthal angles 
are generated isotropically according to

$$
\cos \theta=\cos \theta_{\max }+s \cdot\left(1-\cos \theta_{\max }\right)
$$

and

$$
\phi=s \cdot 2 \pi
$$

where $s(0.0<s<1.0)$ is a random number, and $\theta_{\max }$ is the maximum zenith angle allowed for the simulation, $\theta_{\max }=60^{\circ}$. Isotropic generation of the directions of muons incident upon the Earth's surface above the detector is found to yield an accurate representation of muon directions observed at the detector - see Fig. 6.1. The average number of muons for our depth is calculated using a function described in Section 5.1.3. For each muon within a bundle, the perpendicular distance from the bundle core is selected according to the power law described in Section 5.1.4. Then the bundle core with its associated muons is distributed randomly yielding as "detected muons" those which pass through the central detector and/or the shield. The detected muons from simulated bundles are written onto a DST with format nearly identical to the data DST format.

For purposes of simulation, the central detector and the shield are treated as rectangular parallelepipeds with dimensions $8 \times 11 \times 5 \mathrm{~m}$ and $14 \times 30 \times 10 \mathrm{~m}$ respectively. Coordinates of entering and exiting muon tracks through the shield are transformed into panel number, tube number and in-or-out channels, which is the same form in which real muon hits are recorded. At least one muon is required to pass through and be recorded by the central detector, in order that a muon bundle be considered for further analysis. The muon(s) through the central detector must satisfy the trigger conditions for the actual data. 


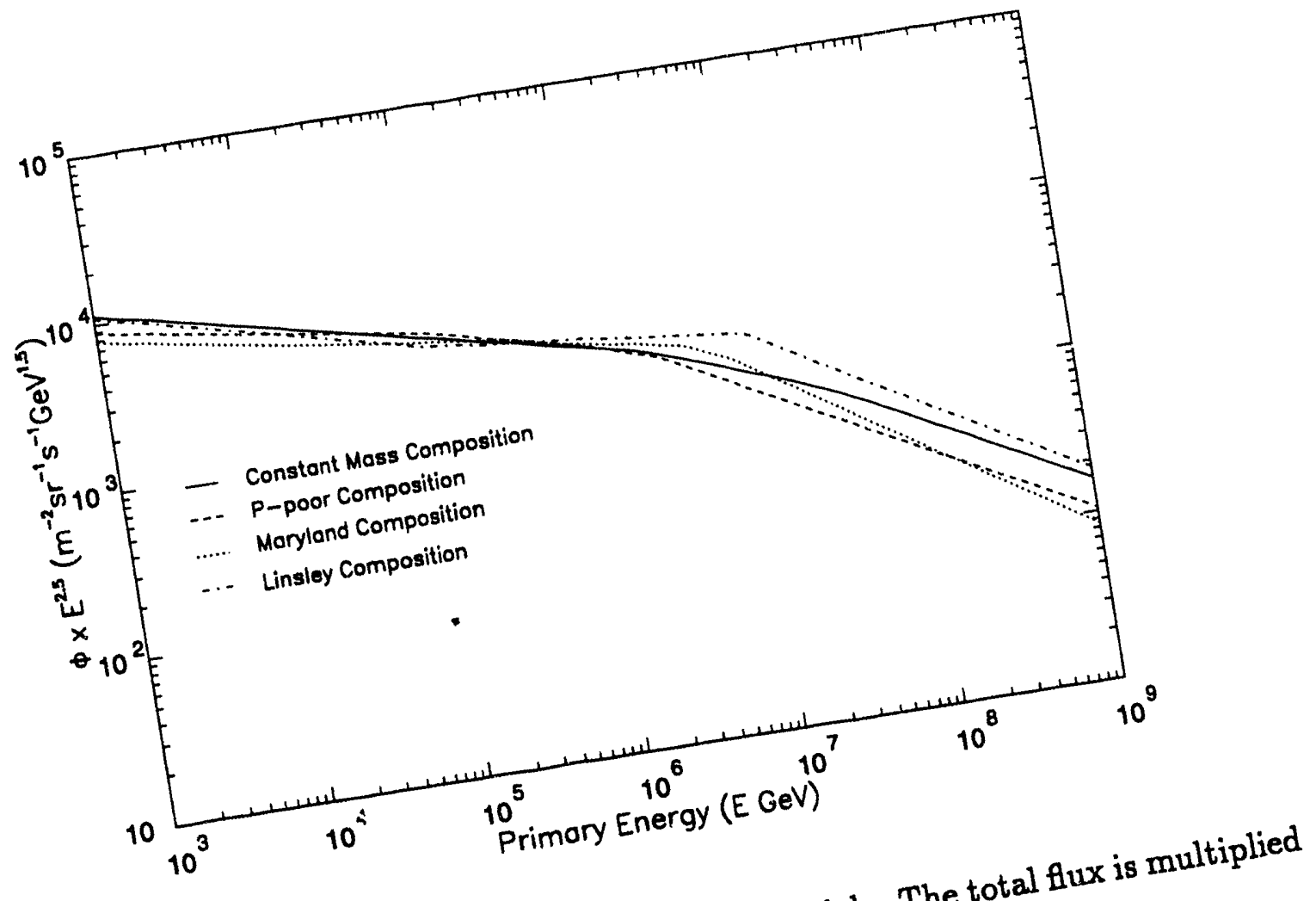
by $E^{2.5}$.

5.1.1 Primary Cosmic Ray

The primary cosmic ray energy sper

$$
\text { leading to muon bundles is given by }
$$

where $K$ is in nuclei $/ \mathrm{m}^{2} \mathrm{~s} s \mathrm{GeV} /$ nucleus). The parameters $K$ and $\gamma$ change when $E$ is greater than a cutoff value $E_{c}$ which the energy spectrum. Parameters $K$ and $\gamma$ andels considered here, the so-called model dependent. For the four compon in Fig. 5.1 for all four models. Slope changes all particle energy spectrum is shown in for the Linsley model (the dot-dash line in occur at two different primary energies for the $K$, slope $\gamma$, cutoff energy $E_{c}$ and slope above Fig. 5.1). The normalization factor $K$, so considered in our simulation, are given the cutoff energy for the four models to be considered in our sol 
in Table 5.1. Details of the four cosmic ray composition models are given in Section 5.2 .

The total integrated cosmic ray flux $N$, summed over all particles within the energy range $E_{\min }$ and $E_{\max }$, is given by

$$
N=\sum \frac{K}{\gamma}\left[E_{\max }^{\gamma}-E_{\min }^{\gamma}\right]
$$

Here $N$ is in nuclei $/ \mathrm{m}^{2}$ s sr. The total flux $N$ is calculated for two primary energy intervals, namely from $10 \mathrm{TeV}$ to $100 \mathrm{TeV}$ and from $100 \mathrm{TeV}$ to $10^{6} \mathrm{TeV}$. Different Monte Carlo runs were made for the two energy windows. Then the events generated within the second interval of primary energy were weighted according to the total flux within this energy window. In this way the full range of primary cosmic rays was sampled without consuming excessive amounts of computer time.

The minimum primary energy needed to produce at least a single muon on the 27 th level is $2 E_{\mu}^{\text {th }} \approx 1.6 \mathrm{TeV}$ for vertical muons. Since the rate of multiple muon events $\left(N_{\mu} \geq 2\right)$ below $10 \mathrm{TeV}$ is negligible, a minimum primary energy of $10 \mathrm{TeV}$ was used in the simulation.

\subsubsection{Muon Energy Losses in the Rock}

The rate of energy loss due to electromagnetic interactions of a muon with energy $E_{\mu}$ in $\mathrm{TeV}$, as a function of slant depth $y$, in $\mathrm{g} \mathrm{cm}^{-2}$, is given by [24]

$$
\frac{d E_{\mu}}{d y}=a+b E_{\mu}
$$

In this formula, $a$ is the rate of loss of energy through ionization and is taken to be constant for energy above a $\mathrm{TeV}$ :

$$
a=2.5 \times 10^{-6} \mathrm{TeV} / \mathrm{g} \mathrm{cm}^{-2} \text {. }
$$

The term $b E_{\mu}$ gives the energy loss due to bremsstrahlung. This loss increases with increasing energy. The constant $b$, appropriate for greenstone rock traversed by $\approx 5$ 
$\mathrm{TeV}$ muons, is taken to be

$$
b=4.0 \times 10^{-6} \mathrm{~g}^{-1} \mathrm{~cm}^{2} .
$$

For a flat overburden, the slant depth $y$ as function of the zenith angle $\theta$ is given by

$$
y=\rho D \sec \theta .
$$

At the Soudan site the average rock density obtained with ore samples taken throughout the mine $\rho=2.93 \mathrm{~g} \mathrm{~cm}^{-3}$ and the vertical depth $\mathrm{D}=723 \mathrm{~m}$. Eq. (5.3) can be integrated [27] over the distance from the surface to yield the threshold energy $E_{\mu}^{\text {th }}$ for a muon to reach the 27 th level:

$$
E_{\mu}^{\text {th }}=0.625\left(e^{4.0 \times 10^{-0} y}-1\right)
$$

Using this relation, the minimum energy required for a vertical muon to reach the 27th level is $0.85 \mathrm{TeV}$.

\subsubsection{Muon Bundle Multiplicity}

The work of Forti et al. [37] gives phenomenological formulas which are very useful in describing the average behavior of muon bundles underground. We now discuss the most important of these relations. The average number of muons, $\left\langle N_{\mu}\right\rangle$, produced by primary cosmic rays of nuclear mass number $A$, energy $E$, and zenith angle $\theta$ is given by [37]

$$
\left\langle N_{\mu}\right\rangle=\frac{A \sec \theta}{E_{\mu}} f\left(E_{p} / E_{\mu}\right) g\left(E_{p} / E_{\mu}\right),
$$

where $E_{p}$ is the primary energy per nucleon in $T e V$. The function

$$
g\left(E_{p} / E_{\mu}\right)=0.02126\left(E_{p} / E_{\mu}\right)^{0.7068}\left(1-E_{\mu} / E_{p}\right)
$$

describes the mean number of high energy muons in the atmospheric cascade; the function

$$
f\left(E_{p} / E_{\mu}\right)=\exp \left[\frac{48.27}{9.467+\left(E_{p} / E_{\mu}\right)^{3.330}}\right]
$$




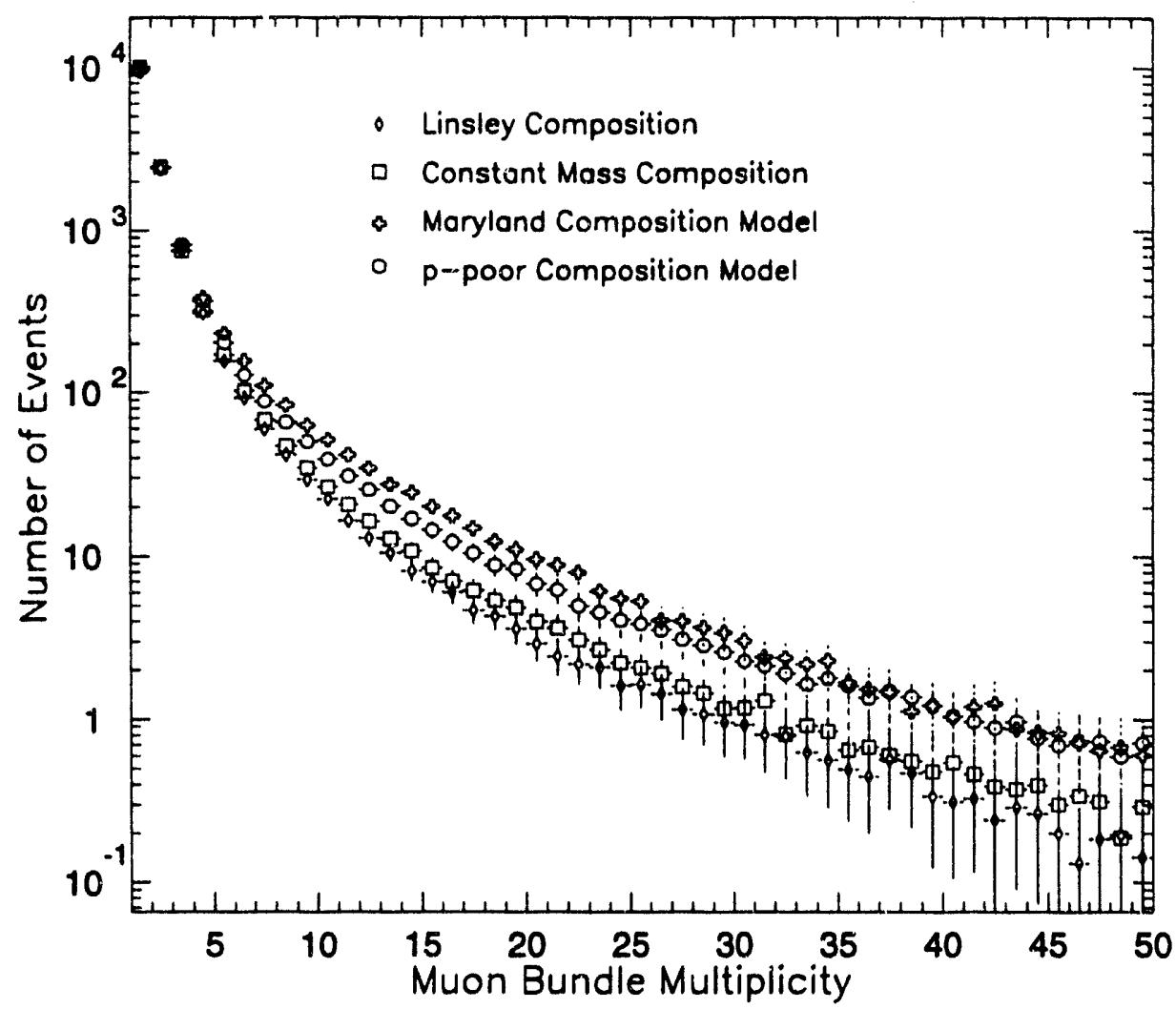

Figure 5.2: Muon multiplicity distributions from each of the four CR composition models.

is the correction factor for the mean number of muons at the depth of the detector. $f\left(E_{p} / E_{\mu}\right)$ approaches one for larger values of $E_{p} / E_{\mu}$. Equation (5.5) is valid for $E_{p} / E_{\mu}>2$ where $E_{\mu}$ is inferred from Eq. (5.3). The distribution of $N_{\mu}$ is described by a negative binomial function of the form

$$
P\left(N_{\mu}\right)=\left(\begin{array}{c}
N_{\mu}+k-1 \\
N_{\mu}
\end{array}\right)\left[\frac{\left\langle N_{\mu}\right\rangle / k}{1+\left\langle N_{\mu}\right\rangle / k}\right]^{N_{\mu}}\left[\frac{1}{1+\left\langle N_{\mu}\right\rangle / k}\right]^{k}
$$

with

$$
k=A^{2 / 3} 10^{F\left(\left\langle N_{\mu}\right\rangle / A\right)}
$$

and

$$
F\left(\left\langle N_{\mu}\right\rangle / A\right)=0.748+0.330 \log _{10}\left(\left\langle N_{\mu}\right\rangle / A\right)+0.045\left[\log _{10}\left(\left\langle N_{\mu}\right\rangle / A\right)\right]^{2} .
$$


In our Monte Carlo runs, the number of muons $N_{\mu}$ arriving at the $27 \mathrm{th}$ level are generated according to the Eq. (5.6). The multiplicity distributions obtained for the various models are given in Fig. 5.2. The distributions shown could only be observed in an underground detector of much larger dimensions than the existing detector and with perfect muon tracking.

\subsubsection{Separation of Muons from the Bundle Core}

The distribution of lateral distances of muons perpendicular to the shower axis is given by an inverse power law form:

$$
\frac{d N_{\mu}}{d R_{\mu}}=N_{\mu} \frac{(\alpha-1)(\alpha-2)}{R_{0}^{2-\alpha}} \frac{R_{\mu}}{\left(R_{0}+R_{\mu}\right)^{\alpha}} .
$$

The constant $R_{0}$ is related to the characteristic distance or mean separation $\left\langle R_{\mu}\right\rangle$ from the core and is given by

$$
R_{0}=\frac{\alpha-3}{2}\left\langle R_{\mu}\right\rangle
$$

where $\left\langle R_{\mu}\right\rangle$ is given by

$$
\left\langle R_{\mu}\right\rangle=G\left(E_{p}, E_{\mu}, \theta\right)\left[11.62 E_{\mu}^{-0.680}\left(E_{\mu} / E_{p}\right)^{0.114} \sec \theta\right]
$$

with

$$
\begin{gathered}
G\left(E_{p}, E_{\mu}, \theta\right)=A\left(E_{p}, \theta\right)+B\left(E_{p}, \theta\right)\left(E_{\mu}-1\right), \\
A\left(E_{p}, \theta\right)=1.39-0.383 X+6.72 \times 10^{-2} X^{2}+0.1(\sec \theta-1), \\
B\left(E_{p}, \theta\right)=\left[3.14 \times 10^{-2}+6.65 \times 10^{-3}(X-1)\right](2-\sec \theta)
\end{gathered}
$$

and with $X=\log _{10}\left(E_{p} / 1 \mathrm{TeV}\right)$. The parameter $\alpha$ is given by

$$
\alpha=C\left(E_{p}\right)\left[\frac{1.138}{-1.126+\left\langle R_{\mu}\right\rangle}+0.848\right]
$$

with

$$
C\left(E_{p}\right)=\exp \left(2.413-0.260 X+0.0266 X^{2}\right)
$$




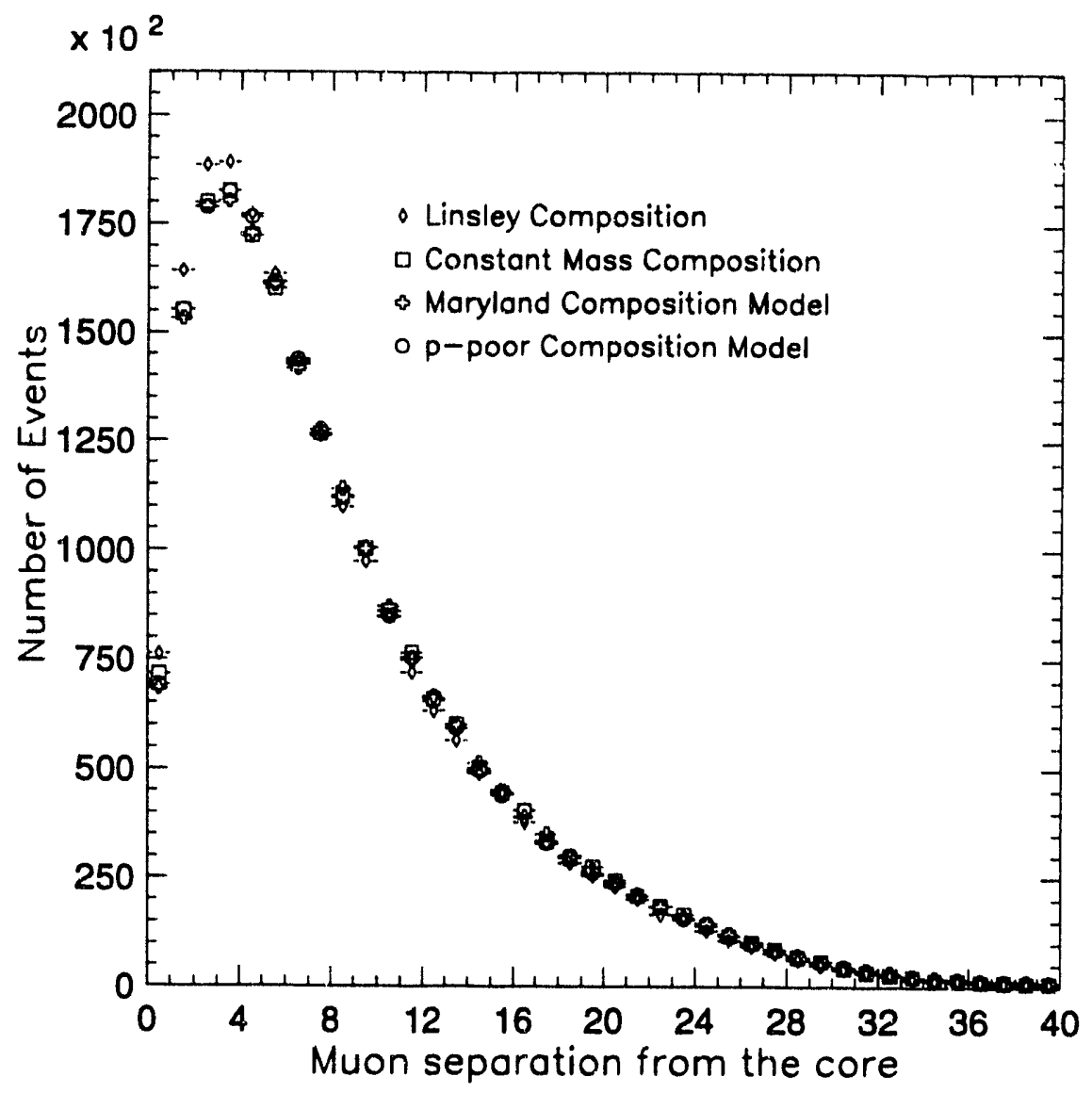

Figure 5.3: Lateral spread among bundle muons, measured from the bundle core, from the four models.

The mean separation $\left\langle R_{\mu}\right\rangle$ is in meters: the above formulas characterize bundles for which $\left\langle R_{\mu}\right\rangle>1.13 \mathrm{~m}$. If $\left\langle R_{\mu}\right\rangle<1.13 \mathrm{~m}$, the exponential form can be simplified to

$$
\frac{1}{N_{\mu}} \frac{d N_{\mu}}{d R_{\mu}}=\frac{4 R_{\mu}}{\left\langle R_{\mu}\right\rangle^{2}} e^{-2 R_{\mu} /\left\langle R_{\mu}\right\rangle} .
$$

Details concerning the relation between $\left\langle R_{\mu}\right\rangle$ and the transverse momentum $p_{t}$ are given in Chapter 6, Section 6.4. Distributions of $R_{\mu}$ for generated events, before detector cuts are applied, are shown in Fig. 5.3 for the four composition models.

\subsection{Models of Cosmic Ray Composition}

Four models for the primary cosmic ray nuclear composition are compared with our observations on muon bundles. The models are the P-poor model (PP), the Maryland model (MD), the Constant-Mass-Composition model (CMC), and the 
Linsley model (LI). The models can be divided into two categories. The Linsley model proposes that the cosmic rays consist mainly of protons. The CMC model predicts that the cosmic ray composition includes substantial amount of light elements such as protons, helium and the CNO group together with a few percent admixture of heavy elements such as $\mathrm{Si}, \mathrm{Mg}, \mathrm{Fe}$. These models are the "light" nuclear composition models. The Maryland and P-poor mociels are the "heavy" nuclear composition models, postulating a substantial contribution from iron and other heavy elements in the cosmic ray composition. A brief description of each model is given below.

\section{The Linsley Composition Model}

The composition model of Linsley [38] proposes that the percentage of protons in primary cosmic rays increases from $48 \%$ to $92 \%$ within the energy range from 1.0 $\mathrm{TeV}$ to $10^{6} \mathrm{TeV}$ (see Fig. 5.4 (a)). For this model, the spectral slope in the energy spectrum model changes in two places. Below the first cutoff energy of $100 \mathrm{TeV}$, the slope is 2.73; however it changes to 2.50 between $100 \mathrm{TeV}$ and $10^{4} \mathrm{TeV}$. The slope of the energy spectrum again changes beyond the second cutoff energy, this time to 3.23 .

In this model, the fraction of elements from $\mathrm{He}$ up to $\mathrm{Fe}$, is $12 \%$ to $16 \%$ in the low energy region and falls to $2 \%$ in the high energy region. It has been suggested that Active Galactic Nuclei may be sources of high fluxes of bigh energy protons [2]. The Linsley model is consistent with the scenario that the AGN contributes in an essential way to a predominance of protons in high energy cosmic rays [2].

\section{The Constant Mass Composition Model}

According to this model, the primary cosmic rays contain substantial amounts of both light and heavy elements [39]. For energies up to $2.0 \times 10^{3} \mathrm{TeV}$ the percentage of protons in this model is $36 \%$, while it is $19 \%$ for He, $13 \%$ for the CNO group, $19 \%$ for the $\mathrm{Mg}-\mathrm{Si}$, and $13 \%$ for iron. In the high energy region (above the "knee" 
), the percentage of protons falls to $\sim 24 \%$ while the amount of iron increases to $23 \%$. Similarly, the contents of the $\mathrm{Mg}-\mathrm{Si}$ group also increases to $26 \%$ (see Fig. 5.4 (b)). In the CMC model, unlike the other models, all the mass groups have the same spectral slope $\gamma$. Its value is 2.71 up to the energy where slope changes, and is 3.0 for higher energies.

\section{The Maryland Composition Model}

In the Maryland model, the proton flux is proposed to drop almost to zero in the high energy region above $10^{4} \mathrm{TeV}$. On the other hand, cosmic ray flux in this model is rich in heavy nuclei such as $\mathrm{Mg}-\mathrm{Si}$ and $\mathrm{Fe}$ [40] (see Fig. 5.4 (c)).

\section{The P-poor composition Mudel}

In the $\mathrm{P}$-poor model, the slope changes at energy $10^{5} \mathrm{TeV}$, which is low compared to the other models. The iron contribution in this model increases with increasing primary energy [41], while the proton content in the cosmic rays decreases with increasing energy (see Fig. 5.4 (d)). The fractions of other groups of elements such as $\mathrm{CNO}$ and $\mathrm{Mg}-\mathrm{Si}$ are fairly low compared to the Fe content in this model. (The plots in Fig. 5.4 were reproduced from Ref. [37] (Fig. 17.))

The parameters required for computer simulation of the models are given in Table 5.1. Normalization constants $K$, before the cutoff energy $\left(E_{c}\right)$, and slopes, before and after the cutoff energy, have been taken from Reference [37]. The normalization constants $K^{\prime}$, above the cutoff energy, have been calculated by equating the differential flux at $E_{c}$. Using these parameters, the integral fluxes have been calculated for the different compositions. These fluxes are the basis for generation of the primary energy in our simulatior.e leading to underground multiple muons at Soudan. Note that as mentioned earlier, in the Linsley model, the slope of the primary flux versus energy changes in two places for the proton component. 
Table 5.1: Normalization factors $K, K^{\prime}$, slopes $\gamma$, before and after cutoff energy $E_{c}$.

\begin{tabular}{|c|c|c|c|c|c|c|}
\hline \multirow{2}{*}{$\begin{array}{l}\text { Mass } \\
\text { Group }\end{array}$} & \multirow{2}{*}{$\begin{array}{c}\text { Mass } \\
\text { Number }\end{array}$} & $K\left(E<E_{c}\right)$ & $K^{\prime}\left(E>E_{c}\right)$ & \multicolumn{2}{|c|}{ Slope $\gamma$} & \multirow{2}{*}{$\begin{array}{c}E_{c} \\
\mathrm{GeV}\end{array}$} \\
\hline & & \multicolumn{2}{|c|}{$\mathrm{m}^{-2} \mathrm{~s}^{-1} \mathrm{sr}^{-1}(\mathrm{GeV} / \text { nucleus })^{-1}$} & $E<E_{c}$ & $E>E_{c}$ & \\
\hline \multicolumn{7}{|c|}{ P-poor Composition } \\
\hline$p$ & 1 & $1.51 \times 10^{4}$ & $4.78 \times 10^{5}$ & 2.70 & 3.00 & $1.0 \times 10^{5}$ \\
\hline $\mathrm{He}$ & 4 & $7.02 \times 10^{3}$ & $2.73 \times 10^{5}$ & 2.70 & 3.00 & $2.0 \times 10^{5}$ \\
\hline CNO & 16 & $2.68 \times 10^{3}$ & $5.10 \times 10^{5}$ & 2.61 & 3.00 & $7.0 \times 10^{5}$ \\
\hline $\mathrm{Mg} / \mathrm{Si}$ & 28 & $2.93 \times 10^{3}$ & $6.36 \times 10^{5}$ & 2.62 & 3.00 & $1.4 \times 10^{6}$ \\
\hline $\mathrm{Fe}$ & 56 & $8.56 \times 10^{2}$ & $1.43 \times 10^{6}$ & 2.50 & 3.00 & $2.8 \times 10^{6}$ \\
\hline \multicolumn{7}{|c|}{ Maryland Composition } \\
\hline$p$ & 1 & $1.98 \times 10^{4}$ & $3.83 \times 10^{7}$ & 2.75 & 3.35 & $3.0 \times 10^{5}$ \\
\hline $\mathrm{He}$ & 4 & $1.03 \times 10^{4}$ & $3.02 \times 10^{7}$ & 2.77 & 3.37 & $6.0 \times 10^{5}$ \\
\hline CNO & 16 & $2.15 \times 10^{3}$ & $1.34 \times 10^{7}$ & 2.60 & 3.20 & $2.1 \times 10^{6}$ \\
\hline $\mathrm{Mg} / \mathrm{Si}$ & 28 & $1.14 \times 10^{3}$ & $1.07 \times 10^{7}$ & 2.50 & 3.10 & $4.2 \times 10^{6}$ \\
\hline $\mathrm{Fe}$ & 56 & $5.95 \times 10^{2}$ & $8.19 \times 10^{6}$ & 2.50 & 3.10 & $8.4 \times 10^{6}$ \\
\hline \multicolumn{7}{|c|}{ Constant Mass Composition } \\
\hline$p$ & 1 & $1.72 \times 10^{4}$ & $1.16 \times 10^{6}$ & 2.71 & 3.00 & $2.0 \times 10^{6}$ \\
\hline $\mathrm{He}$ & 4 & $9.20 \times 10^{3}$ & $7.56 \times 10^{5}$ & 2.71 & 3.00 & $4.0 \times 10^{6}$ \\
\hline CNO & 16 & $6.20 \times 10^{3}$ & $7.32 \times 10^{5}$ & 2.71 & 3.00 & $1.4 \times 10^{7}$ \\
\hline $\mathrm{Mg} / \mathrm{Si}$ & 28 & $9.20 \times 10^{3}$ & $1.30 \times 10^{6}$ & 2.71 & 3.00 & $2.6 \times 10^{7}$ \\
\hline $\mathrm{Fe}$ & 56 & $6.20 \times 10^{3}$ & $1.07 \times 10^{6}$ & 2.71 & 3.00 & $5.2 \times 10^{7}$ \\
\hline \multicolumn{7}{|c|}{ Linsley Composition } \\
\hline$p$ & 1 & $2.60 \times 10^{4}$ & $1.84 \times 10^{3}$ & 2.73 & 2.50 & $1.0 \times 10^{5}$ \\
\hline $\mathrm{He}$ & 4 & $8.16 \times 10^{3}$ & $8.16 \times 10^{6}$ & 2.73 & 3.23 & $1.0 \times 10^{6}$ \\
\hline $\mathrm{CNO}$ & 16.0 & $5.65 \times 10^{3}$ & $1.06 \times 10^{7}$ & 2.73 & 3.23 & $3.5 \times 10^{6}$ \\
\hline $\mathrm{Mg} / \mathrm{Si}$ & 28 & $7.30 \times 10^{3}$ & $1.93 \times 10^{7}$ & 2.73 & 3.23 & $7.0 \times 10^{6}$ \\
\hline$p$ & 1 & $1.84 \times 10^{3}$ & $8.03 \times 10^{6}$ & 2.50 & 3.02 & $1.0 \times 10^{7}$ \\
\hline $\mathrm{Fe}$ & 56 & $6.33 \times 10^{3}$ & $2.2 \times 10^{7}$ & 2.73 & 3.23 & $1.3 \times 10^{7}$ \\
\hline
\end{tabular}



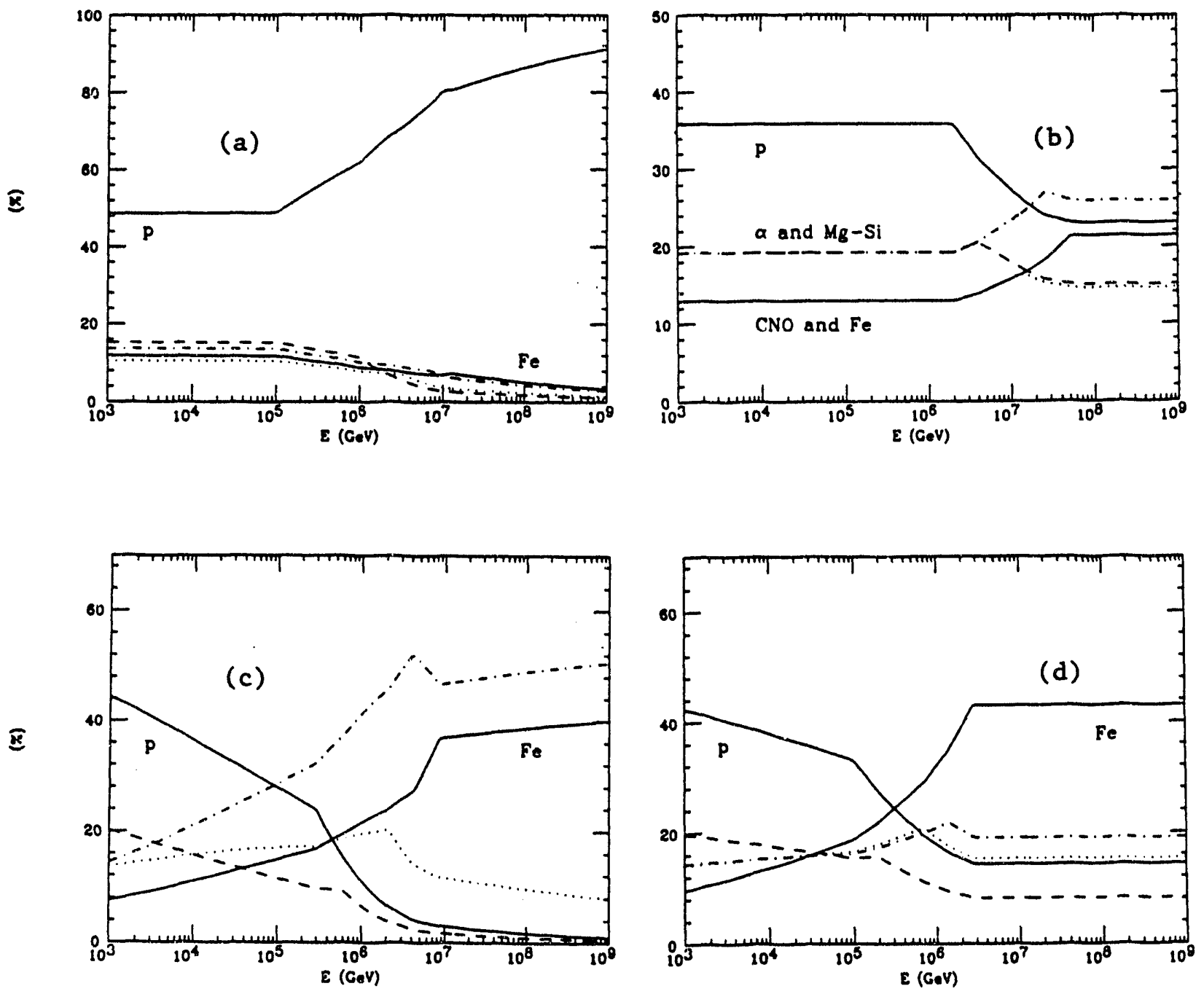

Figure 5.4: Fractional composition versus primary energy for composition models (a) Linsley, (b) CMC, (c) Maryland, and (d) P-poor. The solid curves in all figures give the contributions of $p$ and $\mathrm{Fe}$. Contributions from alpha particles, from the $\mathrm{CNO}$ group, and from the intermediate mass nuclei $\mathrm{Mg}$ and $\mathrm{Si}$, are depicted by dashed, dotted, and dot-dashed curves respectively. 


\subsection{Uncertainties in the Monte Carlo Simulation}

Since our Monte Carlo simulation is based upon many assumptions and approximations, predictions from the simulation contain systematic errors. There are three major contributors to such errors in our simulation:

(i) Topology of the earth overburden above the detector.

(ii) The detector geometry and its efficiency.

(iii) The hadronic interaction model which has been used to derive essential equations such as those used to calculate $\left\langle N_{\mu}\right\rangle$ and $R_{\mu}$.

\subsubsection{Earth overburden above the detector}

The surface of the earth above the detector is not flat, rather it is a wedgeshaped hill (see illustration in Fig. 4.5). There is a long ridge running along the east-west direction, which gives more overburden in that direction. Along the northsouth direction, the slope changes quickly and the overburden falls off (see section 4.3). The slant depth, which is defined by the polar angles $\theta$ (zenith angle) and $\phi$ (azimuthal angle), is calculated using a detailed topographical map of the hill [36] corresponding to one degree zenith angle binning and one degree azimuthal angle binning.

The slant depth for a muon path also depends on the rock density according $y=\rho D \sec \theta$. The average rock density is calculated from sampling of rock taken from various places near the Soudan 2 site [43]. An average density of $2.93 \mathrm{~g} \mathrm{~cm}^{-3}$ was deduced from the above sampling, to be compared to the Standard Rock density which is $2.55 \pm 0.05 \mathrm{~g} \mathrm{~cm}^{-3}$ [7].

Uncertainties in the density of the rock and in the slant depth calculation can give rise to systematic error in the Monte Carlo simulation. To study the sensitivity of the simulation to change in the rock density, three different sets of simulated events have been produced using different density values, namely $2.55,2.65$ and 2.93 
$\mathrm{g} \mathrm{cm}^{-3}$. The absolute event rates have been compared with the data as discussed in Chapter 6.

In addition to the rock density, the average values for atomic number $(Z)$ and atomic mass number $(A)$ for Soudan rock can also contribute to systematic error since muon energy loss in passage through the rock depends upon $Z$ and $A$. Since energy loss due to ionization is proportional to $Z / A$, and since energy loss due to the radiative processes such as bremsstrahlung is proportional to $Z^{2}[44]$, it is necessary to get a good estimate for average $Z$ and $A$ as well as for the density of the rock, in order to calculate the constants $a$ and $b$ in Eq. (5.3) (see Section 6.4 for more detail).

\subsubsection{Detector geometry and geometric efficiency}

A uniform rectangular parallelepiped was assumed to describe the central detector in our simulation. The central detector has cracks along the inter-module boundaries both in the east-west direction and north-south direction; these were not included in the simulation. The veto shield surface also had various cracks and holes when the data were taken for this analysis. The irregularities in the shield configuration have been included into the shield geometry in the simulations.

We introduced a veto shield single layer electronic efficiency, which is $95 \%$, to simulate the recording of muon crossings by the shield manifolds. Our selection of muon tracks is fully determined by the veto shield hit pattern, and the veto shield efficiencies are included in the Monte Carlo. A comparison of data with the Monte Carlo simulations is given in Chapter 6.

\subsubsection{Hadronic interaction model for cosmic rays}

Assumptions and simpifications have been made in the hadronic interaction model for the primary cosmic ray - air ( $\mathrm{N}$ and $\mathrm{O}$ ) interaction. Those which have 
been investigated by Forti et al. [37] are summarized below.

(1) Inelastic cross-sections: Cross-sections for proton-air collisions at very high energies (over $1000 \mathrm{TeV}$ incident) have been obtained by extrapolation from existing accelerator data. To explore sensitivity of underground muon properties to these cross-sections, two values of $\sigma_{p \text {-air }}, 416 \mathrm{mb}$ and $382 \mathrm{mb}$, have been tried in Monte Carlo simulation at $1000 \mathrm{TeV}$. It was found that the smaller cross-section resulted in a $5 \%$ reduction in the mean number of muons predicted at the MACRO depth.

(2) Associated production and prompt muon production: Associated production of hyperons and kaons has not been explicitly included in the calculations of Ref. [37]. With $\Lambda^{0}$ production included, a two percent increase in the mean number of muons per shower induced by $1000 \mathrm{TeV}$ primary cosmic rays was obtained. The production of prompt muons originating with production and decay of charm and bottom mesons is expected to be important at depths over 7000 mwe, and was neglected here.

(3) Nuclear target effects (differences between nucleon-nucleus and nucleon-nucleon collisions): Turning the nuclear target effects completely off produced only a small percentage change in the muon bundle properties. We conclude that any contribution of the model of nuclear target effects to our systematic errors is negligible.

(4) Inelasticity versus primary energy: Inelasticity in the high energy hadronic interaction model is also model-dependent and energy-dependent. Three interaction models were tested to explore uncirtainties in the muon properties. The muon flux at MACRO depth varies by $7-12 \%$ in the models considered. No model dependence is reported in Ref. [37] for the lateral distributions.

(5) Diffractive interactions: Full Monte Carlc simulations were tried both with and without a diffractive component in the hadronic interactions. Changes in underground muon multiplicity and in lateral distributions at the MACRO depth were found to be less than $5 \%$ and $10 \%$, respectively. The contribution of any uncertainties in the treatment of diffraction in the simulation to our systematic errors is 
therefore negligible.

(6) Fragmentation of the primary nucleus: Ir, se superposition model, a cosmic ray shower with a nucleus of atomic mass number $A$ with energy $E$ is treated as equivalent to $A$ individual showers by $A$ nucleons with energy $E / A$ associated with each nucleon. Such a picture is of course a very crude characterization of the actual hadronic interaction between primary cosmic rays and the atmosphere. To examine the sensitivity of the underground muon distributions to the simplifications inherent with the superposition model, a fragmentation model was also tried. In the latter model, the primary nucleus fragments only partially in the first interaction and then undergoes further collisions; thus its overall hadronic shower cascade develops relatively further down in the atmosphere. As a result, both the mean number of muons underground and their mean distance from the shower core are predicted to be lower than those obtained with the superposition model. The muon multiplicity rates for 3-muon events and for 16-muon events were found to be lowered by $3 \%$ and $12 \%$ respectively for the fragmentation model compared to the superposition model, when the light composition model (the Linsley model) was considered for the simulation. For a heavy composition model (the Maryland model), the above reduction is $9 \%$ and $21 \%$ for 3 - and 16-muon events, respectively. The changes in multiplicity did not exhibit a dependence on primary energy, zenith angle, or detector depth. Similarly, a reduction in the lateral muon displacement when compared to the superposition model was found to be independent of zenith angle and detector depth, but not of primary energy. The magnitude of this reduction was $20 \%$ at 1 $\mathrm{TeV} /$ nucleon, and decreasing with increasing primary energy.

An interaction model called "the semi-superposition model" has been proposed recently by J. Engel et al. [45]. In this extension of the superposition model, a realistic distribution for the first interaction points of cosmic ray nuclei wit: air nuclei is used. Here, a cosmic ray nucleus is bypothesized to interact immediately when it first enters the atmosphere. Only a fragment nucleus with the "wounded" 
nucleons (that took part in the interaction) removed continues on until all nucleons from the initial nucleus interact with the atmosphere. For the cosmic ray - air interaction, this leads to larger fluctuations in hadronic showers generated by the semi-superposition model than in showers generated using the superposition model. For example, for an incoming iron nucleus of $5600 \mathrm{TeV}$, the multiplicity distribution of muons with energies above $1 \mathrm{TeV}$ would have the same $\left\langle N_{\mu}\right\rangle$, but its width would be larger by $25 \%$ with the semi-superposition model. No effect is expected in the muon lateral distribution.

A crude estimation has been made to evaluate overall systematic variation in the properties of underground muon bundle due to the interaction model used for Monte Carlo simulation. We take a quadrature sum of the individual errors given in each category discussed above. For light composition models, we estimate the error in mean multiplicity to be $8 \%$ for low multiplicities and $14 \%$ for high multiplicities. For heavy composition models, we estimate the uncertainty in mean multiplicity to be $12 \%$ for low multiplicities and $22 \%$ for high multiplicities.

In Forti et al. [37], all the uncertainties quoted are for MACRO depths, between 3000 mwe and 5000 mwe. For Soudan's depth which is 2090 mwe, these uncertainties would be presumably smaller. 


\section{Chapter 6 \\ Properties of Multiple Muon Events At Soudan}

Multiple muon events recorded in the May through July 1991 running of Soudan 2 are compared with simulated events generated using four different cosmic ray composition models. In these simulations, we have attempted to include the effects of detector acceptance in a realistic way. Subsequent to the selection and reconstruction of muon tracks as discissed in Chapter 3, additional cuts involving muon zenith angles and track lengths are applied to the real events and to the simulated data. Losses originating with inefficiencies and limited resolution of the shield are also applied to the simulated data. After all cuts, the final multiple muon sample used for analysis contains 54,035 events.

As described in Chapter 5, there are four different models for the nuclear composition of the primary cosmic ray flux which are frequently discussed in the literature [37]. These are (i) the Linsley model, which proposes a light nuclear composition; (ii) the Constant Mass Composition model, which also favors a light composition with small percentages of heavy nuclei; (iii) the Maryland model, which proposes a relatively heavy nuclear composition; and (iv) the $\mathrm{P}$-poor model which also favors a heavy composition. These four models are the basis for different Monte Carlo simulations of muon bundle detection underground on Soudan's level 27.

\subsection{Additional Cuts to the Data}

As discussed in Chapter 4, the central tracking calorimeter, which provides the event trigger, has good acceptance in most directiors. However, the detection 


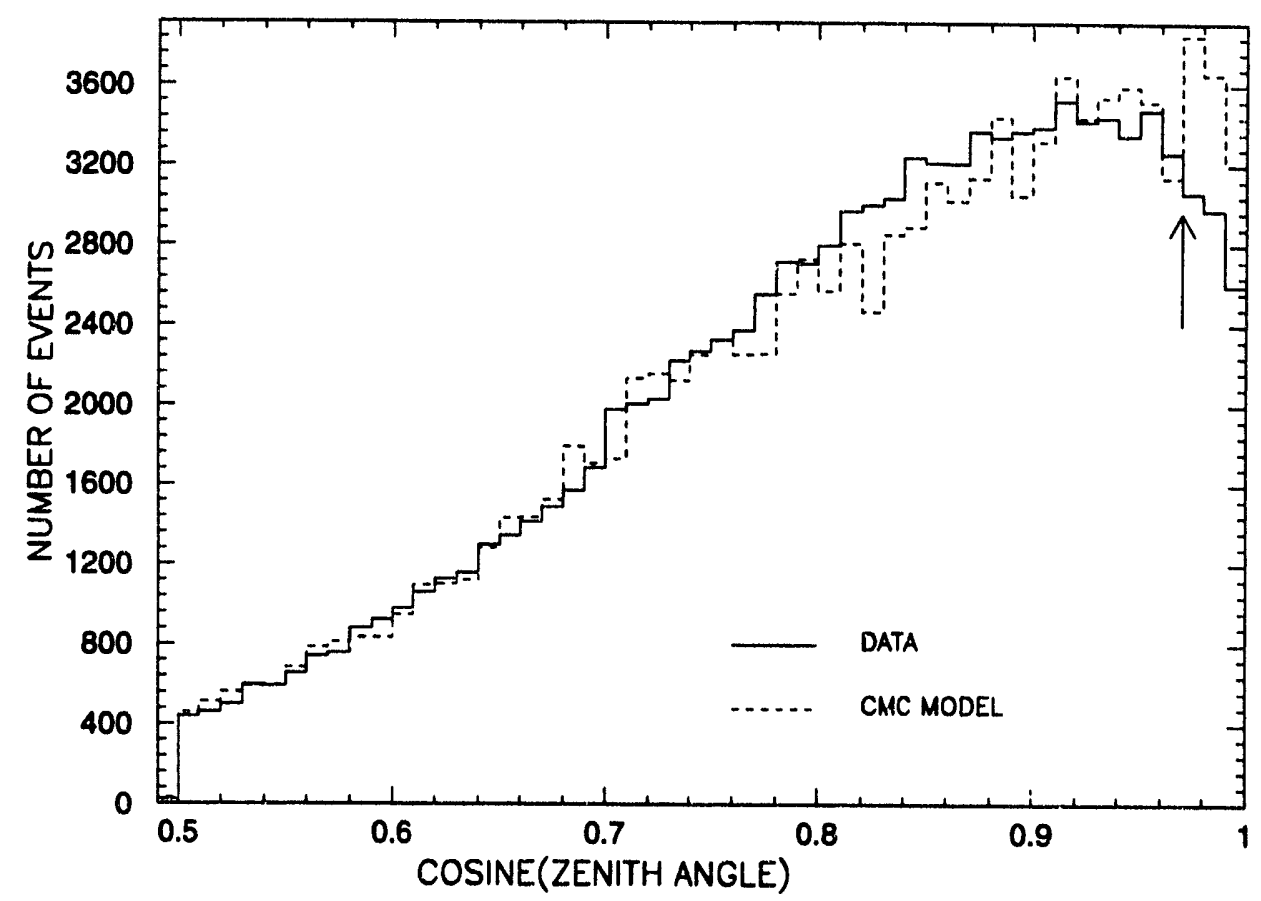

Figure 6.1: The zenith angle distribution, corspared to the CMC model. The arrow indicates where the zenith angle cut has been made. The central detector triggering inefficiency is clearly seen between $0^{\circ}$ and $15^{\circ}$.

efficiencies for muon tracks along the nortb-south and east-west directions are reduced due to the gaps between the central detector modules and due to the trigger requirements. Losses of muons from these directions can be seen in the zenith angle and the azimuthal angle distributions (see Figs. 4.3 and 4.4). Detection of muons incident along the vertical is also poor compared to other directions. In the case of vertical muons, relatively few anode wires pick up ionization and the time differences between successive edges are nearly zero (differences greater than $0.6 \mu$ s are needed for triggering). Consequently vertical charged tracks seldom satisfy the trigger requirement (see section 2.6). To avoid uncertainties from these effects, cuts have been applied to the zenith angle and to the azimuthal angle in both the real and simulated event samples. The zenith angie $(\theta)$ distribution $\left(0^{\circ}<\theta \leq 60^{\circ}\right)$ for the data and for 
the CMC model is shown in Fig. 6.1. Between $0^{\circ}$ and $15^{\circ}$ the difference between the model and the data, which reflects the trigger problem along the vertical direction, is clearly seen. As a result we have restricted the zenith angle range to be from $15^{\circ}$ to $60^{\circ}$ in the analysis reported here. The upper limit of the zenith angle of $60^{\circ}$ has been set because of limited event statistics at large zenith and because of of limitations of the Monte Carlo simulations [37]. The allowed ranges for azimuthal angle have been restricted to $8^{\circ}-82^{\circ}, 98^{\circ}-172^{\circ}, 188^{\circ}-262^{\circ}$ and $278^{\circ}-352^{\circ}$ in order to eliminate the acceptance holes along the north-south and east-west directions.

We require muon tracks which are reconstructed using only the central detector to have length greater than $1.0 \mathrm{~m}$ within the central detector volume. For tracks which are reconstructed using only hits in the veto shield, we require that distances between the defining hits exceed $2.0 \mathrm{~m}$. These cuts are designed to eliminate difficult track reconstruction situations involving hits around edges and corners of the central detector and of the shield. If any two tracks within the central detector are closer than $8 \mathrm{~cm}$, the two tracks are taken to be one track. (Relatively few muons closer than $8 \mathrm{~cm}$ are detected, and the CMC model predicts low rates for such muon pairs (see Fig. 6.2).) In cases of pairs of tracks where one track is reconstructed from the central detector and other track from the shield (outside of the central detector), or where both tracks are reconstructed from the shield, the minimum acceptable perpendicular distance between the tracks is taken to be $30 \mathrm{~cm}$. Although the spatial resolution of the central detector is nearly $1.0 \mathrm{~cm}$ in all three spatial coordinates, the overall resolution for separation of tracks in this analysis is nearly one and a half shield module widths which is $30 \mathrm{~cm}$. The same selection methods and cuts are applied to muon hits generated via Monte Carlo for all four models, enabling us to compare the data and models under similar conditions. Table 6.1 gives the number of muon tracks which have been used in the analysis of this Thesis. 


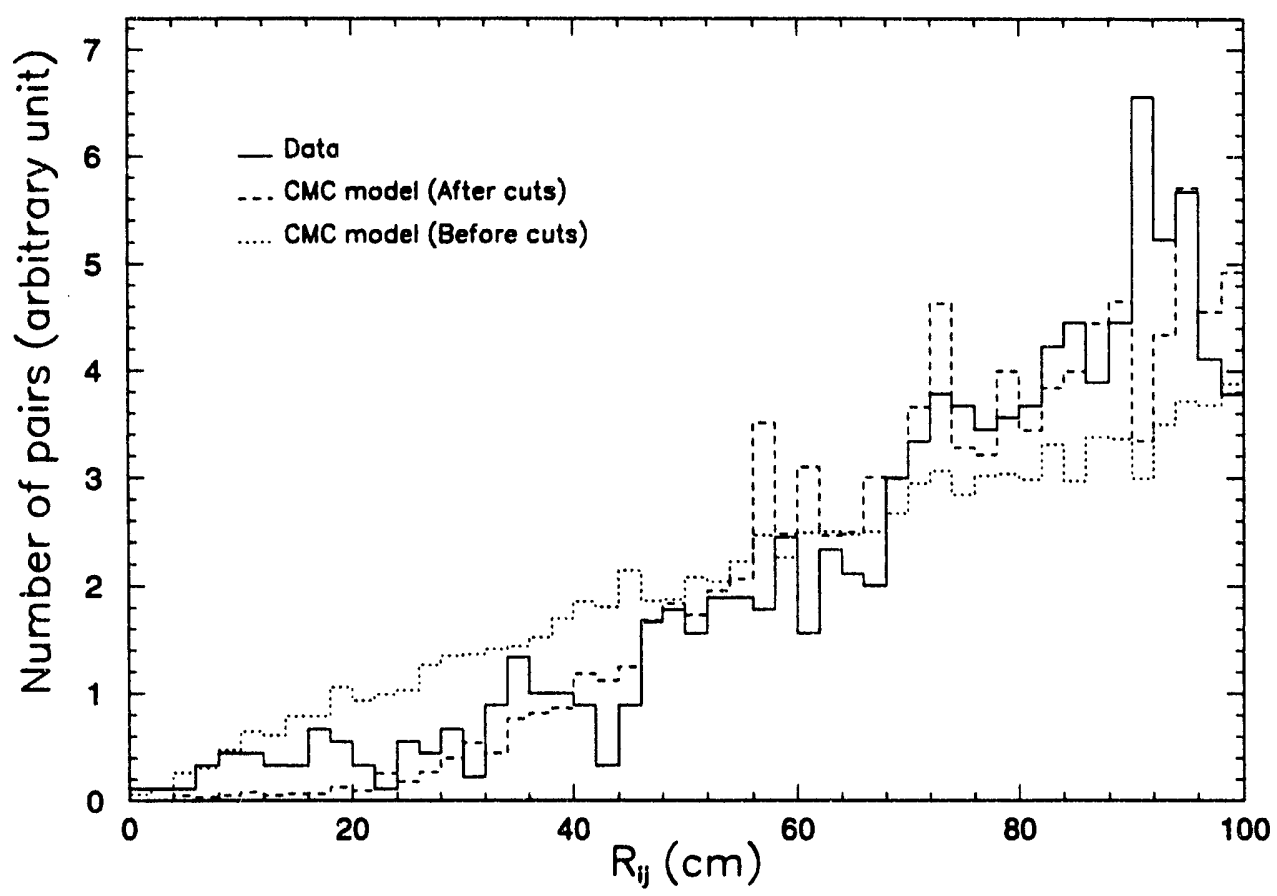

Figure 6.2: Intermuon separation recorded in the central detector, as observed in the data and as predicted by the CMC model.

\subsection{Track Categories by Reconstruction}

Reconstructed muon tracks can be assigned to one of four different categories as given in Table 3.2: (1) A muon track which has four-layer coincident hits is in the first category. Such a track has adjacent group hits at both entering and exiting points on the veto shield. A muon track may pass through the overlapped region between the east and west ceilings, in which case it might have five or sixlayer coincidence. A track of this type is also assigned to the first category. Tracks which have three-layer coincid nt hits are assigned to the second category. These tracks contain adjacent groups on one shield surface and in-time singles on an opposing surface. (3) The muon tracks of the third category have one of three different combinations of hits. These tracks all have hits in the central detector. 
Table 6.1: Multiple muon events in the 1991 data.

\begin{tabular}{|l|c|c|c|}
\hline \multicolumn{4}{|c|}{ Muon Sample } \\
\hline Before cuts (75,332 events) \\
\hline Multiple & Central & \multicolumn{2}{|c|}{ Shield } \\
\cline { 3 - 4 } Muons $\left(N_{\mu} \geq 2\right)$ & Detector & $M_{\perp}$ & $M_{\|}$ \\
\hline Number of tracks & 108,730 & 64,980 & 20,965 \\
\hline \hline After cuts $(54,035$ events) \\
\hline Multiple & Central & Shield \\
\cline { 3 - 4 } Muons $\left(N_{\mu} \geq 2\right)$ & Detector & $M_{\perp}$ & $M_{\|}$ \\
\hline Number of tracks & 73,817 & 45,848 & 16,369 \\
\hline \hline
\end{tabular}

(3a) In one possible combination there are hits in two layers of the veto shield, one layer on one side of the shield and an other layer on the other side. These are single layer hits which are in-time. (3b) A second combination is with an adjacent group on one side and with a gap on the other side. Unfortunately, when this data sample was taken, the floor panels contained numerous gaps. (3c) The third combination is similar to the second, however in this type, one end of the track points to a shield panel which does not register a hit, presumably due to electronic inefficiency of the shield. The latter tracks could originate with through-going muons or could be due to muons which range to stopping in the central detector. (4) In the fourth track category, a trajectory has only a single hit on one shield surface. The other end of the track may be in a gap or in a shield manifold which failed to respond.

The above four categories of reconstructed tracks are tallied separately and shown in Figs. 6.3(a) and 6.3(b). The Constant Mass Composition model has been used here to provide a comparison with the data. Fig. 6.3(a) shovs the distribution of the number of layers responding for all categories of muon tracks above. An additional selection is made for the muon tracks tallied in Fig. 6.3(b). Here we eliminate tracks which are reconstructed using only single layer hits, and tracks which have a double 


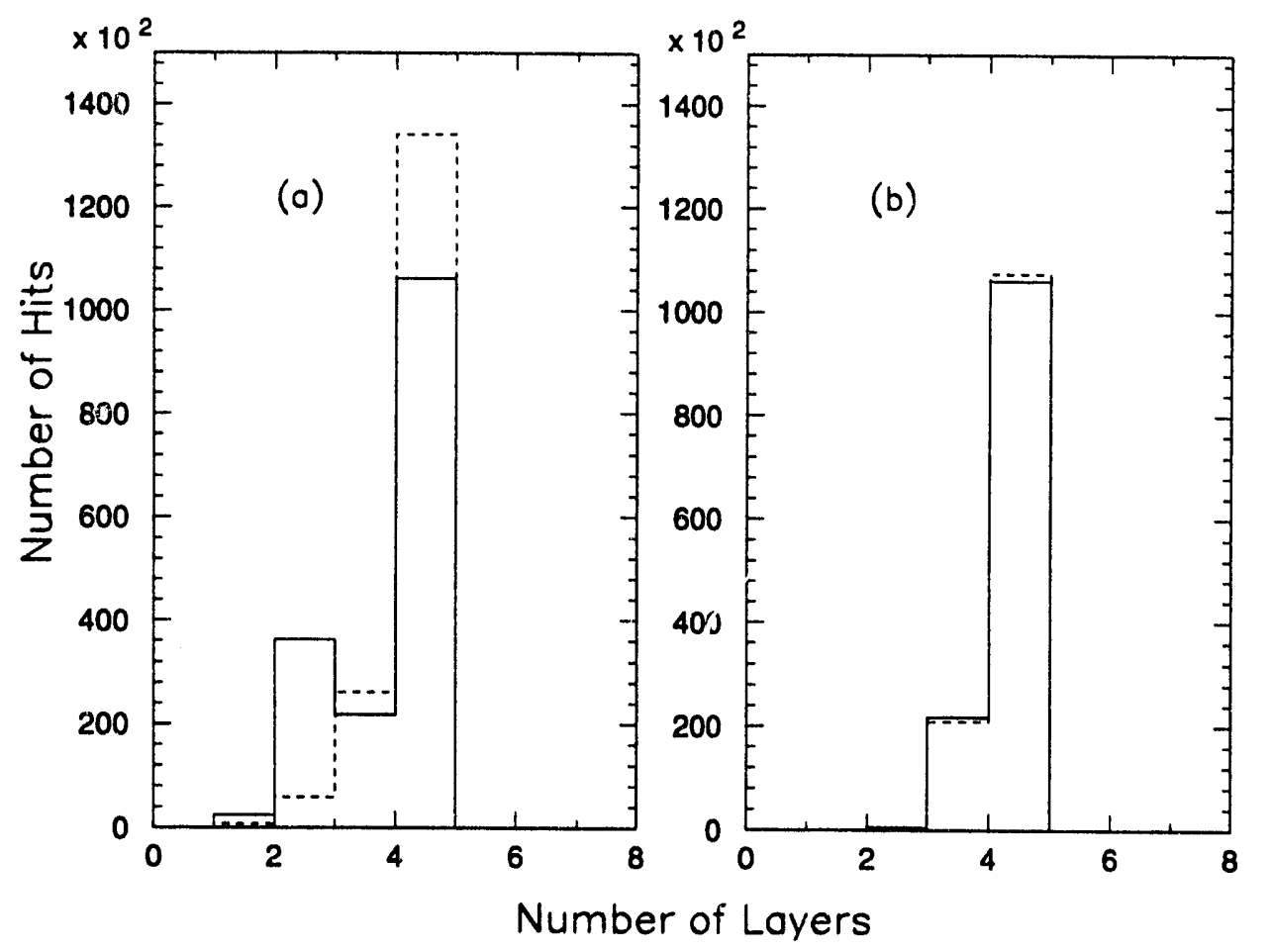

Figure 6.3: Number of hits versus the number of shield layers responding to a track, for the data and for the simulation (solid and dashed histograms respectively). In Fig. 6.3(a), the two layer hits with no hits on one end are included.

layer hit on one shield surface but with no response on the opposing surface. In the simulation used for this comparison, the shield efficiency per layer is taken to be $95 \%$. (A calculation based upon the distribution of hit combinations in the veto shield yields a single layer detection efficiency of $95.6 \%$. See Chapter 4.)

The comparison in Fig. 6.3(b) is a stringent test of the accuracy of the representation of shield response in our simulations, and it is pleasing that the distributions match up so well. In Fig. 6.3(a) we see that tracks which have double layer hits on one shield surface are distinctly more abundant than predicted via the simulation. We ascribe the difference to result from muon tracks which are relatively slow and either range to stopping within the central detector or undergo large-angle deflection; these effects are not included in our muon bundle simulations, which treat muons as straight, through-going tracks. 


\subsection{Muon Bundle Multiplicity Distribution}

The distribution of the number of muons $N_{\mu}$ within muon bundles can be used to study the primary composition of cosmic rays because the multiplicity depends on the atomic number $A$ of the primary particle [1]. A plausible dependence of $N_{\mu}$ on $A$ is indicated in Eq. (5.5). In principle, the observed muon multiplicity distribution can be compared with Monte Carlo expectations for a given nuclear composition and energy spectrum to determine the composition of the cosmic ray flux. Comparison of our observed multiplicity distribution with the multiplicity distributions obtained using the four different composition models is shown in Fig. 6.4(a), (b), (c) and (d). The average multiplicity $\left\langle N_{\mu}\right\rangle$ is $2.51 \pm 0.01$ for multiple muon events with two or more than two muons in a muon bundle.

Fig. 6.5 shows the multiplicity distribution for shield tracks and for the selected tracks in the central detector (open circles), together with the estimated number of true through-going muon tracks (open squares) which are removed by our cut of the Hits/No hits category of tracks from CLASS 1. (See Section 4.2.) In Fig. 6.5 it can be seen that correction for this loss of muons gives rise to a systematic increase in muon multiplicity in every bin of about 0.5 standard deviations. 

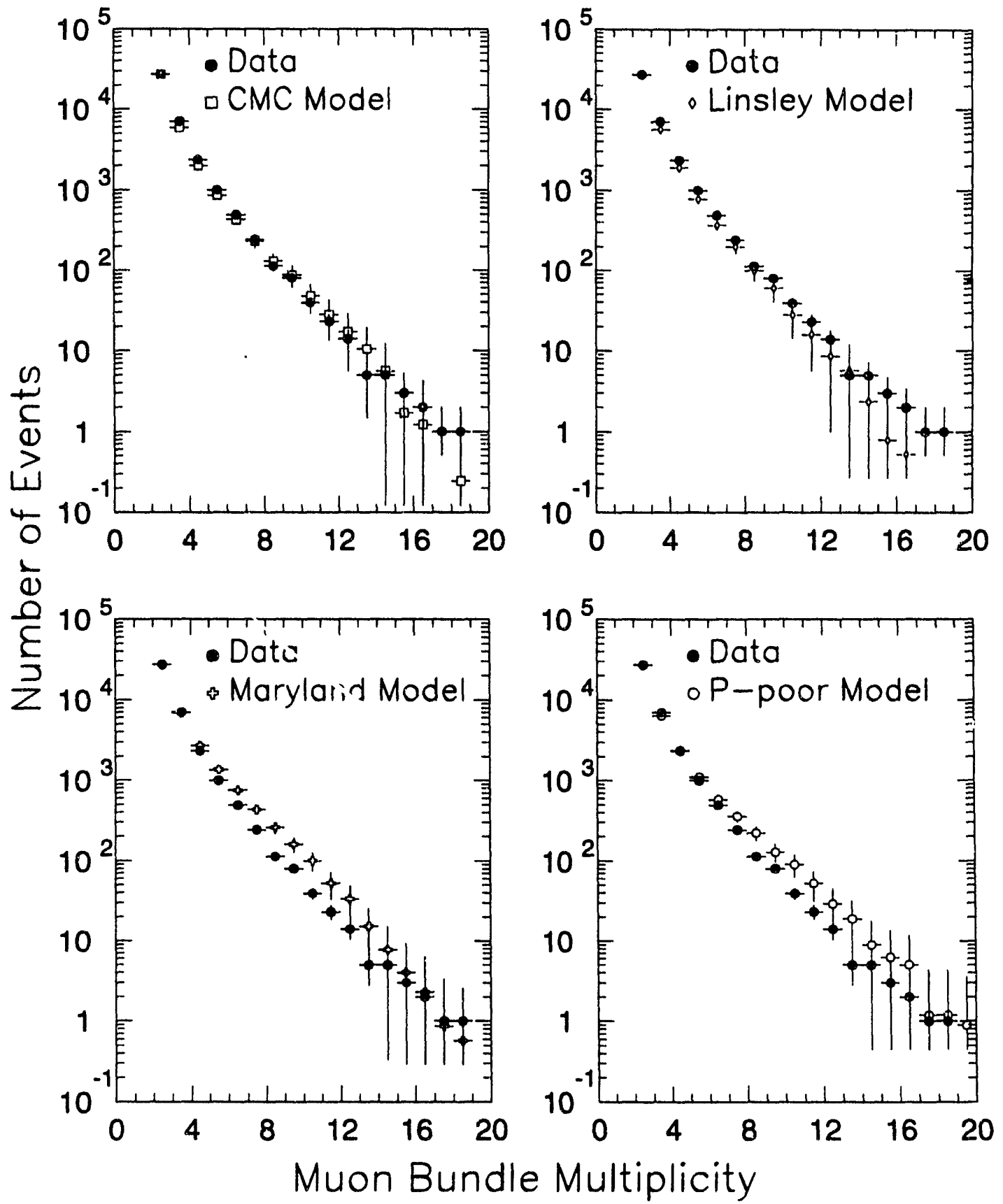

Figure 6.4: Distribution of muon bundle multiplicity, compared to expectations of four cosmic ray composition models. 


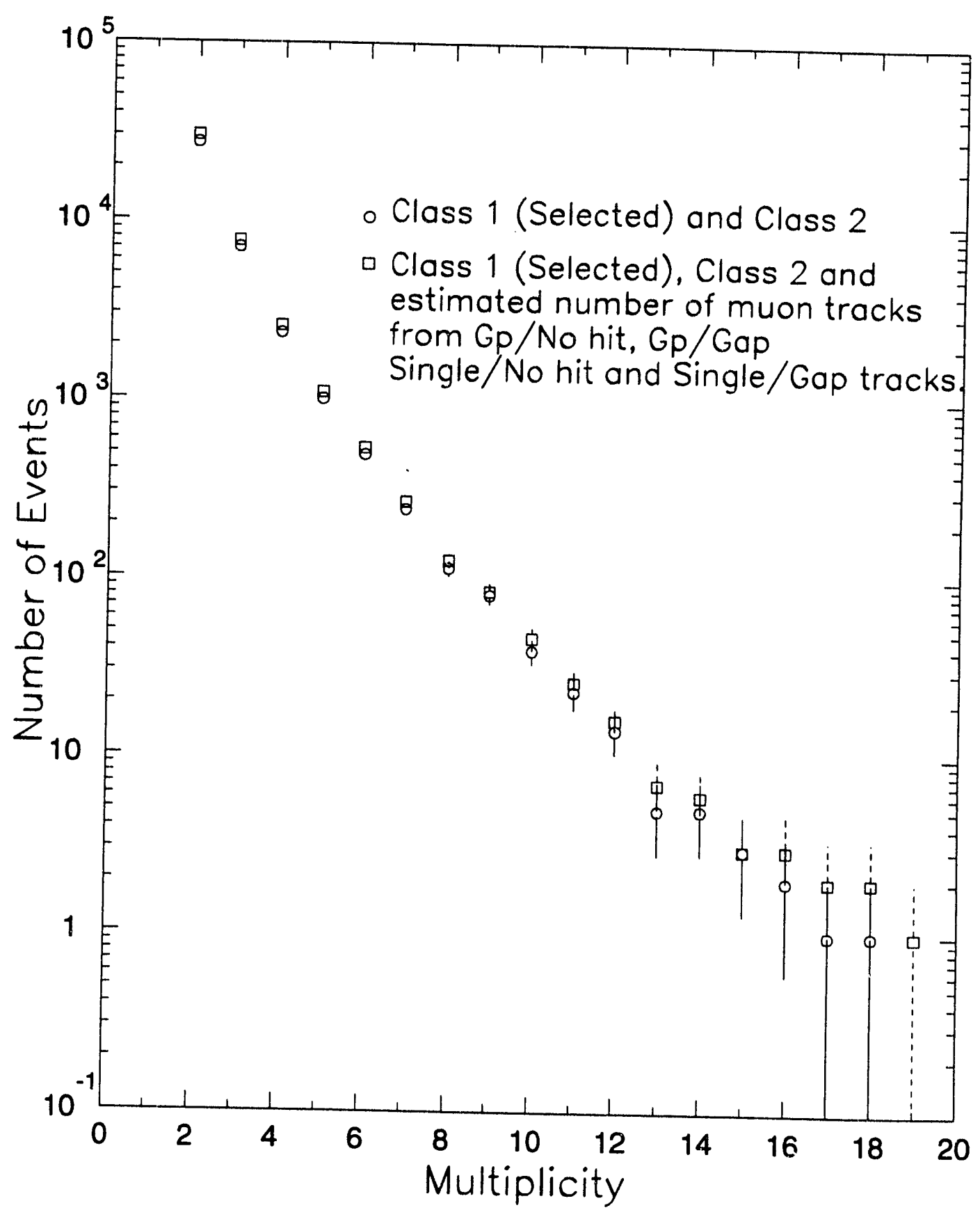

Figure 6.5: Distribution of muon bundle multiplicity, compared to estimated total multiplicity which includes some of the tracks from Group/No hit, Group/Single, Single/No hit and Single/Gap categories. 
In Fig. 6.4, events for all four models have been normalized to the number of twomuon events in the data, consequently it is the shapes of the distributions which are compared. The solid circles show the data points while open symbols show predictions of the models. The "light" nuclear composition models (the Linsley composition model and the CMC model) show somewhat better agreement with the experimental data. The Maryland and P-poor composition models, which propose that the jrimary cosmic rays contain large fluxes of heavier nuclei up to Fe, predict multiplicity distributions which are broader than those observed. We note, however, that correction for the Hits/No-hits track category (Fig. 6.5) lessens the discrepancy between the heavy models and the data.

The relationship between muon bundle multiplicity $N_{\mu}$ and the nucleon number $A$ of a primary cosmic ray nucleus involves three considerations:

\section{(i) The rock overburden:}

For underground experiments, the presence of the rock overburden implies a threshold energy for a muon to reach the detector. For either proton or iron primaries, the muon yield at Soudan 2, calculated using Eq. (5.5), is shown in Fig. 6.6. In the case of $\mathrm{Fe}$ cosmic rays, a primary energy greater than $100 \mathrm{TeV}$ is required in order to produce secondary muons which can reach our detector. For primary energies beyond $\sim 400 \mathrm{TeV}$, the muon yield from Fe primaries exceeds the yield from proton primaries.

(ii) The superposition model for nucleus - nucleus collisions:

In this model, an iron primary with energy $E$ breaks up and produces 56 individual air showers, one shower per nucleon, each with energy $E / 56$. The following simplified equation [1] illustrates the above two points concerning the number of 


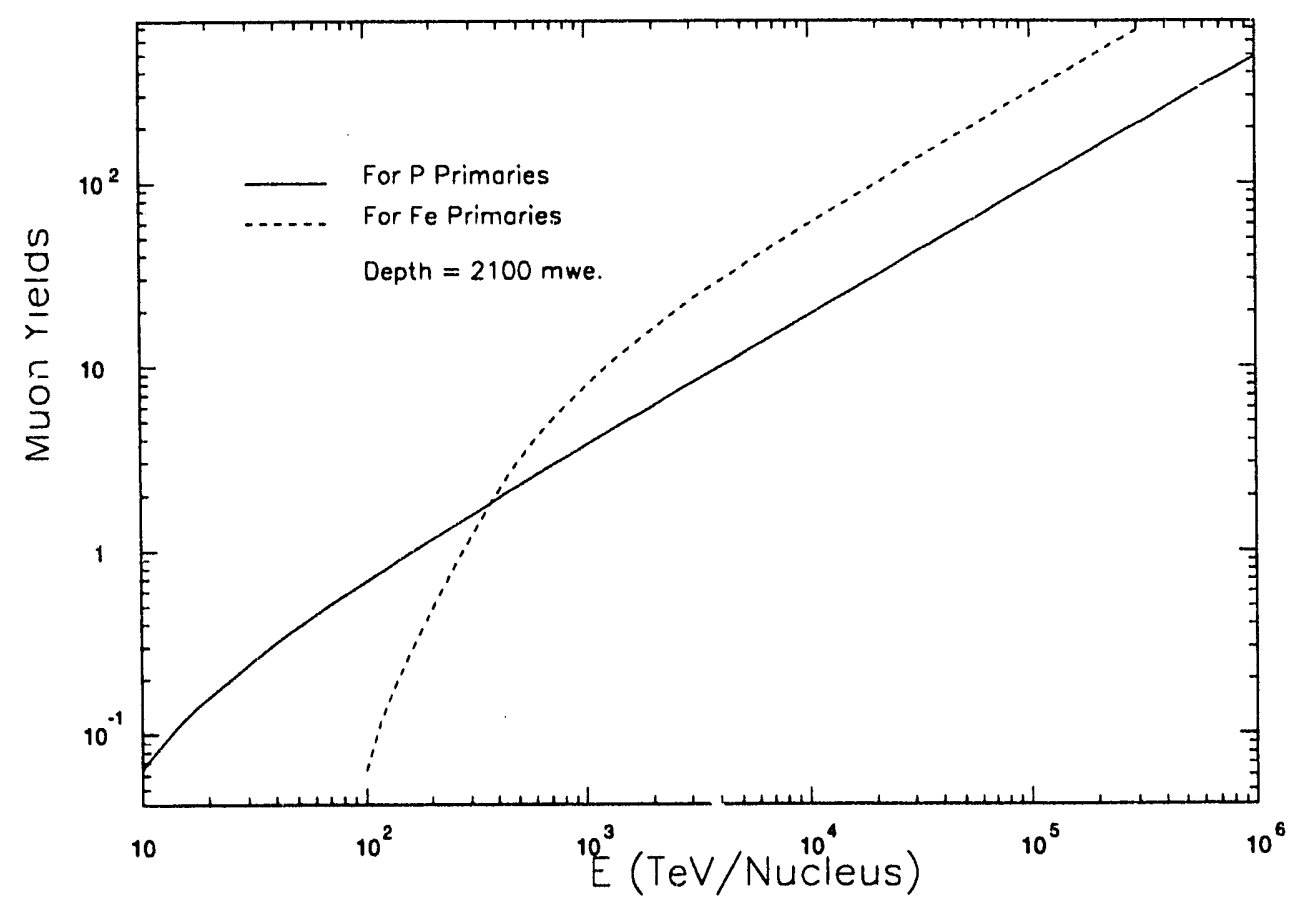

Figure 6.6: Muon yield at 2090 mwe, proton versus iron primaries.

muons produced by different primaries of different atomic mass number $A$ :

$$
N_{\mu}\left(E>E_{\mu}^{t h}\right)=\frac{K A \sec \theta}{E_{\mu}^{t h}} \cdot\left(\frac{E_{\mu}^{t h}}{E / A}\right)^{\alpha} \cdot\left(1-\frac{E_{\mu}^{t h}}{E / A}\right)^{\beta}
$$

Here $E_{\mu}^{\text {th }}$ is the muon threshold :nergy, $E$ is the primary energy, and $\theta$ is the zenith angle; $\alpha, \beta$, and $K$ are constents. For a particular primary energy, e.g. $E=1000$ $\mathrm{TeV}, N_{\mu}$ is 4 for proton primaries and 8 for Fe primaries at the particular zenith angle of $10^{\circ}$ and $E_{\mu}^{\text {th }}=0.83 \mathrm{TeV}$. If the threshold energy is reduced, more muons will reach the detector. For example, with $E=10^{3} \mathrm{TeV}$ and $\theta=10^{\circ}$, but $E_{\mu}^{\text {th }}$ $=0.70 \mathrm{TeV}$, the number of muons at the detector level are 5 and 11 for proton and iron primaries respectively. This illustrates how the rock overburden affects the multiplicity and relationship between $N_{\mu}$ and $A$. 
(iii) The energy of the produced hadrons (pions and kaons) from cosmic ray primaries of different atomic number ("light" or "heavy").

Pions produced from Fe-air collisions have, on average, a lower energy than do pions produced by proton showers of the same primary energy. The energy per nucleon within a primary nucleus determines the pion yield and therefore the muon yield. If we consider the muon threshold energy at a particular value, Fe primaries require higher energy to produce muons which reach the underground detector than do proton primaries. On the other hand, if there is enough incident energy such that any incident primary produces muons which penetrate the rock overburden, then Fe primaries will give more muons than proton primaries for the following reason: For iron versus proton cosmic ray primaries of the same energy, Fe nuclei have much larger cross sections with air nuclei, consequently they interact with air nuclei as soon as they enter the atmosphere. Then the flight paths of pions from an Fe-induced hadronic shower are through a more tenuous medium, allowing the pions more opportunity to decay into muons than is available to proton-produced pions which are created at interaction points deeper in the atmosphere. Therefore the muon multiplicity may be relatively higher for $\mathrm{Fe}$ initiated showers.

The general relationships between muon multiplicity, primary energy and primary composition can be seen with the model simulations. Primary energy distributions for different bundle multiplicities and for different primary composition are shown in Figs. 6.7 (a), (b), and (c). The distributions are based upon the CMC model and the multiplicities are for muons detected. Fig. 6.7(a) shows the various distributions from a mixture of all nuclei according to the spectrum in the CMC model. Fig. 6.7(b) and Fig. 6.7(c) show distributions for $p$ and Fe primaries using the same model. The dark shaded areas in the figures depict events with multiplicity $N_{\mu} \geq 8$. Comparing the yield for high multiplicity events from proton primaries versus $\mathrm{Fe}$ primaries, one can infer that the heavier nuclei are mostly responsible for 

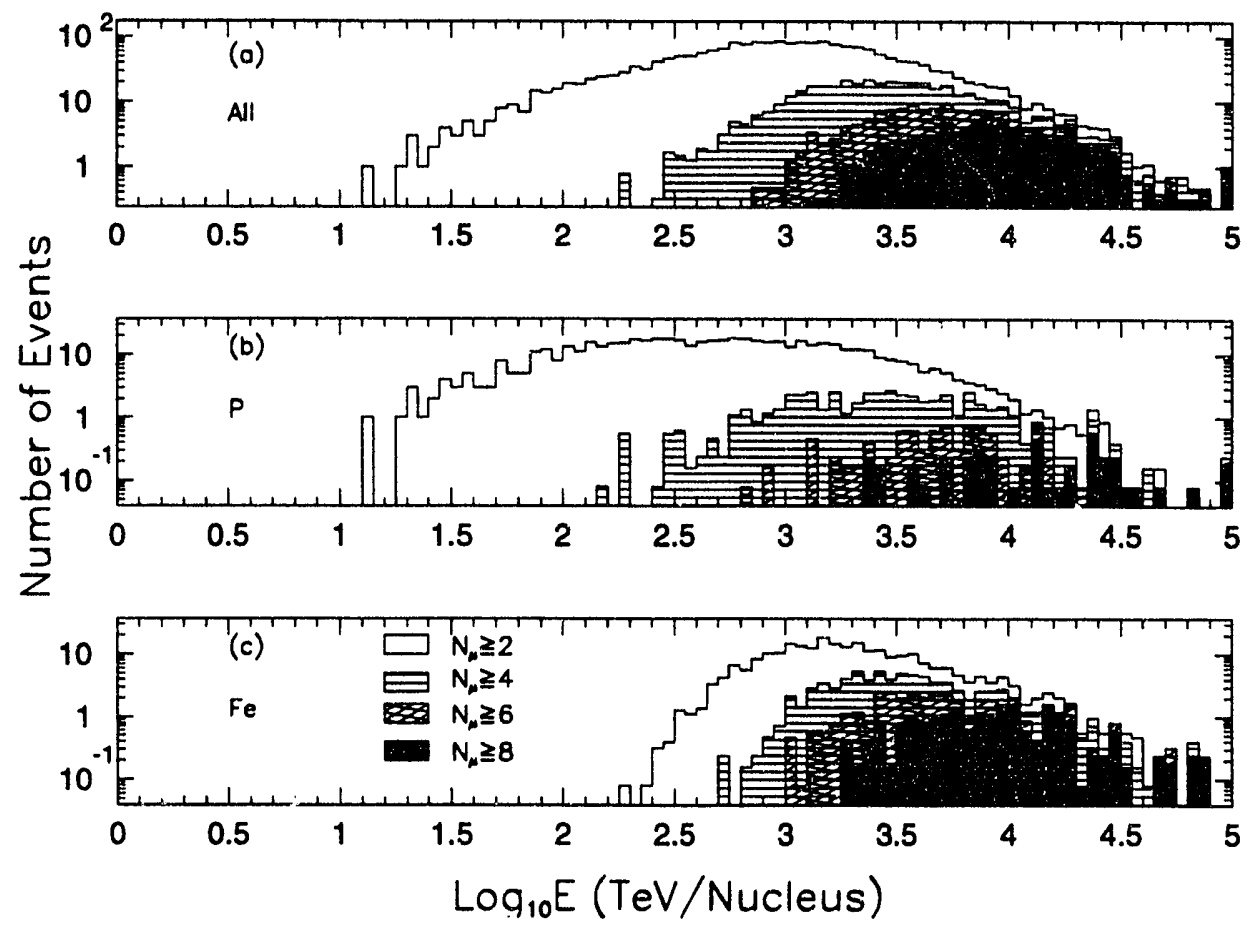

Figure 6.7: Relative rates for bundle multiplicity as a function of primary energy: (a) Mixture of nuclei (CMC model); (b) primary protons; (c) primary iron nuclei.

these events. For an Fe primary, the minimum energy should be at least $10^{3} \mathrm{TeV}$. Very high energy protons can also yield high multiplicity events, but at a much reduced rate. Unfortunately, for the data of this Thesis, energy tagging of muon bundles using the surface array had not commenced, and so the higher inultiplicity events can be either from heavier elements or from the high energy protons.

\subsection{Absolute Rate for Multiple Muon Events}

The differential flux of nuclei of mass $A$ at energy $E$ with normalization constants $K_{i}$ and power factor $\gamma_{i}$ is given by

$$
\frac{d N}{d E}=\sum_{i} K_{i}(A)\left(\frac{E}{1 G e V}\right)^{\gamma_{i}(A)}
$$


where $\frac{d N}{d E}$ and $K_{i}$ are in units of nuclei $/\left(\mathrm{m}^{2} \mathrm{~s}\right.$ sr $\mathrm{GeV} /$ nucleus). The parameters $K_{i}$ and $\gamma_{i}$ are model dependent; the index $i$ denotes the different primaries. The primaries considered here are $\mathrm{H}, \mathrm{He}, \mathrm{CNO}, \mathrm{Si} / \mathrm{Mg}$ and $\mathrm{Fe}$. The flux integrated over the energy range between $E_{\min }$ and $E_{\max }$ for all type of primaries is given by

$$
N=\sum_{i} \frac{K_{i}(A)}{\gamma}\left[E_{\max }^{\gamma_{i}(A)}-E_{\min }^{\gamma_{i}(A)}\right]
$$

where $N$ is in nuclei $/ \mathrm{m}^{2}$ s sr.

The muon bundle rate is given by [47]

$$
\Phi_{n}\left(\mathrm{~s}^{-1}\right)=\sum_{i} K_{i}(A) \int E^{\gamma_{i}(A)} P_{n}(E, A) d E
$$

where $P_{n}(E, A)$ is the probabiiity to sample $n$ muons at the detector. The probability $P_{n}(E, A)$ depends on the following: (i) The shape of the earth above the detector; (ii) the rock properties (density, average $Z$ and $A$ ); (iii) the detector geometry and efficiency; and (iv) the track reconstruction efficiency.

Calculation of $P_{n}(E, A)$ involves th= muon threshold energy $E_{\mu}^{\text {th }}$, the average number of muons $\left\langle N_{\mu}\right\rangle$, the lateral distribution of muons from the core and the detection of $n$ muons out of the $N_{\mu}$ muons. An analytical formula for calculating the muon bundle rate for specific zenith and azimuthal angle ranges is given by [48]

$$
\Phi_{n}\left(\mathrm{~s}^{-1}\right)=\sum_{i} K_{i}(A) \int_{E_{\min }}^{E_{\operatorname{mes}}} E^{\gamma_{i}(A)}\left[\int_{0}^{\infty} \cos \theta \mathcal{R} d \mathcal{R} \int_{0}^{2 \pi} d \phi \frac{d P_{n}(E, A)}{d \sigma}\right] d E
$$

where

$$
\frac{d P_{n}(E, A)}{d \sigma}=\sum_{N_{\mu} \geq n} P_{\left\langle N_{\mu}\right)}\left(N_{\mu}\right) \int_{0}^{2 \pi} d \varphi \int_{0}^{\theta_{\operatorname{mas}}} \frac{d p_{n}}{d \omega d \sigma} \sin \theta d \theta .
$$

The $P_{\left\langle N_{\mu}\right\rangle}\left(N_{\mu}\right)$ is the probability to get $N_{\mu}$ muons for $\left\langle N_{\mu}\right\rangle\left(E, A_{;}>E_{\mu}^{t h}\right)$ and $\frac{d p_{n}}{d w d \sigma}$ is the probability to detect $n$ muons out of $N_{\mu}$ muons in the detector. $d \sigma=\cos \theta \mathcal{R} d \mathcal{R}$ is the area element where the bundle core impacts on a plane normal to the direction given by $\omega(\theta, \varphi)$. The allowe' maximum zenith angle is $\theta_{\max }$.

A calculation of the above type has been already included in the Monte Carlo simulation program. The formula for the muon bundle rate can be expressed in 
simplified form by the following equation:

$$
\Phi_{n}\left(\mathrm{~s}^{-1}\right)=N \cdot \mathcal{A} \cdot \Omega \cdot \frac{N_{\text {det }}}{N_{\text {gen }}} \cdot \eta .
$$

Here $N$ is the total flux, integrated over the given energy range and summed over all primary particles; $\mathcal{A}$ is the total area considered for event generation (a circle of 30 meter radius taken to be perpendicular to the muon bundle direction), and $\Omega$ is the solid angle. The correction factor $\eta$ is introduced to compensate for approximations inherent in 'he above equation, and to accommodate the uncertainties in the rock properties and the geometry of the rock overburden. All events $\left(N_{\text {gen }}\right)$ were generated over the zenith angle range between $\theta=0$ and $\theta=\theta_{\max }$. The solid angle within the above range is given by

$$
\Omega=2 \pi\left(1-\cos \theta_{\max }\right) \text {. }
$$

If the number of events with $n$ muons observed by the central detector and shield is $N_{o b}$ within the total live time $T$ seconds, then the observed rate is given by $N_{o b s} / T$. The total Soudan 2 live time for our data set is $T=5.38 \times 10^{6} \mathrm{~s}$, and $\Omega=\pi$ steradians for the zenith angle range from 0 to $60^{\circ}$. The absolute event rates for different multiplicities are shown in Fig. 6.8 for the data and for all four models. We find that good agreement between the data and the models is obtained provided that the models are corrected by the (arbitrary) factor $\eta=1.4$. In Fig. 6.8, solid circles depict the data, while the open symbols show the models. Our procedures here provide a comparison of absolute multiplicity rates with the models which is very crude. Nevertheless, we believe the comparison to be informative, since $\eta$ provides a measure of overburden uncertainties.

We have explored the effect of overburden uncertainties on the event rates by changing the rock density. The density values $2.55 \mathrm{~g} \mathrm{~cm}^{-3}, 2.65 \mathrm{~g} \mathrm{~cm}^{-3}$ and 2.80 $\mathrm{g} \mathrm{cm}^{-3}$ have been tried instead of $2.93 \mathrm{~g} \mathrm{~cm}^{-3}$ which was used above. The CMC model is chosen for Monte Carlo event generation with 'he new trial densities for the rock. The effect of altering the rock properties is clearly seen in Fig. 6.9(a), 
where the solid circles depict the data, open symbols depict the model predictions with the densities given above (all with $\eta=1.0$ ).

In the calculation of muon threshold energy, one must consider not only the rock density but also the parameters $a$ and $b$ in Eq. (5.3). These parameters, which characterize ionization energy loss and radiation energy loss for a muon on its path through the rock, depend on the average $Z$ and $A$. The values $a=2.5 \mathrm{MeV} \mathrm{g}^{-1} \mathrm{~cm}^{2}$ and $b=4.0 \times 10^{-6} \mathrm{~g}^{-1} \mathrm{~cm}^{2}$ are taken from Soudan 1 experiment [42]. Better measurements of $Z$ and $A$ are required in order to make more meaningful comparisons of model event rates and the data. According to the Minnesota Geological Survey, the Soudan 2 detector is surrounded mostly by greenstone [43]. For greenstone $\langle Z / A\rangle$ $=0.496,\left\langle Z^{2} \mid A\right\rangle=6.01$ and the average density is $2.80 \mathrm{~g} \mathrm{~cm}^{-3}$ (upper limit). Using these values as input, the $a$ and $b$ in Eq. (5.3) have been calculated for $10 \mathrm{TeV}$ muons. The Bethe-Bloch equation is used to calculate a (for ionization loss) [44]; the equations given in reference [49] for the energy losses due to bremsstrahlung, pair production and nuclear interaction are used to calculate $b$. For greenstone rock, the values $a=2.23 \times 10^{-6} \mathrm{TeV} / \mathrm{g} \mathrm{cm}^{-2}$ and $b=4.27 \times 10^{-6} / \mathrm{g} \mathrm{cm}^{-2}$ are obtained for $10 \mathrm{TeV}$ muons. The above values for $a$ and $b$ (hereafter refered to as $a_{2}, b_{2}$ ) and the average rock density $2.80 \mathrm{~g} \mathrm{~cm}^{-3}$ have been used to calculate threshold muon energy which is the input for Monte Carlo program to simulate events for all four models.

Fig. 6.10 shows the multiple muon event rates calculated for the rock density $2.80 \mathrm{~g} \mathrm{~cm}^{-3}$ and with $a_{2}$ and $b_{2}$. Here, comparison of the model predictions to the data is carried out without the introduction of a correction factor $(\eta=1.0)$. 


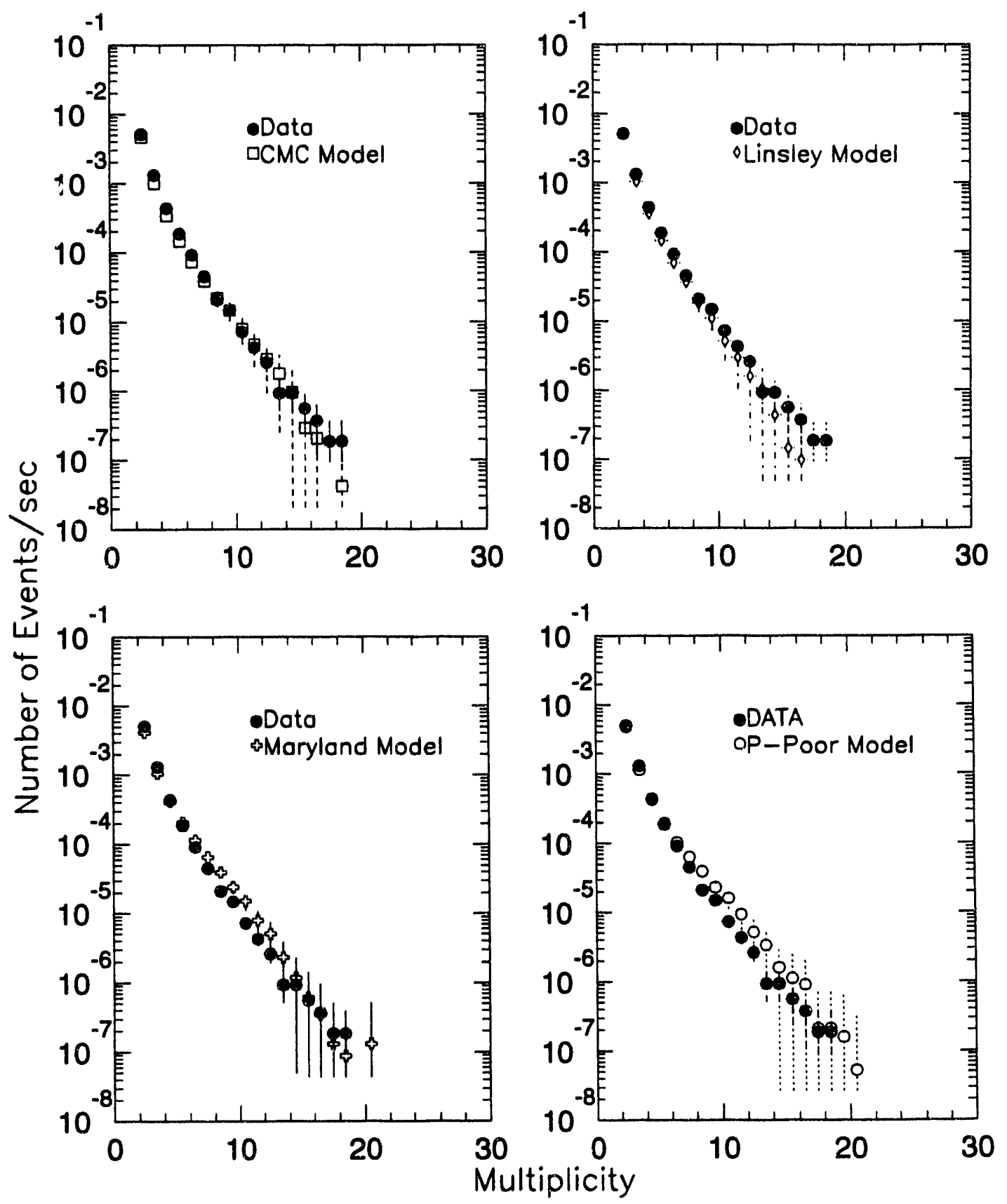

Figure 6.8: Comparison of muon bundle rates as a function of multiplicity, for the four composition models using a rock density of $2.93 \mathrm{~g} \mathrm{~cm}^{-3}$ and using the $a$ and $b$ from Soudan 1. Absolute rates for the models have to be multiplied by the factor $\eta$ $=1.4$ to compare with the data. 


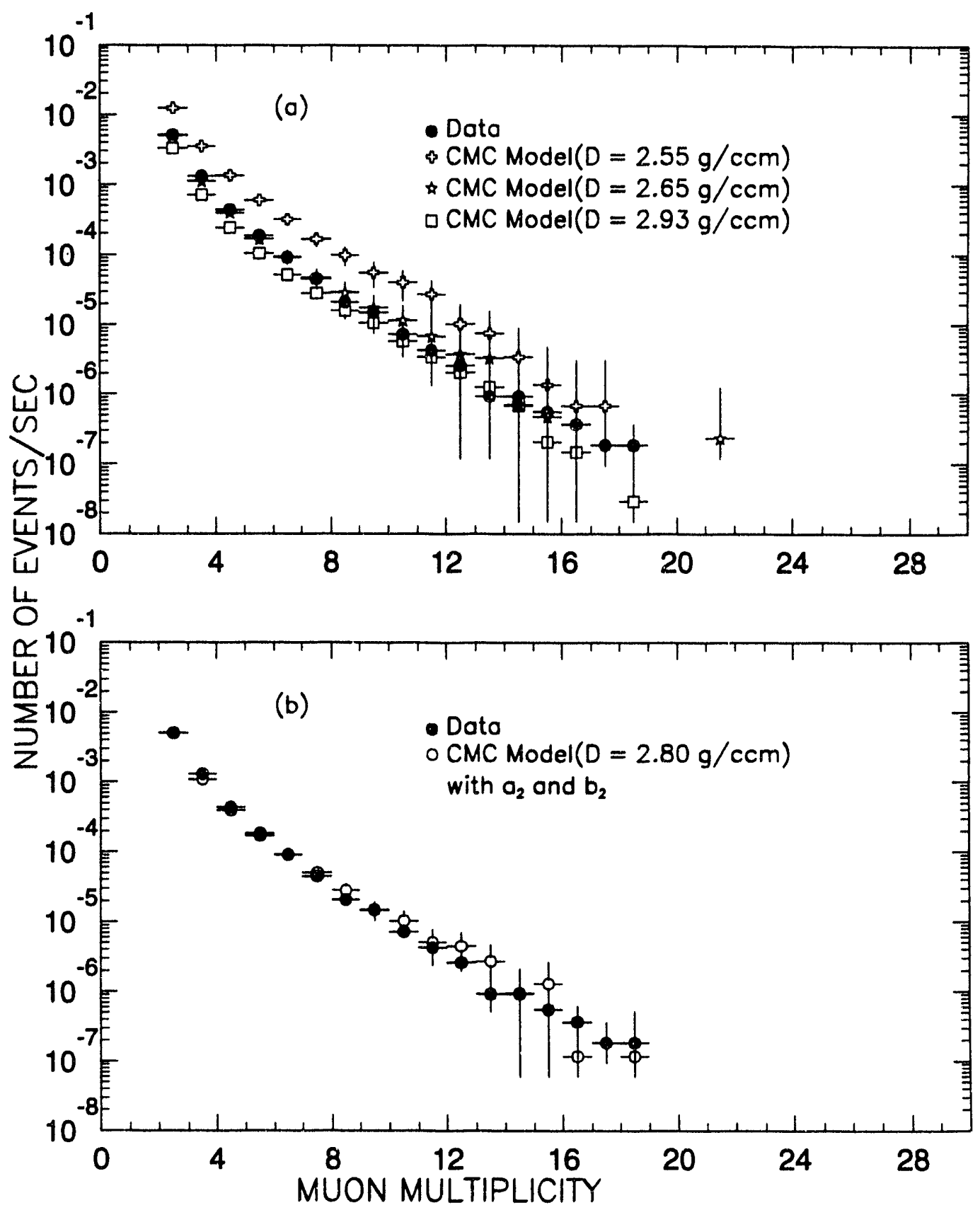

Figure 6.9: Multiple muon bundle rates using the CMC model (a) for three different rock densities and using $a$ and $b$ and (b) for the density $2.80 \mathrm{~g} \mathrm{~cm}^{-3}$ and using $a_{2}$ and $b_{2}$. 


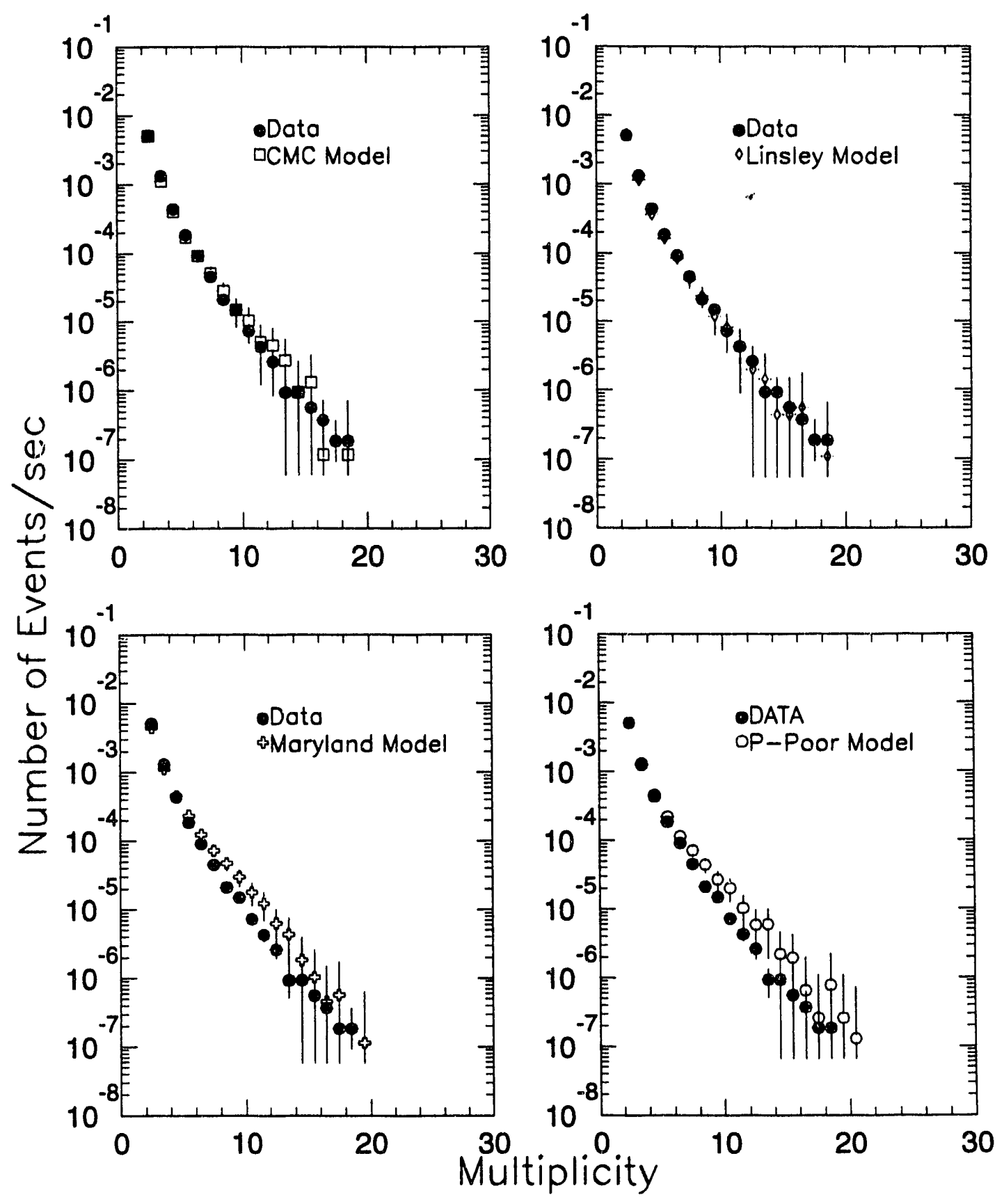

Figure 6.10: Comparison of muon bundle rates as a function of multiplicity, for the four composition models. Events for models were generated using the rock density $2.80 \mathrm{~g} \mathrm{~cm}^{-3}$ and using $a_{2}$ and $b_{2}$. 


\subsection{Muon Separation within Bundles}

With underground muon bundles, the measurement of muon separation $r$ from the shower core is difficult because of uncertainty in the location of the shower core. For this reason we have analyzed our data using the distribution of perpendicular distances $R_{i j}$ between the $i^{\text {th }}$ and $j^{\text {th }}$ muon for all $i \neq j$ within an underground muon bundle. Of course, higher multiplicity events give more pairs than lower multiplicity events; specifically, a bundle of $N$ muons gives $N(N-1) / 2$ pairs. To give equal weight to each event for events with all multiplicities, a weighting factor $\left[\frac{N(N-1)}{2}\right]^{-1}$ is applied to each pair, where $N \geq 2$.

The intermuon separation distribution $\frac{d N}{d R_{i j}}$ is shown in Fig. 6.11 for the data and for the four composition models. Spatial separations between muons within individual bundles of up to 30 meters have been observed. The models yield distributions with shapes similar to but narrower than the data. All four of the composition models yield similar distributions for intermuon separations in Soudan 2; at 2090 mwe it appears that the distribution in the intermuon separation $R_{i j}$ is nearly independent of primary cosmic ray composition. Separations of up to 20 meters are shown in Fig. 6.12 for the four models. The average separation changes only by $6 \%$ between the extreme models which are the Linsley model (light) and the Maryland model (heavy). The average $R_{i j}$ for the Linsley model and the Maryland model are 6.9 and 7.2 meters respectively, to be compared with $7.1 \pm 0.3$ meters for the data sample. Similar results have been reported by the MACRO group [52] at a depth of 3800 mwe, which is distinctly deeper than the Soudan depth. 


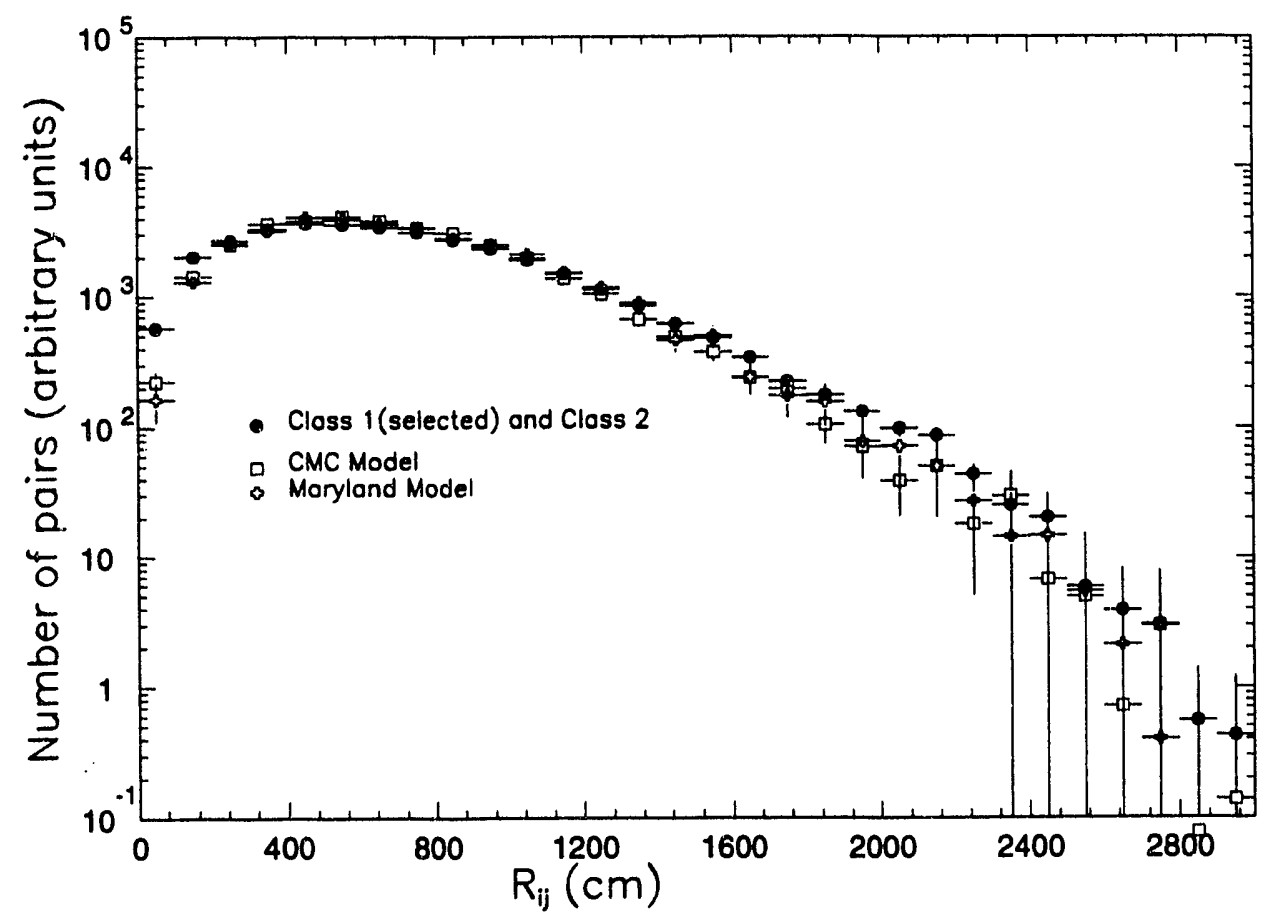

Figure 6.11: Distribution of separations between pairs of muons, in the data and in the models.

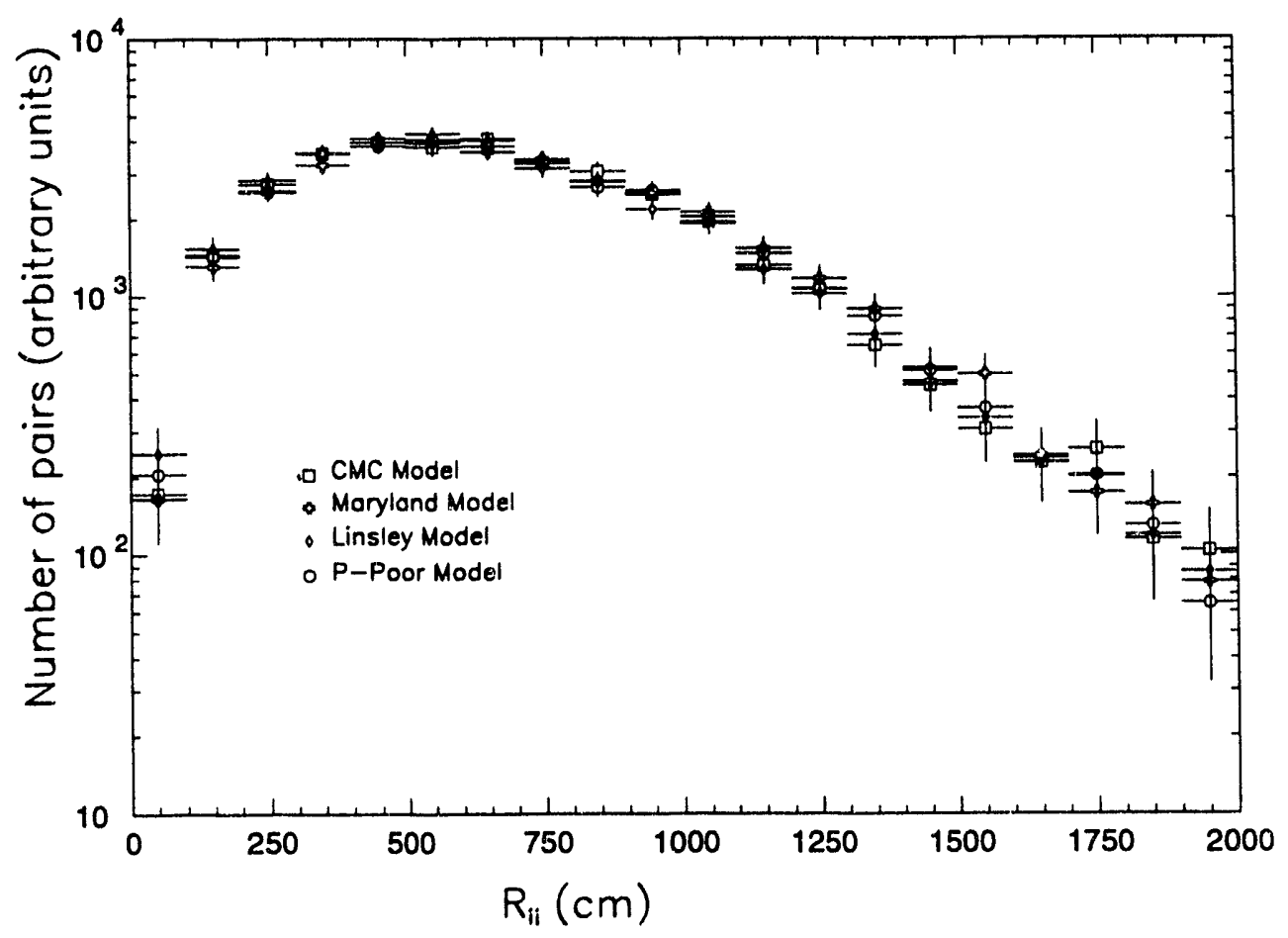

Figure 6.12: Distribution of separations between pairs of muons, for the models only. 


\section{Chapter 7 \\ Search for Correlations in Properties of Muon Bundles}

Results from searches for correlations involving bundle multiplicity, intermuon separation, and zenith angle are presented below. The extent to which these observations are accounted for in each of the four models for nuclear composition of cosmic rays, is discussed. The average rock density $2.80 \mathrm{~g} \mathrm{~cm}^{-3}$ and the calculated values for $a_{2}$ and $b_{2}$ (see Section 6.4) have been used for Monte Carlo simulation.

\subsection{Average Multiplicity versus Zenith Angle}

The average muon multiplicity has been calculated for different zenith angle bins in order to search for correlations between multiplicity and zenith angle. More specifically, the cosine of the zenith angle is binned over the interval from $15^{\circ}$ to $60^{\circ}$; for large zenith angles $\left(40^{\circ}\right.$ to $\left.60^{\circ}\right)$ the bin size is doubled (from 0.02 to 0.04 ) in order to more nearly equalize the bin to bin statistics. Within each zenith angle bin, the multiplicity distribution is obtained and $\left\langle N_{\mu}\right\rangle$ is calculated from the distribution. The statistical errors which are not shown in Fig. 7.1 are smaller than the symbols. The data show no variation in the average multiplicity with zenith angle for $15^{\circ} \leq$ $\theta \leq 60^{\circ}$, as can be seen in Fig. 7.1. As shown in this figure, the same lack of variation is exhibited by all four of the composition models, namely the CMC model (Fig. 7.1(a)), the Linsley model (7.1(b)), the Maryland model (7.1(c)) and the P-Poor model (7.1(d)). 


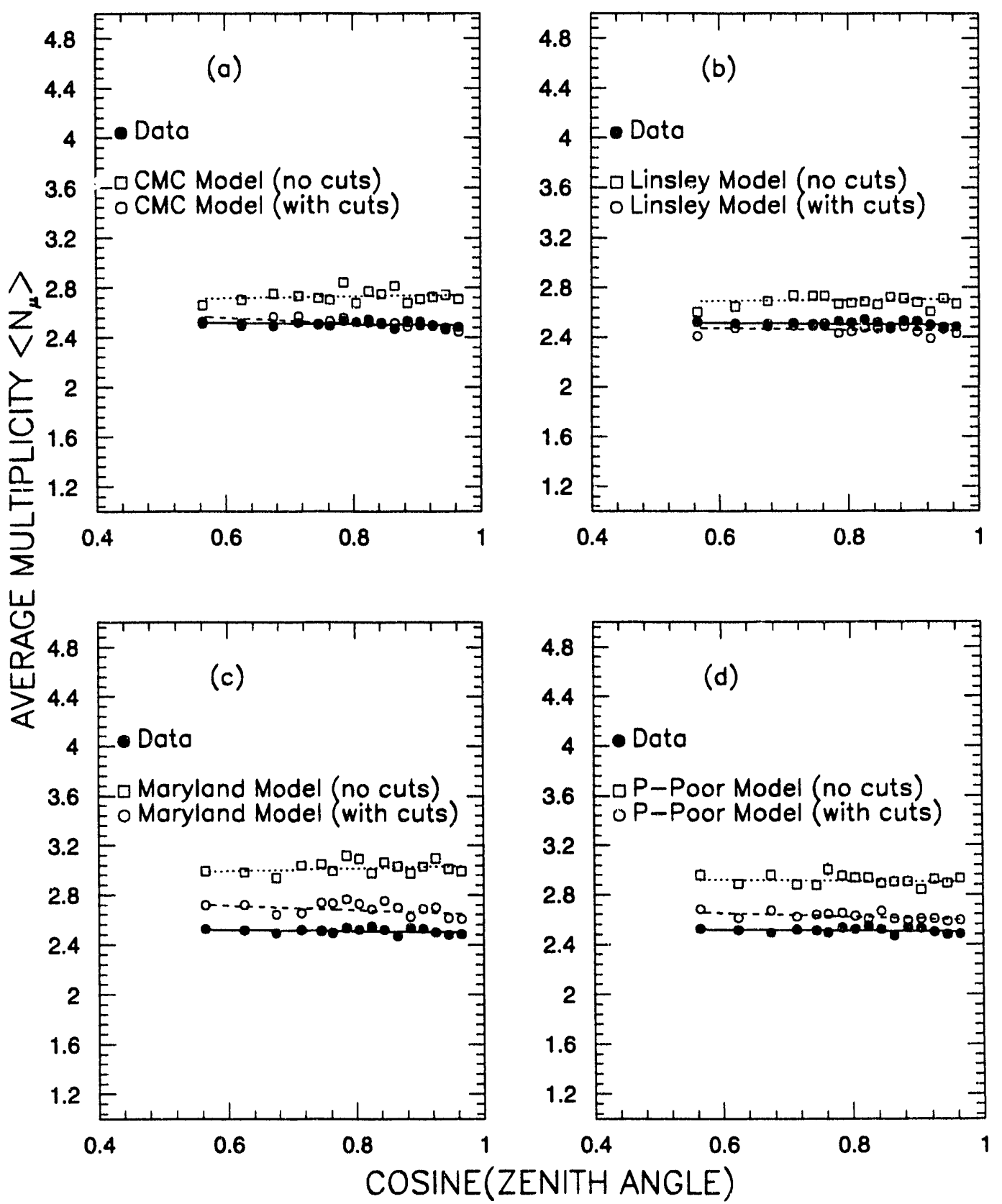

Figure 7.1: Average muon multiplicity versus zenith angle. The data is compared to the four models of primary cosmic ray composition. 
Table 7.1: Fitting Results

\begin{tabular}{|l|c|c|c|c|}
\hline \multicolumn{2}{|c|}{} & Slope & Intercept & $\chi^{2} / \mathrm{DF}$ \\
\hline \multicolumn{2}{|c|}{ Data } & 0.045 & 2.54 & 1.03 \\
\hline \multirow{2}{*}{$\begin{array}{l}\text { Model } \\
\text { Linsley }\end{array}$} & With cuts & -0.253 & 2.71 & 3.07 \\
\cline { 2 - 5 } Model & Without cuts & 0.079 & 2.67 & 2.67 \\
\cline { 2 - 5 } Maryland & With cuts & -0.057 & 2.51 & 2.91 \\
\cline { 2 - 5 } Model & Without cuts & 0.032 & 2.67 & 4.34 \\
\cline { 2 - 5 } P-Poor & Without cuts & -0.176 & 2.82 & 3.74 \\
\cline { 2 - 5 } Model & With cuts & -0.150 & 2.74 & 0.79 \\
\cline { 2 - 5 } & Without cuts & -0.049 & 2.95 & 1.17 \\
\hline
\end{tabular}

In the four figures, the solid circles represent the data; the solid line connecting the data points shows the best straight line fit with chi-square per degree of freedom $\left(\chi^{2} / D F\right)=1.03$. The slope and intercept of straight line fits to the data and to the model predictions are given in Table 7.1. The predictions for the models (open circles in the figures) are obtained by calculating $\left\langle N_{\mu}\right\rangle$ using muon tracks from the Monte Carlo simulations. The event selections and cuts as described in Chapters 3 and 6 , have been applied to both the data and to the simulated events. Also shown in the figures (open squares) are predictions from the models obtained using a perfectly efficient detector. For the data and for all models, $\left\langle N_{\mu}\right\rangle$ seen to be independent of the zenith angle. In Chapter 6 we reported that the heavy composition models predict higher average muon multiplicities than observed in the data or predicted in the lighter composition models. In Fig. Fig. 7.1 we observe that this is also the case in every zenith angle bin. 


\subsection{Intermuon Separation versus Zenith Angle}

We have calculated the average intermuon separation within bundles $\left\langle R_{i j}\right\rangle$ for various zenith angle bins as follows: all multiple muon events with multiplicity two and above in the data having cosine of the zenith angle within the range between 0.97 and 0.50 , have been selected. The binning in zenith angle is set up as described in the previous section. The perpendicular distance between each muon pair within an event is then calculated and the distribution of $R_{i j}$ values is obtained. The average intermuon separation $\left\langle R_{i j}\right\rangle$ is then calculated for each zenith angle bin. Fig. 7.2 shows the correlation between intermuon separation and zenith angle for bundle multiplicities two and three. It can be seen that there is a mild increase in $\left\langle R_{i j}\right\rangle$ with increasing zenith angle. The error for each data point (solid circles) predominantly reflects the shield's limited spatial resolution. Uncertainties in track locations can be as large as $30 \mathrm{~cm}$ to $50 \mathrm{~cm}$. The solid line shows the straight line fit to the data points. In the Fig. $7.2(a)$ the binned $\left\langle R_{i j}\right\rangle$ points are shown with the points for the CMC model. The open circles show simulated data with cuts while the open squares show simulated data for a perfect detector. The Linsley model is displayed similarly in Fig. 7.2(b). Fig. 7.2(c) shows the Maryland model; Fig. 7.2(d) shows the P-poor model.

Fig. 7.3 shows the correlation between $\left\langle R_{i j}\right\rangle$ and the cosine of zenith angle with bundle multiplicity of four and higher; results from the four models are also shown. The same trend is observed for the data and for all four model simulations. However, as noted previously, the data consistently exhibits an $\left\langle R_{i j}\right\rangle$ which is slightly higher than the model predictions. The average $R_{i j}$ at Soudan is observed to increase slightly with increasing zenith angle. Similar trends were reported by the Fréjus experiment at 4850 mwe [14] and MACRO at Gran Sasso at 3780 mwe [52]. 

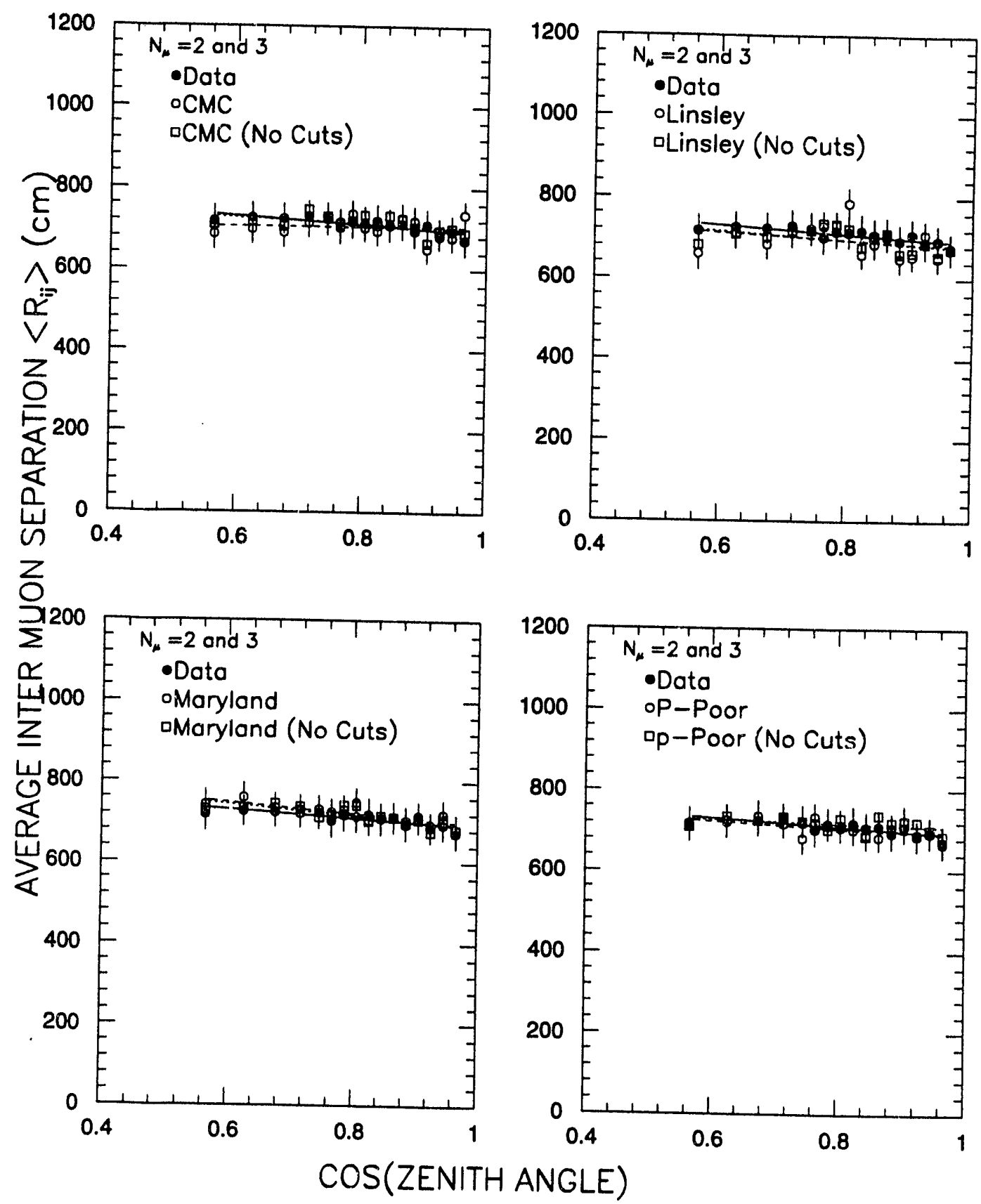

Figure 7.2: Intermuon separation versus zenith angle for bundle multiplicities 2 and 3 , compared to the predictions of the composition models. 

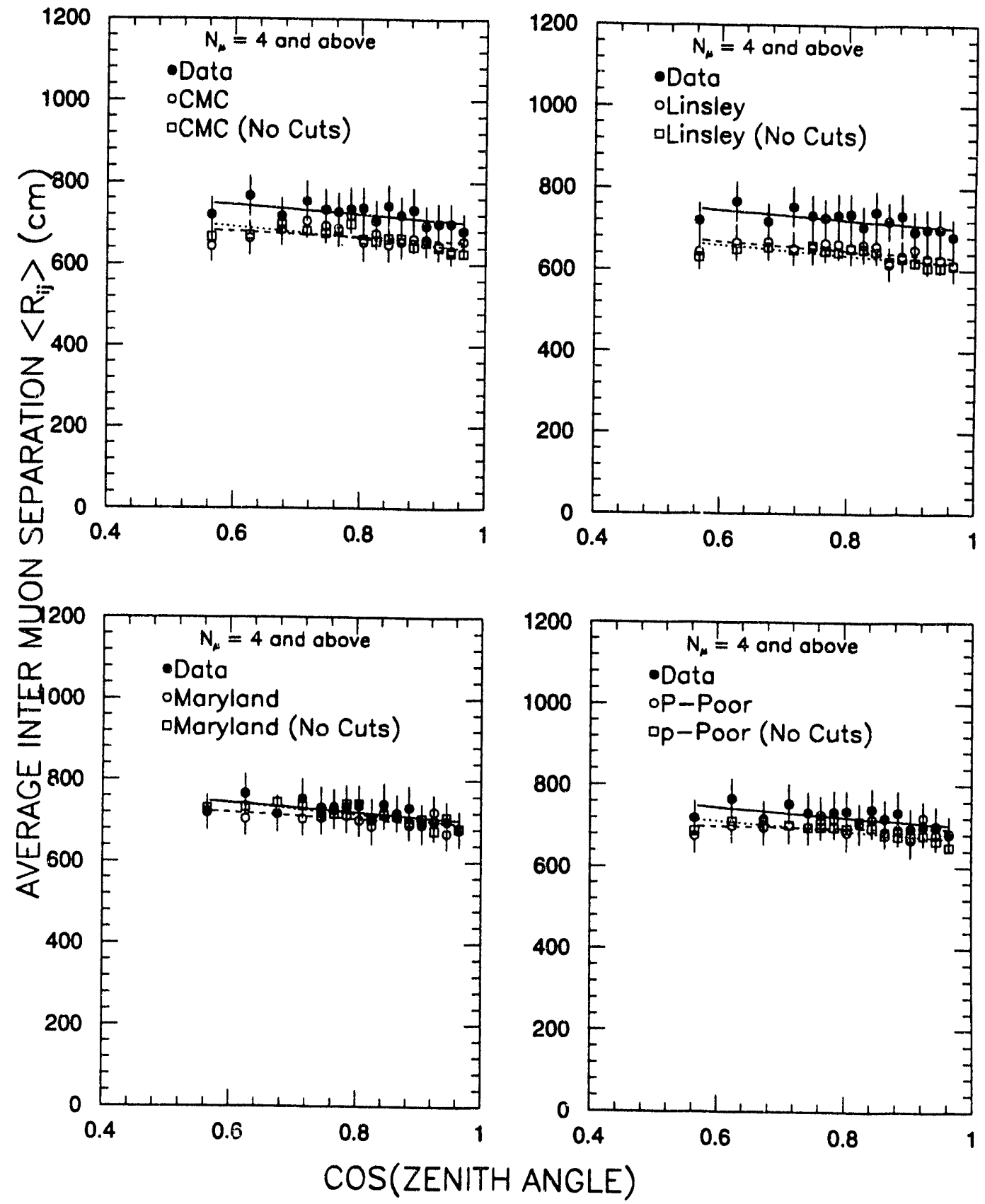

Figure 7.3: Intermuon separation versus zenith angle, for multiplicities $N_{\mu} \geq 4$, compared to the composition models. 

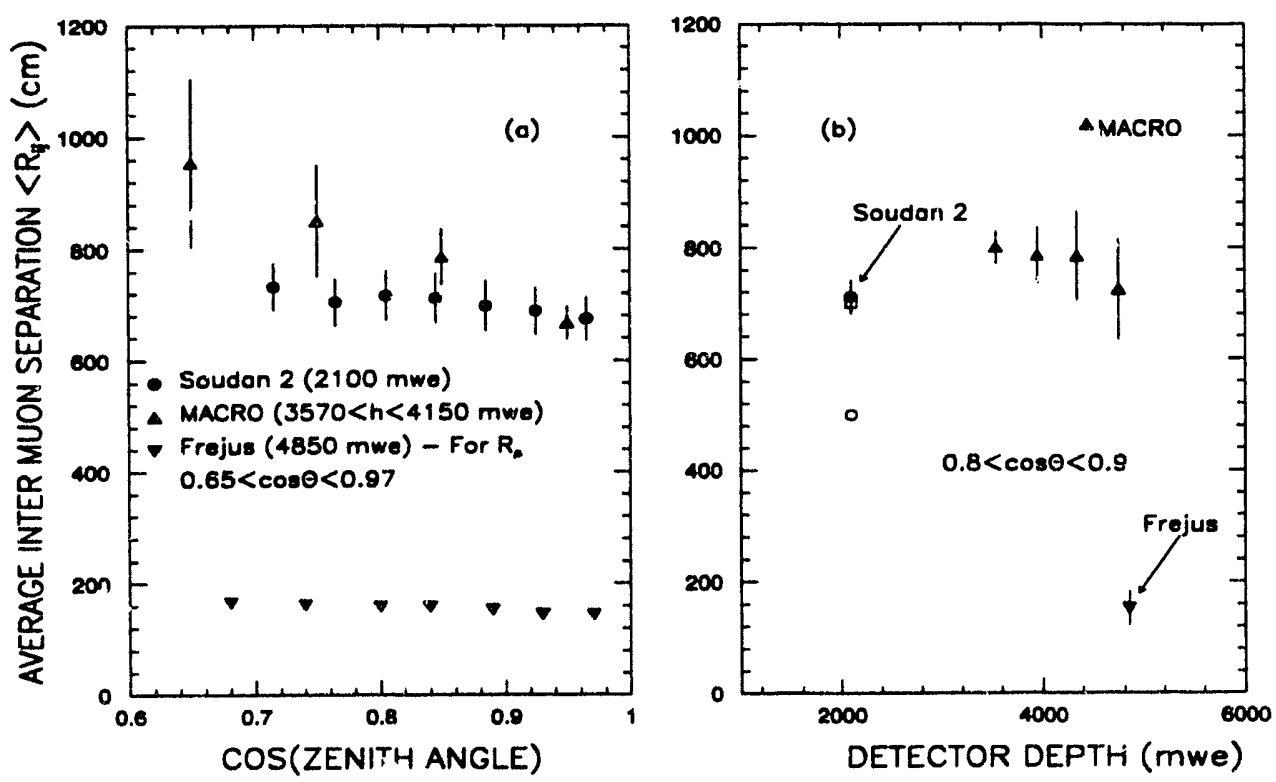

Figure 7.4: (a) The average intermuon separation versus zenith angle for three different experiments; (b) the average intermuon separation versus depth.

It may be possible to explain qualitatively a mild rise of $\left\langle R_{i j}\right\rangle$ with increasing zenith angle as follows: The rock overburden is of cours. greater for larger zenith angles. With larger zenith angle, the energy of primary cosmic rays which can produce muons at Soudan 2 will, on average, necessarily be higher. With higher energy in the primary cosmic ray interaction, secondary particles such as pions, and consequently their daughter muons, will tend to be produced into more forward dir zctions (in the Laboratory System). On the other hand, for such large zenith angle events there are longer flight paths for the rumons through the Earth to reach the underground detector; this provides more time in-flight for muons within a bundle to separate (transversely). The latter trend has to dominate over the collimation tendency at production, in order for $\left\langle R_{i j}\right\rangle$ to increase with increasing zenith angle.

Fig. $7.4(\mathrm{a})$ shows the correlation between $\left\langle R_{i j}\right\rangle$ and $\cos \theta$ for three experiments: 
Soudan 2 (solid circles), MACRO (upward triangles) [3] and Fréjus (downward triangles) [14]. In the case of Fréjus, the distance given in the plot is the average distance $r_{0}$ between muons and the estimated shower core, rather than the actual muon separation. As mentioned earlier, all three experiments show the mean separation to increase with increasing zenith angle. For Soudan 2 , the $\left\langle R_{i j}\right\rangle$ points represent detector dependent values while the MACRO points are detector independent values. Clearly a detector independent value for the Soudan 2 is desirable in order that the trend suggested by the MACRO data points can be checked. The open circle in Fig. 7.4(b) shows the Monte Carlo prediction for $\left\langle R_{i j}\right\rangle$ at Soudan 2 using all muons in the simulation at 2090 mwe. The open square in the same figure shows $\left\langle R_{i j}\right\rangle$ for the detected muons. The calculation of $\left\langle R_{i j}\right\rangle$ for this point includes all selections and cuts which are applied to the data. Fig. 7.4(b) shows a correlation between $\left\langle R_{i j}\right\rangle$ and rock depth as reported by MACRO [52]. According to the MACRO result, the mean muon separation decreases with increasing depth.

For completeness, we include results from other experiments. For the mean distance from the shower core, Kolar Gold Field [16] has reported a value of $r_{0}=$ $1.09 \pm 0.23 \mathrm{~cm}$, at a depth of $6045 \mathrm{mwe}$ and with an effective detector area of $36 \mathrm{~m}^{2}$. For the Utah experiment [53], $r_{0}$ is reported to be $10.1 \mathrm{~m}$ and $7.9 \mathrm{~m}$ at $4000 \mathrm{mwe}$ and 4800 mwe :espectively. They observed these values at a particular zenith angle of $62.5^{\circ}$. For vertical muons, Homestake [53] obtained $r_{0}=4.4 \mathrm{~m}$ at a depth of 4400 mwe.

\subsection{Intermuon Separation versus Multiplicity}

The parametrizations used in the Monte Carlo simulations do not incorporate any correlation between muon multiplicity and lateral distribution [37]. However, one might expect that such a correlation exists for the following reason: For primary cosmic ray interactions which occur at higher altitudes, the probability that 
they yield more muons underground is higher. This is because pions produced in cosmic ray - air interactions, where the medium is more tenuous, have an enhanced probability to decay before interacting with other atoms in the air. Moreover, from interactions very high in the atmosphere, one expects muons to arrive underground with increased lateral spread. The existence of correlations between bundle multiplicity, lateral distribution and interaction height has been inferred by C. Forti et al. [37] from simulations using $1000 \mathrm{TeV}$ primary protons at a particular zenith angle $\left(28^{\circ}\right)$ and slant depth (3000 mwe). No correlation was indicated by a similar simulation which considered iron primaries.

The relationship between intermuon separation and muon multiplicity for our data and for our model simulations is shown in Fig. 7.5. Both data and the reconstructed Monte Carlo events exhibit a linear dependence of average intermuon separation on bundle multiplicity. Results are shown for each individual multiplicity for $2 \leq N_{\mu} \leq 8$; events with higher multiplicities have been grouped in order to maintain comparable bin statistics. The data (solid circles) and the model predictions (open circles) are plotted together in Figs. 7.5(a), (b), (c), and (d). The open squares in the same figure are also for the models, calculated before cuts were applied.

The general features of the correlations between intermuon separation and muon bundle multiplicity which can be inferred from Fig. 7.5, are as follows:

1. The data indicate a correlation, with $\left\langle R_{i j}\right\rangle$ increasing with increasing multiplicity.

2. The points from the simulation do not coincide with the data points.

3. The simulation results, for an ideal detector, ie. without any cuts, exhibit an opposite trend, that is $\left\langle R_{i j}\right\rangle$ decreases with increasing $N_{\mu}$.

4. With all cuts a!jplied, the simulation results indeed show an increasing trend as in the data, however the increase $\left\langle R_{i j}\right\rangle$ is more pronounced in 
the data than in any of the model simulations.

The average intermuon separation $\left\langle R_{i j}\right\rangle$ is $7.0 \mathrm{~m}$ for the models (the average for all four models), compared to $7.1 \pm 0.3 \mathrm{~m}$ for the data. The dependence of average intermuon separation on rock depth suggests that the "effective" depth that we used for the simulations is more than the actual depth. This situation may originate with rock density that we used in our simulations. The rock density 2.80 $\mathrm{g} \mathrm{cm}^{-3}$ was used. The effect of rock density on the simulations has been discussed in Chapter 6, section 6.2.2, where we found that a higher density corresponding to more overburden results in a smaller value for $\left\langle R_{i j}\right\rangle$. 


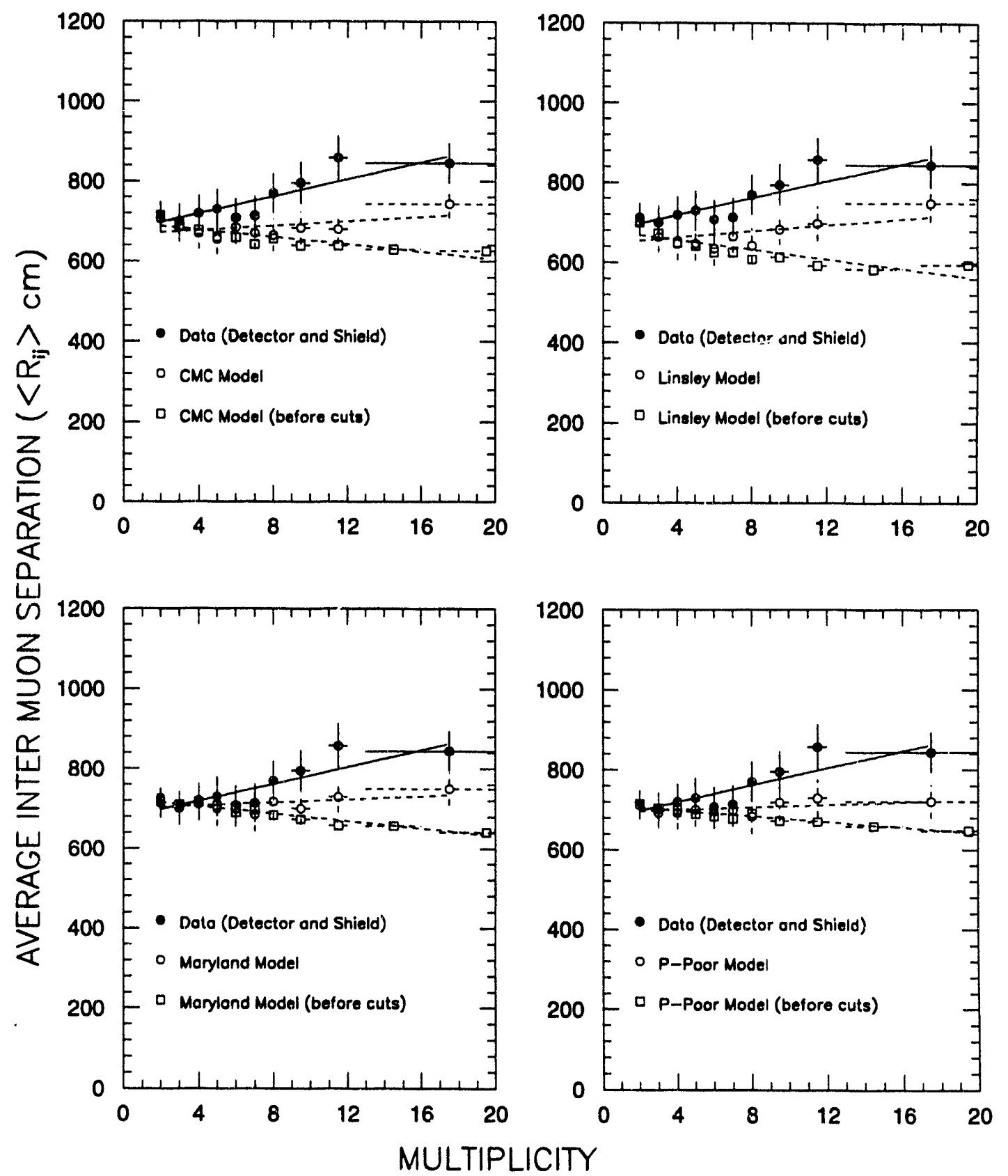

Figure 7.5: Intermuon separation versus bundle multiplicity; the data compared to predictions of the four composition models. 


\subsection{Protons versus Iron in Primary Cosmic Radiation}

In a simplified picture, one may consider primary cosmic rays to contain only protons and iron nuclei. The data can be fitt using the flux parameters for these nuclei taken from any of the composition models. To examine such a simplified picture, events for proton primaries and for Fe primaries have been extracted from the CMC simulation. For our current best values for the overburcien density and for the coefficients $a_{2}$ and $b_{2}\left(\rho=2.80 \mathrm{~g} \mathrm{~cm}^{-3} ; a_{2}=2.23 \times 10^{-6} \mathrm{TeV} \mathrm{g}^{-1} \mathrm{~cm}^{2} ; b_{2}\right.$ $\left.=4.27 \times 10^{-6} \mathrm{~g}^{-1} \mathrm{~cm}^{2}\right)$, the Monte Carlo simulations suffer from limited statistics. The modest simulation statistics is the origin of the relatively large errors for the multiplicity fit, and of the fluctuations of the Fe samples in Figures 7.7(a) and 7.7(b). Fig. 7.6 shows the multiplicity distribution for these selected events together with the muon multiplicity for the data sample. In order to compare the shapes of the distributions, the simulated distributions were normalized to the data for the twomuon events. To obtain the relative fractions of primary protons and Fe nuclei, we fit to observables in the data, a linear combination of proton and iron distributions in the observables. For any variable $\vartheta$,

$$
\vartheta_{c a l}=f_{p} \times \vartheta_{p}+f_{F e} \times \vartheta_{F e}
$$

where $f_{p}\left(f_{F_{e}}=1-f_{p}\right)$ is the fraction of protons (iron nuclei) in the primary flux. We calculate $\chi^{2}=\left(\vartheta_{c b s}-\vartheta_{c a l}\right)^{2} /\left(\delta \vartheta_{c b s}^{2}+\delta \vartheta_{c a l}^{2}\right)$ and use the program MINUIT to find $f_{p}$ and $f_{F e}$ corresponding to minimum $\chi^{2}$. We applied this fitting technique to (i) the muon bundle multiplicity distribution (Fig. 7.6), (ii) the average multiplicity as a function of the zenith angle (Fig. $7.7(\mathrm{a})$ ), and (iii) the average multiplicity as a function average intermuon separation (Fig. $7.7(b)$ ). The calculated percentages for $f_{p}$ and $f_{F_{e}}(\%)$ are given in Table 7.2. All three methods give consistent results for $f_{p}$ and $f_{F e}$, indicating that the fraction of protons in the primary cosmic radiation is about three times higher than the fraction of iron nuclei for primary energies represented by our data. 


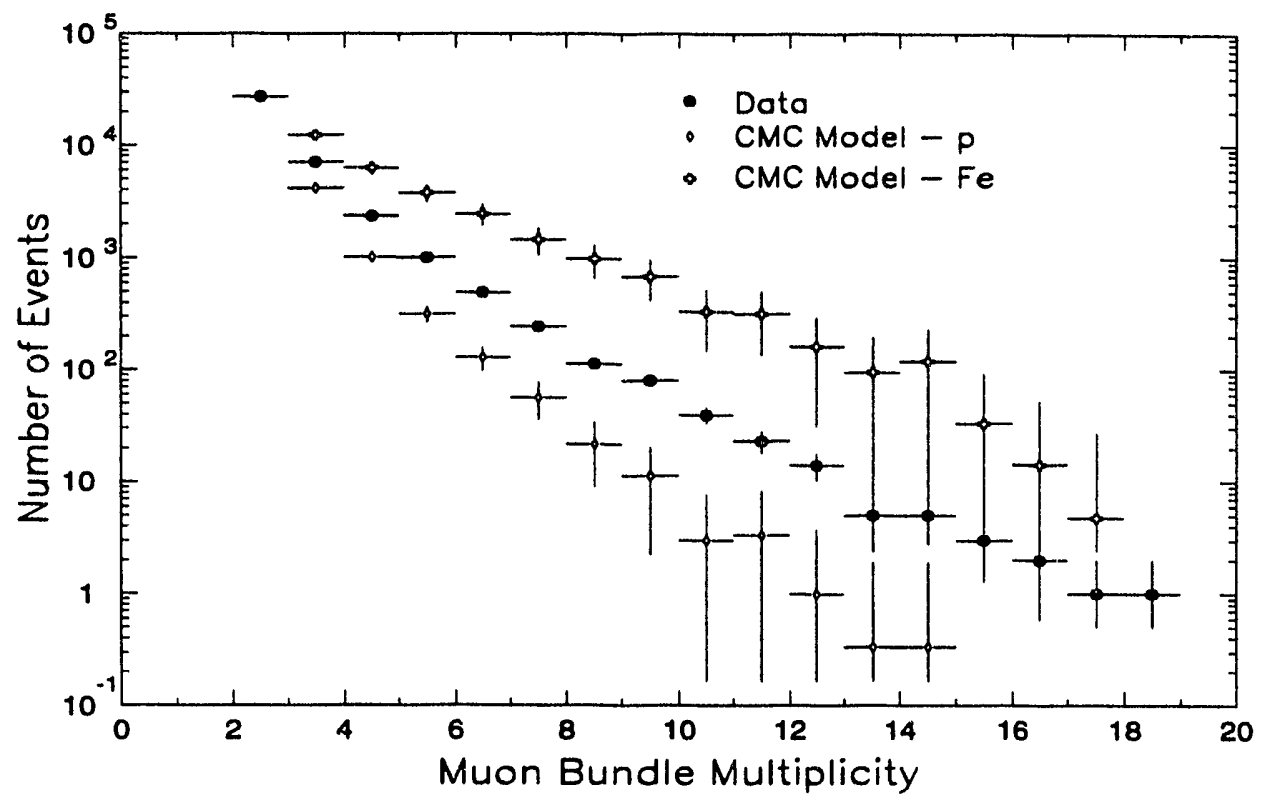

Figure 7.6: Multiplicity distributions for data and for the CMC model with $p$ and Fe primaries only (normalized to the number of two muon events in the data sample).

Table 7.2: Percentages of protons and iron in the primary cosmic rays for rock density of $2.80 \mathrm{~g} \mathrm{~cm}^{-3}$

\begin{tabular}{|l|c|c|}
\hline Method & $f_{\mathrm{p}}(\%)$ & $f_{F_{\mathrm{e}}}(\%)$ \\
\hline Multiplicity & $77 \pm 7$ & $23 \pm 7$ \\
\hline$\left\langle N_{\mu}\right\rangle$ Vs $\cos \theta$ & $76 \pm 2$ & $24 \pm 2$ \\
\hline$\left\langle N_{\mu}\right\rangle$ Vs $\left\langle R_{i j}\right\rangle$ & $72 \pm 2$ & $28 \pm 2$ \\
\hline
\end{tabular}



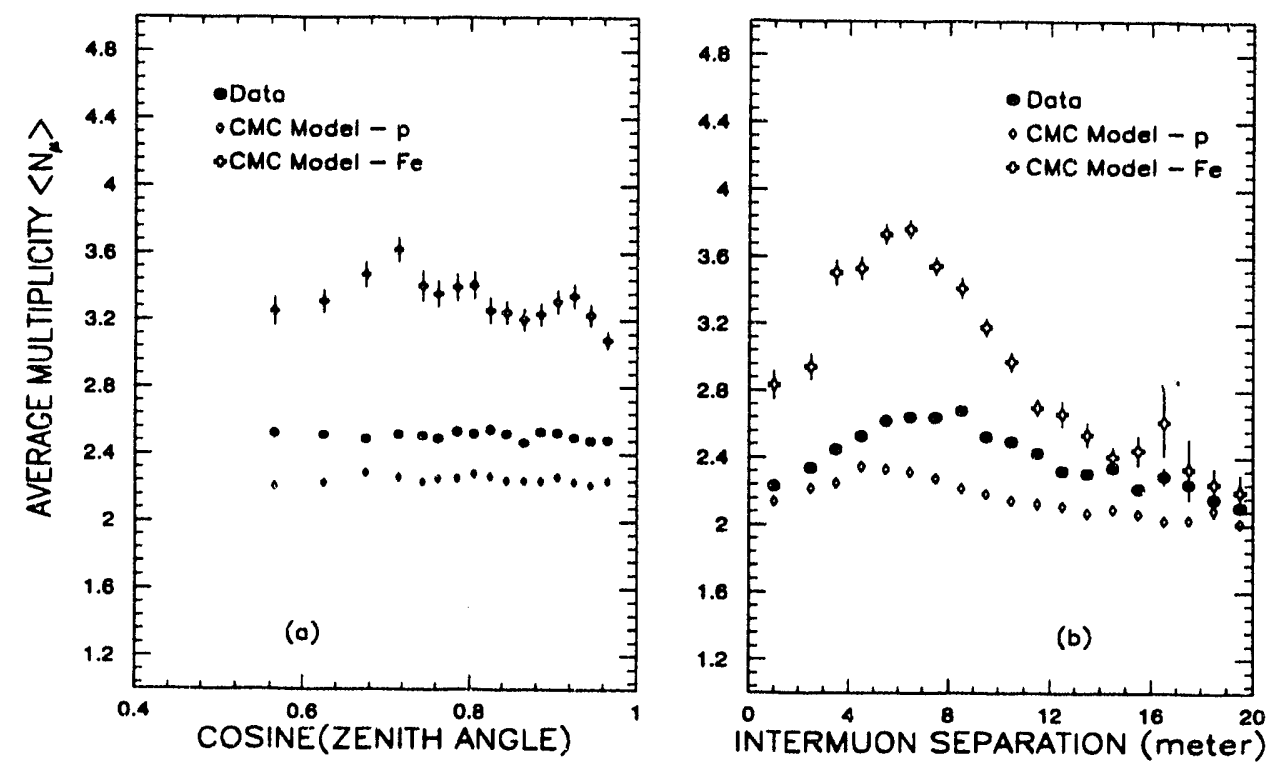

Figure 7.7: (a) Average multiplicity versus zenith angle for data and for CMC model with $p$ and Fe primaries; (b) average multiplicity versus the average intermuon separation for the same model. 


\section{Chapter 8 \\ Discussion and Conclusion}

\subsection{Overview}

The purpose of this Thesis is to collect and to analyze underground multiple muon events recorded by the Soudan 2 experiment, in order to study primary cosmic ray composition within the energy range between $10 \mathrm{TeV}$ and $10^{5} \mathrm{TeV}$. These muon bundle events originate with interactions of primary cosmic rays in the upper atmosphere. By studying muon bundles using deep underground detectors, features of the primary cosmic ray nuclear composition can be inferred.

The analysis approach of this Thesis is a "pioneering effort" in that it is the first time within Soudan 2 that all of the various hit configurations recorded by the cavern-liner veto shield array have been utilized in a unified way, in conjunction with central detector triggering and tracking, for multiple muon study. The main advantage in using the veto shield for muon studies is its large area coverage. The ceiling area is $420 \mathrm{~m}^{2}$; the south, east, and west walls constitutes an additional 740 square meters of instrumented area. Although the veto shield has limited twotrack resolution and various inefficiencies, we are able nevertheless to reconstruct muon bundles with high multiplicity (20 to 25 ) and with large separations between muon pairs (up to 30 meters) because of the shield's large area. The separation between muon pairs is a measure of the transverse momentum of hadrons produced in the interactions of primary cosmic rays in the upper atmosphere. In principle, the lateral distribution contains information about the cosmic ray - air interaction and also contains information about the height of primary interaction points in the 
upper atmosphere.

Since bundle multiplicity and intermuon separation are correlated - within any specific interval of primary cosmic ray energy - with cosmic ray nuclear composition, large area detection is essential for state-of-the art studies with underground detectors. The Soudan shield area compares favorably with the detection areas utilized by other recent experiments. For example, the too areas for the NUSEX [17] and Frejus [14] planar, iron tracking calorimeters were $12 \mathrm{~m}^{2}$ and $100 \mathrm{~m}^{2}$ respectively. The area of the Homestake detector, which was an array of water Cherenkov modules located in the Homestake gold mine, was $113 \mathrm{~m}^{2}$ [58]. The recently published MACRO study [3] of multiple muons was obtained with two currently operating "supermodules", whose top surface area totals $288 \mathrm{~m}^{2}$. When completed, the MACRO detector will have a top area of about $900 \mathrm{~m}^{2}$ for muon tracking. The analysis of this Thesis is also 'pioneering' in that it utilizes data from the shield in relatively early stages of the Soudan experiment. Improvements to the shield array have been made continually since the data of the present analysis were taken. Additionally, there are major upgrades envisaged for the shield system. The work of this Thesis, then, provides a foundational analysis for future multiple muon studies. Future investigations will benefit from the on-going enhancement of the shield's tracking capability, and so should be able to reduce many of the systematic uncertainties delineated by the present work.

A significant near-term enhancement of the veto shield's tracking capability, towards which a portion of this author's research has been dedicated (see Chapter 2, Section 2.5 and Appendix D), will be the deployment of the HPW proportional tube array. By criss-crossing HPW panels with existing shield panels, it will be possible to measure the coordinates of the exit point of a muon track more precisely. It is desirable, over the long term, to deploy panels transversely not only on the ceiling but on every superpanel. These additional panels could remove the trigger holes (north-south and east-west) in the central detector if the enhanced veto shield 
were utilized for triggering.

All gap fillers and the major portion of the north wall are now taking data. Two HPW panels have been collecting data together with the standard shield panels. In the near future, more HPW panels will be operational, hopefully covering both the ceiling and the floor. Studies with another set of proportional chambers (TASSO tubes, acquired from the UK), which will be deployed in the future along the vertical wall sections, were completed last summer [59]. When the enhanced shield system is completely deployed with criss-crossed panels, the shield will then be a stand-alone muon telescope for carrying out cosmic ray studies.

We have examined the properties of observed multiple muons and have compared various distributions of thesc properties with four cosmic ray composition models (Chapter 5 and 6). Muon multiplicity depends on the primary composition. This is clearly seen in Fig. 6.7 which shows the CMC model prediction of muon multiplicity for different primary energies. Iron primaries contribute higher multiplicity events in the energy range between $1000 \mathrm{TeV}$ to $10^{5} \mathrm{TeV}$. Our observed muon multiplicity distribution is well-described by the two light nuclei composition models which are the CMC and the Linsley models. Our bundle multiplicity comparison suggests that the primary cosmic ray composition contains mostly protons with a modest percentage of heavy nuclei within the energy region between $100 \mathrm{TeV}$ and $10^{5} \mathrm{TeV}$.

We have calculated the absolute rate for occurrence of muon bundles at the Soudan 2 site. The muon flux depends upon the rock overburden, and we have considered three different densities for Soudan rock in our Monte Carlo simulations of events, in order to compare to the data. With the rock density $2.80 \mathrm{~g} \mathrm{~cm}^{-3}$, the light composition models, CMC and Linsley, show better agreement with our data. The other two models which represent heavy composition imply wider multiplicity distributions than observed. The same trend has been reported by MACRO [3] and by NUSEX [17].

Although all four models predict intermuon separations similar to that of the 
data sample, our data exhibit a wider separation. Since there is very little difference among the four models in this parameter, there is little discrimination although the Maryland model appears to be slightly favored. We have searched for correlations among bundle multiplicity, zenith angle and intermuon separation. We find the average multiplicity to be almost constant within the zenith angle between $15^{\circ}$ and $60^{\circ}$. The average intermuon separation increases slightly with increasing zenith angle within the angular range given above. A definite increase in intermuon separation with zenith angle has been reported by MACRO [3]. According to our analysis, the average intermuon separation increases with muon bundle multiplicity. Although this trend is described by all four models, the models do not reproduce its overall extent in the data. No winer experiments have reported this correlation to date. However, the Monte Carlo study done by C. Forti et al. (Ref. [37], Fig. 14) indicates that correlations among muon bundle multiplicity, mean perpendicular distance between the muons and the shower core, and height of production are very likely. For $1000 \mathrm{TeV}$ protons at a zerith angle $28^{\circ}$ Ref. [37] finds an increas in mean separation with increasing multiplicity. They indicate that no such correlation are to be expected for heavy primaries.

In order to extract representative numbers for the relative contributions of light versus heavy nuclei in primary cosmic rays around the knee regions of the flux, we assumed the primaries to consist of only protons and of iron nuclei, and utilized fitting of distributions to minimum $\chi^{2}$. We used three different methods to extract representative percentages. All three methods indicate that "protons" are the dominant cosmic ray primary which produces underground muon bundles at Soudan. The light nuclei appear to comprise between 70 to 74 percent of the primary flux. 


\subsection{Discussion}

Muon bundles, which are produced in the interaction of primary cosmic radiation with nitrogen and oxygen nuclei of the upper atmosphere, have been studied by most of the underground experiments. Results pertaining to cosmic ray primaries of $10^{2}$ $\mathrm{TeV}-10^{5} \mathrm{TeV}$ incident energy have been reported by Soudan 1 [42], NUSEX [17], Frejus [14], MACRO [3] and by Soudan 2 [54]. These studies have presented evidence for light composition, mostly protons, in the primary cosmic ray composition around and above the knee region $\left(\approx 3 \times 10^{3} \mathrm{TeV}\right)$. The analysis of this Thesis provides new, additional evidence in support of the occurrence of a light nuclear composition in the knee region of the primary cosmic ray flux.

Direct measurements with balloons and satellite experiments in the upper atmosphere provide good information of primary cosmic ray composition in the energy range between $10 \mathrm{GeV}$ and $100 \mathrm{TeV}$. Although the primary composition in the latter range is predominantly light, there are significant portions of heavy nuclei in this energy range. Results from the JACEE collaboration [12] show that the percentages of $p$, He, $\mathrm{C}-\mathrm{O}, \mathrm{Ne}-\mathrm{S}$, and heavy elements $(\mathrm{Z}>17)$ are $(12 \pm 9) \%$, $(25 \pm 14) \%$, $(26 \pm 12) \%,(15 \pm 8) \%$, and $(21 \pm 10) \%$ around the energy region $4 \times 10^{2} \mathrm{TeV}$ per particle. A report from 'Chicago Egg' [55] satellite experiment claims that the spectrum is almost flat for the Fe group up to $1,00 \mathrm{TeV} / \mathrm{amu}$ (the slope $\gamma=2.55 \pm$ 0.09). A question raised in the recent analysis by a researcher at the Institute for Cosmic Ray Research (ICRR, Japan) [56], is whether or not the proton and iron components, as a function of increasing primary energy, "cross" each other. They claim that the components do not cross for the energies below $100 \mathrm{TeV}$. If all of the direct measurements below $100 \mathrm{TeV}$ are taken together, then it seems that there is a substantial fraction of heavy nuclei in the primary cosmic ray composition in the energy region 0.01 to $100 \mathrm{TeV}$.

For energies above the knee region, the analysis of X. Chi et al. [57] using ground 
array data from Haverah Park (UK), from Sydney (Australia), from Volcano Ranch (USA), and from Yakutsk (former USSR), shows strong evidence for an increasing trend in the heavy nuclei in the energy range between $10^{5} \mathrm{TeV}$ and $10^{7} \mathrm{TeV}$. However, at the highest energy of $3 \times 10^{7} \mathrm{TeV}$, protons again dominate. The Fly's eye experiment, which is another big ground array (USA) has reported a new analysis very recently [12]. They calculate the percentages for nuclear composition in different energy ranges. The percentage of iron in the energy between $3 \times 10^{5}$ and $5 \times 10^{5}$ $\mathrm{TeV}$ is $79 \%$ and it decreases to $56 \%$ around $10^{6} \mathrm{TeV}$ and above, according to their analysis. It should be noted that primary cosmic rays with energy below $10^{7} \mathrm{TeV}$ are widely believed to be of galactic origin. Above this energy, the cosmic ray spectrum flattens and is conjectured to be dominated by protons which may originate from the active galactic nuclei [2].

\subsection{Conclusion}

The knee region in the primary cosmic ray energy spectrum is a very interesting region to study cosmic ray nuclear composition. Our analysis of multiple muon events recorded in Soudan 2 favors the light composition models (the CMC and the Linsley models) which predict mostly protons together with a modest admixture of heavy elements. Around the knee region, the underground experiments are unanimous in favoring a light composition.

According to balloon and satellite experiments, protons and other light nuclei such as He constitute the majority of the incident flux below the knee region, however the amount of heavy primaries such as Fe is significant. Well above the knee region ( $>10^{5} \mathrm{TeV} /$ nucleus), analysis of surface array data indicate that the heavy primaries dominate. At the highest energies $\left(>10^{7} \mathrm{TeV}\right)$, cosmic ray protons are again predominant.

Fig. 8.1 summarizes the reported fractions of light versus heavy nuclei, versus 


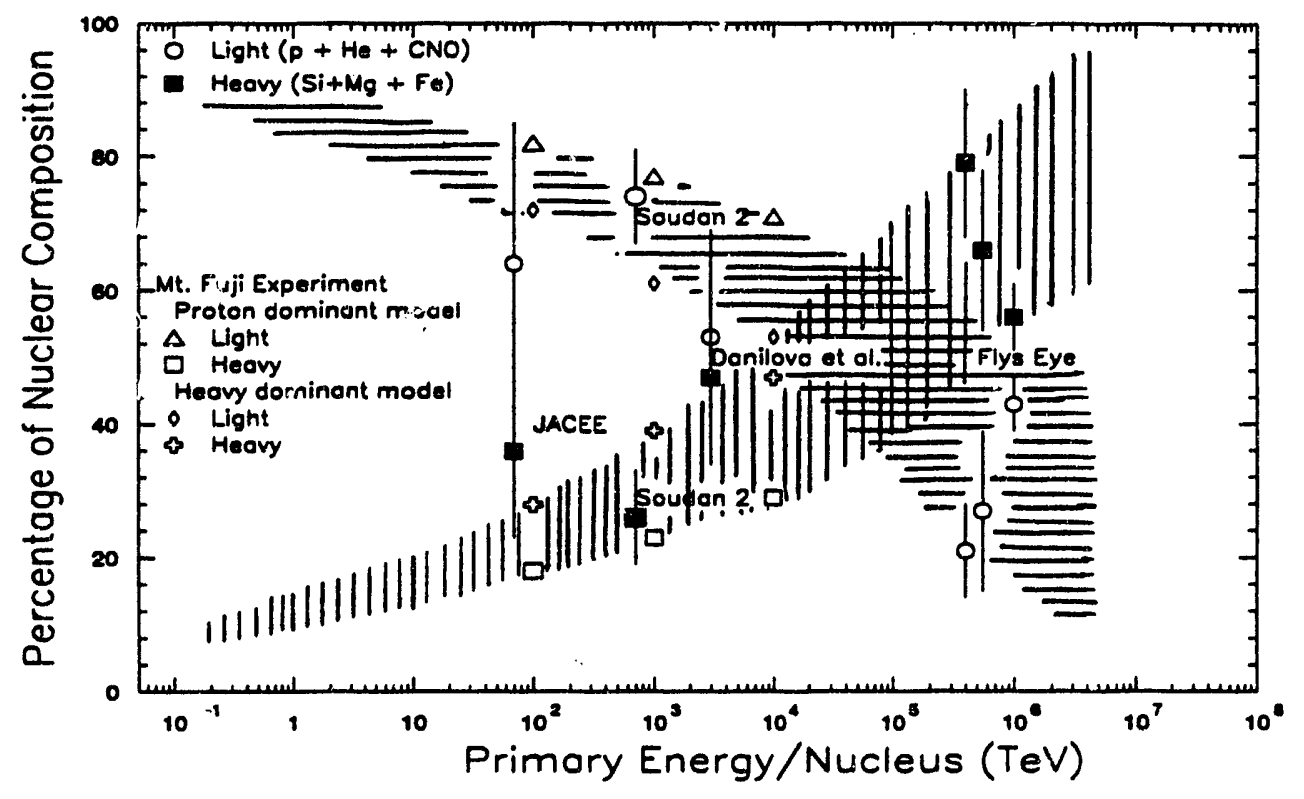

Figure 8.1: Fractional cosmic ray composition; world data

primary energy per nucleus. The shaded areas are drawn by hand according to the information collected from various reports for proton and iron primaries respectively; ihey suggest a dramatic variation of composi.ion with increasing energy scale. In v-w of such extensive variations, it is likely that a variety of mechanisms are involved in cosmic ray acceleration. 


\section{Appendix A}

\section{The Soudan 2 Collaboration}

W. W. M. Allison ${ }^{(3)}$, G. J. Alner ${ }^{(4)}$, I. Ambats ${ }^{(1)}$, D. S. Ayres ${ }^{(1)}$, L. J. Balka(1), G. D. Barr ${ }^{(3),(a)}$, W. L. Barret ${ }^{(8),(1)}$, D. Benjamin ${ }^{(5)}$, P. M. Border ${ }^{(2)}$, C. B. Brooks ${ }^{(3)}$, J. H. Cobb(3), D. J. A. Cockerill( ${ }^{(4)}$, H. Courant ${ }^{(2)}$, J. W . Dawson ${ }^{(1)}$, D. M. Demuth ${ }^{(2)}$, V. W. Edwards ${ }^{(4)}$, B. Ewen ${ }^{(5)}$, T. H. Fields ${ }^{(1)}$, C. Garcia-Garcia( ${ }^{(4),(b)}$, R. H. Giles ${ }^{(3)}$, G. L. Giliar( ${ }^{(3)}$, M. C. Goodman ${ }^{(1)}$, R. N. Gray ${ }^{(2)}$, S. J. Heilig(6),(1), N. Hill(1), J. H. Hoftiezer ${ }^{(1)}$, D. J. Jankowski ${ }^{(1)}$, K. Johns ${ }^{(2),(d)}$, T. Kafka( ${ }^{(5)}$, S. M. S. Kasahara ${ }^{(2)}$, J. Kochocki( ${ }^{(5),(f)}$, W. Leeson ${ }^{(5)}$, P. J. Litchfield( ${ }^{(4)}$, N. P. Longley( ${ }^{(2)}$, F. V. Lopez ${ }^{(1)}$,

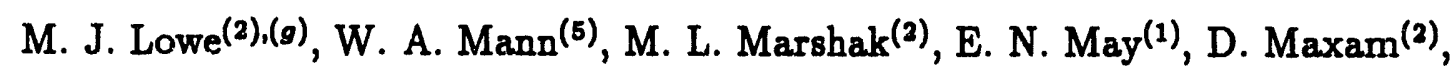
L. McMaster ${ }^{(5)}$, R. H. Milburn ${ }^{(5)}$, W. H. Miller ${ }^{(2)}$, C. P. Minor ${ }^{(2)}$, A. Napier(5), W. P. Oliver ${ }^{(5)}$, G. F. Pearce ${ }^{(4)}$, D. H. Perkins ${ }^{(3)}$, E. A. Peterson ${ }^{(2)}$, L. E. Price ${ }^{(1)}$, D. M. Roback(2), D. B. Rosen ${ }^{(2),(h)}$, K. Ruddick ${ }^{(2)}$, B. Saitta ${ }^{(5),(i)}$, D. J. Schmid ${ }^{(2)}$, J. Schlereth ${ }^{(1)}$, J. Schneps ${ }^{(5)}$, P. D. Shield ${ }^{(3)}$, M. A. Shupe ${ }^{(2),(d)}$, N. Sundaralingam ${ }^{(5)}$, M. A. Thomson ${ }^{(3),(k)}$, J. L. Thron ${ }^{(1)}$, L. M. Tupper ${ }^{(3),(e)}$, G. Villaume ${ }^{(2)}$, S. J. Werkema ${ }^{(2),(j)}$, N. West ${ }^{(3)}$ and C. A. Woods ${ }^{(4),(a)}$ 
(1) Argonne National Laboratory, Argonne, IL 60499, USA

(2) University of Minnesota, Minneapolis, MN 55455, USA

(3) University of Oxford, Oxford OX19RH, UK

(4) Rutherford Appleton Laboratory, Chilton, Didcot, Oxfordshire, OX110QX, UK

(5) Tufts University, Medford, MA 02155, USA

(6) Greinnel College, Grinnel, IA 50112, USA

(7) Carleton College, Northfield, MN 55057, USA

(8) Western Washington University, Bellingham, WA 98225, USA

(a) Now at CERN, CH-1211, Geneva 23, Switzerland

(b) Now at IFIC, E-46100 Burjassot, Valencia, Spain

(c) Now at LeCroy Corporation, Chestnut Ridge, NY 10977, USA

(d) Now at University of Arizona, Physics Department, Tucson, AZ 85721, USA

(e) Now at University of Oxford, Department of Physiology, Oxford, UK

(f) Now at Virginia Polytechnic Institute, Blacksburg, VA 24061, USA

(g) Now at Rice University, Houston, TX 77251, USA

(h) Now at Boston University, Boston, MA 02215, USA

(i) Now at Univ. di Ferrara, Dipartimento di Fisica, 4100 Ferrara, Italy

(j) Now at FNAL, Batavia, II 60510, USA

(k) Now at Department of Physics and Astronomy, University College London, London WC1E 6BT, UK 


\section{Appendix B}

\section{Data Summary Tape Format}

The format below was used to summarize multiple muon events on an eventby-event basis. The data summary tape (DST) is a linear vector written in binary format. For each multiple muon event, the DST is organized into six different blocks, namely the Header Block, the Hitbox Block, the Adjc Block, the Single Hit Block, the Splat Block, and the Candidate Muon Trajectory Block. The format for each block is as follows:

1. HEADER BLOCK

Number of words in entire event record, counting this word.

Number of words in the header block,including word \# 1 and this word. (The value of this word is 13 ).

Exabyte number where the DST binary files are saved. (The Value of this word is 2. The Tape number is DST002).

Run number.

Event number.

Date.

Time-(HHMMSSCC). This word is positive if the time is from WWVB, negative if it is taken from the VAX.

Time-(Millisecond $\times 10000)$.

Trigger Word (Packed).

Event Type (1 or 2 or 3 ). 
Note: Event Type 1 = Candidate partially contained events.

Event Type 2 = Candidate horizontal muon events.

Event Type 3 = Candidate multiple muon events.

DST_ V2.FOR program version number (2).

The date when the DST was created.

Blank word.

\section{HITBOX BLOCK}

Number of words in the Hitbox Block, including this word.

Number of tracks passing through the Central Detector.

$T_{0}$ from the central detector.

The Following coordinates are for the Shield Box:

Hitbox coordinates (6).

Direction Cosine $(\alpha, \beta, \gamma)$.

The following coordinates are for the Detector Box:

Hitbox coordinates (6).

Calculated Time Slot (TS) using the central detector $T_{0}$.

Slope (a) in line parameterisation $z=a x+b$ for Anode vs Time.

Intercept (b) for the above line.

Slope (c) in line parameterisation $z=c y+d$ for Cathode vs Time.

Intercept (d) for the above line.

$T_{0}$ for Anode time.

$T_{0}$ for Cathode time.

Error on slope (a) in line parameterisation $z=a x+b$ (Anode vs Time).

Error on constant (b) in line parameterisation $z=a x+b$.

Error on slope (c) in line parameterisation $z=c y+d$ (For Cathode vs Time).

Error on constant (d) in line parameterisation $z=c y+d$.

Error on $T_{0}$ (Anode Vs Time). 
Error on $T_{0}$ (For Cathode Vs Time).

Packed word for face number:

Face $(1) \times 1000+$ Face $(2)$

For second, third, ..... tracks the format is repeated, starting

from "hitbox coordinate (for the shield box)" above.

3. ADJC BLOCK( Summarizes the Adjacent Group hits in the veto shield)

Number of words in the ADJC Block, including this word.

Number of Groups of adjacent hits.

For the first group:

Time span (TSPAN).

Number of hits in this group.

$\mathrm{XYZ}$ - coordinates for all four corners (12).

Leading edge TS, Panel, First Tube, INOUT packed word.

$$
(\mathrm{TS} \times 10000000+\mathrm{Panel} \times 10000+\text { Tube } \times 10+1 \text { or } 0)
$$

Trailing edge TS, Panel, Last Tube, INOUT packed word.

For the second, third ....... groups, the format repeats beginning with

"Time span" above.

\section{SINGLE HIT BLOCK}

Number of words in this block, including this word.

Number of single hits.

Packed word for TS, Panel, Tube, In-Out for this hit.

TS $\times 10000000+$ PANEL $\times 10000+$ TUBE $\times 10+$ INO

XYZ-coordinates for both end of the tube (6).

This sequence repeats for the other single hits, starting from "packed word" above. 


\section{SPLAT BLOCK}

Total number of words in this block, including this word.

Total number of splats.

Index number of the first splat.

Index number (order in ADJC block) of first adjacent group in this splat.

If there is more than one group in the splat, the index number of the splat is same as the previous one, but group index varies.

(eg: $\{(1,1)\},\{(2,2),(2,3)\}, \ldots . . e t c)$

Repeats, starting from the index number, for second, third,..... splats.

6. CANDIDATE MUON TRAJECTORY BLOCK.

\subsection{Class \# 1 Candidate Muons.}

Total number of words in this block, including this word.

Number of muons within main detector and with high and or low hits in the shield.

For first muon in this category:

Index number for this muon from the Hitbox Block.

Packed index word for associated high hits, adjacent group, or single for this muon:

For an associated adjacent group -

Packed word $=1000 \times$ Index number of the group +1

For single hit -

Packed word $=1000 \times$ Index number of single +2

This word is zero if the candidate track has no high hits and traverses a panel.

Word is 3 if the candidate track has no high hits and traverses a gap.

Coordinate for the high hit (3). 
Packed index word for associated low hits, adjacent groups, or single hits for this muon.

(Packed word has format identical to the high hits word.)

Coordinate for the low hit (3).

Errors on Coordinates for both the high and low hit (6).

The format repeats for second, third, .... muon reconstructed with the main detector.

\subsection{Class \# 2 Candidate Muons}

Total number of words in this sub block, including this word.

Total number of class \# 2 muons.

For the first muon in this category:

Packed index word for associated high hits, adjacent group, or single hits for this muon.

For group and non-parallel combinations:

Packed word $=10000 \times$ Index number of groups +101

For single and non parallel combinations:

Packed word $=10000 \times$ Index number of single +201

For group and parallel combinations:

Packed word $=10000 \times$ Index number of groups +100

For single and parallel combinations:

Packed word $=10000 \times$ Index number of single +200

Coordinates for high hit (3).

Packed index word for associated low hits, adjacent groups, or single hits for this muon.

Packed word is the same as for the high hits word.

Coordinate for low hit (3).

Errors on Coordinates (6).

This format repeats for second, third, .... muons not associated with main detector. 
6.3 Class \# 3 Candidate Muons:

Total number of words in this sub-block, including this word.

Number of class \# 3 muons.

For the first muon in this category:

Coordinates for both ends of a track anywhere in the shield.

The above format repeats for second, third,... muons in this class.

6.4 Class \# 4 candidate muons.

Total number of words in this sub block, including this word.

Number of isolated splats

Total number of groups in all isolated splats.

Splat Identification number.

Index number of the first adjacent group within the first splat.

Coordinates for both ends of the group (6).

Leading edge TS, Panel, First Tube, INOUT packed word.

$($ TS $\times 10000000+$ Panel $\times 10000+$ Tube $\times 10+1$ or 0$)$

Trailing edge TS, Panel, Last Tube,INOUT packed word.

This format repeats starting from splat identification number for second, third,...

group within the same splat, if there is more than one group.

Otherwise, the same format repeats for second, third, .... splats.

6.5 Class \# 5 Candidate Muons:

Total number of words in this sub block, including this word.

Number of isolated singles

Coordinates for first single (6).

This format repeats for the second, third,... single. 
6.6 Class \# 6 Candidate Muons:

Total number of words in this block, including this word.

Total number of splats on the ceiling.

Earliest time for the first splat.

Latest time for the first splat.

The last two words repeat for the remaining splats.

7. MUON BUNDLE BLOCK

Total number of words in this block, including this word.

Total number of CLASS \# 1 and CLASS \# 2 muons.

Average direction cosine for a muon bundle.

Angles between $\mathrm{X}, \mathrm{Y}$ and $\mathrm{Z}$ - Axes and the Muon $(\alpha, \beta$ and $\gamma)$.

Tangent of projected track on $\mathrm{Y}-\mathrm{Z}$ plane.

Tangent of projected track on $Y$-X plane.

Error values on $\alpha, \beta$ and $\gamma$. 


\section{Appendix $\mathbf{C}$ \\ Error Calculation}

Errors for direction cosines and for coordinates of intersection points of a muon track with shield surfaces are calculated and propagated using the errors assigned to the slope and intercepts for a given track by the SEARCH processor. For a candidate muon track in the central detector, two projected views $\mathrm{Z}-\mathrm{X}$ and $\mathrm{Z}-\mathrm{Y}$ planes are reconstructed by SEARCH. The straight-line trajectories in these views satisfy the relations

$$
z=a x+b
$$

and

$$
z=c y+d,
$$

where $a$ and $b$ are the slope and intercept on Z-X plane, and $c$ and $d$ are the slope and intercept on Z-Y plane. Errors on the quantities $a, b, c$, and $d$ are designated by $\Delta a, \Delta b, \Delta c$, and $\Delta d$. Consider a candidate muon track in the central detector which also gives shield hits on a ceiling panel and on a floor panel. Coordinates for the intersection points of this track with the ceiling and floor are designated $\left\{x_{1}\right.$, $\left.y_{1}, z_{1}{ }^{\prime}\right\}$ and $\left\{x_{2}, y_{2}, z_{2}\right\}$ respectively. The errors on $x$ and $z$ coordinates for either intersection are given by the formulas:

$$
\left.\Delta z=\left[\left(c \Delta y_{1}\right)^{2}+\left(y_{1} \Delta c\right)^{2}+(\Delta d)^{2}\right)\right]^{\frac{1}{2}}
$$

and

$$
\left.\Delta x=\left[\left(\left(c y_{1}+d-b\right)\left(\frac{\Delta a}{a}\right)\right)^{2}+\left(\frac{\Delta b}{a}\right)^{2}+\left(\frac{y_{1} \Delta c}{a}\right)^{2}+\left(\frac{c \Delta y_{1}}{a}\right)^{2}\right)\right]^{\frac{1}{2}}
$$


Errors for coordinat es of tracks which intersect other veto shield surfaces are calculated similarly. Nominal errors of $\pm 2.0 \mathrm{~cm}$ are assigned for transverse coordinates $x$ and $z$, associated with east/west walls and with the south wall respectively, and on elevation coordinates $y_{1}$ and $y_{2}$.

The length of the track between the above two coordinates is designated $L$ and its error is $\Delta L$. Direction cosines $\alpha, \beta$ and $\gamma$ are given by

$$
\begin{aligned}
& \alpha=\frac{x_{1}-x_{2}}{L} \\
& \beta=\frac{y_{1}-y_{2}}{L} \\
& \gamma=\frac{z_{1}-z_{2}}{L}
\end{aligned}
$$

with errors designated by $\Delta \alpha, \Delta \beta$ and $\Delta \gamma$ respectively. The direction cosine errors can be calculated by using the Eqs. (C.5), (C.6), and (C.7):

$$
\begin{aligned}
& \left.\Delta \alpha=\alpha\left(\left(\Delta x_{1}+\Delta x_{2}\right)^{2}+(\Delta L / L)^{2}\right)\right)^{\frac{1}{2}} \\
& \left.\Delta \beta=\beta\left(\left(\Delta y_{1}+\Delta y_{2}\right)^{2}+(\Delta L / L)^{2}\right)\right)^{\frac{1}{2}} \\
& \left.\Delta \gamma=\gamma\left(\left(\Delta z_{1}+\Delta z_{2}\right)^{2}+(\Delta L / L)^{2}\right)\right)^{\frac{1}{2}}
\end{aligned}
$$

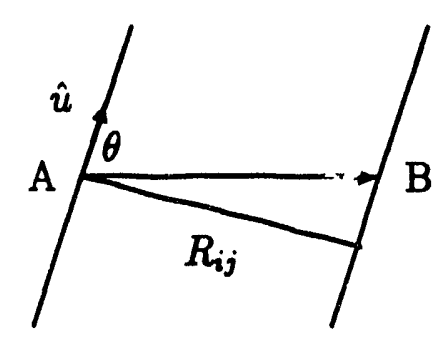

Figure C.1: Distance of perpendicular separation between two parallel muon tracks

The perpendicular distance $R_{i j}$ between pairs in a muon bundle is calculated using the coordinates of entering points and the direction cosines of these tracks. As 
indicated in Fig. C.1, we can calculate the separation in the horizontal plane:

$$
|\overrightarrow{A B}|=\left(\left(x_{i}-x_{j}\right)^{2}+\left(y_{i}-y_{j}\right)^{2}+\left(z_{i}+z_{j}\right)^{2}\right)^{1 / 2}
$$

Then the perpendicular distance $R_{i j}$ is given by

$$
R_{i j}=|\overrightarrow{A B}| \sin \theta
$$

Here, $\theta$ is the angle between the bundle direction $\hat{u}$ and the line joining the two entering points of the tracks, as shown in Fig. C.1. The angle $\theta$ is calculated using

$$
\cos \theta=\frac{\hat{u} \cdot \overrightarrow{A B}}{|\hat{u}||\overrightarrow{A B}|}
$$

The error on $R_{i j}$ is

$$
\Delta R_{i j}=R_{i j}\left[\left(\frac{\Delta|\overrightarrow{A B}|}{|\overrightarrow{A B}|}\right)^{2}+\left(\frac{\Delta(\sin \theta)}{\sin \theta}\right)^{2}\right]^{1 / 2},
$$

where the component errors $\Delta|\overrightarrow{A B}|$ and $\Delta(\sin \theta)$ can be calculated using equations (C.11), (C.12) and (C.13). 


\section{Appendix D \\ Translator Circuits for the HPW Electronics}

\section{D.1 HPW Chambers}

The Harvard-Purdue-Wisconsin (HPW) proportional chambers, acquired in September 1987 from the HPW proton decay experiment, are constructed from extruded aluminum tubes of $5 \times 14 \mathrm{~cm}^{2}$ rectangular cross-section, with lengths of 6.4 and 6.7 meters. A sense wire is strung down the center of each half of the rectangular cross-section tube. Two field-shaping wires, strung at the center of the $14 \mathrm{~cm}$ span, complete the formation of two proportional tube cells within each chamber as shown in Fig. D.1.

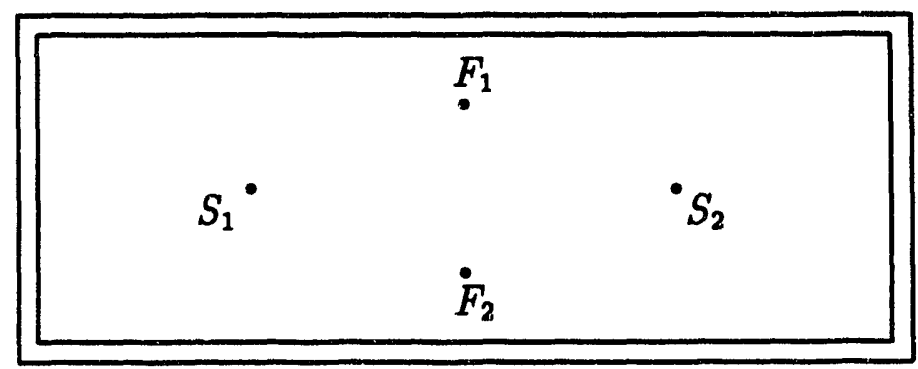

Figure D.1: HPW chamber cross-section

In the Figure,

$S_{1}$, and $S_{2}$ are sense wires, and

$F_{1}$ and $F_{2}$ are field-shaping wires.

The Tufts manifolds and the HPW chambers are to be deployed in a criss-cross configuration to provide both $\mathrm{x}$ and $\mathrm{z}$ coordinates for muons traversing the veto shield ceiling and floor'surfaces. 


\section{D.2 Translator Circuits and Conversion Boards}

The original HPW $r^{\prime}$ cuit boards were designed to receive and to amplify two signals. The sense wires $S_{1}$ and $S_{i}$ are connected together at the end of the chamber opposite to the preamplifier board. When a charged particle traverses the chamber, positive analog signals due to the ionization of the gas are detected by both preamplifiers with the intention of using current division to locate the hit. For our purposes, one of the preamplifiers has been disconnected and the particle crossing is registered digitally using the other channel. The Tufts shield electronics requires digital signals as input to the shield-standard digital read-out modules. Consequently it was necessary to design a translator circuit to change positive analog signals to digital signals.

A schematic diagram of a translator circuit to service one channel is given in Fig. D.2. The circuit contains three integrated electronic chips $U_{1}, U_{2}$ and $U_{3}$. The voltare regulator $U_{\Perp}$, provides a reference voltage which is required by the comparator $v_{1}$. The output voltage $v_{\text {out }}$ of $U_{1}$ drops from its high value to zero volts when the input voltage $\left(v_{\text {in }}\right)$ reaches $30 \mathrm{mV}$. The $v_{\text {out }}$ siays zero until $v_{\text {in }}$ drops to $20 \mathrm{mV}$. This output signal is sent throngh the multivibrator $\left(U_{2}\right)$ and the line driver $\left(U_{3}\right)$ to yield a positive and negative, $1.2 \mu \mathrm{s}$ width digital signal. This latter part of the electronics is similar to the Tufts preamplifier circuit for the Tufts-manufactured veto shield modules. An entire board has sixteen channels to service $16 \mathrm{HPW}$ tubes.

Two prototype boards werc made at Tufts and tested. The final layout of a conversion board is shown in Fig. D.3. A commercial vendor for our circuit board design was found (Cadd Management, Inc. of Torrance, California), and by May 1990 we acquired 45 printed-circuit conversion boards. The boards were stuffed and tested by Tufts technicians, and then shipped to the mine. 


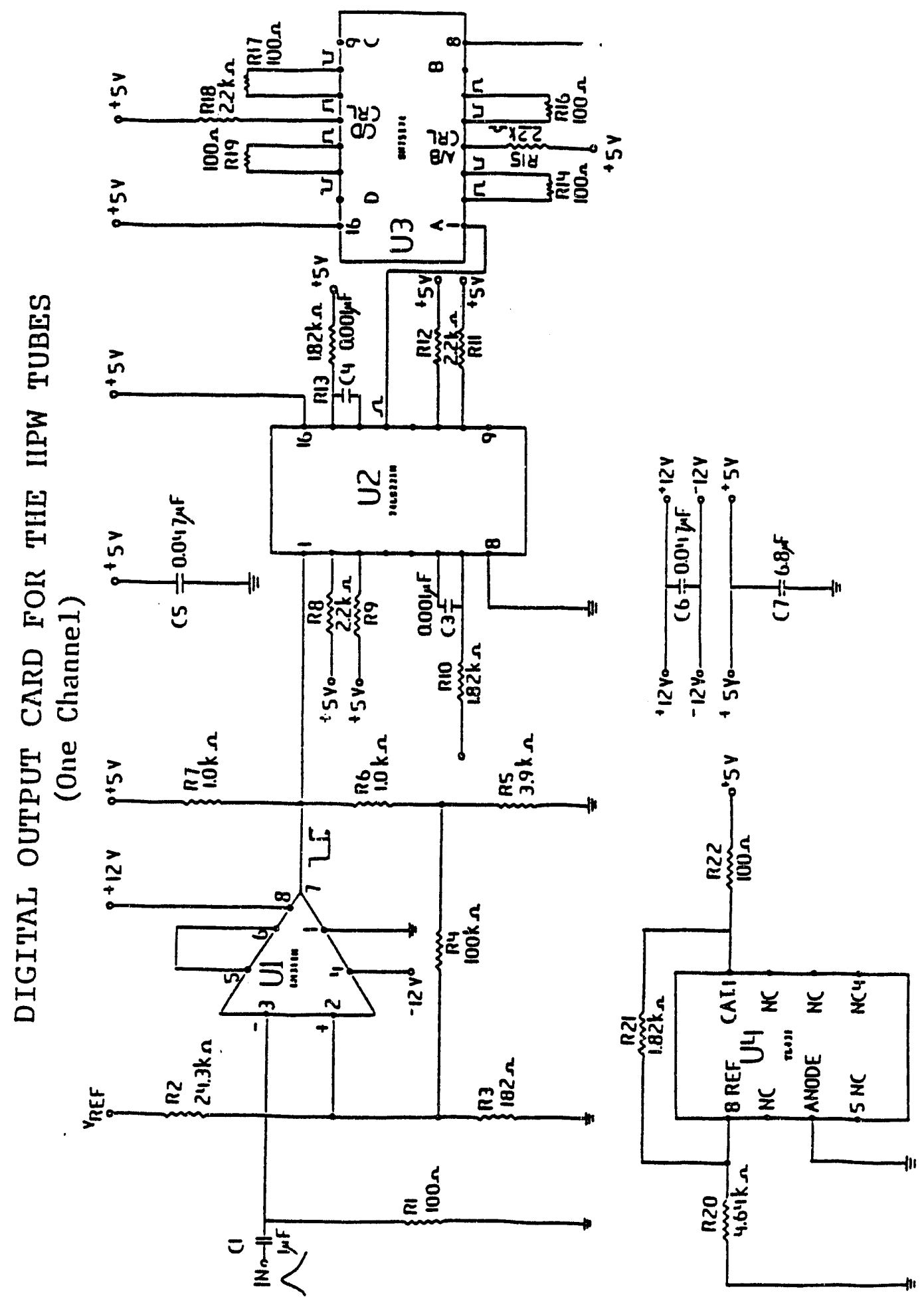

Figure D.2: Schematic diagram for one channel. 

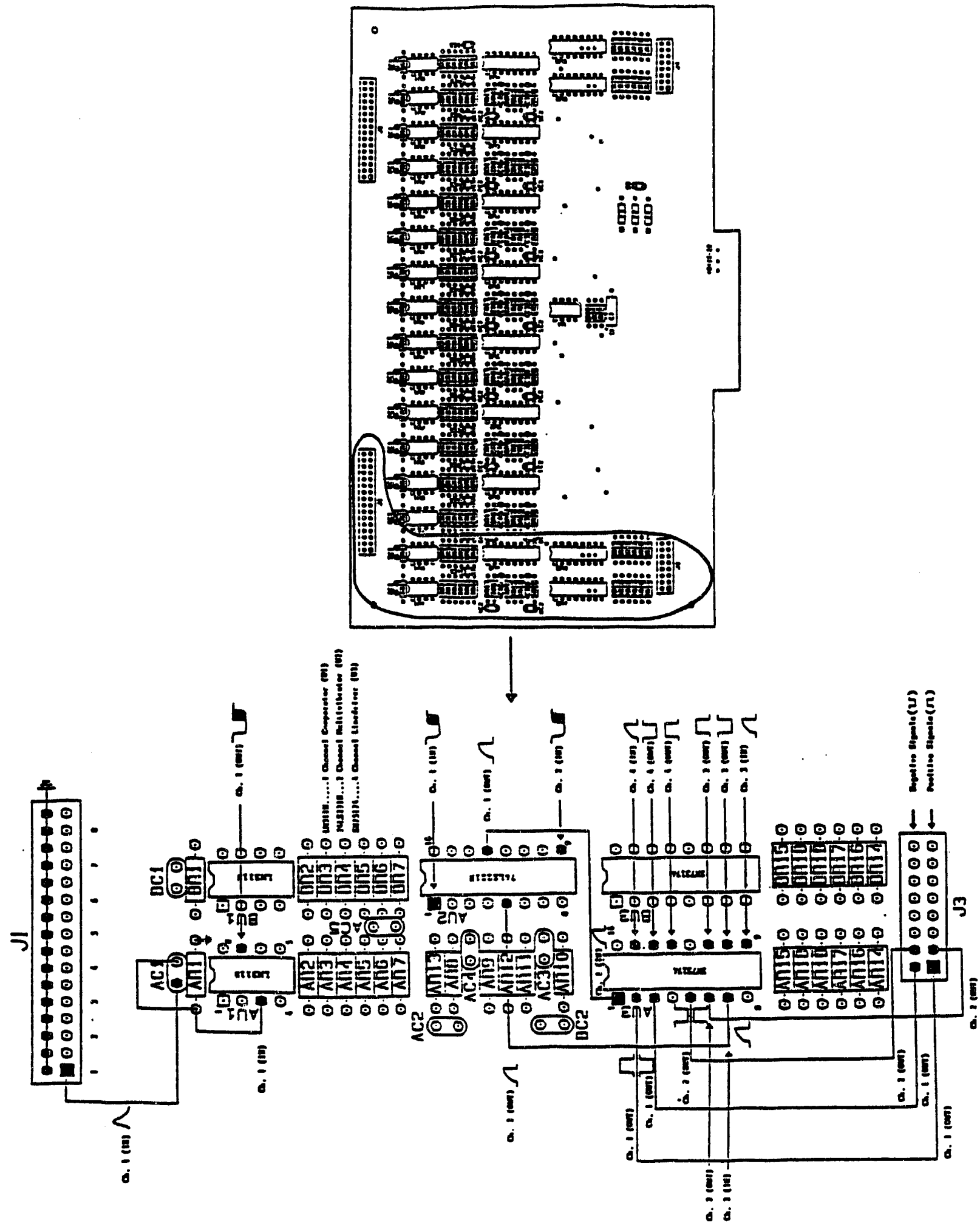

Figure D.3: Board layout for one channel in detail and for entire board. 


\section{References}

[1] Pierre Sokolsky, Introduction to Ultrahigh Energy Cosmic Ray Physics, Addison-Wesley Publishing Company, 1989.

[2] R. J. Protheroe and A. P. Szabo, Phys. Rev. Lett. 69, 2885 (1992).

[3] S. Ahlen et al., Phys. Rev. D 46, 895 (1992).

[4] R. M. Baltrusaitis et al., Nucl. Instrum. Methods A 264, 87 (1988).

[5] M. S. Longair, High Energy Astrophysics, Cambridge University Press, 1981.

[6] M. A. Pomerantz, Cosmic Rays, Van Nostrand Reinhold Co. (1971).

[7] Thomas K. Gaisser, Cosmic Rays and Particle Physics, Cambridge University Press, 1990.

[8] A. W. Wolfendale, Rep. Proz. Phys. 47, 665 (1984).

[9] P. D. B. Collins, A. D. Martin, E. J. Squires, Particle Physics and Cosmology, John Wiley \& Sons, 1989.

[10] W. S. C. Williams, Nuclear and Particle Physics, Oxford Science Publication, 1991.

[11] T. H. Burnett et al., Phys. Rev. Lett. 51, 1010 (1983).

[12] T. K. Gaisser et al., Phys. Rev. D 47, 1919 (1993).

[13] M. A. Thomson et al., Phys. Lett. B 269, 220 (1991).

[14] Ch. Berger et al., Phys. Rev. D 40, 2163 (1989).

[15] Urmimala Das Gupta, Ph.D. Thesis, University of Minnesota, 1989.

[16] H. Adarkar et al., Phys. Lett. B 267, 138 (1991).

[17] M. Aglietta et al., Nucl. Phys. B (Proc. Suppl) 14B, 193 (1990).

[18] G. Battistoni, et al., Phys. Lett. B 133, 454 (1983).

[19] Steven John Werkema, Ph.D. Thesis, University of Minnesota, 1989.

[20] David John Schmid, Ph.D. Thesis, University of Minnesota, 1992. 
[21] Donald Michael Roback, Ph.D. Thesis, University of Minnesota, 1992.

[22] D. J. A. Cockerill et al., to be submitted to Nucl. Instrum. Methods (1993).

[23] W. P. Oliver et al., Nucl. Instrum. Methods A 276, 371 (1989).

[24] J. A. Kochocki, PhD Thesis, Tufts University, 1989.

[25] W. P. Oliver, "Veto shield readout module", PDK-289, September 1986.

[26] W. P. Oliver, Tomas Kafka, "Summary of the veto shield readout", PDK-397 May 1989.

[27] N. Sundaralingam, W. A. Mann, "Expectations for multiple muon events on Soudan's level 27", PDK-415, November 1989.

[28] L. McMaster, W. A. Mann, "Inventory for the HPW acquisition", PDK-348, November 1987.

[29] B. Ewen et al., "The HPW enhancement of the active shield", PDK-525, September 1992.

[30] M. A. Thomson, "The edge trigger and muon astroromy", PDK-434, April 1990.

[31] J. L. Thron et al., Phys. Rev. D 46, 4846 (1992).

[32] G. F. Pearcce, "The NDK Bank Book - A reference manual for Soudan BOS banks", PDK-369, May 1988 (Revised in 1992).

[33] W. P. Oliver, Private Communication.

[34] J. Cobb, P. Litchfield, N. West, "Drift velocity determination in the off-line software", PDK-546, January 1993.

[35] M. A. Thomson, "Muon astronomy at Soudan 2", PDK-407, June 1989

[36] Nat Longley, Private communication.

[37] C. Forti et al., Phys. Rev. D 42, 3668 (1990).

[38] Carl E. Fichtel and John Linsley, Astrophys. J. 300, 474 (1986).

[39] J. Kempa and J. Wdowczyk, J. Physics G: Nucl. Phys. 9, 1271 (1983).

[40] J. A. Goodman et al., Phys. Rev. D 26, 1043 (1982).

[41] J. R. Ren et al., Phys. Rev. D 38, 1404 (1988).

[42] U. Das Gupta et al., Phys. Rev. D 45, 1459 (1992).

[43] K. Ruddick, "Some notes on underground muons", PDK-435, May 1990. 
[44] Review of Particle Properties, Phys. Rev. D 45, III. 14 (1992).

[45] J. Engel et al., Phys. Rev. D 46, 5013 (1992).

[46] E. Chatelet et al., J. Phys. G: Nucl. Part. Phys. 17, 1427 (1991).

[47] G. Bologna et al., Nuovo. Cimento. 8C, 76 (1985).

[48] G. Bologna et al., Nucl. Instrum. Methods A 234, 581 (1985).

[49] K. Kobayakawa, Nuovo. Cimento. XLVII B, (1967).

[50] Martin L. Perl, High Energy Hadron Physics, John Wiley \& Sons, 1974.

[51] T. K. Gaisser and Todor Stanev, Proceedings of 1982 Summer Workshop on Proton Decay Experiments, ANL-HEP-PR-82-84 333 (1982).

[52] S. Ahlen et al., Phys. Rev. D 46, 4836 (1992).

[53] J. W. Elbert, T. K. Gaisser and Todor Stanev, Phys. Rev. D 27, 1448 (1983).

[54] Nat Longley, "Ultra high energy cosmic ray composition from eas/underground muon studies at Soudan 2" PDK-541, December 1992.

[55] John M. Grunsfeld et al., Astrophys. J. 327, L31 (1988).

[56] Masakatsu Ichimura et al., ICRR-Report-287-92-25 (1992).

[57] X. Chi et al., J. Phys. G: Nucl. Part. Phys. 18, 539 (1992).

[58] M. L. Cherry et al., Phys. Rev. D 27, 1444 (1983).

[59] K. Karr et al., "User's Guide to the TASSO array", PDK-524, September 1992.

[60] T. V. Danilova, A. D. Erlykin and J. Procureur, J. Phys. G: Nucl. Part. Phys. 19, 429 (1993). 

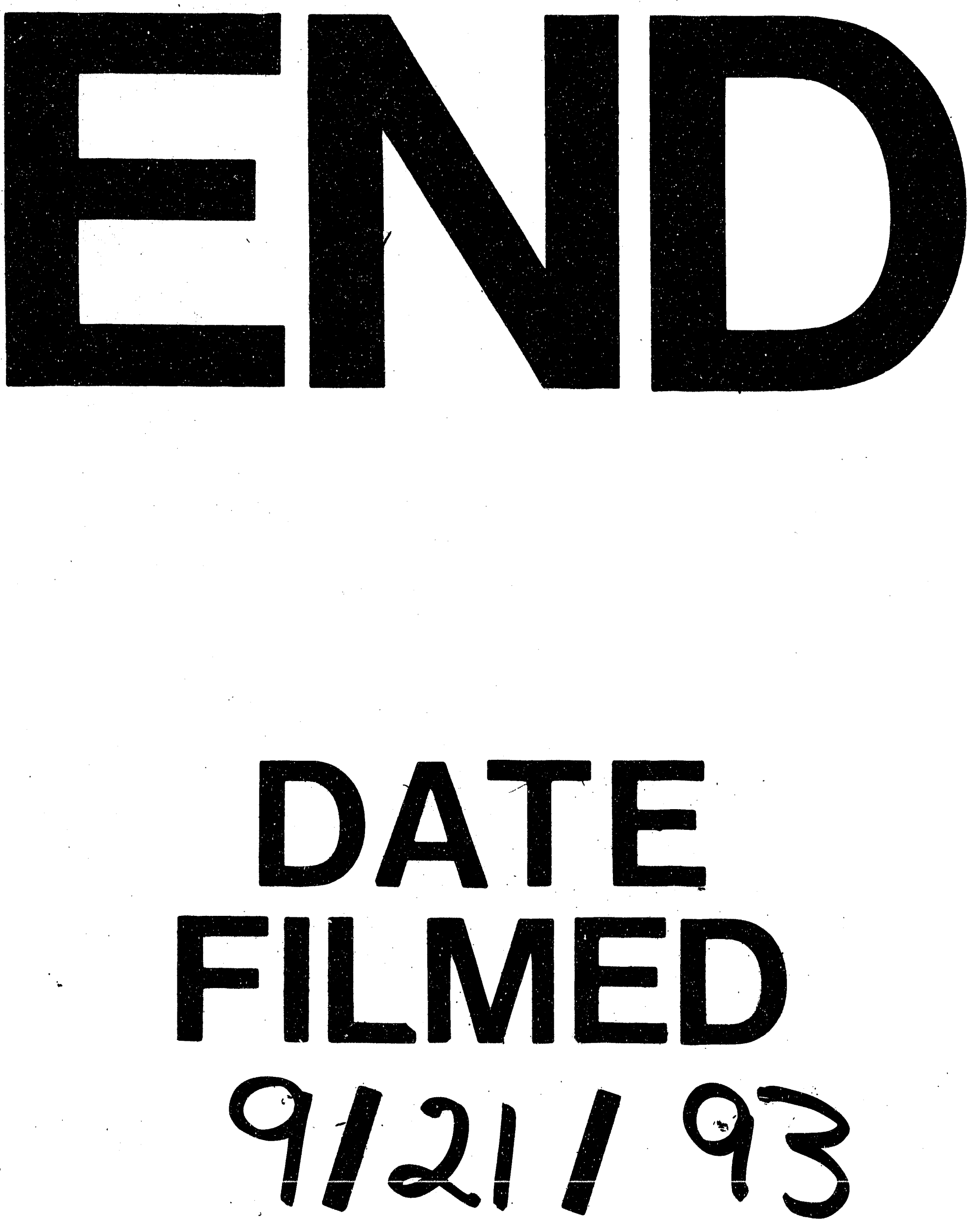

1 
